\title{
Complex Structure and Dynamics of the Heart
}

\author{
Dissertation \\ for the award of the degree \\ "Doctor rerum naturalium" \\ Division of Mathematics and Natural Sciences \\ of the Georg-August-Universität Göttingen
}

submitted by

Philip Bittihn

from Bielefeld

Göttingen, 2013 
Members of the Thesis Committee:

Honorarprofessor Dr. Stefan Luther (First Reviewer)

Biomedical Physics Group

Max Planck Institute for Dynamics and Self-Organization, Göttingen, Germany

apl. Professor Dr. Ulrich Parlitz

Biomedical Physics Group

Max Planck Institute for Dynamics and Self-Organization, Göttingen, Germany

Professor Dr. Eberhard Bodenschatz

Laboratory for Fluid Dynamics, Pattern Formation and Biocomplexity

Max Planck Institute for Dynamics and Self-Organization, Göttingen, Germany

Further members of the examination board:

Professor Dr. Jörg Enderlein (Second Reviewer)

Third Institute of Physics - Biophysics

Georg-August University, Göttingen, Germany

Associate Professor Flavio H. Fenton, PhD

School of Physics

Georgia Institute of Technology, Atlanta, Georgia, USA

Professor Dr. Gerd Hasenfuß

Department of Cardiology and Pneumology

University Medical Center, Göttingen, Germany

Date of the oral examination: June 10, 2013 
I hereby declare that this thesis has been written independently and with no other sources and aids than quoted.

Philip Bittihn 
"If you try and take a cat apart to see how it works, the first thing you have on your hands is a nonworking cat. Life is a level of complexity that almost lies outside our vision; [...]"

Douglas Adams (1952-2001)

The Salmon of Doubt: Hitchhiking the Galaxy One Last Time 


\section{Contents}

\begin{tabular}{llr}
\hline & Introduction & 11
\end{tabular}

1.1 Anatomy of the heart . . . . . . . . . . . . . . . . . . 14

$1.2 \quad$ Physiology of the heart . . . . . . . . . . . . . . . . . . . . 15

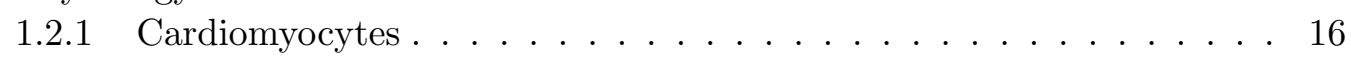

$1.2 .2 \quad$ Cell-to-cell coupling . . . . . . . . . . . . . . . . . . . . . 18

1.3 Structural Heterogeneity . . . . . . . . . . . . . . . . . . . . . . . . 19

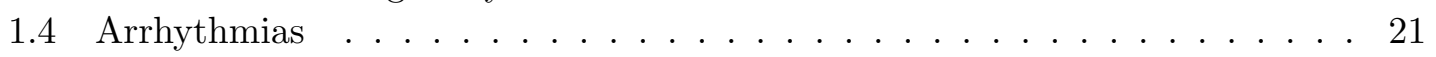

1.5 Antiarrhythmic therapies . . . . . . . . . . . . . . . . . 22

1.6 Complexity in structure and dynamics . . . . . . . . . . . . . . . 23

1.7 This thesis . . . . . . . . . . . . . . . . . . . . . . . 23

\begin{tabular}{llr}
\hline 2 & Methods & $\mathbf{2 7}$
\end{tabular}

$2.1 \quad$ Mathematical background $\ldots \ldots \ldots \ldots \ldots \ldots$. . . . . . . . . . . . 27

$2.1 .1 \quad$ Single cell dynamics $\ldots \ldots \ldots$. . . . . . . . . . . . 28

$2.1 .2 \quad$ Bi-domain description of cardiac tissue . . . . . . . . . . . . . . . . 29

2.1.3 Mono-domain descriptions of cardiac tissue . . . . . . . . . . . . . 31

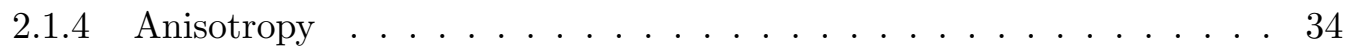

2.1 .5 The phase-field method . . . . . . . . . . . . . . . . . 34

2.1 .6 Models . . . . . . . . . . . . . . . . . . . . . . . . . . 42

2.1 .7 Spiral tips and phase singularities $\ldots \ldots \ldots$. . . . . . . 47

2.1 .8 Lyapunov stability analysis $\ldots \ldots \ldots$. . . . . . . . . . . . . . . 49

2.2 Numerical implementation $\ldots \ldots \ldots \ldots \ldots$. . . . . . . . . . . . . 57

2.2 .1 Time stepping scheme $\ldots \ldots \ldots \ldots \ldots$. . . . . . . . . . . . 57

$2.2 .2 \quad$ Diffusion Term . . . . . . . . . . . . . . . . . . . . . . 58

2.2 .3 Boundary conditions . . . . . . . . . . . . . . . . . 62

2.2 .4 Stability considerations . . . . . . . . . . . . . . . . 63

$2.2 .5 \quad$ Spiral tip detection . . . . . . . . . . . . . . . . 65

$2.2 .6 \quad$ Lyapunov exponents and vectors . . . . . . . . . . . . . . 66

2.2 .7 Hardware, software, parallelization . . . . . . . . . . . . . . . . . 69

2.3 Experimental methods . . . . . . . . . . . . . . . . . 71

$2.3 .1 \quad$ Setup and tissue preparation $\ldots \ldots \ldots \ldots 71$

2.3 .2 Optical imaging $\ldots \ldots \ldots \ldots \ldots \ldots$. . . . . . . . . . . . . . . 72

2.3 .3 Electric-field stimulation experiments $\ldots \ldots \ldots$. . . . . . . . 73

2.3 .4 Signal processing - Activation maps . . . . . . . . . . . . . 75

\begin{tabular}{lll}
\hline 3 & Results & $\mathbf{7 9}$
\end{tabular}

3.1 Quantification of dynamical complexity in heterogeneous excitable media . 79

3.1 .1 Plane waves . . . . . . . . . . . . . . . . . . . 80

3.1 .2 Rigidly rotating spiral waves $\ldots \ldots \ldots$. . . . . . . . . 87

$3.1 .3 \quad$ Multiple spiral waves . . . . . . . . . . . . . . . . . . . . . . . 91

3.1.4 $\quad$ Transition to meandering $\ldots \ldots \ldots \ldots$. . . . . . . . . . . . 95 
3.1 .5 Circular heterogeneities . . . . . . . . . . . . . . . . 97

3.1 .6 Random heterogeneities . . . . . . . . . . . . . . . . . . . . . . 101

3.1 .7 Heterogeneities in spatio-temporal chaos . . . . . . . . . . . . . . . . 105

3.1 .8 Brief summary . . . . . . . . . . . . . . . . . . . . . 113

3.2 Sensitivity of curved tissue boundaries to electric-field stimulation . . . . . 115

3.2 .1 Theoretical framework . . . . . . . . . . . . . . . . 115

3.2 .2 Setup of numerical simulations . . . . . . . . . . . . . 117

3.2.3 Generic properties of induced membrane potential changes . . . . 118

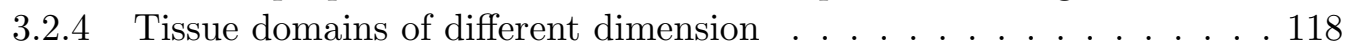

$3.2 .5 \quad$ Curvature dependence in cell culture experiments . . . . . . . . . 120

3.2 .6 Definition of boundary curvature . . . . . . . . . . . . . . . 121

3.2 .7 Flat boundaries . . . . . . . . . . . . . . . . . . . . . . . . . . 121

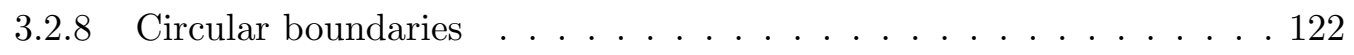

3.2 .9 Semi-circular protuberances . . . . . . . . . . . . . . . . . 129

3.2 .10 Parabolic boundaries . . . . . . . . . . . . . . . . . . . . . . . 130

3.2 .11 Inherently three-dimensional boundaries . . . . . . . . . . . . . 132

3.2 .12 Boundary effects in full numerical simulations . . . . . . . . . . . 133

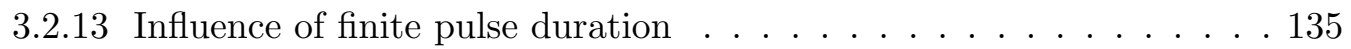

3.2 .14 Brief summary $\ldots \ldots \ldots \ldots$. . . . . . . . . . . . . . . . 141

3.3 Heterogeneity-induced wave sources in low-energy defibrillation . . . . . . . 144

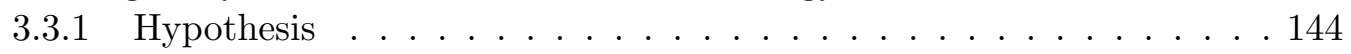

3.3 .2 Theoretical framework . . . . . . . . . . . . . . . . . . . . . . 145

3.3 .3 Blood vessel size distributions . . . . . . . . . . . . . . . . . . . . . . 149

3.3 .4 Activation times . . . . . . . . . . . . . . . . . . . 150

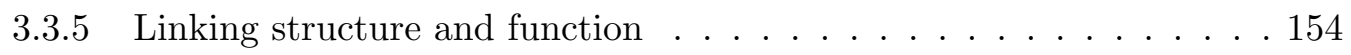

3.3 .6 Universality of activation time scaling . . . . . . . . . . . . 157

3.3 .7 Brief summary . . . . . . . . . . . . . . . . . . . . 159

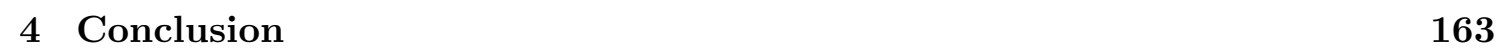

4.1 Summary . . . . . . . . . . . . . . . . . . . . . . . . . 163

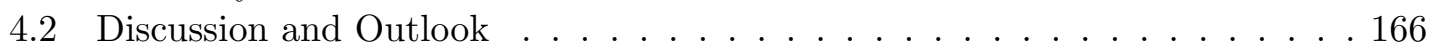

4.3 Concluding remarks $\ldots \ldots \ldots \ldots$. . . . . . . . . . . . . . . . . . . . .

\begin{tabular}{|ll}
\hline A Modeling details & 177
\end{tabular}

\begin{tabular}{ll}
\hline B Supplementary data & 179
\end{tabular}

\begin{tabular}{|lll}
\hline C & MediaSim - an open framework for simulating extended systems & 183
\end{tabular}

$\begin{array}{ll}\text { Index } & 186\end{array}$

\begin{tabular}{ll}
\hline List of Figures & 187
\end{tabular}

$\begin{array}{lr}\text { List of Tables } & 189\end{array}$

\begin{tabular}{lr}
\hline Bibliography & 191 \\
\hline
\end{tabular} 


\section{Abbreviations}

\begin{tabular}{|c|c|}
\hline $\mathrm{APD}$ & action potential duration \\
\hline ATP & antitachycardia pacing \\
\hline $\mathrm{CV}$ & conduction velocity \\
\hline ECG & electrocardiogram \\
\hline FTCS & forward-time centered-space \\
\hline ICD & implantable cardioverter-defibrillator \\
\hline LEAP & low-energy anti-fibrillation pacing \\
\hline$\mu \mathrm{CT}$ & mirco-computed tomography \\
\hline ODE & ordinary differential equation \\
\hline $\mathrm{PDE}$ & partial differential equation \\
\hline $\mathrm{PDF}$ & probability density function \\
\hline $\mathrm{RDE}$ & reaction-diffusion equation \\
\hline
\end{tabular}




\section{Chapter 1}

\section{Introduction}

Since the Enlightenment, the natural sciences have been undergoing a process of immense diversification and progress. The gain of knowledge has led to the formation of more specialized fields, namely physics, chemistry and biology, which have themselves diversified into a vast number of subfields in the course of the twentieth century. However, the continuing improvement of theoretical and experimental techniques has, at the same time, fostered interactions between subjects which had been regarded before as very distant relatives in this genealogy of fields. Examples are the emergence of "(bio)physical chemistry", "biophysics" or "biomedical engineering". The name of the group within which the work for this thesis was carried out is "Biomedical Physics", indicating its focus of research at the interface of physics, biology and medicine.

During the last decades, the focus of interest of many physicists has started to include phenomena observed in living matter - from molecules to cells, organisms and populations. The reason for this development is at least twofold: on the one hand, biological investigations have revealed more and more microscopic detail of biological processes. Since all these processes ultimately obey the laws of physics (which might not be obvious on a macroscopic scale), there is a natural demand for physicists to determine the underlying physical basis. Prominent examples of active research interests in this area are protein folding dynamics [1, physics of the cell membrane and membrane proteins [2], ligand-receptor interactions [3] and other protein-protein interactions such as kinesin-microtubule binding [4] or actin-myosin cross-bridging [5]. On the other hand, the continuously improving methodology and the abstract concepts of physics and even mathematics have been found to be applicable to biological systems on higher levels, especially for the description of emergent properties of so-called complex systems. The term refers to a self-organizing, composite system displaying a collective behavior which is not obviously visible in any of its many constituent parts. Non-linearity plays an important role for such emergent behavior, in that it invalidates the superposition principle. Under these considerations, physicists from the field of non-linear dynamics and complex systems have been studying a wide range of phenomena in networks of neurons [6], genetic networks [7, multicellular organization [8], epidemic spread in populations [9] and evolutionary dynamics [10], to only name a few examples from biology.

The perspectives described above from which physicists investigate biological systems represent two distinct approaches: in the first case, the goal is to understand the detailed inner workings of specific biological components from first principles, i.e. from the fun- 
damental laws of physics. For example, this is done in large-scale molecular dynamics simulations, where thousands of atoms forming a protein and its environment follow the action of theoretical, semi-empirical or empirical inter-molecule force fields to study protein dynamics and stability on the nanosecond timescale [2]. The second approach relies more on generic properties of certain biological systems which make them fit into a specific framework. For instance, interacting biological oscillators (which may be cells, animals or more abstract processes [11]) often exhibit synchronization phenomena, whose characteristics can be studied without knowing how the individual oscillators function in detail (see reference [12] for an example of synchronized chirps in crickets). Naturally, this results in a more descriptive manner of approaching the system in question, but sometimes also leads to useful analogies between different realizations of the same fundamental phenomena. There is, of course, also the possibility to gradually add a certain level of detail to the generic description of specific phenomena. But in contrast to the first, bottom-up approach, this merely happens in a top-down manner.

The work of this thesis falls into the second category of the generic approach to biological systems. In a first approximation, most of the muscle constituting the heart can be viewed as a so-called excitable medium. In general, excitable systems are characterized by a few fundamental properties:

- There is a stable resting state, meaning that small perturbations quickly decay without exciting the system.

- In response to a (still small but) strong enough perturbation, upon crossing an excitation threshold, the system displays a characteristic course of dynamics, called an activation.

- Without any further input, the system eventually returns to the excitable resting state, but only after going through a phase in which excitability is reduced or completely lost. This time span is called the refractory period.

If many of such excitable units are spatially coupled, they form an excitable medium, which supports the propagation of non-linear excitation waves. Due to the above local properties of the medium, these waves differ in some essential aspects from the optical or acoustic waves observed in passive media: As a result of the excitation threshold, it is usually not sufficient to induce excitation in a single excitable element in order to produce a propagating wave, but a finite excited area is required, whose size depends on the coupling strength. After some transient propagation, the wave profile is usually independent of the method used to initiate the wave. This behavior is inherited from the characteristic form of the activation in a single element, which is somewhat altered by the coupling but generally independent of the exciting stimulus. A more critical factor determining the response to a stimulus is the refractoriness due to preceding activations of the same site. One of the most striking non-linear wave phenomena in excitable media is that of annihilation: When two wave fronts collide, the refractory tail behind each wave prohibits propagation of activity in the direction of either wave, leading to the annihilation of the two. This basic mechanism has important consequences for two competing wave sources exciting the medium at different frequencies: Assuming both sources produce propagating wave trains at the corresponding frequencies, the domain of influence of the faster wave 
a)

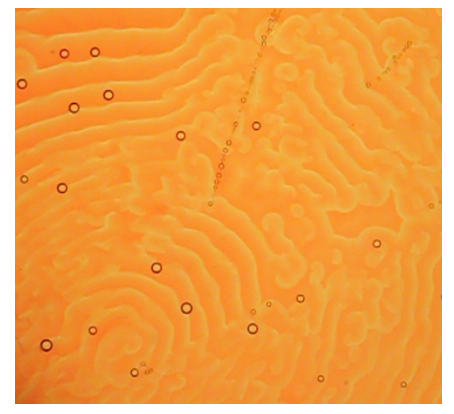

b)

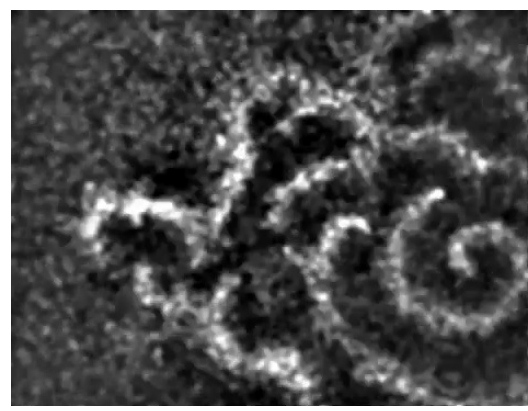

c)

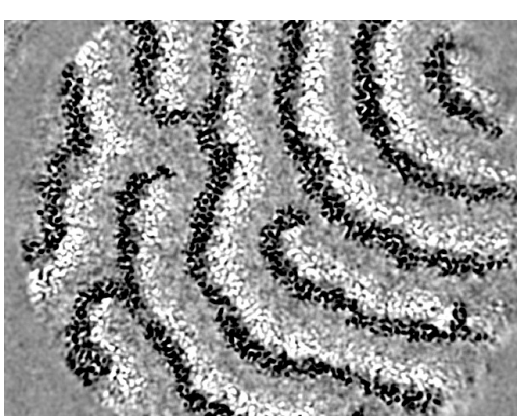

Figure 1.1: Spatio-temporal patterns in different excitable media. a) Chemical waves in the two-dimensional Belousov-Zhabotinsky reaction. Waves originating from the lower left corner have been broken by disturbing the solution in the petri dish with a pipette. Picture by C. Richter. b) Spiral wave patterns in a colony of starving dictyostelium discoideum (difference beetween two consecutive images, picture by D. LOH and A. BAE). c) Contraction waves in cultured cardiomyocytes from neonatal rats, made visible by difference imaging. Picture by C. RICHTER.

source will continually grow due to wave annihilation at the domain boundary, until the whole medium is controlled by the faster source.

Depending on the level of detail one is interested in, one excitable unit in the heart corresponds to a piece of cardiac muscle cell membrane, one cell or a small tissue region. As will explained below, the excitable nature of cardiac tissue is the basis for its normal function, but the generic mechanisms present in all excitable media - like the aforementioned high-frequency domination - also play an important role when the rhythm of the heart is disturbed. A number of substrates other than cardiac tissue can, under the right conditions, exhibit the characteristics of an excitable medium. These are mostly chemical and biological systems, including the Belousov-Zhabotinsky reaction [13, 14] (investigated by 1980 Lenin Prize laureates B. P. Belousov and A. M. Zhabotinsky), catalytic CO oxidation on platinum surfaces [15] (the system involved in the studies that led to the award of the Nobel Prize to G. ERTL), collective cell dynamics in the aggregation of the slime mold dictyostelium discoideum [16] and mammalian cortical tissue [17]. Wave patterns observed in those systems range from regular, periodic patterns to self-sustained spiraling wave activity to spatio-temporal chaos. Examples of the non-linear waves observed in some of the above systems are shown in figure 1.1, including activity in cell cultures of cardiomyocytes, which is the main cell type in the cardiac muscle. Before defining the central questions and hypotheses of this thesis in section 1.7, it is useful to review briefly 


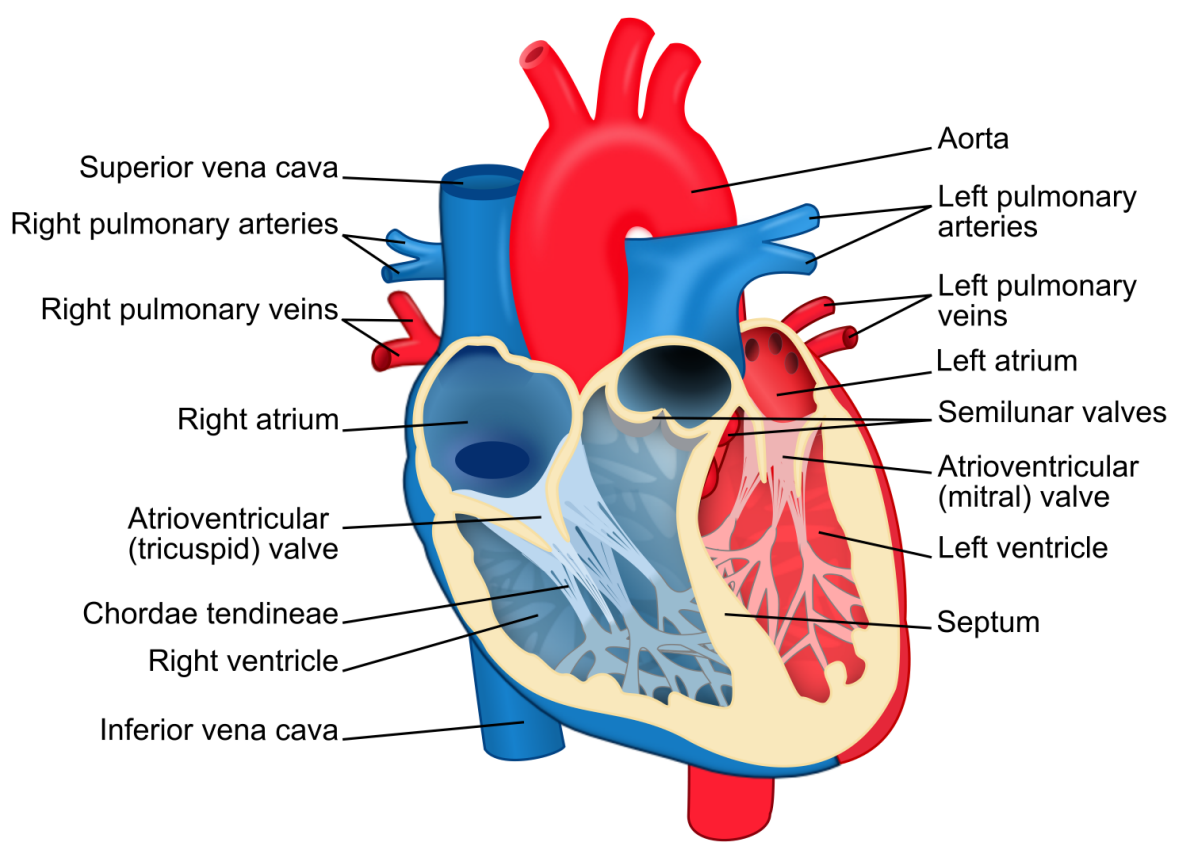

Figure 1.2: Anatomy of the heart. Cavities and vessels transporting oxygenated blood are colored in red, those filled with oxygen-depleted blood are marked blue. Picture modified from [19].

- from a physicist's point of view - the anatomy and physiology of the heart. On the one hand, this intermezzo is meant to give the reader the opportunity to familiarize with the physiological properties of the heart which give rise to a system that can indeed be caricatured as an excitable medium. On the other hand, the aspects of the heart will be introduced which exceed the generic description as an excitable medium and are necessary to answer specific questions, foremost its structural complexity and the specific medical means of controlling its activity.

\subsection{Anatomy of the heart}

The heart is the central pumping organ for blood in all higher organisms and drives the circulatory system that is designed to supply all body tissues with adequate amounts of oxygen and nutrients and to remove carbon dioxide and other waste products. In humans, it is a four-chambered hollow muscle (see figure 1.2), which, by coordinated contraction, transports de-oxygenated blood to the lungs and oxygenated blood to other organs including the extremities of the body 1 The four chambers consist of two ventricles, each receiving fluid inflow from its adjacent atrium during one heart beat. A system of valves ensures that, during the sequence of contractions (systole) and relaxations (diastole) of the different chambers commonly referred to as the cardiac cycle, blood is taken in from the veins and pumped out through the arteries. The right heart (the right atrium and ventricle as a whole) is responsible for the pulmonary circulation, receiving oxygen-depleted blood from the body and releasing it through the right ventricle into the pulmonary arteries.

\footnotetext{
${ }^{1}$ As a reference for the basic cardiac anatomy described in the following, see, e.g., reference [18.
} 
After the lung has replenished the blood with oxygen, it enters the left heart through the left atrium and is pumped out through the heart's biggest artery, the aorta, which is connected to the left ventricle. One of the amazing properties of the heart is that it also supplies blood to its own tissue: Just outside the aortic valve, the left and right coronary arteries branch off from the aorta. They run in between the pericardium, a protective sac for the heart, and the epicardium, the outermost layer of actual muscle tissue (myocardium). From there they branch off into the myocardium, splitting into smaller and smaller vessels which provide the necessary blood supply. A similar tree-like structure is formed by the coronary veins which remove oxygen-depleted blood and waste products from the muscle.

The two atrioventricular valves separating the atria and the ventricles are connected via the cordae tendineae to the papillary muscles and trabeculae of the ventricles. The former are large-scale finger-shaped protrusions from the bulk of ventricular muscle tissue, while the latter are beam-like muscle strands, connected to the bulk tissue in different places. They contract together with the ventricles and thereby pull on the cordae tendineae, thus preventing valve prolapse into the atrium due to the high pressure during the ventricular systole.

\subsection{Physiology of the heart}

The cardiac cycle is controlled by electrical signals traveling through the heart $2^{2}$ The phenomenology of the heart's bioelectricity has been known since the end of the nineteenth century, when time-varying potential differences on the body surface were discovered which are correlated with the heart beat. They were made practically available as electrocardiograms (ECGs) for diagnosis by W. EINTHOVEN through his invention of the electrocardiograph, for which he received the Nobel Prize in 1924. On the microscopic scale, these electrical signals are known today to correspond to action potentials performed by individual muscle cells (cardiomyocytes), similar to those of neurons (with some important qualitative differences, see section 1.2.1). The pathway for excitation from initiation to the contraction of the ventricles is shown in figure 1.3 . In the sinoatrial node located in the right atrium, specialized pacemaker cells spontaneously generate action potentials, which, under normal physiological conditions, are considered the start of the cardiac electrical conduction system. Action potentials are rapid transient alterations of a cell's transmembrane voltage. The rate at which the pacemaker cells depolarize defines the heart rate and is influenced by the autonomous nervous system. The sinoatrial node directly excites the muscle tissue of the right atrium from which the activity can propagate to the left atrium via different connections, the fastest of which is usually Bachmann's bundle. Any activity spreading to the ventricles has to pass through the atrioventricular node located at the top of tissue separating the two ventricular chambers of the heart, the septum. The atrioventricular node delays the conduction of the signal by about $100 \mathrm{~ms}$, ensuring the ventricles are filled before contraction occurs. Additionally, it is the first in a cascade of (slower) backup pacemakers which can initiate contraction of the ventricles in case the

\footnotetext{
${ }^{2}$ As a reference for the brief overview of cardiac electrophysiology given in the following, see, e.g., reference [20].
} 


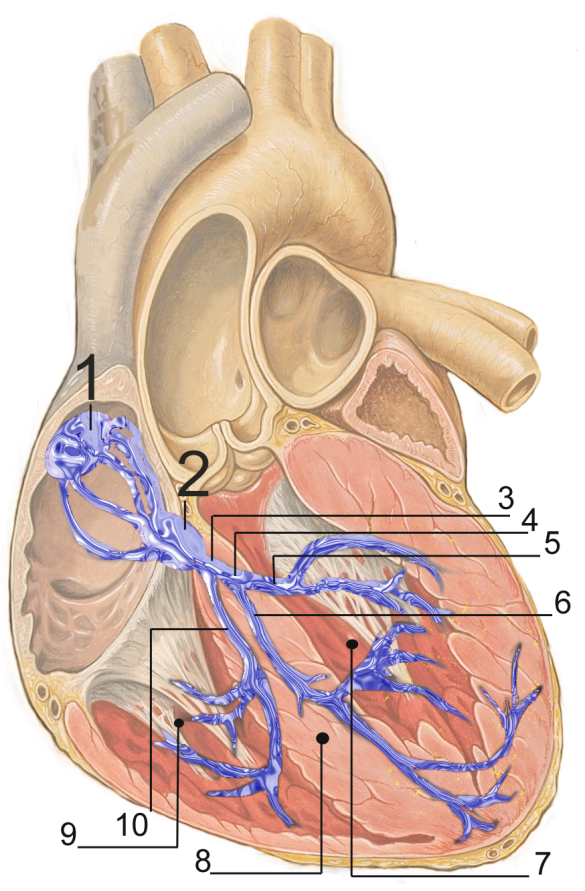

Figure 1.3: Electrical conduction system of the heart. (1) Sinoatrial node; (2) Atrioventricular node; (3) Bundle of His; (4) Left bundle branch; (5) Left posterior fascicle; (6) Left-anterior fascicle; (7) Left ventricle; (8) Ventricular septum; (9) Right ventricle; (10) Right bundle branch. Picture from [21].

input from the sinoatrial node is absent or blocked ${ }^{3}$ From the atrioventricular node, the His bundle and the Purkinje fibers conduct action potentials down the septum and to the left and right ventricles. The fibers of the electrical conduction system consist of specialized cardiomyocytes and are connected to the actual contracting tissue only in a few places. Within the bulk of tissue, the activity propagates via gap junctions connecting individual cardiomyocytes to their neighbors. The arrangement of excitatory connections between different parts of the heart therefore coordinates the activation sequence, which is indispensable for the heart to effectively fulfill its function.

\subsubsection{Cardiomyocytes}

Like all cells, cardiomyocytes in their resting state have a finite membrane voltage or potential, which is defined as the electrical potential within the cell with respect to the extracellular space. It arises from the interaction of electrical and ion concentration gradients across the cell membrane. Inside the cell, the concentration of $\mathrm{Na}^{+}, \mathrm{Ca}^{2+}$ and $\mathrm{Cl}^{-}$ ions is much lower than in the extracellular fluid, while the converse is true for $\mathrm{K}^{+}$ions. These different ion concentrations are maintained by active membrane proteins including the $\mathrm{Na}^{+} / \mathrm{K}^{+}$ATPase and $\mathrm{Ca}^{2+}$ ATPase, which derive their energy from the hydrolysis of adenosine triphosphate. The fact that, under normal physiological conditions, the ion concentrations are stable is called ion homeostasis.

\footnotetext{
${ }^{3}$ In fact, the ventricles themselves can initiate an activation due to the tendency of all cardiomyocytes to depolarize spontaneously. However, the intrinsic rate of this last component in the backup cascade is very slow (20 to 40 beats per minute). Therefore, idioventricular rhythm is only observed if all other pacemakers fail.
} 
For a single ion species, the difference in concentration across the membrane causes a directed net flow of ions that seeks to compensate the concentration gradient, provided the membrane has a non-zero permeability. However, since the diffusing particles are charged, this leads to the buildup of an electrical gradient, counteracting the concentration gradient. The electrical potential difference between the intracellular and the extracellular space, when these two opposing gradients balance, is called the reversal potential of the ion species. It can be calculated from the Nernst equation (see reference [20], pp. 78-83)

$$
V_{\mathrm{x}}=\frac{k_{B} T}{e z} \ln \frac{c_{\mathrm{x}}^{\mathrm{ex}}}{c_{\mathrm{x}}^{\mathrm{in}}}
$$

where $\mathrm{x}$ refers to the ion species, $c_{\mathrm{x}}^{\mathrm{ex}}$ and $c_{\mathrm{x}}^{\text {in }}$ are its extracellular and intracellular concentrations, respectively, $k_{B}$ is Boltzmann's constant, $e$ is the elementary charge and $z$ is the degree of ionization of the ion species. Since the ion concentrations are more or less constant due to ion homeostasis, each ion species has a fixed reversal potential. Using equation (1.1), these evaluate to approximately $-90 \mathrm{mV}$ for $\mathrm{K}^{+},+60 \mathrm{mV}$ for $\mathrm{Na}^{+},-80 \mathrm{mV}$ for $\mathrm{Cl}^{-}$and $+120 \mathrm{mV}$ for $\mathrm{Ca}^{2+}$.

When multiple permeating ion species are present, the resulting resting potential can be calculated using the Goldman-Hodgkin-Katz equation [22, 23], in which the concentrations $c_{\mathrm{x}}$ of equation (1.1) are replaced by weighted sums for the different ion species and the weights are given by the corresponding membrane permeabilities 4 Specialized pores in the cell membrane, so-called ion channels, selectively determine the permeability of the membrane for different ion species. At rest, the membrane is mainly permeable to $\mathrm{K}^{+}$, which is why the resting membrane potential of a cardiac cell is between $-90 \mathrm{mV}$ and $-80 \mathrm{mV}$.

The permeability of the membrane for individual ion species can change rapidly, because ion channels are active elements that can open and close in response to external stimuli. This mechanism of ion channel gating is what gives the membrane potential its dynamics and the cell the ability to perform action potentials. A schematic sketch of the cardiac action potential is shown in figure 1.4. A small depolarization of the cell above a critical threshold between $-70 \mathrm{mV}$ and $-60 \mathrm{mV}$ increases the opening probability of voltage-gated sodium channels and initiates a positive feedback process: the increased permeability drives the membrane potential more towards the reversal potential of sodium, which leads to the opening of even more sodium channels. Since the $\mathrm{Na}^{+}$ions move down both the electrical and concentration gradient, this process results in a rapid upstroke with an amplitude of approximately $100 \mathrm{mV}$ on a millisecond time scale.5 after which most sodium channels are inactivated. During the upstroke, around $-25 \mathrm{mV}$, voltage-gated calcium channels in the membrane slowly start to open. After a small but rapid repolarization caused by a transient outward $\mathrm{K}^{+}$current, the influx of $\mathrm{Ca}^{2+}$ into the cell, combined with decreased $\mathrm{K}^{+}$conductance and calcium-induced calcium release from internal storages such as the sarcoplasmatic reticulum, leads to the formation of a plateau phase of the membrane potential which lasts about $200 \mathrm{~ms}$ and is one of the main differences to

\footnotetext{
${ }^{4}$ The equation can also be generalized to include ion species of differing ionization degrees. For a summary of extensions, see reference [24].

${ }^{5}$ The time scale differs greatly across different cell types. For example, pacemaker cells in the sinoatrial node depolarize more slowly than the average myocyte in the bulk of ventricular myocardium, while the converse is true for Purkinje cells.
} 


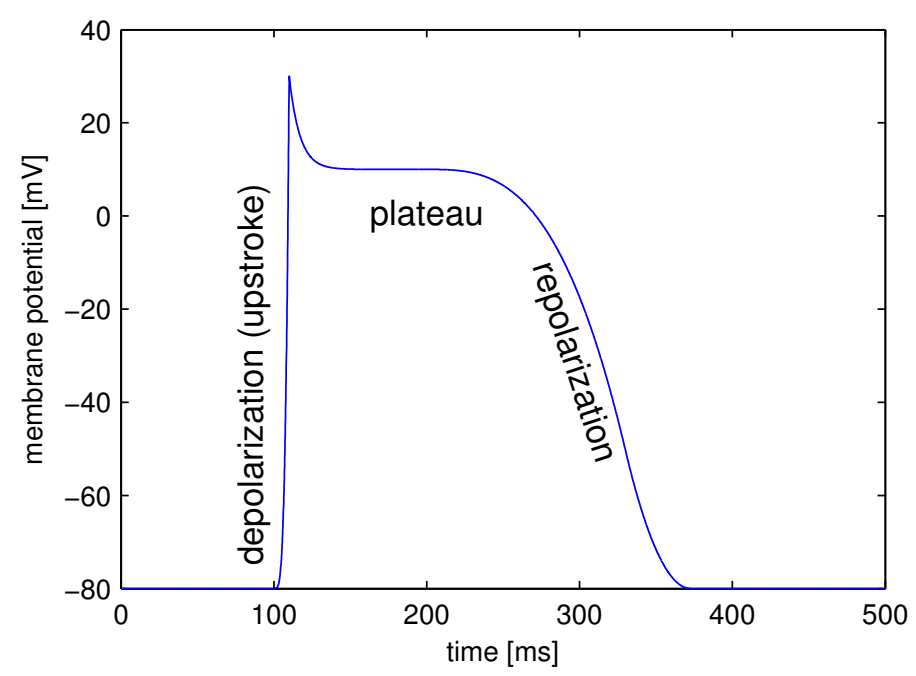

Figure 1.4: Cardiac action potential.

other excitable cells such as neurons in the brain. Towards the end of the plateau, the potassium conductance increases again and the calcium channels close, which causes the cell to repolarize to its resting membrane potential. Only now, triggered by the value of the membrane potential, the sodium channels return from their inactivated to the (initial) closed state, which can be activated again to elicit another action potential. The initial persistence of the inactivated state is what causes the cell to be less excitable after an action potential has been initiated and is therefore mainly responsible for the refractoriness of the cell as an excitable system. In addition to the aforementioned ATPases, the $\mathrm{Na}^{+} / \mathrm{Ca}^{2+}$ exchanger in the membrane helps expelling excess calcium from the cell. Most of the calcium is, however, taken up by the sarcoplasmatic reticulum via the $\mathrm{Ca}^{2+}$ ATPase. For a detailed description of the contribution of individual ion channels and their gating mechanisms beyond the simplified picture given here, the reader be referred to an extensive review by A. O. Grant [25].

The macroscopic force of the cardiac muscle is caused on the microscopic scale by the contractile force of individual cardiomyocytes, which is produced by the interaction of myosin and actin filaments within the cells. The action of this contraction machinery is triggered by the high intracellular calcium concentrations arising during the plateau phase of the action potential (excitation-contraction coupling [26, 27]) and is delayed with respect to the electrical activity of the cell.

\subsubsection{Cell-to-cell coupling}

Activity initiated by the excitation conduction system introduced at the beginning of section 1.2 can propagate from cell to cell in order to cause contraction of a macroscopic region of the muscle. This cell-to-cell coupling stems mainly from gap junctions - proteins providing a direct electrical connection between the intracellular spaces of two neighboring cells [28] $]^{6}$ In this way, one cell performing an action potential can provide the necessary

\footnotetext{
${ }^{6}$ There is evidence that field effects provide an additional contribution to excitation conduction in cardiac tissue [29].
} 
superthreshold stimulation for its neighboring cells.7 From a viewpoint of a reduced modeling approach, this is a next-neighbor coupling scheme which leads, macroscopically, to a diffusive interaction and thus turns the cardiac muscle into an excitable medium. As cells surrounding an excited cell not only receive input from that cell but also act as a charge sink, the action potential shape during wave propagation is altered compared to that measured in isolated cardiomyocytes [31].

\subsection{Structural Heterogeneity}

The coupling between cells in cardiac tissue is inherently anisotropic, due to both the elongated, cylindrical shape of individual myocytes and the inhomogeneous distribution of gap junctions, which are found preferentially at the ends of the cell pointing in the longitudinal direction [28]. The anisotropic behavior of the tissue as a whole stems from the tendency of myocytes to locally align with their neighbors. However, this fiber orientation is not constant throughout the muscle, but rotates continuously from the endocardium to the epicardium by about $180^{\circ}$ in total. Additionally, the cardiac muscle is organized in layers, so-called sheets, which are separated by regions of reduced coupling [32]. In the healthy heart, the anisotropy of the bulk electrical conductance manifests first of all in the anisotropy of conduction velocity [33]. Apart from the intracellular coupling, additionally, the ionic, i.e. local, properties of cardiac cells are known to differ across the ventricular wall and in different regions of the cardiac muscle, including dispersion in action potential duration and repolarization [34, 35].

The anatomy of the heart implies that a certain level of complexity and heterogeneity is always present: As described in section 1.1, the coronary vasculature supplies the tissue with blood and removes waste products. The vessels form a tree-like network comprising the full range from very large vessels down to the smallest capillaries. An illustrative image obtained from mirco-computed tomography $(\mu \mathrm{CT})$ is shown in figure 1.5. Where these vessels penetrate the tissue, they disrupt the intracellular coupling between myocytes and therefore form non-conducting inclusions in the tissue, which can also be viewed as internal tissue boundaries. Other complex boundary shapes are introduced on the ventricular endocardium by surface undulations, papillary muscles and trabeculae (see section 1.1 and figure 1.6.

Besides this rather necessary and intended complexity of the cardiac muscle, further heterogeneity in the system is caused by random impurities such as fatty tissue, scars and fibrotic tissue, which may grow in number and intensity in the process of cardiac remodeling after myocardial infarction or due to other diseases. Fibroblasts occur naturally in healthy tissue as they are responsible for maintaining the extracellular matrix in which the cardiomyocytes are embedded. However, it has become clear that they not only influence the coupling between cardiomyocytes during development and tissue repair, but are also directly coupled to cardiac muscle cells and can therefore change the electrophysiological behavior of the tissue by acting as current sinks [36, 37].

\footnotetext{
${ }^{7}$ As mentioned at the beginning, as for generic excitable media, one activated cell alone is usually not enough to excite its neighbors (see introduction of reference 30. for a summary).
} 


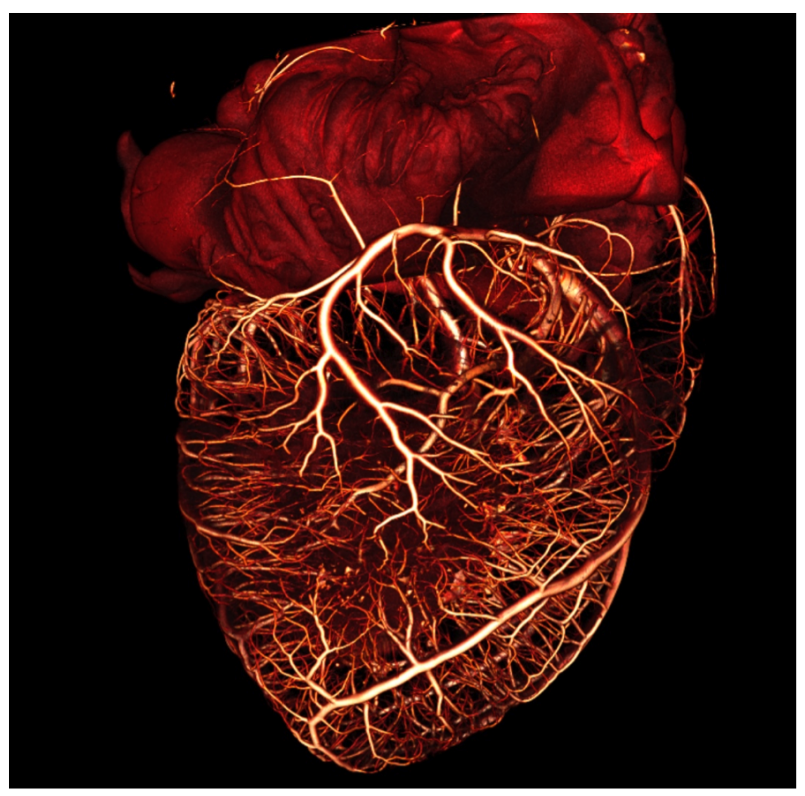

Figure 1.5: The tree of cardiac vessels. Visualization of the branching network of coronary vessels of a whole beagle dog heart from a $\mu \mathrm{CT}$ scan after injection of contrast agent. The diameter of the heart is approximately $6.5 \mathrm{~cm}$. Visualization and $\mu \mathrm{CT}$ data by M. L. RICCIO/Cornell University $\mu \mathrm{CT}$ Facility for Imaging and Preclinical Research and F. H. FENTON/Cornell University.

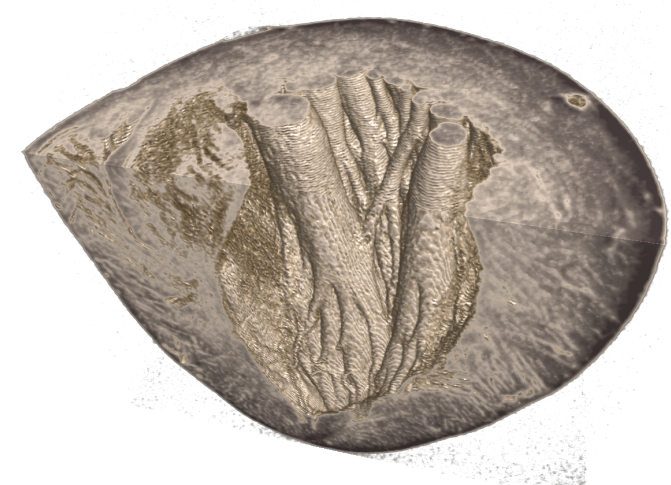

Figure 1.6: Endocardial structure. Complex geometry of the inner ventricular wall of a mouse ventricle with a diameter of approximately $5 \mathrm{~mm}$. Visualization and $\mu \mathrm{CT}$ data by D. Hornung. 


\subsection{Arrhythmias}

Failure of the cardiac muscle to produce a normal heart beat or normal sinus rhythm can originate from different parts of the electrical conduction system of the heart. Besides the inability of the fast fibers to excite specific parts of the muscle (AV-block, bundle blocks, etc.), one important source of arrhythmias is the bulk of cardiac muscle tissue itself. Under normal conditions, the activation of a chamber of the heart can be caricatured as a coherent wave front, traveling across the tissue in a quasi-planar manner and thereby exciting the whole chamber. Afterwards, the muscle recovers and awaits the next triggered wave. However, waves that do not fully excite the available tissue volume at the first attempt can lead to the formation of reentrant wave patterns [38, 39, 40] (similar to those shown in figure 1.1. . Once these self-excited spiral waves, or scroll waves in three dimensions, have been initiated, they excite the tissue with a frequency much larger than the natural pacemaker and thereby decouple a region from the control of sinoatrial node (via the mechanisms of high-frequency domination explained at the beginning of the introduction, see also reference [41]). If the ventricles are affected, spiral waves therefore lead to an unnaturally fast heart rate, called tachycardia in medical terms. Furthermore, the pumping function of the heart is impaired, since the contraction of the ventricles ceases to be coordinated with atrial activation and not the whole ventricle contracts at once. An even more dangerous situation arises when spiral waves break up into multiple waves, which may lead to a state of spatio-temporal chaos that is known as ventricular fibrillation [42, 43]. Under these conditions, small tissue regions contract in arbitrary sequence, causing the heart to completely lose its pumping force. The result is a standstill of circulation, called cardiac arrest, and consequently damage to the heart tissue itself, to other organs and, irreversibly within minutes, to the brain. Ventricular fibrillation thus is a lethal state of cardiac activity and its spontaneous occurrence is one of the main reasons for sudden cardiac death, which accounts for about 500,000 annual deaths in the United States [44]. Fibrillation can also occur in the atria, where it is not immediately life-threatening due to the filter effect of the atrioventricular node, which imposes an upper frequency limit on the excitation signal transmitted to the ventricles. However, even atrial fibrillation can cause serious long-term damage, e.g., by increasing the risk of stroke to five times its normal value [45].

The initiation mechanisms for arrhythmias are an active area of research. Most of the proposed mechanisms have in common that due to specific conditions, waves are partially blocked and some tissue region remains excitable for the potential formation of a reentry. One way this is possible is through premature beats (extrasystoles), where the partial block is caused by dispersion in refractoriness due to the preceding normal activation, acting as a dynamical heterogeneity. Alternatively, the substrate may have spatially inhomogeneous excitability, and this structural heterogeneity (see section 1.3 can cause a partial block under stress conditions. For both possibilities, a number of underlying reasons have been identified, including, but not limited to, genetic mutations altering the dynamics of ion channels or receptors [46, 47, 48, remodeling after myocardial infarction [49, 50], electrical shocks during the vulnerable phase of a normal heart beat [51, 52, 53, 54] or fibrosis [55, 56, 57]. A case which is difficult to classify is idiopathic ventricular fibrillation, which is thought to be responsible for a considerable percentage of sudden cardiac deaths and 
occurs in seemingly healthy patients [58].

\subsection{Antiarrhythmic therapies}

There are at least three ways of treating cardiac arrhythmias: pharmaceutical intervention, ablation and electric shocks. While the first two can be used to reduce the susceptibility of the cardiac muscle to undesirable activation patterns, the latter cannot prevent arrhythmias from occurring. However, electrical defibrillation is the only therapy available for acute lethal arrhythmias such as ventricular fibrillation. Antiarrhythmic drug therapy can help controlling non-life-threatening arrhythmias or reduce the risk of arrhythmia onset, in particular for patients after myocardial infarction or suffering from atrial fibrillation [59, 60. In some cases, a specific region in the heart can be identified as the cause of recurring arrhythmias. For example, certain types of atrial fibrillation have been shown to be caused by focal activity originating at the junction of the pulmonary veins and the left atrium. In radio-frequency ablation, a high-intensity current is used to deliberately create lesions around the foci and thereby electrically decouple the responsible region from the rest of the tissue [61, 62]. The same strategy is also applied to the ventricles, where areas that are "critical to the arrhythmia" [63] are destroyed. Apart from strong side effects of antiarrhythmic drugs that can damage other organs and possible (undeliberate) lesions of the heart from the ablation procedure, both antiarrhythmic drug medication and ablation bear the risk of proarrhythmic effects [64, 65, 66]. This is probably due to the indication-based prescription of specific drugs and the phenomenological choice of ablation sites, which are only rarely supported by a theoretical understanding of the mechanisms. In cases where proarrhythmic effects cannot be ruled out or if there is an irreducible risk of recurring ventricular arrhythmias, it is therefore appropriate to provide a "safety net" with the implantation of a device that can automatically deliver appropriate electrical shocks to terminate an arrhythmia [67].

To terminate the most lethal form of arrhythmia, ventricular fibrillation, implantable cardioverter-defibrillators (ICDs) as well as external defibrillators deliver a short, highenergy electrical shock ${ }^{8}$ This shock ( $\sim 300 \mathrm{~J}$ within $10 \mathrm{~ms}$ if applied externally) is thought to excite the whole tissue, thus depriving the chaotic reentrant waves of the excitable substrate needed for propagation. In this way, all waves are terminated indiscriminately and the heart is shocked into quiescence. Afterwards, the low-frequency impulses of the sinoatrial node (see section 1.2) can excite the heart and restore normal sinus rhythm. There is contradictory evidence whether the shock energies used in today's defibrillators can cause considerable injury of the cardiac muscle [69, 70] and possibly increase the risk of future arrhythmias by introducing additional structural heterogeneity. A second important problem is that ICD therapy is also associated with severe psychological implications such as anxiety and reduced quality of life [71, mainly due to defibrillating shocks delivered during consciousness. These can be triggered due to early or mis-detection and are a traumatic pain experience for patients with implanted devices. Therefore, there is a search for low-energy alternatives to conventional defibrillation.

\footnotetext{
${ }^{8}$ For slower arrhythmias such as ventricular tachycardia, there are low-energy alternatives such as antitachycardia pacing (ATP) 68.
} 


\subsection{Complexity in structure and dynamics}

As outlined above, the heart is a complex system in terms of structure, owing to its anatomy and heterogeneous bulk properties, as well as in terms of the (malignant) dynamics it exhibits. Obviously, the structural complexity forms the substrate for the dynamics, which is why the two kinds of complexity can interact across the whole range from the triggering of reentrant wave patterns, to their stabilization or further degeneration into spatio-temporal chaos, to their termination via pharmaceutical or electrical intervention. The dynamical complexity is thus only partly a result of the behavior as a generic excitable medium: planar waves representing normal activation, spiral waves associated with tachycardia and spatio-temporal chaos underlying cardiac fibrillation can be observed in simple, generic (and homogeneous) models of excitable media [72, 73]. The spontaneous occurrence of wave breaks necessary to produce arrhythmias in the first place, though, cannot be explained by such simplistic models. In contrast, the interaction of the dynamics with structural heterogeneities and the stochastic behavior of single cells have been shown to facilitate the occurrence of wave breaks and the development of reentrant waves [74, 75, 76, 77]. These spiral or scroll waves waves can then interact with heterogneities, e.g. by pinning to inexcitable regions in the tissue [39, 78, 79, 80, by drifting due to excitability gradients [78, 81, 82, 83] or by destabilization due to the inherent and spatially varying anisotropy of cardiac tissue [84, 85, 86].

Over the last twenty years, it has become clear that the importance of the non-uniformity of the cardiac substrate extends to the mechanisms of electrical defibrillation. The simple picture given in section 1.5 suggests that, in some way, most of the tissue is excited by a sufficiently strong electrical shock, which ultimately terminates all waves propagating through the muscle. In early efforts to understand the mechanism underlying electric-field induced activation of the tissue, this was thought to be possible because of the intracelluar coupling scheme of cardiac tissue alone, where the voltage drop at high-resistance gap junctions leads to sawtooth-shaped depolarization pattern along a chain of interconnected cells. The spikes located at gap junctions were thought to be sufficient to initiate an action potential at one end of each cell [87, 88, 89, 90]. However, several studies revealed that the expected depolarization patterns are not observed in real, well-connected tissue [91] and instead, additional heterogeneities in electrical conductance (including those on larger length scales) play a major role in the formation of these so-called secondary sources or virtual electrodes [92, 93, 94, 95, 96, 97, 98. Electrical shocks above a critical defibrillation threshold of about $6 \mathrm{~V} / \mathrm{cm}$ are believed to, on the one hand, activate a large enough tissue fraction by heterogeneity-induced virtual electrodes to terminate all existing waves and, on the other hand, minimize the risk of inducing new arrhythmias by interaction with preexisting activity [99, 100]. However, as most of the studies are focused on elucidating the mechanisms of conventional, high-energy, defibrillation, they are lacking perspectives for the development of gentler defibrillation techniques.

\subsection{This thesis}

The work presented in this thesis aims at elucidating the mechanisms of arrhythmia development and control in the heart which originate from its inherent heterogeneity and 
complex structure. The hope is that the results may open the path to the development of novel antiarrhythmic therapies which are efficient insofar as they do not disturb the normal function of the heart or damage the muscle more than necessary. This requires, on the one hand, a detailed understanding of the processes that lead to the development of arrhythmias and a way to assess the complexity of the resulting activation patterns, in order to provide tailor-made therapies for different scenarios. On the other hand, the design of such control strategies has to be supported by theoretical concepts which can predict the effect of an anti-arrhythmic intervention and thus enable the choice of appropriate parameters for the control strategy.

Three different topics are presented, which approach this problem from both of the abovementioned directions: The first one adopts the generic view of cardiac tissue and aims at establishing a quantitative stability analysis method from non-linear dynamics as a new tool in the field of modeling excitable media. The other two focus on elucidating in theory (second topic) and experiments (third topic) the mechanisms underlying future low-energy techniques for electrical antiarrhythmic therapy and specifically the role of internal and external tissue boundaries. The theoretical background and its numerical implementation necessary for the work of sections 3.1 and 3.2 as well the experimental technique of optical mapping for section 3.3 are introduced in section 2. The results presented in section 3 are ordered from generic to specific, from theoretical to experimental:

Section 3.1 is centered around the question, how the stability and dynamics of activation patterns in models of cardiac tissue can be characterized quantitatively. A very practical and common approach to obtain qualitative descriptions is to numerically simulate the dynamics and simply observe (possibly for different levels of heterogeneity or different parameters) characteristic quantities such as the action potential duration, conduction velocity, the trajectories of spiral tips and whether or not spiral waves break up [101, 102, 85, 103, 104, 74, 76]. To get a more quantitative insight and determine the mechanisms that underlie spiral wave behavior and interaction, phenomenological approaches like the definition of predator-prey quantities [105] have been proposed. In some more detail, the stability of spiral waves has been assessed using linear stability analysis [106, 107, 108] and response functions, which can be used to predict spiral wave drift [109, 81. However, both of these methods require an explicit transformation of the state, whose stability is to be determined, to a fixed point. For a rigidly rotating spiral wave, this can be done by switching to the co-rotating frame of reference, but even finding the steady state in this frame of reference can be a challenging task due to the non-linearity of the problem [108]. Thanks to these studies, it is now known that there are specific meandering and alternans modes which can destabilize spiral waves.

A method from non-linear dynamics which can assess the stability of arbitrary attractors to infinitesimal perturbations is Lyapunov stability analysis. The original idea of a set of characteristic numbers, so-called Lyapunov exponents, quantifying the stability of an attractor of a dynamical system was introduced by A. LYAPUNOV in his doctoral thesis published in 1892 [110]. Lyapunov exponents yield information on the stability of a trajectory in different directions of phase space and can be used to perform bifurcation analysis. A rigorous mathematical theory [111] and practical algorithms for their computation [112, 113] were developed in the second half of the twentieth century, the latter of 
which were mainly targeted at low-dimensional systems of ordinary differential equations (ODEs), but their application to systems with many degrees of freedom is only limited by computational power. Lyapunov stability analysis has recently regained some degree of popularity, due to new algorithms which allow the computation of so-called covariant Lyapunov vectors [114, 115, 116, 117, 118, 119], which are, however, not the subject of this thesis (see section 2.1.8). The very few studies, which have applied Lyapunov stability analysis to excitable media so far, have used it either as a simple indicator for chaos [120], or as a means to compute an effective dimension during chaotic activity [121. To the best of knowledge, there have been no attempts to extract detailed information from the structure of the spectrum of Lyapunov exponents itself. Since it is the first systematic study of Lyapunov spectra of generic excitation patterns in excitable media, the purpose of section 3.1 is twofold: Firstly, the results will be linked to what is already known for low-dimensional systems and analogies (e.g., to coupled oscillator systems) will be given to explain observed Lyapunov exponent spectra for plane waves and single spiral waves. Secondly, the properties of the Lyapunov spectra determined in the first part shall be used to characterize more complex scenarios involving multiple spirals and heterogeneous media. It will be shown that heterogeneities can have ambivalent effects on the complexity of spiral wave or chaotic activity.

In section 3.2, the interaction of electric fields with boundaries of cardiac tissue is investigated. As indicated in section 1.6, a number of studies have aimed at determining the mechanisms that cause bulk depolarization during electric-field stimulation and thus lead to the success of conventional defibrillation. The heterogeneities which were considered include changes in fiber direction with respect to the electric field [122, 123, 97, intercellular clefts due to the microstructure of cardiac tissue [124], random variation of geometrical cell properties [93, 94] and the global geometry of the heart and tissue-bath interface conditions [125, 126]. The strongest kind of heterogeneity in electrical conductance leading to the formation of secondary sources is the tissue boundary itself, since it represents a sudden intracellular conductivity drop to zero. In all of the above studies, it is therefore usually implied that strong effects of electric fields are seen at the tissue boundaries in any case, because they focused at high field strengths. At low field strengths, however, the geometry of those boundaries might influence their susceptibility to electric fields. The importance of knowing where exactly electric fields excite the tissue has been demonstrated in a number of studies which showed that waves pinned to circular non-conducting inclusions in the tissue (i.e. internal tissue boundaries) can be unpinned with electric fields well below the defibrillation threshold, because excitation is induced exactly at those obstacles and therefore close to the spiral center [127, 128, 129, 130, 131, 132, 133. In order to develop low-energy electric-field based antiarrhythmic strategies, it is therefore essential to understand the interaction of electric fields with the tissue substrate and, more specifically, the mechanisms that determine the location of electric-field induced wave emission at low field strengths instead of those well above the defibrillation threshold. Here, the effect of boundary curvature on the depolarization patterns in response to electric fields shall be studied. As a special case, this includes the above-mentioned non-conducting inclusions that present obstacles to the electrical coupling between cardiomyocytes. As seen in figure 1.6, the outer boundaries of the tissue are an additional source of structural 
heterogeneity. As shall be shown in section 3.2, this geometric fine structure gives rise to a variety of electric-field induced effects not observed for simple, large scale geometries. These effects will be characterized utilizing a theory devoloped by A. PUMIR and V. KRINSKY [127] for circular inclusions, which is extended and generalized to arbitrary shapes. The theory is based on a modified boundary condition for the cardiac equations, which implements the effect of an electric field. A numerical technique known as the phase-field method [134] will be extended in section 2.1.5 to be able to apply this modified boundary condition in numerical simulations of arbitrary tissue geometries. Subsequently, using different levels of approximation to the mathematical model, several prototypical shapes of tissue boundaries will be examined for their susceptibility to electric-field stimulation both analytically and numerically.

The subject of section 3.3 is to understand the physiological basis of a recentlydeveloped low-energy defibrillation technique. As indicated in section 1.5 , today, the term defibrillation is inseparably tied to a high-energy electric shock. In the early times of this anti-arrhythmic therapy, pulse shape optimizations (from an alternating current wave form to a biphasic pulse) led to a considerable reduction of the energy required to restore normal sinus rhythm in a fibrillating heart. Reducing the required energy also diminished the risk of potential damage to the myocardium. However, during the last 20 years, the energy could only marginally be lowered, showing that the optimization potential of conventional single-shock defibrillation has been almost exhausted.

However, the paradigm of an absolute defibrillation threshold has recently started to crumble. In 2009, F. Fenton, S. Luther et al. demonstrated in-vitro that atrial fibrillation can be terminated using a series of low-energy shocks instead of one high-energy shock [135], achieving an energy reduction of more than 85\%. Similar energy reduction was observed by W. Li et al. and C. M. AmBrosi et al. using a multi-stage atrial defibrillation technique [136, 137]. For the study by Fenton and Luther, the effectiveness of the method was attributed to the recruitment of many wave sources throughout the tissue and the progressive activation of more and more volume with subsequent waves emitted from these sources (with a stimulation period on the time scale of the arrhythmia). Hence, the image of defibrillation as an all-or-nothing process has to be abandoned, replacing it by the view that field strengths below the defibrillation threshold are still able to induce waves in the muscle, but cannot recruit enough tissue volume to end all irregular activity with a single stimulus. From the viewpoint of control theory, it seems reasonable that spatio-temporal chaos underlying fibrillation can be controlled from a large enough number of control sites. This is supported by the finding by M. Allessie et al. that rapid pacing from one location can control a certain region during fibrillation [138]. Like above for the second topic, knowing the locations of tissue activation can help to assess the limitations and improve the strategy of low-energy anti-fibrillation pacing (LEAP). In view of the generally accepted fact that virtual electrodes in the tissue are mediated by tissue heterogeneity, it is therefore necessary to find out, what kind of heterogeneity provides the substrate for the necessary wave source recruitment in the field strength range covered by LEAP. Based on anatomical data from $\mu \mathrm{CT}$ measurements and the theory of wave source recruitment due to PUMIR and KRINSKY [127], section 3.3 provides experimental evidence for a dominating role of cardiovascular tree as the heterogeneity in question. 


\section{Chapter 2}

\section{Methods}

\subsection{Mathematical background}

An important class of results generated by biophysical research in the twentieth century is that of mathematical models, both of complete biological systems and of their constituting parts. A major breakthrough was the mathematical description of cell electrophysiology, beginning with the modeling of action potential propagation in the giant squid axon (a large nerve fiber) by HoDGKIN and HuXley. In fact, their paper [139] is a summary of a series of preceding publications by HodgKin, Huxley and KATZ [140, 141, 142, 143]. At the time, its novelty lay in the fact that the authors not only systematically studied transmembrane currents of different ion species using the newly established method of voltage clamp [144, 145]. They also gathered enough data on the voltage-determined, ion-specific permeability $\left.\right|^{1}$ of the membrane to combine all of their findings in a mathematical framework that accurately reproduces the experimental action potentials, including propagation along the nerve fiber.

Their results have essentially remained valid despite the progress that has been made in the discovery of molecular mechanisms leading to the observed ion current dynamics. This fact emphasizes the broad range of applicability of their method which is independent of the internal functioning of the biological substrate and thus rootedly empirical. D. NoBLE was one of the first to apply the model to cardiac excitable tissue, such as Purkinje fibers [146, 147. All modern schemes of building electrophysiological models of excitable cells such as neurons, cardiomyocytes or muscle cells are still based on this method, merely using improved and more detailed experimental tools for identifying the active membrane proteins such as the patch clamp technique [148] and expression systems for ion channels [149]. Such models are therefore called Hodgkin-Huxley type models.

In the following sections, the necessary theoretical background for modeling cardiac tissue (and excitable media in general) will be introduced. They form the basis of all numerical work in this thesis. Following this fundamental theoretical framework, the specific models and analysis methods utilized in this work will be presented. In addition to the above-mentioned electrophysiology, a full mathematical description of cardiac tissue (up to the state of current knowledge) would have to cover many more aspects than just the pure electrophysiology, the most evident of them being excitation-contraction coupling -

\footnotetext{
${ }^{1}$ The molecular basis of this variable permeability of the membrane (ion channels, cf. section 1.2 was not known at the time.
} 


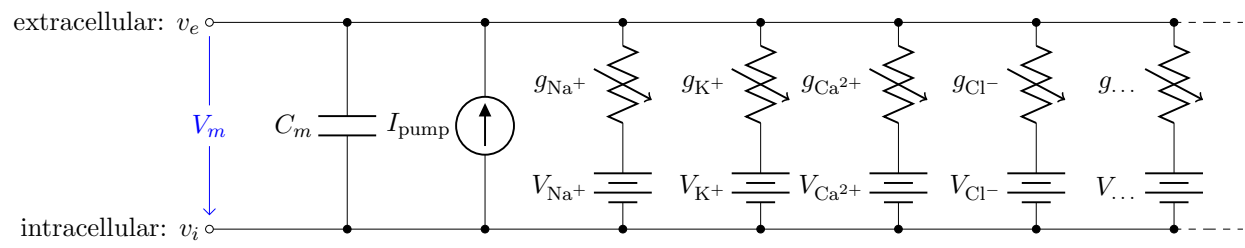

Figure 2.1: Equivalent circuit diagram for the cell membrane. The cell membrane is modeled as a capacitor that is charged to the membrane voltage $V_{m}$ by the Nernst potentials $\left(V_{\mathrm{Na}^{+}}, \ldots\right)$ of different ion species. Variable resistors indicate varying individual permeability of the membrane for each ion species. The current source corresponds to ion transporters in the membrane necessary for ion homeostasis.

indispensable for the normal physiological function. However, using Occam's razor, the presented methods will be restricted to those aspects that are necessary to understand the results in section 3. It should also be noted that, despite the general agreement on the nature of action potentials in excitable cells and the huge system of theories that is based on the pioneering work by HoDGKIN and HuXLEY, there is also a recent alternative explanation of propagating nerve impulses based on soliton waves in traveling in the cell membrane [150, 151, 152], which is, however, highly controversial and shall not be elaborated further upon in this work.

\subsubsection{Single cell dynamics}

Following HodgKin's and HuXLEys's scheme, the electrical behavior of the cell membrane can be modeled by an equivalent circuit as shown in figure 2.1: The (impermeable part of the) cell membrane is represented by a capacitor with capacity $C_{m}$. It can be charged by the accumulation of charge on its two plates (the inner and outer surfaces of the cell membrane) and the potential difference $V_{m}=v_{i}-v_{e}$ between the intracellular and the extracellular potentials is called membrane potential, as explained in section 1.2.1. This happens through passive and active ion channels that determine the permeability and thus the electrical conductivity of the membrane for specific ionic species $\left(g_{\mathrm{x}}\right.$ for ion species $\left.\mathrm{x}\right)$. Denoting the current through the membrane of ion species x by $I_{\mathrm{x}}$ and the charge on the capacitor by $Q$, Kirchhoff's current law implies that the sum of all currents (through the vertical elements in figure 2.1) is zero

$$
\begin{aligned}
0 & =\frac{\mathrm{d} Q}{\mathrm{~d} t}+I_{\mathrm{pump}}+I_{\mathrm{Na}^{+}}+I_{\mathrm{K}^{+}}+I_{\mathrm{Ca}^{2+}}+I_{\mathrm{Cl}^{-}}+I_{\ldots} \\
\Rightarrow \quad \frac{\mathrm{d} Q}{\mathrm{~d} t} & =C_{m} \frac{\mathrm{d} V_{m}}{\mathrm{~d} t}=-I_{\mathrm{pump}}-I_{\mathrm{Na}^{+}}-I_{\mathrm{K}^{+}}-I_{\mathrm{Ca}^{2+}}-I_{\mathrm{Cl}^{-}}-I_{\ldots}
\end{aligned}
$$

resulting in the above equation for the membrane potential $V_{m}$. In the above equation, the convention was used that positive currents point out of the cell, i.e. from intracellular to extracellular, which implies that the charge $Q$ is the one on the intracellular "plate" of the capacitor. The right hand side is sometimes summarized in a term $I_{\text {ion }}$. Each term $I_{\mathrm{x}}$, according to the circuit diagram in figure 2.1 is of the form

$$
I_{\mathrm{x}}=g_{\mathrm{x}}\left(V_{m}-V_{\mathrm{x}}\right),
$$


where $V_{\mathrm{x}}$ is the reversal potential of the ion species $\mathrm{x}$. The dots in equation (2.2) and figure 2.1 represent additional ion species and dynamic elements that could be included in the model. Due to the existence of multiple ion channel types for the same ionic species, the currents associated with each species in figure 2.1 have to be interpreted as net currents and $g_{\mathrm{x}}$ as a net (average) conductivity. Thus, the model could be refined by treating each ion channel type as a separate circuit element. This is indeed done in physiologically detailed models such as the Wang-Sobie model [153], but it is not necessary for the purpose of this thesis.

In contrast, an important aspect that has to be taken into account and that is at the basis of the nonlinear phenomenon of action potential formation is the variability of the conductances $g_{\mathrm{x}}$. These are functions of time as well as the quantity gating the channel (membrane potential, individual ion concentrations or mechanical stress, cf. section 1.2.1 and reference [25]). As mentioned in the introduction of this chapter, the development of the Hodgkin-Huxley formalism preceded the discovery of the molecular functioning of ion channels. Still, from the measured current traces in voltage clamp experiments, it was already clear that the dynamics of the individual ion conductances can themselves only be modeled by dynamical systems for the so-called gating variables. These describe the transitions between different states of the ion channel or its subunits, the main parameters being the transition rates that are, in turn, determined by the quantity responsible for gating the channel (e.g., $V_{m}$ for voltage-gated channels). Depending on the absolute number of channels, a stochastic or deterministic model for the gating variables is required to accurately model the overall membrane conductance for a particular ionic species [154, 155. As this thesis deals with macroscopic systems only, deterministic dynamics modeled by differential equations will be assumed in the following. In general, each current term in equation $(2.2)$ is thus supplemented by a number of additional ordinary differential equations describing its (in-)activation dynamics. The full system of equations describing a single cell electrophysiological model in its most abstract form thus reads

$$
\begin{aligned}
C_{m} \frac{\mathrm{d} V_{m}}{\mathrm{~d} t} & =\overbrace{-I_{\text {pump }}\left(V_{m}, \mathbf{h}\right)-\sum_{\mathrm{x}} I_{\mathrm{x}}\left(V_{m}, \mathbf{h}\right)}^{-I_{\text {ion }}\left(V_{m}, \mathbf{h}\right)} \\
\frac{\mathrm{d} \mathbf{h}}{\mathrm{d} t} & =\mathbf{H}\left(V_{m}, \mathbf{h}\right),
\end{aligned}
$$

where the vector $\mathbf{h}$ consists of the gating and all further variables necessary to model the individual components (this may also include ion concentrations, etc). All current electrophysiological cell models have the form of equation (2.4) and the number of terms on the right hand side varies with model complexity, either absorbing many summands into one term or splitting some of them up into individual contributions (see section 2.1.6 for details).

\subsubsection{Bi-domain description of cardiac tissue}

The single-cell model introduced in section 2.1.1 can be thought of as a description for an average piece of the cell membrane without any spatial extent. In this case, $C_{m}, Q$ and the current $I_{\text {ion }}$ in equation (2.4) have to be interpreted as capacitance, charge and 
current per unit membrane area. This can be used to construct a model of a $d$-dimensional domain of cardiac tissue by extending the so-called core conductor model of neuronal cable theory [156, an idea that emerged during efforts in the 1970s to model surface electrocardiograms [157, 158, 159]. The resulting mathematical framework is currently viewed as the standard and most accurate model of cardiac tissue as a continuum [160, 161, 32. If an extended slab of cardiac tissue is considered, the intracellular potentials, $v_{i}(\mathbf{x}, t)$ and $v_{e}(\mathbf{x}, t)$, respectively, become functions of the position $\mathbf{x}$ in space, i.e. $\mathbf{x} \in \mathbb{R}^{d}, t \in \mathbb{R}$. The idea behind this is that both intracellular and extracellular spaces are defined in the whole tissue domain (thus occupying the same physical space) and have conductivity tensors $\underline{\sigma}_{i}$ and $\underline{\sigma}_{e}$, respectively, that are averaged quantities over multiple cells. The relative volumes of the physical intracellular and extracellular spaces and the geometrical arrangement of cells are implicitly contained in these averaged conductivities, as is the additional resistance introduced by gap junctions in the intracellular domain. The two domains represent spatially extended versions of the capacitor plates considered in the single cell model. Current densities within each domain caused by the corresponding potentials are:

$$
\begin{aligned}
& \mathbf{j}_{i}=-\underline{\sigma}_{i} \nabla v_{i} \\
& \mathbf{j}_{e}=-\underline{\sigma}_{e} \nabla v_{e}
\end{aligned}
$$

The current densities enter continuity equations for the charge densities $q_{i}$ and $q_{e}$ in the intracellular and extracellular domain, respectively. It is assumed that no charge accumulates anywhere in the tissue, such that $\partial q_{i} / \partial t=-\partial q_{e} / \partial t=: \partial q / \partial t$ and

$$
\begin{aligned}
& \nabla \cdot \mathbf{j}_{i}+\frac{\partial q}{\partial t}=-i_{\text {ion }} \\
& \nabla \cdot \mathbf{j}_{e}-\frac{\partial q}{\partial t}=i_{\text {ion }}
\end{aligned}
$$

where the lowercase quantities $q$ and $i_{\text {ion }}$ are charge and membrane current per unit volume, respectively. Otherwise, the same sign conventions as in section 2.1.1 apply. The membrane currents appear as sources and sinks for the extracellular and intracellular domain, respectively, as crossing the cell membrane is the only way for charge to leave one of the two domains (in the absence of current injection via electrodes). As the corresponding quantities $Q$ and $I_{\text {ion }}$ are given per unit membrane area, these quantities have to be converted using the surface-to-volume ratio $\beta$ (units: area per volume) which specifies how much membrane surface there is on average for a given volume of tissue. Combining equations (2.5) and (2.6), the bi-domain equations for the tissue domain $\mathcal{D}$ are obtained:

$$
\left.\begin{array}{rl}
\nabla \cdot \underline{\sigma}_{i} \nabla v_{i} & =-\nabla \cdot \mathbf{j}_{i}=\beta\left(\frac{\partial Q}{\partial t}+I_{\text {ion }}\right)=\beta\left(C_{m} \frac{\partial\left(v_{i}-v_{e}\right)}{\partial t}+I_{\text {ion }}\right) \\
\nabla \cdot \underline{\sigma}_{e} \nabla v_{e} & =-\nabla \cdot \mathbf{j}_{e}=-\beta\left(\frac{\partial Q}{\partial t}+I_{\text {ion }}\right)=-\beta\left(C_{m} \frac{\partial\left(v_{i}-v_{e}\right)}{\partial t}+I_{\text {ion }}\right)
\end{array}\right\} \text { in } \mathcal{D}
$$

These equations have to be completed by boundary conditions for $v_{i}$ and $v_{e}$ at the boundary $\partial \mathcal{D}$ of the tissue domain $\mathcal{D}$. Assuming the space outside the tissue domain $\mathcal{D}$ is a mono-domain with conductivity $\underline{\sigma}_{o}$ and a corresponding outside potential $v_{o}$, these bound- 
ary conditions are

$$
\left.\begin{array}{rl}
\mathbf{n} \cdot \underline{\sigma}_{i} \nabla v_{i} & =0 \\
v_{e} & =v_{o} \\
\mathbf{n} \cdot \underline{\sigma}_{e} \nabla v_{e} & =\mathbf{n} \cdot \underline{\sigma}_{o} \nabla v_{o}
\end{array}\right\} \quad \text { on } \partial \mathcal{D}
$$

where $\mathbf{n}$ is a local unit vector perpendicular to the boundary $\partial \mathcal{D}$. Equation $2.8 \mathrm{a}$ is a no-flux boundary condition, expressing the fact that the intracellular space ends at the tissue boundary and thus no current in the intracellular domain can cross this boundary. Equation 2.8b and equation 2.8c ensure the continuity of the extracellular potential and currents at the interface between the extracellular space inside the tissue and the mono-domain outside the tissue (e.g., the bath).

For practical calculations, a different linear combination of the system 2.7) is used which corresponds to a change of variables from $v_{i}$ and $v_{e}$ to $V_{m}=v_{i}-v_{e}$ and $v_{e}$ :

$$
\left.\begin{array}{l}
\nabla \cdot \underline{\sigma}_{i} \nabla(\overbrace{V_{m}+v_{e}}^{v_{i}}=\beta\left(C_{m} \frac{\partial V_{m}}{\partial t}+I_{\text {ion }}\right) \\
\nabla \cdot \underline{\sigma}_{e} \nabla v_{e} \stackrel{2.7 a}{=} \stackrel{+2.7 b}{=}-\nabla \cdot \underline{\sigma}_{i} \nabla v_{i}=-\nabla \cdot \underline{\sigma}_{i} \nabla\left(V_{m}+v_{e}\right)
\end{array}\right\} \quad \text { in } \mathcal{D}
$$

Reordering the terms in both equations yields

$$
\left.\begin{array}{ll}
C_{m} \frac{\partial V_{m}}{\partial t} & =\frac{1}{\beta} \nabla \cdot \underline{\sigma}_{i} \nabla\left(V_{m}+v_{e}\right)-I_{\text {ion }}\left(V_{m}, \mathbf{h}\right) \\
\frac{\partial \mathbf{h}}{\partial t} & =\mathbf{H}\left(V_{m}, \mathbf{h}\right) \\
\nabla \cdot\left(\underline{\sigma}_{e}+\underline{\sigma}_{i}\right) \nabla v_{e} & =-\nabla \cdot \underline{\sigma}_{i} \nabla V_{m} .
\end{array}\right\} \text { in } \mathcal{D}
$$

Having been omitted so far, equation (2.9b) was reinserted from equation (2.4) for completeness. Equation $2.9 \mathrm{c}$ does not contain $t$ and thus states that the extracellular potential can be determined from the membrane potential at any given instant. In equation 2.9a), the term $\nabla \cdot \underline{\sigma}_{i} \nabla\left(V_{m}+v_{e}\right)$ is more favorable than the seemingly simpler $-\nabla \cdot \underline{\sigma}_{e} \nabla v_{e}$, because in this way, the equation stays valid in the limit $\underline{\sigma}_{e}=\alpha \underline{\sigma}_{e}^{*}$ for $\alpha \rightarrow \infty$. This will be useful in the next section. For the boundary conditions (2.8), the change of variables can be achieved by replacing $V_{m}$ in equation 2.8a):

$$
\left.\begin{array}{rl}
\mathbf{n} \cdot \underline{\sigma}_{i} \nabla\left(V_{m}+v_{e}\right) & =0 \\
v_{e} & =v_{o} \\
\mathbf{n} \cdot \underline{\sigma}_{e} \nabla v_{e} & =\mathbf{n} \cdot \underline{\sigma}_{o} \nabla v_{o}
\end{array}\right\} \quad \text { on } \partial \mathcal{D}
$$

\subsubsection{Mono-domain descriptions of cardiac tissue}

For a full numerical simulations using the bi-domain equations 2.9 , one would have to time-step the membrane potential $V_{m}$ using equation 2.9a from a given present state and calculate the corresponding extracellular potential by solving equation $2.9 \mathrm{c}$ for $v_{e}$. The latter requires solving a large system of linear equations, an operation that is computationally very costly. Because of this, there exists an approximation that only involves a single partial differential equation (PDE) for $V_{m}$ that can be solved by time stepping, the 
so-called mono-domain equation. Although the mono-domain model is used extensively in the literature and is regarded a valid model in its own right without further justification, it is important to know its connection to the full and more realistic bi-domain formulation. In particular, this is true for understanding the effects of electric fields near tissue boundaries, which is one of the aims of this thesis. In the following two sub-sections, two different ways of deriving the mono-domain equation with different physical interpretation will be presented. When working with a mono-domain equation, one has to ensure that modifications to the equations are consistent with the choice of interpretation.

\section{Neglecting the extracellular potential}

Assume a very well-conducting extracellular space 2 i.e. $\alpha \gg 1$ for $\underline{\sigma}_{e}=\alpha \underline{\sigma}_{e}^{*}$. In the limit $\alpha \rightarrow \infty$, equation (2.9c) reduces to $\nabla \cdot \underline{\sigma}_{e}^{*} \nabla v_{e}=0$. This means that for large $\alpha$ the source term for the extracellular potential becomes negligible and one possible form of solution is $v_{e}=-\mathbf{E} \cdot \mathbf{x}+c$ with some fixed electric field vector $\mathbf{E}$ and an arbitrary constant $c$ (if boundary conditions 2.10b and 2.10c permit) ${ }^{3}$. For this solution, it has to be assumed that $\underline{\sigma}_{e}^{*}$ is spatially constant. If we assume the extracellular space is insulated $\left(\mathbf{n} \cdot \sigma_{e}^{*} \nabla v_{e}=0\right)$ or grounded $\left(v_{e}=0\right)$ on $\partial \mathcal{D}$, this solution reduces even further to $v_{e}=c$ or $v_{e} \equiv 0$, respectively. In contrast, equation $2.9 \mathrm{a}$ is still valid. The mono-domain equation in this scenario therefore is

$$
\frac{\partial V_{m}}{\partial t}=\nabla \cdot \underline{\mathbf{D}} \nabla\left(V_{m}+v_{e}\right)-I_{\text {ion }} / C_{m} \text { in } \mathcal{D},
$$

where the diffusion tensor is given by $\underline{\mathbf{D}}=\underline{\sigma}_{i} /\left(\beta C_{m}\right)$ and $v_{e}$ is merely a parameter for the problem, which is usually set to zero following the line of thoughts above. To make this problem well-defined, the boundary condition 2.10a is sufficient:

$$
\mathbf{n} \cdot \underline{\mathbf{D}} \nabla\left(V_{m}+v_{e}\right)=0 \quad \text { on } \partial \mathcal{D}
$$

\section{Equivalent mono-domain description}

In section 2.1.3, a mono-domain formulation of the bi-domain model was obtained by changing the physics of the problem. The extent, to which the dynamics is altered can be significantly reduced in the case were the intracellular and extracellular conductivity tensors are scaled versions of the same tensor: $\underline{\sigma}_{e}=\alpha \underline{\sigma}_{i}$. In this case, equation (2.7b) can be subtracted from equation 2.7a):

$$
\nabla \cdot \underline{\sigma}_{i} \nabla V_{m}=\left(1+\alpha^{-1}\right) \beta\left(C_{m} \frac{\partial V_{m}}{\partial t}+I_{\text {ion }}\right)
$$

\footnotetext{
${ }^{2}$ Incidentally, this case brings the model of cardiac tissue very close to the neuronal cable equation from which it branched off. There, the assumption of an infinite conductivity in the extracellular space is justified by the large amount of fluid that surrounds the axon.

${ }^{3}$ Other solutions are possible, viz. all those for which a vector potential $\mathbf{A}$ exists with $\underline{\sigma}_{e}^{*} \nabla v_{e}=\nabla \times \mathbf{A}$. For $v_{e}=-\mathbf{E} \cdot \mathbf{x}$, choose $\mathbf{A}=-\left(\left(\underline{\sigma}_{i} \mathbf{E}\right)_{3} x_{2},\left(\underline{\sigma}_{i} \mathbf{E}\right)_{1} x_{3},\left(\underline{\sigma}_{i} \mathbf{E}\right)_{2} x_{1}\right)^{T}$
} 
Rearranging for $\partial V_{m} / \partial t$ yields the mono-domain equation

$$
\left.\begin{array}{rl}
\frac{\partial V_{m}}{\partial t} & =\nabla \cdot \underline{\mathbf{D}} \nabla V_{m}-I_{\text {ion }}\left(V_{m}, \mathbf{h}\right) / C_{m} \\
\frac{\partial \mathbf{h}}{\partial t} & =\mathbf{H}\left(V_{m}, \mathbf{h}\right)
\end{array}\right\} \quad \text { in } \mathcal{D}
$$

where now $\underline{\mathbf{D}}=\underline{\sigma}_{i} /\left[\beta C_{m}\left(1+\alpha^{-1}\right)\right]$. In the limit $\alpha \rightarrow \infty$, the definition of $\underline{\mathbf{D}}$ is equal to that in equation (2.11). Similarly, the $v_{e}$ term in equation (2.11) becomes irrelevant if $\underline{\sigma}_{e}^{*}$ is a scaled $\underline{\sigma}_{i}$, because then $\nabla \cdot \underline{\mathbf{D}} \nabla v_{e}=0$ follows from $\nabla \cdot \underline{\sigma}_{e}^{*} \nabla v_{e}=0$. Equation 2.11) and equation (2.13) are thus consistent in the sense that they are equivalent when the assumptions for both mono-domain descriptions are fulfilled.

Note that the mono-domain description 2.13 is completely equivalent to the full bidomain model, but numerically cheap compared to equation (2.9). Consequently, for the case $\underline{\sigma}_{e}=\alpha \underline{\sigma}_{i}$, numerical simulation of the bi-domain model is usually superfluous. There is, however, one caveat: from the set of boundary conditions 2.10$)$, no boundary condition for the membrane potential alone can be derived. Also, unlike in equation (2.11), $v_{e}$ is not decoupled from the membrane potential dynamics and so equation 2.10a cannot be enforced unless $v_{e}$ is calculated, which would defeat the original purpose of a mono-domain formulation. The usual (somewhat nonphysical) solution to this dilemma is to apply the ad-hoc no-flux boundary condition

$$
\mathbf{n} \cdot \underline{\mathbf{D}} \nabla V_{m}=0 \quad \text { on } \partial \mathcal{D} .
$$

This is sufficient for modeling the dynamics inside a tissue domain $\mathcal{D}$, where the boundary of the domain is not meant to be a physical part of the system but rather a computational necessity. However, it is only a valid simplification, if one can rule out any influence of the boundary condition on the effects one wants to study. Sometimes, the no-flux boundary condition is physically justified, for example, if the extracellular space is known to be insulated electrically at the tissue boundary. However, in particular in this thesis, investigating effects at the boundaries of the tissue due to the conductive connection of the extracellular space to the surrounding environment is the original purpose of the modeling. In this case, an alternative solution is to assume that $\alpha$ and the outside conductivity $\underline{\sigma}_{o}$ are large, which decouples the corresponding potentials from the dynamics of the membrane potential and makes them parameters as seen for equation (2.11). This way, an extracellular or outside potential (e.g., a uniform electric field) can be assumed rather than calculated, which makes it possible to use equation 2.10a itself or a boundary condition obtained by subtracting equation 2.10c from equation 2.10a):

$$
\begin{aligned}
& \mathbf{n} \cdot \underline{\mathbf{D}} \nabla\left(V_{m}+v_{e}\right)=0 \quad \text { on } \partial \mathcal{D} \\
& \text { or } \\
& \mathbf{n} \cdot \underbrace{\alpha \underline{\sigma}_{i}}_{=\underline{\sigma}_{e}} \nabla V_{m}=-\mathbf{n} \cdot \underline{\sigma}_{o} \nabla v_{o} \quad \text { on } \partial \mathcal{D}
\end{aligned}
$$

The second boundary condition 2.16 will be used in section 3.2 to study the effect of electric fields near boundaries of the tissue. As for the bi-domain model (2.9), the mono- 
domain equation (2.13) has to be supplemented by a concrete model $\mathbf{H}$ for the local variables $\mathbf{h}$ and the corresponding ionic currents $I_{\text {ion }}\left(V_{m}, \mathbf{h}\right)$. The models used in this thesis will be introduced in section 2.1.6.

\subsubsection{Anisotropy}

Although all simulations in this thesis are carried out assuming isotropic diffusion, for completeness of the mathematical framework, the construction of the corresponding tensors for the specific requirements of cardiac tissue shall be mentioned here briefly: The anisotropic conductivity of cardiac tissue due to the fiber orientation and sheet structure of the muscle (see section 1.3 can be implemented in the bi-domain and in the monodomain model by choosing the diffusion tensor $\underline{\mathbf{D}}$ or equivalently the conductivity tensors. D can be constructed from its three eigenvectors, each of which represents a direction in which a potential gradient leads to a current density in the same direction, with a conductivity equal to the corresponding eigenvalue. The fiber orientation determines one of these directions, the other two are uniquely defined by the normal vector of the laminar sheets (the remaining vector thus corresponds to the direction perpendicular to the fibers within the sheet). These three eigenvectors form an orthonormal basis of $\mathbb{R}^{3}$, implying that $\underline{\mathbf{D}}$ must be a real, symmetric matrix. Using these properties, the entire tensor $\underline{\mathbf{D}}$ is determined by two unit vectors in space defining the fiber direction $\mathbf{f}=\left(f_{x}, f_{y}, f_{z}\right)$ and the direction normal to the sheet plane $\mathbf{n}=\left(n_{x}, n_{y}, n_{z}\right)$ and by diffusion constants $D_{\|}$ along the fibers, $D_{\perp}$ perpendicular to the fibers (within the sheet) and $D_{\perp \perp}$ normal to the sheets (see equation 17 in [32]):

$$
D_{i j}=\delta_{i j} D_{\perp}+\left(D_{\|}-D_{\perp}\right) f_{i} f_{j}+\left(D_{\perp \perp}-D_{\perp}\right) n_{i} n_{j}, \quad i, j \in\{x, y, z\}
$$

A common simplification is to ignore the sheet structure and thus assume $D_{\perp \perp}=D_{\perp}$, which eliminates one of the terms in equation (2.17). Note also that, in general, both the directions and diffusion constants may vary spatially.

The so-called anisotropy ratio is defined as the quotient $D_{\|} / D_{\perp}$. In the bi-domain equations, the two conductivity tensors $\underline{\sigma}_{e}$ and $\underline{\sigma}_{i}$ can be chosen independently. While the fiber orientation for both is the same, because it is given by the geometrical arrangement of the cells, the anisotropy ratio can be different. The case of equal anisotropy ratio is the one considered in the mono-domain approximation of section 2.1.3.

\subsubsection{The phase-field method}

As outlined in section 2.1.3, boundary conditions constitute an important part of any problem described by a PDE on a spatial domain $\mathcal{D}$. It was mentioned that a widely-used boundary condition for mono-domain cardiac models is no-flux. Another possibility which was derived for the case of a predetermined extracellular/outside potential is that of equation 2.16 in the same section. Both boundary conditions are of the Neumann type, i.e. they fix the normal fluxes $\mathbf{n} \cdot \underline{\mathbf{D}} \nabla u$ on the boundary $\partial \mathcal{D}$. As will be shown in section 2.2.3. for a finite-difference numerical scheme, no-flux boundary conditions are straightforward to implement, if the boundaries of the domain $\mathcal{D}$ are parallel to the coordinate axes, e.g., rectangles in cartesian coordinates or (fractional) annuli in polar coordinates. For ar- 
bitrarily shaped domains $\mathcal{D}$, there is no such natural way of imposing zero flux on the domain boundary. In this case, one usually resorts to more complex and computationally intensive numerical schemes like finite-volume or finite-element methods (see section 6.3 of reference [32] and references therein).

In reference [134, FenTon et al. introduce a phase-field method to impose no-flux boundary conditions on arbitrary geometries using finite differences on a simple (e.g., rectangular) computational domain $\mathcal{D}_{c}$ which contains the actual physical domain $\mathcal{D}$. In the following, this method will be explained in detail and extended to arbitrary Neumann boundary conditions of the type of equation (2.16). Furthermore, mathematical improvements to the convergence proof will enable a better assessment of the method's numerical accuracy. The generalized method will be used extensively in section 3.2 to study the effect of electric fields at boundaries of curved tissue domains.

\section{Description of the method}

The phase-field method is based on defining a phase field $\phi$, which is a smoothed version of the characteristic function of $\mathcal{D}$, i.e. $\phi \approx 1$ inside the domain $\mathcal{D} \subset \mathcal{D}_{c}$ and $\phi \approx 0$ otherwise, with a smooth transition in between. In this thesis, a different dynamical system than suggested in reference [134] shall be used for obtaining $\phi$ from the sharp, original characteristic function $\phi_{0}$ :

$$
\frac{\partial \phi}{\partial t}=\left(\phi_{0}-\phi\right)+\xi^{2} \Delta \phi
$$

In this equation, $\phi$ approaches a smooth phase field for $t \rightarrow \infty$, which stays close to $\phi_{0}$ and has a diffusive boundary with a characteristic length scale $\xi$ of the resulting transition at the interface between $\phi=1$ and $\phi=0$. It does not suffer from the stability issues in higher dimensions mentioned in [134] for the double-well potential method defined therein. Hence, any method that finds the equilibrium of equation 2.18 may be used instead of explicit time-stepping. In the following, this steady-state solution will be referred to as $\phi$. An example for $\phi_{0}$ and the resulting $\phi$ is depicted in figures 2.2 a) and b), respectively. One property that will prove to be important for the line of thoughts below is that the normal slope of $\ln \phi$ decreases rapidly and monotonically within the interface region (in the outward direction) until reaching an approximately constant value on the order of $-1 / \xi$, as shown in figure 2.2 ). Hence, at large normal distances from the interface, $\phi$ decays approximately exponentially and $\partial^{2} \ln \phi / \partial n^{2} \approx 0$. As illustrated in figure $2.2 \mathrm{~d}$ ), the normal direction is defined by the direction of the gradient $\nabla \ln \phi$ itself.

The phase field method then consists of substituting the original diffusion term (e.g., in equation (2.13) ) by a modified term that involves the phase field $\phi$ :

$$
\nabla \cdot(\underline{\mathbf{D}} \nabla u) \quad \rightarrow \quad \frac{1}{\phi} \nabla \cdot(\phi \underline{\mathbf{D}} \nabla u)
$$

The resulting PDE after the substitution is assumed to be defined on the whole domain $\mathcal{D}_{c}$, but only the part of the solution in $\mathcal{D} \subset \mathcal{D}_{c}$ is taken to have physical meaning. Fenton et al. present a mathematical argument in reference [134] for a one-dimensional tissue domain which suggests that the substitution 2.19 for $\mathcal{D}_{c}$, leads to a solution in $\mathcal{D}$ that 
a)

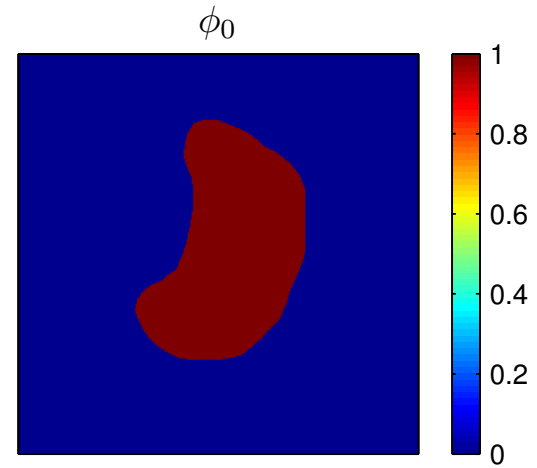

c)

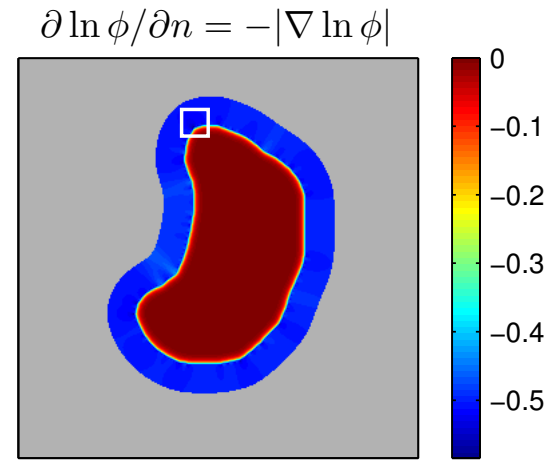

b)

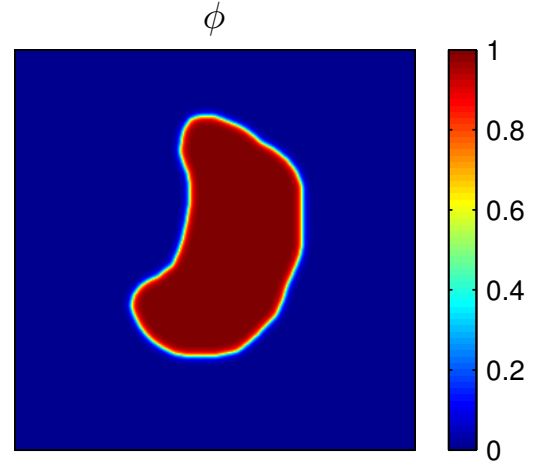

d)

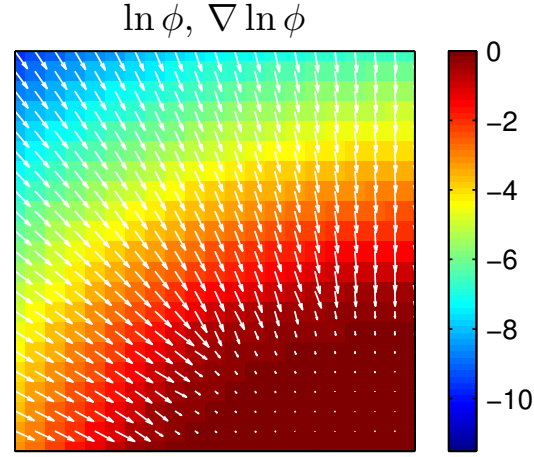

Figure 2.2: Phase field behavior. a) Initial binary phase field $\phi_{0}$, i.e. the characteristic function of $\mathcal{D}$. b) Steady-state solution of equation (2.18) with $\phi_{0}$ from a) and $\xi=2$. c) $|\nabla \ln \phi|$ which is equal to $-\partial \ln \phi / \partial n$ if one defines the normal vector $\mathbf{n}$ to be antiparallel to $\nabla \ln \phi$. The logarithmic normal derivative increases quickly from 0 inside $\mathcal{D}$ to an approximately constant value $\approx 1 / \xi$ outside $\mathcal{D}$. d) $\ln \phi$ shown as a color plot and the corresponding gradient shown as arrows for the region indicated by a white rectangle in c). 


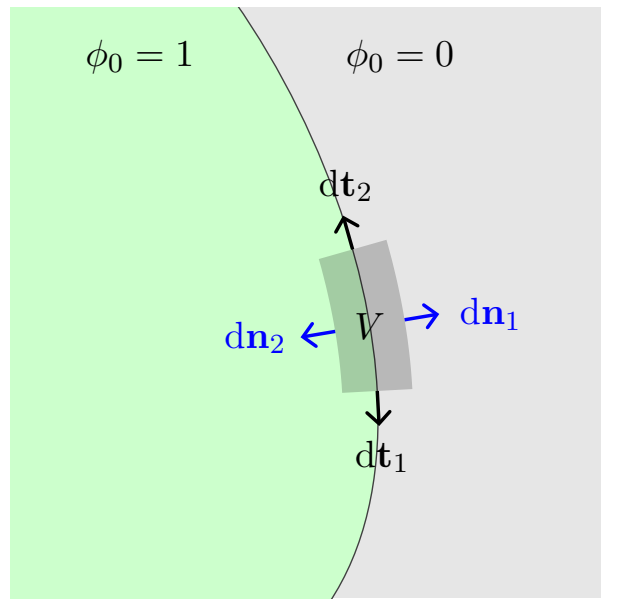

Figure 2.3: Volume integration at the phase-field interface. Sketch of the integration volume for equation (2.22) parallel to the boundary $\partial \mathcal{D}$ which is defined by the border between $\phi_{0}=1$ (green) and $\phi_{0}=0$ (gray). Arrows indicate normal vectors on the surfaces of the integration volume corresponding to the individual terms in equation (2.23).

obeys a no-flux boundary condition

$$
\mathbf{n} \cdot(\underline{\mathbf{D}} \nabla u)=0 \quad \text { on } \partial \mathcal{D} \text {. }
$$

A similar proof for higher dimensions based on the same idea was provided in reference 162. In the following, this idea and its shortcomings in terms of the necessary mathematical assumptions will be presented and an argument will be given how these deficits may be resolved.

\section{Original convergence proof}

Omitting any additional non-spatial degrees of freedom for the reaction term, the general PDE for a reaction-diffusion system with the replacement equation $(2.19)$ is

$$
\frac{\partial u}{\partial t}=f(u)+\frac{1}{\phi} \nabla \cdot(\phi \underline{\mathbf{D}} \nabla u),
$$

where $f$ is assumed to be bounded for bounded $u$. The multi-dimensional equivalent of the mathematical argument given in [134] starts by integrating equation (2.21) over a small volume $V$ across the boundary $\partial D$, which yields

$$
\int_{V} \phi \frac{\partial u}{\partial t} \mathrm{~d} V=\int_{V} \phi f(u) \mathrm{d} V+\int_{V} \nabla \cdot(\phi \underline{\mathbf{D}} \nabla u) \mathrm{d} V=\int_{V} \phi f(u) \mathrm{d} V+\oint_{\partial V} \phi \underline{\mathbf{D}} \nabla u \cdot \mathrm{d} \mathbf{A} .
$$

The geometrical situation is illustrated in figure 2.3 , with the volume $V$ shaded gray. A similar strategy for a different integration path is given in reference [163], but the following argumentation applies universally. Denoting the individual contributions to the surface 
integral separately leads to

$$
\begin{aligned}
\int_{V} \phi \frac{\partial u}{\partial t} \mathrm{~d} V= & \int_{V} \phi f(u) \mathrm{d} V \\
& +\int_{\partial V_{n}^{1}} \phi \underline{\mathbf{D}} \nabla u \cdot \mathrm{d} \mathbf{n}_{1} \\
& +\int_{\partial V_{n}^{2}} \phi \underline{\mathbf{D}} \nabla u \cdot \mathrm{d} \mathbf{n}_{2} \\
& +\int_{\partial V_{t}^{1}} \phi \underline{\mathbf{D}} \nabla u \cdot \mathrm{d} \mathbf{t}_{1} \\
& +\int_{\partial V_{t}^{2}} \phi \underline{\mathbf{D}} \nabla u \cdot \mathrm{d} \mathbf{t}_{2} \\
& +\ldots
\end{aligned}
$$

The dots indicate that, in the case of three dimensions, there are two more terms for another direction parallel to the boundary $\partial \mathcal{D}$. However, for everything presented in the following, these terms can be treated analogously to the two explicitly mentioned in equation 2.23). For a given interface width $\xi$ of $\phi$, the normal width of the volume $V$ is chosen such that the two boundaries of $V$ parallel to the boundary $\partial \mathcal{D}$ are at iso- $\phi$ levels of $\phi=1-\varepsilon$ and $\phi=\varepsilon$ for an arbitrarily small $\varepsilon>0$.

By assuming smooth and bounded solutions $u$ and choosing sufficiently small $V$ (corresponding to small enough $\xi$ for fixed $\varepsilon$ ), the individual integrands of equation 2.23 can be approximated by constants, except for the variation due to the factor $\phi$. By then letting $\xi$ tend to zero, i.e. reducing the width of $V$ normal to the boundary $\partial \mathcal{D}$ and making $\phi$ steeper and steeper, the integral on the left hand side and the integrals of the tangential components $\partial V_{t}^{1}$ and $\partial V_{t}^{2}$ on the right hand side of equation 2.23 vanish, because they are integrated over a volume and a surface, respectively, whose size tends to zero. The same is true for the reaction term, which is assumed to be bounded for bounded $u$. Using the fact that $\phi \approx 1$ on $\partial V_{n}^{2}$ and $\phi \approx 0$ on $\partial V_{n}^{1}$, only one of the two remaining integrals actually has a non-zero contribution, resulting in

$$
\begin{aligned}
0=\int_{\partial V_{n}^{2}} \phi \underline{\mathbf{D}} \nabla u \cdot \mathrm{d} \mathbf{n}_{2} & \approx\left|\partial V_{n}^{2}\right| \underline{\mathbf{D}} \nabla u \cdot \mathbf{n} \\
& \Rightarrow \mathbf{n} \cdot \underline{\mathbf{D}} \nabla u=0,
\end{aligned}
$$

where $\left|\partial V_{n}^{2}\right|$ denotes the surface area of $\partial V_{n}^{2}$.

If the above reasoning was justified, equation 2.25) would indicate that no-flux boundary conditions are actually implemented by the phase field method. However, it is not known a priori, whether the integrands are bounded independent of the choice of $\xi$. As the integrands contain derivatives of $u$, it is not even sufficient to assume that $u$ is bounded. Indeed, it is quite natural to expect that by letting $\xi$ tend to zero and thus approaching the limit of a non-differentiable $\phi$, the gradients of the solution $u$ could blow up. Thus, if the crucial assumption of boundedness is not fulfilled, it can neither be concluded that integrals tend to zero because the integrated volume does, nor that for $\partial V_{n}^{1}$ the approximation $\phi \underline{\mathbf{D}} \nabla u=\varepsilon \underline{\mathbf{D}} \nabla u \approx 0$ holds (for sufficiently small $\varepsilon$ ). In fact, it becomes obvious that the 
line of thoughts above is not sufficient, if one notes that it would also work without the factor $\phi$ in the volume integrals of equation 2.23), which corresponds to the additional prefactor $1 / \phi$ in equation $(2.19)$. As will be shown below, this prefactor is vital for the method to yield the desired result.

In a more recent publication [164, LI et al. showed that phase-field methods can be used to model a number of different boundary conditions, no-flux being a special case which can indeed implemented by equation (2.21). However, the mechanisms described below by which the phase-field method achieves its goal also explain some important details of the numerical implementation not provided by the very abstract proof in reference [164].

\section{Mechanism of boundary condition enforcement}

Rewriting equation (2.21), the terms of the original PDE and the influence of the phase field can be separated:

$$
\begin{aligned}
\frac{\partial u}{\partial t} & =f(u)+\frac{1}{\phi} \nabla \cdot(\phi \underline{\mathbf{D}} \nabla u) \\
& =f(u)+\nabla \cdot(\underline{\mathbf{D}} \nabla u)+\frac{1}{\phi}(\nabla \phi) \cdot(\underline{\mathbf{D}} \nabla u) \\
& =f(u)+\nabla \cdot(\underline{\mathbf{D}} \nabla u)+(\nabla(\ln \phi)) \cdot(\underline{\mathbf{D}} \nabla u),
\end{aligned}
$$

where "." in the last two equations is the dot product between two vectors. In equation (2.27), the third term only becomes active, if there are components of the current density $-\underline{\mathbf{D}} \nabla u$ perpendicular to the boundary (i.e. violating the intended no-flux boundary condition), as $\nabla(\ln \phi)$, by definition of the phase field, is just a scaled normal vector:

$$
\nabla(\ln \phi)=\frac{\partial \ln \phi}{\partial n} \mathbf{n}
$$

With this information, equation 2.27 reads:

$$
\frac{\partial u}{\partial t}=f(u)+\nabla \cdot(\underline{\mathbf{D}} \nabla u)+\frac{\partial \ln \phi}{\partial n} \mathbf{n} \cdot(\underline{\mathbf{D}} \nabla u)
$$

Note that the second term resembles an advection term in fluid dynamics, though the velocity field is not divergence free. Shifting $\underline{\mathbf{D}}$ to the other side of the scalar product, the velocity field for the quantity $u$ is given by

$$
-\frac{\partial \ln \phi}{\partial n} \underline{\mathbf{D}} \mathbf{n}
$$

As $\underline{\mathbf{D}}$ is positive definite, $\mathbf{n} \cdot \underline{\mathbf{D}} \mathbf{n} \geq 0$. Taking $\mathbf{n}$ to point out of the domain $\mathcal{D}$, according to figure 2.2 c) $\partial \ln \phi / \partial n<0$ holds, which means that the velocity (2.30) points into the same half-space as $\mathbf{n}$, although it might not be normal to $\partial \mathcal{D}$. Therefore, $u$ is "advected" outwards with a velocity proportional to $|\mathrm{d} \ln \phi / \mathrm{d} n|$.

To show that the assumed boundedness of the integrands in equation 2.23 is actually present, the so-called maximum principle will be used: If the local reaction term in equation 2.21) is ignored for the moment, a pure diffusion equation is obtained. The maximum principle states that the solution $u(\mathbf{x}, t)$ for $0 \leq t<T$ and $\mathbf{x} \in \mathcal{D}_{c}$ is attained either on the 
spatial boundary $\partial \mathcal{D}_{c}$ or at some point $\mathrm{x} \in \mathcal{D}_{c}$ at $t=0$ [165]. Another way to say this is: The solution will never exceed the maximum of the initial field $u$ at $t=0$, provided that the values on the boundary $\partial \mathcal{D}_{c}$ stay below the maximum as well. The same applies to the minimum. For the purpose of the following reasoning, it is assumed that the boundary $\partial \mathcal{D}_{c}$ of the auxiliary domain is subject to a passive boundary condition which never attains a maximum or minimum, for example a Dirichlet condition $u=\langle u(t=0)\rangle_{\mathcal{D}_{c}}$ on $\partial \mathcal{D}_{c}$. Below, it will be argued why the boundary condition on the auxiliary domain is not important for the solution $u$ in the physical domain $\mathcal{D}$.

Having the maximum principle at hand, an upper bound for the left hand side of equation 2.23) can be obtained by differentiating equation (2.21) (still without the local reaction term) with respect to $t$ and noting that the time derivative $\frac{\partial u}{\partial t}$ itself therefore fulfills the diffusion equation

$$
\frac{\partial \frac{\partial u}{\partial t}}{\partial t}=\frac{1}{\phi} \nabla \cdot\left(\phi \underline{\mathbf{D}} \nabla \frac{\partial u}{\partial t}\right)
$$

The initial condition $\left.\frac{\partial u}{\partial t}\right|_{t=0}$ is connected to the initial condition $u_{0}(\mathbf{x})=u(\mathbf{x}, t=0)$ of the original diffusion equation via equation (2.21) or equation (2.29). Given that $u_{0}$ fulfills the no-flux boundary condition in the interface region, the $\phi$-dependent terms in equation 2.29 drop out and thus $\frac{\partial u}{\partial t}$ is bounded independent of $\phi$ by the maximum principle for all $t$ :

$$
\left|\frac{\partial u}{\partial t}\right|_{(\mathbf{x}, t)} \mid \leq C \quad \forall \mathbf{x}, t \quad \text { and } \quad \forall \xi .
$$

In the context of excitable media, with the source term $f=I_{\text {ion }}$ introducing additional $\frac{\partial u}{\partial t}$ contributions, usually, the fastest time scale of $u$ is given by the rise velocity upon activation. The maximum principle therefore ensures that $\partial u / \partial t$ is not increased further by the $\phi$-dependent diffusion dynamics and $C$ can be interpreted as the upstroke velocity of the action potential.

The boundedness of the integrand involving the reaction term $f(u)$ in equation 2.23 is seen immediately, because $u$ itself is subject to a diffusion equation and thus obeying the maximum principle, if $f(u)$ is omitted in equation (2.21). Again, this means that the $\phi$-dependent diffusion dynamics cannot increase or decrease $u$ further than determined by the reaction dynamics. Therefore $u$ is bounded and so is $f(u)$.

For the remaining terms on the right-hand side of equation (2.23), a simpler special case is considered, namely that of a diffusion tensor $\underline{\mathbf{D}}$ which is nearly spatially constant in the interface region and of an approximately straight boundary, such that spatial derivatives of $\underline{\mathbf{D}}$ and $\mathbf{n}$ can be neglected ${ }^{4}$ Under these assumptions, differentiating equation 2.29

\footnotetext{
${ }^{4} \underline{\mathbf{D}}$ constant has to be required only in the interface region, because it is reasonable to assume that the dynamical system, uninfluenced by the phase field, only produces finite spatial gradients in the physical domain $\mathcal{D}$. Anything else would render numerical simulations (even without the phase field method) impossible in any case. Therefore, here, it is sufficient to show that these gradients are not amplified by the choice of the steepness parameter $\xi$ of $\phi$. Furthermore, the assumption of a nearly straight boundary is probably not as stringent as it might first seem, as on the spatial scale of $\xi$, every boundary with finite curvature becomes straight for $\xi \rightarrow 0$. However, this generalization will not be shown here.
} 
in the normal direction yields:

$$
\begin{aligned}
\frac{\partial \frac{\partial u}{\partial n}}{\partial t} & =\nabla \cdot\left(\underline{\mathbf{D}} \nabla \frac{\partial u}{\partial n}\right)+\frac{\partial \ln \phi}{\partial n} \mathbf{n} \cdot\left(\underline{\mathbf{D}} \nabla \frac{\partial u}{\partial n}\right)+\frac{\partial^{2} \ln \phi}{\partial n^{2}} \mathbf{n} \cdot(\underline{\mathbf{D}} \nabla u) \\
& =\frac{1}{\phi} \nabla \cdot\left(\phi \underline{\mathbf{D}} \nabla \frac{\partial u}{\partial n}\right)+\frac{\partial^{2} \ln \phi}{\partial n^{2}} \mathbf{n} \cdot(\underline{\mathbf{D}} \nabla u)
\end{aligned}
$$

If only the first term on the right hand side was present, $\frac{\partial u}{\partial n}$ would be bounded by the maximum principle. Due to the shape of the phase field (compare figure 2.2 and the beginning of this section), $\frac{\partial^{2} \ln \phi}{\partial n^{2}}$ is negative everywhere in the interface region and approaching zero from below in the outward direction. Therefore, the second term on the right hand side is only present in the interface region and opposite in sign to the normal component of the flux $\underline{\mathbf{D}} \nabla u$. As $\underline{\mathbf{D}}$ is positive definite, the induced change of $\partial u / \partial n=\mathbf{n} \cdot \nabla u$ leads to a reduction of the normal component of $\underline{\mathbf{D}} \nabla u$, because in general, for a vector $\mathbf{v}$ with a normal component $v_{n}$

$$
\frac{\partial \mathbf{n} \cdot \underline{\mathbf{D}} \mathbf{v}}{\partial v_{n}}=\mathbf{n} \cdot \underline{\mathbf{D}} \mathbf{n} \geq 0
$$

which means that the change of the normal component of $\underline{\mathbf{D}} \mathbf{v}$ is positive, if the normal component of $\mathbf{v}$ is increased. The second term in equation 2.34 can therefore be viewed as a feedback term that aims at reducing $\mathbf{n} \cdot \underline{\mathbf{D}} \nabla u$ to zero by changing $\mathbf{n} \cdot \nabla u$ appropriately. This can in principle mean that $|\mathbf{n} \cdot \nabla u|$ is increased (if $\underline{\mathbf{D}}$ is anisotropic), but zero flux will always be reached at finite values, as indicated above, depending on the parallel components of $\nabla u$. As the phase field transition length $\xi$ tends to zero, the "feedback strength" is increased. Applying the same strategy for the derivative of $u$ parallel to the boundary yields equations for $\mathbf{p} \cdot \nabla u=\partial u / \partial p$ identical to equation (2.34), only without the last term. Therefore, this component is bounded by the maximum principle.

In summary, the gradient $\nabla u$ will thus be bounded independent of the steepness of the phase field at the boundary of $\mathcal{D}$ (at least in the approximations used here), which renders the line of thoughts after equation (2.23) correct. In addition to verifying the validity of the phase field approach, the above reasoning contains some useful information about the mechanisms, by which the no-flux boundary condition is achieved: Most importantly, one ambiguity of the ansatz 2.19) was removed. It is now clear why simply multiplying $\underline{\mathbf{D}}$ by the phase-field $\phi$ does not lead to the desired results, although equation (2.23) apparently suggested this. If this was done, equation 2.29 would contain $\partial \phi / \partial n$ instead of $\partial \ln \phi / \partial n$ (in addition to a factor $\phi$ before the diffusion term, which would, however, not have any impact). Replacing $\ln \phi$ by $\phi$ in equation 2.34 would destroy the gradient reduction effect of the second term. Therefore, it would not be possible to conclude that the gradient is bounded.

Furthermore, when the constant $C$ in equation 2.32 is interpreted as the upstroke velocity, as suggested, it can be seen from equation 2.23) that the accuracy of the method depends on this maximum upstroke velocity. This was also noted in reference [134, without, however, taking into account the possibility that this might be caused by the phase field method itself. This has now been ruled out by the line of thoughts above. An additional source of inaccuracy stems from the steepness of gradients arriving at the interface region from inside the domain $\mathcal{D}$. Equation 2.34 indicates that the boundary condition 
of the original problem formulated for $\mathcal{D}$ has been replaced by a dynamical process that aims at reducing $\mathbf{n} \cdot(\underline{\mathbf{D}} \nabla u)$ to zero. As this does not happen instantaneously, larger initial gradients mean larger remainders of $\mathbf{n} \cdot(\underline{\mathbf{D}} \nabla u)$ after the reduction process. In excitable media reaction-diffusion systems, the upstroke velocity and the steepness of gradients are, of course, closely related.

Finally, equation (2.29) together with the information illustrated in figure 2.2.) indicates that the velocity, with which the quantity $u$ is transported outwards, increases steadily in the interface region and becomes $\approx 1 / \xi$ after some distance. The smaller $\xi$, the less information can therefore travel back from $\mathcal{D}_{c} \backslash \mathcal{D}$ to $\mathcal{D}$. The only process available for this is the diffusion term in equation (2.29), which does not depend on $\phi$ and is therefore arbitrarily slow. This means that the auxiliary (arbitrary) boundary condition applied for numerical reasons at the boundary $\partial \mathcal{D}_{c}$ of the auxiliary domain becomes less and less important. This fact is useful for the numerical implementation of the phase field method (see section 2.2.3).

\section{Extension to other boundary conditions}

Although the phase-field method was designed by FENTON et al. to implement no-flux boundary conditions on arbitrary geometries, with the information above, it can be extended to a more general type of Neumann boundary conditions of the type

$$
\mathbf{n} \cdot \underline{\mathbf{D}} \nabla u=\mathbf{n} \cdot \mathbf{E},
$$

where $\mathbf{E}$ is a fixed vector. This is a special case of equation (2.16), when $\underline{\sigma}_{o} \nabla v_{o}$ is a constant electric field vector. It is seen immediately from the line of thoughts after equation 2.22 that the corresponding phase field replacement in this case is

$$
\nabla \cdot(\underline{\mathbf{D}} \nabla u) \quad \rightarrow \quad \frac{1}{\phi} \nabla \cdot(\phi(\underline{\mathbf{D}} \nabla u-\mathbf{E})) .
$$

With this substition, equation 2.21 is not altered for regions where $\phi \approx 1$, since $-\nabla \cdot \mathbf{E}=$ 0 . In the integrals of equation (2.23), however, the additional term appears, leading to $\mathbf{n} \cdot(\underline{\mathbf{D}} \nabla u-\mathbf{E})=0$ in equation $(2.25)$, which is the desired boundary condition. The same result can be obtained from reference [164] by starting from equations 2.28 and 2.37 and substituting the correct boundary condition.

\subsubsection{Models}

So far, in this chapter, the cross-membrane current $I_{\text {ion }}\left(V_{m}, \mathbf{h}\right)$ in, e.g., equations 2.4, 2.9 or 2.13 has been treated as an unknown quantity whose dynamics is determined by the membrane potential $V_{m}$ and an additional set of equations for the local (i.e. spatially uncoupled) variables $\mathbf{h}$. As described in section 2.1.1, one approach to construct this term is to empirically determine the contributions of all different ion species and membrane compounds that lead to transmembrane currents. Indeed, many models based on this bottom-up approach exist, although they are by definition based on incomplete data. A comprehensive list of physiological models, ordered by tissue type (atrial/ventricular) and species can be found in reference [166]. One advantage of this approach is that model pa- 
rameters can directly be related to physiological properties, which enables investigations of genetic defects and facilitates the adaption of the model for disease conditions. However, a major drawback of these models is their computational cost, due to the large number of local variables used. One extreme example is the Wang-Sobie model for neonatal rat ventricular myocytes [153] with 35 dynamic variables and 74 parameters. Although the large number of parameters might be beneficial for the reasons mentioned above, it is at the same time hindering generic approaches such as parameter scans or fitting procedures. Also, for most physiological models, sensitivity of the dynamics to individual parameters is not documented, which further complicates the analysis.

On the other end of the spectrum, generic models are created using a top-down approach to the model construction problem. Rather than incorporating every possible detail of the system for which information exists, they try to mimic the basic properties of cardiac tissue (or even more generic: of excitable media) using the fewest number of variables and parameters possible to achieve a particular degree of realism. A very generic model for excitable media is the Barkley model [72, which consists of only two variables and three parameters which determine the basic properties of the medium such as the excitability, excitation threshold and duration. The Barkley model will be used in this work for numerical simulations, when values of physiological parameters are not important or unknown and when results are to be obtained for the large class of excitable media. In all other simulations, the somewhat more detailed three-variable Fenton-Karma model [84] will be used, which has 14 parameters that enable it to reproduce important aspects of the dynamics of more realistic cardiac models.

\section{The Barkley and the Bär-Eiswirth model}

This two-variable, generic model was developed by BARKLEY in the beginning of the 1990s in an effort to understand spiral wave dynamics in generic excitable media. Although already used in reference [72, it was newly introduced in its most efficient numerical form in reference [167]. Its equations are

$$
\begin{aligned}
& \frac{\partial u}{\partial t}=\frac{1}{\epsilon} u(1-u)\left(u-\frac{v+b}{a}\right)+\nabla^{2} u \\
& \frac{\partial v}{\partial t}=g(u, v),
\end{aligned}
$$

with the parameters $\epsilon, a$ and $b$. For the original Barkley model, $g(u, v)=u-v$. The fast variable $u$ can be identified as a normalized $V_{m}$ in the mono-domain formalism of section 2.1.3, while the local part on the right hand side of the $u$-equation represents the cross-membrane current term $-I_{\text {ion }} / C_{m}$. There is only one local variable, such that $\mathbf{h}$ is actually a scalar and $h=v$. The diffusion tensor is normalized to $\underline{\mathbf{D}}=\mathrm{Id}$. An example of the local dynamics of the model, i.e. without the diffusion term, following a series of stimulations is shown in figure 2.4. It can be understood from the $(u, v)$ phase space structure, which is typical for simple excitable system: 5 and is depicted in figure 2.5 . It has a stable fixed point at $(0,0)$, whose basin of attraction is the whole square $[0,1[\times[0,1]$,

\footnotetext{
${ }^{5}$ Another example for a two-variable system with a similar phase space structure is the FitzHughNagumo model [168, 169, which is a reduced version of the nerve axon model constructed by Hodgkin and Huxley.
} 


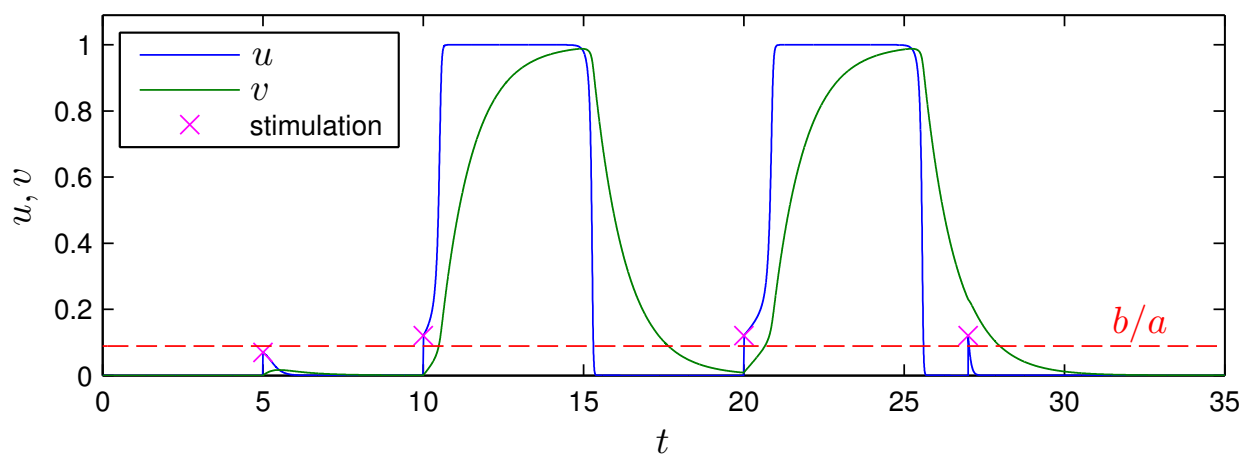

Figure 2.4: Barkley model dynamics. Four attempts, marked by " $\times$ ", to induce an excitation in the Barkley model (without diffusion) by instantaneously setting the model variable $u$. In the first case, the stimulation is subthreshold. The second and third stimulation produce almost identical excitations, whereas the fourth stimulation occurs at a time when excitability is not fully recovered yet, as $v$ has not returned to zero. Because of this refractoriness, the stimulation fails to produce an excitation. Model parameters: B1 (see table A.1, page 177).

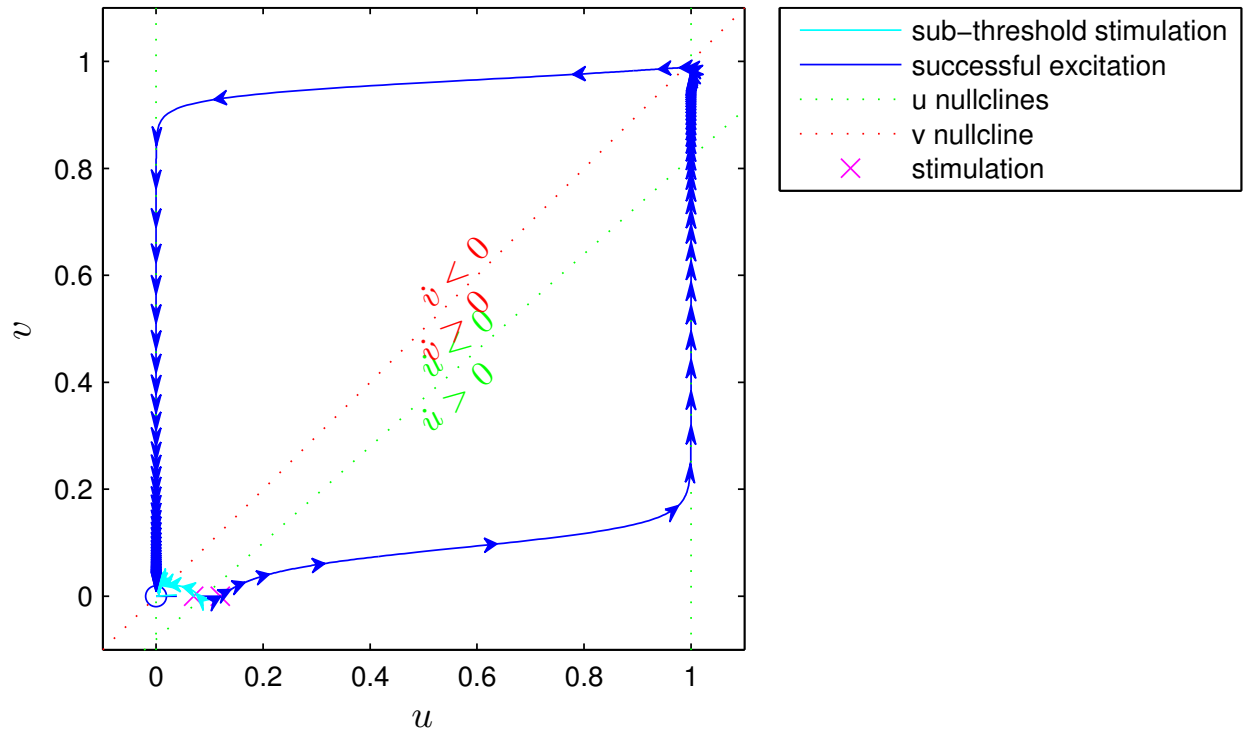

Figure 2.5: Barkley model: local phase space. Shown are two stimulation attempts from the fully recovered state: one is subthreshold (cyan), causing the system to return immediately to the stable fixed point at $(0,0)$, whereas the other one is far enough in the $\dot{u}>0$ region to produce an excitation (blue). Arrows on the trajectories mark equidistant time points, indicating the fast dynamics of $u$ compared to $v$. The tilted $u$ nullcline has the equation $v=a u-b$ and is responsible for the refractoriness shortly after an excitation, when still $v>0$, but $u \approx 0$ : in this case, the distance to the right necessary to cross the $u$ nullcline is considerably increased. When $v>a-b$, the system is in absolute refractoriness, because then for every $u \in[0,1[$ it is $\dot{u}<0$. Model parameters: B1 (see table A.1, page 177). 
but trajectories starting a considerable distance ${ }^{6}$ right of the nullcline $u=(v+b) / a$ make a large detour close to $u=1, v=1$ and $v=0$ before returning to the fixed point. In contrast, trajectories starting close to $(u, v)=(0,0)$ return directly. The first case corresponds to action potential following a superthreshold depolarization, whereas the second is a subthreshold depolarization not strong enough to evoke an action potential. The meaning of the three parameters in terms of the characteristics of excitable media can readily be read off the phase space diagram: $b / a$ is the excitation threshold during rest, whereas $\epsilon$ determines the time scale of $u$ compared to $v$. Its main impact is on the upstroke velocity of an action potential, which also determines the conduction velocity in the spatially extended case in conjunction with diffusion. $a$ on its own mainly influences the action potential duration, as it determines the slope of the above-mentioned nullcline and thus the value of $v$ at which $u$ starts to decrease.

Despite its simplicity, the Barkley model (now, again, including the Laplacian term) supports a large number of different wave patterns, including pulses, plane waves and both stationary and meandering spiral waves. However, there are no (homogeneous) parameters for which the Barkley displays spiral wave breakup or other types of chaos. It was shown by B̈̈R and EISWIRTH that turbulence can be observed by delaying the increase of the slow variable $v$. This can be done by redefining $g$ in equation (2.38) as follows [73]:

$$
g(u, v)= \begin{cases}-v & u<\frac{1}{3} \\ 1-6.75 u(u-1)^{2}-v & \frac{1}{3} \leq u \leq 1 \\ 1-v & u \geq 1\end{cases}
$$

This modification will be referred to as the Bär-Eiswirth model in the rest of this thesis.

\section{The Fenton-Karma model}

The equations for this generic model were developed by FENTON and KARMA in 1998 with the aim of capturing the most important features specific to cardiac models (as opposed to general excitable media). Consequently, although the model has only three variables, it considerably more tightly connected to the physiological mechanisms of excitability in the cardiac muscle. The model considers three cross-membrane currents, named fast inward (fi), slow outward (so) and slow inward (si) current. These roughly correspond to the sodium, potassium and calcium currents, respectively (cf. section 1.2.1). However, the complexity of different ion channel types and other contributions to membrane potential dynamics has been condensed into these net currents and their activation and inactivation. Therefore, the parameters (most of which are timescales and thresholds) have a physiological meaning in that they reflect typical or net properties of these summarized currents, but nevertheless they cannot be interpreted as descriptions of the microscopic components responsible for membrane potential dynamics.

\footnotetext{
${ }^{6}$ This distance is indeed finite and depends on the parameter $\epsilon$, which determines, for a fixed starting point of the trajectory, whether the $u$ dynamics will be successful in driving the system even more to the right and thus to performing an action potential, or whether the $v$ dynamics is dominant and brings the system back over the $u$-nullcline and thus directly to the fixed point.
} 
The equations of the Fenton-Karma model are

$$
\begin{aligned}
\frac{\partial u}{\partial t} & =\nabla \cdot \underline{\mathbf{D}} \nabla u-J_{\mathrm{fi}}(u, v)-J_{\mathrm{so}}(u)-J_{\mathrm{si}}(u, w) \\
\frac{\partial v}{\partial t} & =\Phi\left(u_{c}-u\right)(1-v)\left(\frac{\Phi\left(u-u_{v}\right)}{\tau_{v 1}^{-}}+\frac{\Phi\left(u_{v}-u\right)}{\tau_{v 2}^{-}}\right)-\Phi\left(u-u_{c}\right) \frac{v}{\tau_{v}^{+}} \\
\frac{\partial w}{\partial t} & =\Phi\left(u_{c}-u\right) \frac{1-w}{\tau_{w}^{-}}-\Phi\left(u-u_{c}\right) \frac{w}{\tau_{w}^{+}}
\end{aligned}
$$

where the three cross-membrane currents are defined as

$$
\begin{aligned}
J_{\mathrm{fi}}(u, v) & =-\frac{v}{\tau_{d}} \Phi\left(u-u_{c}\right)(1-u)\left(u-u_{c}\right) \\
J_{\mathrm{so}}(u) & =\frac{u}{\tau_{0}} \Phi\left(u_{c}-u\right)+\frac{1}{\tau_{r}} \Phi\left(u-u_{c}\right) \\
J_{\mathrm{si}}(u, w) & =-\frac{w}{2 \tau_{s i}}\left(1+\tanh \left[k\left(u-u_{c}^{s i}\right)\right]\right) .
\end{aligned}
$$

The sum of these currents corresponds to the term $-I_{\text {ion }} / C_{m}$ of section 2.1.3. Again, $u$ is a normalized membrane potential $V_{m}$ and on the order 0 to 1 , such that one unit of $u$ roughly corresponds to a $100 \mathrm{mV}$ membrane potential change. The vector of local variables for the Fenton-Karma model therefore is $\mathbf{h}=(v, w)$.

In its original published form, the function $\Phi(x)$ in equations (2.40) and 2.41) is simply the step function

$$
\Phi(x)=\Theta(x):= \begin{cases}1 & x \geq 0 \\ 0 & x<0 .\end{cases}
$$

However, in this case, the right hand side of the dynamical system $(2.40)$ is not differentiable. When this is required, an continuous approximation for the step function will be used instead:

$$
\Phi(x)=\Theta^{*}(x):=\frac{1}{2}\left(1+\tanh \left(k_{2} x\right)\right)
$$

In this way, $k_{2}$ becomes an additional parameter of the model and the original dynamics is recovered for $k_{2} \rightarrow \infty$, which is why large values for $k_{2}$ will be used throughout this thesis. With this choice of $\Phi$, the equations will be referred to as the smooth FentonKarma model.

The steady state of the Fenton-Karma model is close to $(u, v, w) \approx(0,1,1)$, where $u$, even for the case $\Phi=\Theta$, deviates slightly from 0 because of the tanh term in equation (2.41c). If depolarized above the threshold $u_{c}$, the fast inward current $J_{\text {fi }}$ drives $u$ rapidly towards 1 , on a time scale defined by $\tau_{d}$. $J_{\text {so }}$ is responsible for repolarization, whereas $J_{\text {si }}$ is the current that counteracts $J_{\text {so }}$ during the plateau phase [84]. The local variables $v$ and $w$ are gates for the fast and slow inward currents, respectively. For the resting state, these two currents have their full amplitude, but time periods in which $u>u_{v}$ or $u>u_{c}$ bring $v$ and $w$ closer to 0 , thereby reducing the ability of the model membrane to depolarize or withstand the repolarizing slow outward current. This leads to both a decrease in membrane excitability (upstroke velocity) and action potential duration (APD), the former 


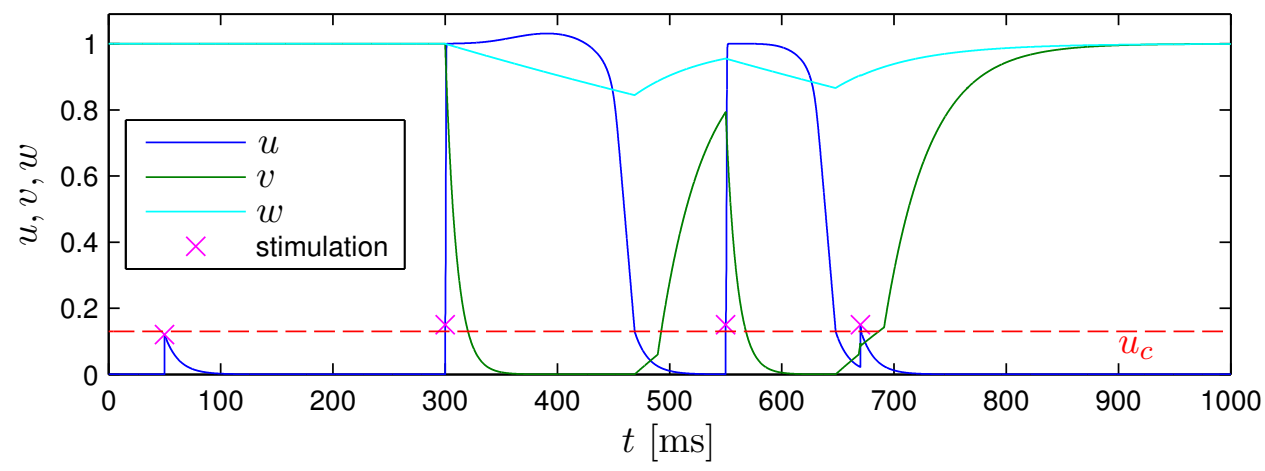

Figure 2.6: Fenton-Karma model dynamics. Four attempts, marked by " $\times$ ", to induce action potentials in the Fenton-Karma model (without diffusion) by instantaneously setting the model variable $u$. In the first case, the stimulation is subthreshold. The second and third stimulation each produce an excitation, but the APD varies due to restitution. The fourth stimulation occurs during refractoriness and fails to produce an action potential. Model parameters: FK1 (see table A.2, page 177).

correlating with conduction velocity $(\mathrm{CV})$.

As both kinds of refractoriness are parameterized separately with corresponding thresholds and time scales, the Fenton-Karma model is able to reproduce a variety of CV and APD restitution curves, even for more complex models as well as experimentally measured restitution curves. Indeed, providing a simple (as opposed to ionically accurate) model with realistic restitution properties was one of the primary reasons for its construction [84. Later, an extended version with four variables was published which, in addition to the restitution properties, also produces more accurate action potential shapes [163]. In summary, despite its lack of detail on the ionic level, the Fenton-Karma model represents an intermediate class of models that is still generic but accounts for some important properties specific to cardiac tissue. An example trajectory of the local model dynamics is shown in figure 2.6. It should be noted that the meaning of the parameters $\tau_{v 1}^{-}$and $\tau_{v 2}^{-}$ is reversed in reference [104] with respect to the original model publication in reference [84]. In this work, the original definition is used.

\subsubsection{Spiral tips and phase singularities}

In contrast to plane waves which travel through a given finite domain only once and selfterminate when they arrive at a boundary, spiral waves have, in principle, an unlimited life span. Both the Barkley model and the Fenton-Karma model can exhibit this fundamental excitation pattern of excitable media. The inherent stability of spiral waves is one of the key reasons for their dangerousness in the context of cardiac dynamics (see section 1.4). Even during the chaotic activity of fibrillation, activity has been shown to be organized around phase singularities [42, 43].

Spiral waves can, in general, extend over an arbitrarily large domain. However, unless the spiral is perturbed very close to its center (the spiral tip), it will continue to rotate largely uninfluenced. The organizing centers around which spiral waves rotate can be characterized by phase singularities. A suitable phase for the detection of phase singularities can be defined in different ways. In the two-variable Barkley model, this is particularly simple, 
a)

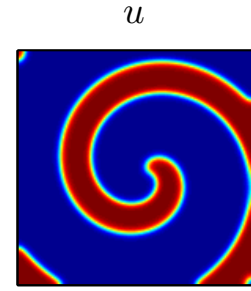

b)

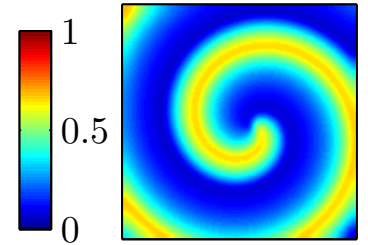

c)

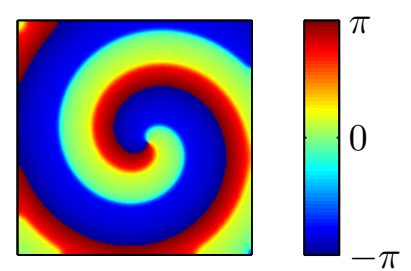

Figure 2.7: Spatial phase distribution. a), b) Exemplary distributions of the $u$ and $v$ variables of the Barkley model during spiral wave dynamics. c) Distribution of the phase $\theta$. Near the center of the domain, all phases converge in a single point - the phase singularity. Simulation of the Barkley model using parameter set B1 (see table A.1, page 177 .

since the trajectory of a normal activation in the local phase space is similar enough to a circle to define a phase $\theta$ by the function [170]

$$
\theta(\mathbf{x}, t)=\arctan 2\left(u(\mathbf{x}, t)-u^{*}, v(\mathbf{x}, t)-v^{*}\right)
$$

where $\left(u^{*}, v^{*}\right)$ is a point in the local phase space which is circled by the trajectory during a normal activation. For the example shown in figure 2.5. $\left(u^{*}, v^{*}\right)=(0.3,0.3)$ would be an appropriate choice, although one has to ensure that the values are still adequate when looking at the dynamics of a single point embedded into the extended system. An example for this definition of the phase and the resulting phase distribution in space are shown in figure 2.7 for a spiral wave in the Barkley model. Close to the center of the domain, all different phases $\theta \in]-\pi, \pi]$ are present. This point defines the phase singularity. When the model has more than two variables, one can select two suitable variables or two different combinations of variables to define the phase according to equation (2.44). If this is not straightforward or, e.g., only the membrane potential is available experimentally, delay embeddings (or, almost equivalently, the derivative) of the membrane potential or Hilbert transformations can be used to obtain a second variable for the definition of a phase [170, 171, 172]. In this thesis, spiral tip detection will only be carried out for the Barkley model, so the above method is sufficient.

In a spatial domain $\mathcal{D}$ with two dimensions and with a suitable definition of the phase, the topological charge $n^{\text {top }}$ can be defined as 173 .

$$
n^{\text {top }}=\frac{1}{2 \pi} \oint_{\partial \mathcal{D}} \nabla \theta \mathrm{d} \mathbf{l},
$$

where $\mathbf{l}$ is the path parameterizing the boundary $\partial \mathcal{D}$ of $\mathcal{D} . \nabla \theta$ is well-defined (except at the phase singularity itself) if the phase interval $]-\pi, \pi]$ is interpreted as having periodic 
boundary conditions, or equivalently, if for each point in space, the branches of the arctan2 function are chosen appropriately. Assuming that the phase $\theta$ is differentiable everywhere in $\mathcal{D}$, equation 2.45 trivially yields $n^{\text {top }}=0$ due to Stokes' theorem. However, integrating once around a phase singularity leads to a line integral of $\pm 2 \pi$, which is possible despite Stokes' theorem, because $\theta$ cannot be made differentiable everywhere. The topological charge of a domain with one phase singularity is thus $n^{\text {top }}= \pm 1$, where the sign indicates the chirality of the phase singularity, which, for the same definition of the phase, characterizes the direction of rotation of the spiral wave. If multiple phase singularities are present in the domain within the integration path, a net topological charge is obtained. For the sake of detecting spiral tips, therefore, small integration domains have to be chosen (see numerical implementation of spiral tip detection in section 2.2.5).

It should be noted that there are other ways of finding spiral wave tips which do not rely on the definition of a phase. One possibility is to find the intersection of the iso-lines for two different variables. These two variables can either be two model variables or a second variable can be obtained using one of the methods mentioned above. A common choice for the detection of spiral tips and their three-dimensional equivalents, filaments of scroll waves, is $\dot{V}_{m}$ as the second iso-line or iso-surface (see appendix B of reference [84]). However, these methods of intersection do not reveal any information about the chirality of the spiral waves.

\subsubsection{Lyapunov stability analysis}

Let

$$
\frac{\mathrm{d} \mathbf{u}}{\mathrm{d} t}=\mathbf{f}(\mathbf{u}) \quad \text { with } \quad \mathbf{u}\left(t_{0}\right)=\mathbf{u}_{0}
$$

be a bounded, continuous dynamical system 7 with differentiable $\mathbf{f}: \mathbb{R}^{n} \rightarrow \mathbb{R}^{n}$ and $\mathbf{u} \in \mathbb{R}^{n}$, where $n$ is the phase space dimension. Lyapunov stability analysis can make a statement about overall stability properties of an attractor and quantifies the "sensitive dependence on initial conditions" that is often viewed as a defining criterion for chaos. This is done by analyzing the behavior of small perturbations with respect to a reference trajectory under the assumption that the system is ergodic. An attractor is regarded as stable, when perturbations in arbitrary directions in phase space do not, in the long run, grow with time, i.e. all perturbed trajectories decay back to the reference trajectory or at least do not diverge from it. The analysis is carried out in the tangent space of the dynamics, i.e. up to linear order. As will be described below in detail, this approximation in general leads to different exponential growth rates in distinct directions in tangent space, i.e. a perturbation $\delta \mathbf{u}$ initialized at time $t_{0}$ grows approximately like $\delta \mathbf{u}\left(t_{0}+t\right)=\delta \mathbf{u}\left(t_{0}\right) \exp (\lambda t)$ for large $t$. The growth rate $\lambda$, which depends on direction of the initial perturbation, is the so-called Lyapunov exponent associated with this direction. The scenario for a specific direction in phase space is illustrated in figure 2.8. Note, however, that in reality, the behavior of the perturbations can vary substantially across different regions of the attractor. Lyapunov exponents are therefore long-term, average growth rates.

\footnotetext{
${ }^{7}$ In allusion to some of the excitable media models used in this thesis, the letter $\mathbf{u}$ refers here to a state in phase space (instead of the more widely-used $\mathbf{x}$ ) to avoid confusion with a position $\mathbf{x} \in \mathbb{R}^{d}$ in space (with $d=1,2,3$ ).
} 


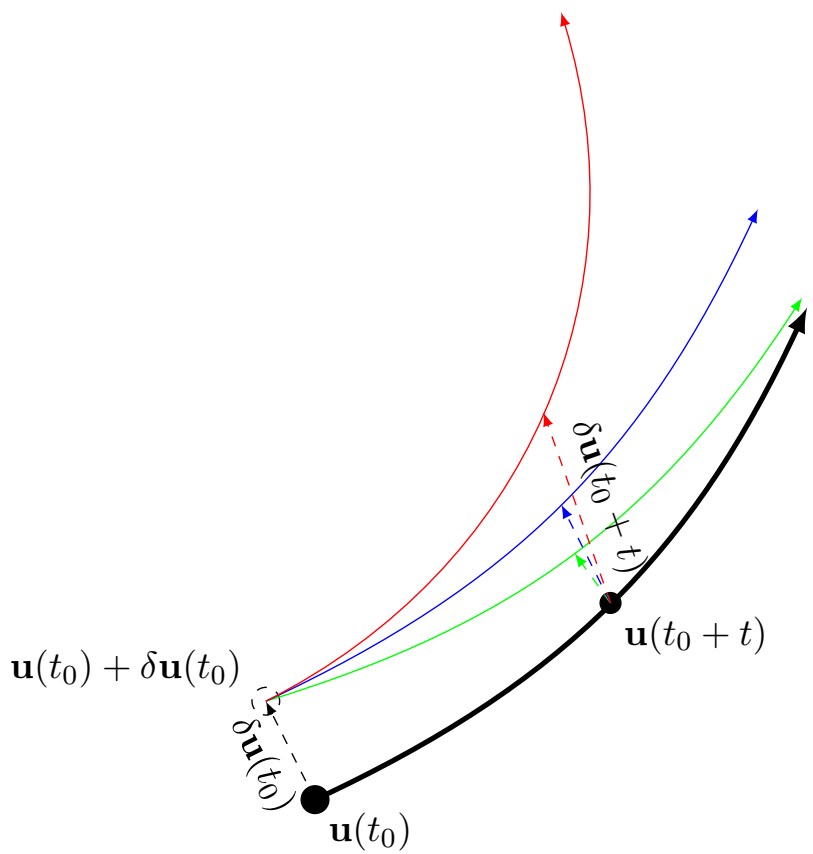

Figure 2.8: Lyapunov stability analysis extracts growth rates of perturbations. The black line represents a reference trajectory $\mathbf{u}\left(t_{0}+t\right)$, which is a solution of the dynamical system. An infinitesimally perturbed initial condition (dashed circle) leads to different trajectories. On average, for $t \rightarrow \infty$, the perturbation $\delta \mathbf{u}$ with respect to the reference trajectory either shrinks exponentially (green line; $\lambda<0$ ), stays constant (blue line; $\lambda=0$ ) or grows exponentially (red line; $\lambda>0$ ).

In the following, a very condensed summary of the mathematical definition of Lyapunov exponents and vectors will be given, facilitating the numerical implementation of the analysis (see section 2.2.6) and the interpretation of the results in section 3.1. Instead of working through this rather technical and mathematical section, some readers might prefer to skip directly to the next section (page 57) and obtain a more intuitive understanding of the meaning of Lyapunov exponents and vectors while reading the results in section 3.1.

\section{Definition of Lyapunov exponents}

Let

$$
\begin{array}{rlcc}
\Phi^{t}:\left(t, \mathbf{u}_{0}\right) & \mapsto & \mathbf{u}\left(t_{0}+t\right) \\
\mathbb{R} \times \mathbb{R}^{n} & \rightarrow & \mathbb{R}^{n}
\end{array}
$$

be the flow of the system, where the first argument $t$ is usually written as a superscript and which is alternatively understood as a family of functions $\Phi^{t}: \mathbb{R}^{n} \rightarrow \mathbb{R}^{n}$ parametrized by $t$. Combining equations (2.46) and (2.47), this definition is equivalent to the statement

$$
\left.\frac{\partial \Phi^{t}}{\partial t}\right|_{t^{\prime}}=\mathbf{f} \circ \Phi^{t^{\prime}} \quad \text { with } \quad \Phi^{0}=\operatorname{Id}_{\mathbb{R}^{n}} .
$$

Given a reference trajectory $\mathbf{u}\left(t_{0}+t\right)$, we can ask how an infinitesimally perturbed trajectory with an initial condition $\mathbf{u}_{0}+\delta \mathbf{u}_{0}$ instead of $\mathbf{u}_{0}$ evolves. Up to linear order the 
following identity holds

$$
\frac{\partial \delta \mathbf{u}}{\partial t}=\frac{\partial(\mathbf{u}+\delta \mathbf{u})}{\partial t}-\frac{\partial \mathbf{u}}{\partial t}=\mathbf{f}(\mathbf{u}+\delta \mathbf{u})-\mathbf{f}(\mathbf{u}) \approx \mathbf{f}(\mathbf{u})+\mathrm{J}_{\mathbf{f}}^{\mathbf{u}} \delta \mathbf{u}-\mathbf{f}(\mathbf{u})=\mathrm{J}_{\mathbf{f}}^{\mathbf{u}} \delta \mathbf{u},
$$

where $\mathrm{J}_{\mathbf{f}}^{\mathbf{u}}$ denotes the Jacobian of $\mathbf{f}$ at the point $\mathbf{u} \in \mathbb{R}^{n}$. Alternatively, we can use the flow with the perturbed initial condition to obtain an approximation for the temporal evolution of the perturbation $\delta \mathbf{u}$ :

$$
\delta \mathbf{u}^{*}\left(t_{0}+t\right)=\mathbf{u}\left(t_{0}+t\right)+\delta \mathbf{u}^{*}\left(t_{0}+t\right)-\mathbf{u}\left(t_{0}+t\right)=\Phi^{t}\left(\mathbf{u}_{0}+\delta \mathbf{u}_{0}\right)-\Phi^{t}\left(\mathbf{u}_{0}\right) \approx \mathrm{J}_{\Phi^{t}}^{\mathbf{u}_{0}} \delta \mathbf{u}_{0}
$$

To see that equation 2.50) actually describes the same dynamics as equation 2.49), we expand the time derivative of $\mathrm{J}_{\Phi^{t}}^{\mathbf{u}_{0}}$. Assuming all the partial derivatives of the flow are continuous and thus commute, we have

$$
\left.\frac{\partial \mathrm{J}_{\Phi^{t}}^{\mathbf{u}_{0}}}{\partial t}\right|_{t^{\prime}}=\mathrm{J}_{\left.\frac{\partial \Phi}{\partial t}\right|_{t^{\prime}}}^{\mathbf{u}_{0}} \stackrel{2.48)}{=} \mathrm{J}_{\mathbf{f} \circ \Phi^{t^{\prime}}}^{\mathbf{u}_{0}}=\mathrm{J}_{\mathbf{f}}^{\Phi^{t^{\prime}}\left(\mathbf{u}_{0}\right)} \circ \mathrm{J}_{\Phi^{t^{\prime}}}^{\mathbf{u}_{0}}=\mathrm{J}_{\mathbf{f}}^{\mathbf{u}\left(t_{0}+t^{\prime}\right)} \circ \mathrm{J}_{\Phi^{t^{\prime}}}^{\mathbf{u}_{0}}
$$

The composition operator $\circ$ for linear maps was deliberately used in the last two steps instead of ordinary matrix multiplication to emphasize the similarity of equation 2.51) to equation (2.48). A straightforward calculation shows that $\mathrm{J}_{\Phi^{0}}^{\mathbf{u}_{0}}=\operatorname{Id}_{\mathbb{R}^{n}}$. By comparing equation (2.48) with 2.46) and in the same way equation 2.51) with (2.49), we see that $\mathrm{J}_{\Phi^{t}}^{\mathbf{u}_{0}}$ is indeed the flow for the perturbations defined by equation $(2.49)$, i.e.

$$
\delta \mathbf{u}^{*}(t)=\delta \mathbf{u}(t)
$$

OSELEDEC's theorem [111] ensures under fairly general conditions that the limit

$$
\lim _{t \rightarrow \infty}\left(\mathrm{J}_{\Phi^{t}}^{\mathbf{u}_{0} T} \mathrm{~J}_{\Phi^{t}}^{\mathbf{u}_{0}}\right)^{1 / 2 t}=\boldsymbol{\Lambda}_{\mathbf{u}_{0}}
$$

exists for almost all initial conditions $\mathbf{u}_{0}$ on the attractor. The logarithms of the eigenvalues of $\boldsymbol{\Lambda}$ are called Lyapunov exponents and are denoted by $\lambda_{i}, i=1 \ldots n$, with the convention that $\lambda_{i} \geq \lambda_{j}$ for $i<j$. If we denote the unique Lyapunov exponents (i.e. without multiplicity) by $\lambda^{(i)}$, each of them is associated with the eigenspace $E^{(i)}$ belonging to the eigenvalue $\exp \left(\lambda^{(i)}\right)$, where $\operatorname{dim} E^{(i)}$ is the multiplicity of the Lyapunov exponent. In this alternative definition, $i$ ranges between 1 and $\tilde{n}$, where $\tilde{n}$ is the number of distinct Lyapunov exponents. One of the fundamental properties of Lyapunov exponents which makes them useful for the characterization of attractors is that, for ergodic systems, the eigenvalues of $\boldsymbol{\Lambda}_{\mathbf{u}_{0}}$ and thus the Lyapunov exponents are identical for almost al 8 initial conditions $\mathbf{u}_{0}$ and only the matrix itself and its eigenvectors really depend on $\mathbf{u}_{0}$. OsELEDEC also showed that these exponents can be obtained by looking at the linearized dynamics of single perturbations $\delta \mathbf{u}$, which are initialized in the right subset of tangent space:

$$
\lambda^{(i)}=\lim _{t \rightarrow \infty} \frac{1}{t} \ln \left\|\mathrm{J}_{\Phi^{t}}^{\mathbf{u}_{0}} \delta \mathbf{u}_{0}\right\| \quad \delta \mathbf{u}_{0} \in \mathfrak{E}^{(i)} \backslash \mathfrak{E}^{(i+1)} \subset \mathbb{R}^{n}
$$

\footnotetext{
${ }^{8}$ Exceptions are, e.g., unstable fixed points or unstable periodic orbits embedded into an attractor.
} 
$\mathfrak{E}^{(i)}$ is the subspace of $\mathbb{R}^{n}$ which is spanned by eigenvectors for which $\lambda \leq \lambda^{(i)}$ :

$$
\mathfrak{E}^{(i)}=\operatorname{span}\left(E^{(i)}, E^{(i+1)}, \ldots, E^{(\tilde{n})}\right)
$$

and for consistency we define $\mathfrak{E}^{(\tilde{n}+1)}:=\{0\}$. Let $U^{p} \in \mathbb{R}^{n}$ be an arbitrary set which is contained and open in the $p$-dimensional subspace spanned by linearly independent vectors $\delta \mathbf{u}_{0}^{r_{k}} \in \mathfrak{E}^{\left(r_{k}\right)} \backslash \mathfrak{E}^{\left(r_{k}+1\right)}$ for $1 \leq k \leq p$ and $r_{k}$ suitable integer numbers. As an extension of equation (2.54), it can be shown that $U^{p}$ grows like the sum of the corresponding Lyapunov exponents [174, 175, 176]:

$$
\lim _{t \rightarrow \infty} \frac{1}{t} \ln \operatorname{Vol}\left(\mathrm{J}_{\Phi^{t}}^{\mathbf{u}_{0}} U^{p}\right)=\sum_{k=1}^{p} \lambda^{\left(r_{k}\right)}
$$

Since the actual choice of $U^{p}$ within the subspace does not matter, for simplicity, one can think of the parallelotope spanned by the $\delta \mathbf{u}_{0}^{r_{k}}$. Choosing $\operatorname{Vol}\left(U^{p}\right)=1$ is a convenient choice to assign $\operatorname{Vol}\left(\mathrm{J}_{\Phi^{t}}^{\mathbf{u}_{0}} U^{p}\right)$ the meaning of a growth factor even for finite $t$. Note however that, in the formulation of equations 2.54) and 2.55), the choice of $\delta \mathbf{u}_{0}$ or the $\delta \mathbf{u}_{0}^{r_{k}}$ requires a-priori knowledge of the subspaces $E^{(i)}$. Guessing these subspaces for all Lyapunov exponents but the first $(i=1)$ is nearly impossible because $\mathfrak{E}^{(i)}$ has zero-measure in $\mathbb{R}^{n}$ for $i>1$. Besides this systematic problem, numerical calculation is impeded by the fact that slight numerical errors will always lead to components of $\delta \mathbf{u}$ in $\mathfrak{E}^{(1)} \backslash \mathfrak{E}^{(2)}$, which composes almost all of $\mathbb{R}^{n}$. Therefore, any (even most carefully chosen) $\delta \mathbf{u}_{0}$ will asymptotically grow like $\exp \left(\lambda_{1} t\right)$.

In 1980, more than a decade after the rigorous mathematical definition of Lyapunov exponents by OSELEDEC (1968), BENETTIN and co-workers found a method for calculating all Lyapunov exponents [112, 113]. It makes use of two basic facts: Firstly, assuming $t=t(m)=m \Delta t$ with some $m \in \mathbb{N}$, the Jacobian $\mathrm{J}_{\Phi^{t}}^{\mathbf{u}_{0}}$ can be replaced by

$$
\mathrm{J}_{\Phi^{t}}^{\mathbf{u}_{0}}=\mathrm{J}_{\Phi^{\Delta t}}^{\mathbf{u}\left(t_{0}+(m-1) \Delta t\right)} \mathrm{J}_{\Phi^{\Delta t}}^{\mathbf{u}\left(t_{0}+(m-2) \Delta t\right)} \cdots \mathrm{J}_{\Phi^{\Delta t}}^{\mathbf{u}_{0}} .
$$

Secondly, like almost any $\delta \mathbf{u}_{0}$ will be in $\mathfrak{E}^{(1)} \backslash \mathfrak{E}^{(2)}$, similarly, almost any $p$-dimensional parallelotope $U^{p}$ defined by linearly independent $\delta \mathbf{u}_{0}^{k}, 1 \leq k \leq p$ will be in $\mathfrak{E}^{(1)} \backslash \mathfrak{E}^{\left(p^{*}+1\right)}$, where $p^{*}$ is chosen such that $\lambda^{\left(p^{*}\right)}=\lambda_{p}$. This means that, almost certainly, the $r_{k}$ in equation 2.55 refer to the $p$ most expanding directions:

$$
\lim _{t \rightarrow \infty} \frac{1}{t} \ln \operatorname{Vol}\left(\mathrm{J}_{\Phi^{t}}^{\mathbf{u}_{0}} U^{p}\right)=\sum_{k=1}^{p} \lambda_{k}
$$

Since we are dealing with linear maps and so the volume of all open sets in a subspace grows by the same factor, by virtue of equation (2.56), growth of the subspace $\operatorname{span}\left(\delta \mathbf{u}_{0}^{1}, \delta \mathbf{u}_{0}^{2}, \ldots, \delta \mathbf{u}_{0}^{p}\right)$ can be calculated as

$$
\begin{aligned}
\operatorname{Vol}\left(\mathrm{J}_{\Phi^{t}}^{\mathbf{u}_{0}} U^{p}\right) & =\operatorname{Vol}\left(\mathrm{J}_{\Phi^{\Delta t}}^{\mathbf{u}\left(t_{0}+(m-1) \Delta t\right.} U_{m-1}^{p}\right) \cdot \operatorname{Vol}\left(\mathrm{J}_{\Phi^{\Delta t}}^{\mathbf{u}\left(t_{0}+(m-2) \Delta t\right.} U_{m-2}^{p}\right) \cdots \operatorname{Vol}\left(\mathrm{J}_{\Phi^{\Delta t}}^{\mathbf{u}_{0}} U^{p}\right) \\
& =\prod_{l=0}^{m-1} \operatorname{Vol}\left(\mathrm{J}_{\Phi^{\Delta t}}^{\mathbf{u}\left(t_{0}+l \Delta t\right)} U_{l}^{p}\right) \quad \text { with } \quad U_{0}^{p}:=U^{p},
\end{aligned}
$$


where the parallelotopes fulfill $\operatorname{Vol}\left(U_{i}^{p}\right)=1$ for $1 \leq i \leq m-1$ and the $\delta \mathbf{u}_{i}^{k}, 1 \leq k \leq p$ spanning the parallelotope $U_{i}^{p}$ satisfy

$$
\operatorname{span}\left(\delta \mathbf{u}_{i+1}^{1}, \delta \mathbf{u}_{i+1}^{2}, \ldots, \delta \mathbf{u}_{i+1}^{p}\right) \equiv \operatorname{span}\left(J_{\Phi^{\Delta t}}^{\mathbf{u}\left(t_{0}+i \Delta t\right)} \delta \mathbf{u}_{i}^{1}, \mathrm{~J}_{\Phi^{\Delta t}}^{\mathbf{u}\left(t_{0}+i \Delta t\right)} \delta \mathbf{u}_{i}^{2}, \ldots, \mathrm{J}_{\Phi}^{\mathbf{u}\left(t_{0}+i \Delta t\right)} \delta \mathbf{u}_{i}^{p}\right) .
$$

The latter means that a suitable parallelotope can be chosen for each step of the linearized flow independently, as long as the new parallelotope is in the same subspace as the result from the last step $\left(\operatorname{Vol}\left(U_{i}^{p}\right)=1\right.$ was only chosen to avoid having to divide by the original volume in each step to obtain the growth factor).

As noted by Benettin, a convenient way to calculate the parallelotopes' volume and get a computationally favorable representation of the subspaces is by means of a reduced QR-decomposition: Let $\mathbf{A}^{i+1} \in \mathbb{R}^{n \times p}$ be a matrix whose columns are the $p$ linearly independent vectors spanning the parallelotope $J_{\Phi \Delta t}^{\mathbf{u}\left(t_{0}+i \Delta t\right)} U_{i}^{p} . \quad \mathbf{A}^{i+1}=\mathbf{Q}^{i+1} \mathbf{R}^{i+1}$ is a unique decomposition of $\mathbf{A}^{i+1}$ into a matrix $\mathbf{Q}^{i+1} \in \mathbb{R}^{n \times p}$ whose columns are orthonormal and an upper triangular matrix $\mathbf{R}^{i+1} \in \mathbb{R}^{p \times p}$ whose diagonal elements we require to be positive. Each column of $\mathbf{A}^{i+1}$ is thus expressed as a linear combination of the columns of $\mathbf{Q}^{i+1}$ whose weights are given by the corresponding column of $\mathbf{R}^{i+1}$. As $\mathbf{R}^{i+1}$ is upper triangular, the first $q$ columns of $\mathbf{Q}^{i+1}(1 \leq q \leq p)$ span the same subspace as the first $q$ columns of $\mathbf{A}^{i+1}$. Therefore, for any dimension $q, 1 \leq q \leq p$, the first $q$ columns of $\mathrm{Q}^{i+1}$ can be chosen as a replacement (according to equation 2.59 ) for the parallelotope $\mathrm{J}_{\Phi}^{\mathbf{u}\left(t_{0}+i \Delta t\right)} U_{i}^{q}$ spanned by the first $q$ columns of $\mathbf{A}^{i+1}$. Applying the linearized flow to the parallelotopes $U_{i}^{p}$ as in equation 2.58 therefore corresponds to a matrix multiplication $\mathbf{A}^{i+1}=\mathrm{J}_{\Phi \Delta t}^{\mathbf{u}\left(t_{0}+i \Delta t\right)} \mathbf{Q}^{i}$

The new paralellotopes for the next step given by $\mathbf{Q}^{i+1}$ automatically fulfill $\operatorname{Vol}\left(U_{i+1}^{p}\right)=1$. Since the columns of $\mathbf{Q}^{i+1}$ are orthonormal, the $q$-dimensional volume of the parallelotope given by the first $q$ columns of $\mathbf{A}^{i+1}$ can be calculated using

$$
\operatorname{Vol}\left(\mathrm{J}_{\Phi \Delta t}^{\mathbf{u}\left(t_{0}+i \Delta t\right)} U_{i}^{q}\right)=\prod_{j=1}^{q} R_{j j}^{i+1} .
$$

Putting it all together, we have

$$
\begin{gathered}
\sum_{k=1}^{q} \lambda_{k}=\lim _{t \rightarrow \infty} \frac{1}{t} \ln \operatorname{Vol}\left(J_{\Phi^{t}}^{\mathbf{u}_{0}} U^{q}\right) \stackrel{[2.58}{-} \lim _{m \rightarrow \infty} \frac{1}{m \Delta t} \sum_{l=0}^{m-1} \ln \operatorname{Vol}\left(\mathrm{J}_{\Phi^{\Delta t}}^{\mathbf{u}\left(t_{0}+l \Delta t\right)} U_{l}^{q}\right) \\
\stackrel{2.60)}{=} \lim _{m \rightarrow \infty} \frac{1}{m \Delta t} \sum_{l=0}^{m-1} \sum_{j=1}^{q} \ln R_{j j}^{l+1}=\lim _{m \rightarrow \infty} \frac{1}{m \Delta t} \sum_{l=1}^{m} \sum_{j=1}^{q} \ln R_{j j}^{l} .
\end{gathered}
$$

By subtracting both sides of equation 2.61) for $q=i$ and $q=i-1$, it becomes clear that the Lyapunov exponents are just the time-averaged logarithms of the individual diagonal elements of $\mathbf{R}$ :

$$
\lambda_{i}=\lim _{m \rightarrow \infty} \frac{1}{m \Delta t} \sum_{l=1}^{m} \ln R_{i i}^{l}
$$




\section{Norm-indepencence and covariance}

Lyapunov exponents are independent of the norm (and scalar product) and of the starting point $\mathbf{u}_{0}$ (except for some zero-measure set) on the attractor [111, 176, 175]. This makes them a property of the dynamical system itself, or, more specifically, of one attractor of it, invariant under diffeomorphic coordinate transformations. The same applies to the subspaces $\mathfrak{E}^{(1)} \supset \mathfrak{E}^{(2)} \supset \ldots$, the so-called Oseledec splitting: Naturally, they depend on the coordinates and the reference point $\mathbf{u}_{0}$ on the attractor, but like the corresponding exponents, they are also norm-independent [177]. How are the two Oseledec splittings at two different points on the same trajectory $\mathbf{u}\left(t_{0}+t\right)$ linked to each other? A corollary of equation (2.54) is that

$$
\lim _{t \rightarrow \infty} \frac{1}{t} \ln \left\|J_{\Phi^{t}}^{\mathbf{u}_{0}} \delta \mathbf{u}_{0}\right\| \leq \lambda^{(i)} \quad \Leftrightarrow \quad \delta \mathbf{u}_{0} \in \mathfrak{E}_{\mathbf{u}_{0}}^{(i)}
$$

The suffix $\mathbf{u}_{0}$ was added to $\mathfrak{E}^{(i)}$ in order to indicate the reference point in phase space to which the Oseledec splitting is attached. The above question now reads: what can be said about $\mathfrak{E}_{\mathbf{u}_{1}}^{(i)}$ with $\mathbf{u}_{1}=\mathbf{u}\left(t_{0}+t_{1}\right)$ ? Similarly to equation (2.56), we have for $t>t_{1}$ :

$$
\mathrm{J}_{\Phi^{t}}^{\mathbf{u}_{0}}=\mathrm{J}_{\Phi^{t-t_{1}}}^{\mathbf{u}_{1}} \mathrm{~J}_{\Phi^{t_{1}}}^{\mathbf{u}_{0}}
$$

If we assume that $\mathrm{J}_{\Phi^{t_{1}}}^{\mathbf{u}_{0}}$ is invertible, then substituting equation 2.64 into equation 2.63 ) yields

$$
\lim _{t \rightarrow \infty} \frac{1}{t} \ln \left\|\mathrm{J}_{\Phi^{t-t_{1}}}^{\mathbf{u}_{1}} \mathrm{~J}_{\Phi^{t_{1}}}^{\mathbf{u}_{0}} \delta \mathbf{u}_{0}\right\| \leq \lambda^{(i)} \quad \Leftrightarrow \quad \delta \mathbf{u}_{0} \in \mathfrak{E}_{\mathbf{u}_{0}}^{(i)} \stackrel{\text { invertibility }}{\Leftrightarrow} \quad \mathrm{J}_{\Phi^{t_{1}}}^{\mathbf{u}_{0}} \delta \mathbf{u}_{0} \in \mathrm{J}_{\Phi^{t_{1}}}^{\mathbf{u}_{0}} \mathfrak{E}_{\mathbf{u}_{0}}^{(i)} .
$$

As $J_{\Phi^{t_{1}}}^{\mathbf{u}_{0}}$ is invertible, i.e. bijective, $\mathrm{J}_{\Phi^{t_{1}}}^{\mathbf{u}_{0}} \delta \mathbf{u}_{0}$ can be an arbitrary vector within $\mathrm{J}_{\Phi^{t_{1}}}^{\mathbf{u}_{0}} \mathfrak{E}_{\mathbf{u}_{0}}^{(i)}$. Defining $\delta \mathbf{u}_{1}:=J_{\Phi^{t_{1}}}^{\mathbf{u}_{0}} \delta \mathbf{u}_{0}$, we thus have

$$
\lim _{t \rightarrow \infty} \frac{1}{t} \ln \left\|\mathrm{J}_{\Phi^{t-t_{1}}}^{\mathbf{u}_{1}} \delta \mathbf{u}_{1}\right\| \leq \lambda^{(i)} \quad \Leftrightarrow \quad \delta \mathbf{u}_{1} \in \mathrm{J}_{\Phi^{t_{1}}}^{\mathbf{u}_{0}} \mathfrak{E}_{\mathbf{u}_{0}}^{(i)},
$$

i.e. $J_{\Phi^{t_{1}}}^{\mathbf{u}_{0}} \mathfrak{E}_{\mathbf{u}_{0}}^{(i)}$ is actually $\mathfrak{E}_{\mathbf{u}_{1}}^{(i)}$. In general, the subspaces are thus covariant with the linearized dynamics [175]:

$$
\mathfrak{E}_{\mathbf{u}\left(t_{0}+t\right)}^{(i)}=J_{\Phi^{t}}^{\mathbf{u}_{0}} \mathfrak{E}_{\mathbf{u}_{0}}^{(i)}
$$

\section{Lyapunov vectors}

Assuming again invertibility of the linearized flow, everything said so far for $\mathrm{J}_{\Phi^{t}}^{\mathbf{u}_{0}}$ can likewise be defined for $\left(\mathrm{J}_{\Phi^{t}}^{\mathbf{u}\left(t_{0}-t\right)}\right)^{-1}$ which is the flow mapping a vector in tangent space from time $t_{0}$ back to $t_{0}-t$. If we use the notation ${ }^{-} X$ for the definition of any variable $X$ corresponding to this inverted flow and number Lyapunov exponents and eigenspaces in reverse order, i.e. ${ }^{-} \lambda_{i}{ }^{-} \lambda_{j}$ for $i<j$, equation 2.53 implies

$$
\lambda_{i}=-{ }^{-} \lambda_{i}, \quad 1 \leq i \leq n,
$$


as, due to OsELEDEC's theorem, the Lyapunov exponents do not depend on the starting point on the attractor, and the inverted matrix has reciprocal eigenvalues. Furthermore, we read off equation (2.54):

$$
{ }^{-} \lambda^{(i)}=\lim _{t \rightarrow \infty} \frac{1}{t} \ln \left\|\left(\mathrm{J}_{\Phi^{t}}^{\mathbf{u}\left(t_{0}-t\right)}\right)^{-1} \delta \mathbf{u}_{0}\right\| \quad \delta \mathbf{u}_{0} \in{ }^{-} \mathfrak{E}_{\mathbf{u}_{0}}^{(i)} \backslash \mathfrak{E}_{\mathbf{u}_{0}}^{(i-1)} \subset \mathbb{R}^{n}
$$

${ }^{-} \mathfrak{E}_{\mathbf{u}_{0}}^{(i)}$ is the subspace of $\mathbb{R}^{n}$ which is spanned by eigenspaces for which ${ }^{-} \lambda \leq^{-} \lambda^{(i)}$ :

$$
-\mathfrak{E}_{\mathbf{u}_{0}}^{(i)}=\operatorname{span}\left({ }^{-} E_{\mathbf{u}_{0}}^{(1)},{ }^{-} E_{\mathbf{u}_{0}}^{(2)}, \ldots,{ }^{-} E_{\mathbf{u}_{0}}^{(i)}\right)
$$

and for consistency we define $-\mathfrak{E}_{\mathbf{u}_{0}}^{(0)}:=\{0\}$. An intuitive interpretation of equation 2.69 is: if initialized sufficiently far in the past, perturbations growing like $-{ }^{-} \lambda^{(i)}=\lambda^{(i)}$ (forward in time) will end up in ${ }^{-} \mathfrak{E}_{\mathbf{u}_{0}}^{(i)} \backslash-\mathfrak{E}_{\mathbf{u}_{0}}^{(i-1)}$ at $t_{0}$. Equation 2.54 therefore describes the set of initial conditions which grow with a specific rate, whereas equation 2.69 determines the images of this set under the linearized flow. In analogy to equation $(2.63),-\mathfrak{E}_{\mathbf{u}_{0}}^{(i)}$ is the subspace of vectors that grow backward in time with a rate $\leq^{-} \lambda^{(i)}$ or equivalently with a rate $\geq \lambda^{(i)}$ forward in time:

$$
\lim _{t \rightarrow \infty}-\frac{1}{t} \ln \left\|\left(J_{\Phi^{t}}^{\mathbf{u}\left(t_{0}-t\right)}\right)^{-1} \delta \mathbf{u}_{0}\right\| \geq \lambda^{(i)} \quad \Leftrightarrow \quad \delta \mathbf{u}_{0} \in{ }^{-} \mathfrak{E}_{\mathbf{u}_{0}}^{(i)}
$$

Like the $\mathfrak{E}_{\mathbf{u}_{0}}^{(i)}$, the ${ }^{-} \mathfrak{E}_{\mathbf{u}_{0}}^{(i)}$ are thus covariant. Since the eigenspaces ${ }^{-} E_{\mathbf{u}_{0}}^{(i)}$ are orthogonal, we can find an orthonormal basis ${ }^{-} \mathbf{e}_{\mathbf{u}_{0}}^{i}, 1 \leq i \leq n$ of $\mathbb{R}^{n}$ such that

$$
{ }^{-} \mathfrak{E}_{\mathbf{u}_{0}}^{(i)}=\operatorname{span}\left(-\mathbf{e}_{\mathbf{u}_{0}}^{1},{ }^{-} \mathbf{e}_{\mathbf{u}_{0}}^{2}, \ldots,{ }^{-} \mathbf{e}_{\mathbf{u}_{0}}^{d}\right), \quad d=\operatorname{dim}^{-} \mathfrak{E}_{\mathbf{u}_{0}}^{(i)}, \quad 1 \leq i \leq \tilde{n} .
$$

This basis is unique apart from the choice of an arbitrary orthonormal basis within the eigenspaces with $\operatorname{dim}^{-} E_{\mathbf{u}_{0}}^{(i)}>1$. If a basis fulfills this requirement, the vectors $\mathbf{e}_{\mathbf{u}_{0}}^{i}$ are termed backward Lyapunov vectors [118, because they depend on the action of the flow in the past. Similarly, forward Lyapunov vectors can be defined in the same way as an orthonormal basis of the covariant subspaces $\mathfrak{E}_{\mathbf{u}_{0}}^{(i)}$. Another name for the backward Lyapunov vectors is Gram-Schmidt vectors [118, 114], because they are linked to the matrices $\mathbf{Q}^{i}$ defined above in the following way and the QR-decomposition can be obtained by Gram-Schmidt orthogonalization:

Assume $q=\operatorname{dim} \mathfrak{E}_{\mathbf{u}_{0}}^{\left(q^{*}\right)}$ for some fixed $q^{*}$. The first $q$ columns of the matrix $\mathbf{Q}^{i}$ constructed in section 2.1.8 span a subspace contained in $J_{\Phi^{i \Delta t}}^{\mathbf{u}_{0}}\left(\mathfrak{E}_{\mathbf{u}_{0}}^{(1)} \backslash \mathfrak{E}_{\mathbf{u}_{0}}^{\left(q^{*}+1\right)}\right)$. According to equation $2.54,, \mathfrak{E}_{\mathbf{u}_{0}}^{(1)} \backslash \mathfrak{E}_{\mathbf{u}_{0}}^{\left(q^{*}+1\right)} \subset \mathbb{R}^{n}$ contains all (and only those) vectors that asymptotically grow under the action of the linearized flow with exponential rates $\geq \lambda^{\left(q^{*}\right)}=\lambda_{q}$. Therefore, it follows from equation 2.70 that the subspace spanned by the first $q$ columns of $\mathbf{Q}^{i}$ tends towards $-\mathfrak{E}_{\mathbf{u}\left(t_{0}+i \Delta t\right)}^{(i)}$ for large $t$ (or $i$ ). Since the subspace is by construction $q$-dimensional, the first $q$ columns of $\mathbf{Q}^{i}$ thus tend to the backward Lyapunov vectors $-\mathbf{e}_{\mathbf{u}\left(t_{0}+i \Delta t\right)}^{1},{ }^{-} \mathbf{e}_{\mathbf{u}\left(t_{0}+i \Delta t\right)}^{2}, \ldots,{ }^{-} \mathbf{e}_{\mathbf{u}\left(t_{0}+i \Delta t\right)}^{q}$. As mentioned above, once converged to the corresponding backward eigenspaces, they are unique except for the freedom of choosing arbitrary orthogonal vectors within the eigenspaces of dimensionality greater than 1 . For those cases, the backward Lyapunov vectors one obtains depend on the initially chosen vectors spanning the $q$-dimensional subspace of $\mathfrak{E}_{\mathbf{u}_{0}}^{(1)} \backslash \mathfrak{E}_{\mathbf{u}_{0}}^{\left(q^{*}+1\right)}$. 
The eigenspaces ${ }^{-} E^{(i)}$ themselves are not covariant, because the ${ }^{-} E^{(i)}$ are by construction orthogonal, but the linearized flow is not, in general, angle preserving. This implies that the Gram-Schmidt vectors, are not covariant, either. Obviously, since orthogonality is defined via a norm, they are not norm-independent. However, there is a set of covariant, norm-independent vectors spanning the same covariant subspaces ${ }^{-\mathfrak{E}_{\mathbf{u}\left(t_{0}+i \Delta t\right)}^{(i)}}$, which can be obtained from sets of forward and backward Lyapunov vectors [114, 178, 118]. Consequently they are know as covariant Lyapunov vectors. Despite their recent popularity [114, 115, 116, 117, 118, 119], some authors noticed advantages of the Gram-Schmidt Lyapunov vectors, namely their directionality with respect to time [119]: by construction, they only depend on the past history of the trajectory (cf. equation (2.69)), making them a more "causal" object than the covariant Lyapunov vectors which are invariant under time reversal.

In section 3.1, Lyapunov exponents and Gram-Schmidt vectors will be used to characterize activation patterns in excitable media. The numerical implementation for this specific type of system and its compatibility to the abstract dynamical system of equation 2.46 considered in this section will be outlined in section 2.2.6. 


\subsection{Numerical implementation}

\subsubsection{Time stepping scheme}

Simulations of cardiac tissue using mathematical models as described in sections 2.1.3 and 2.1.6 require the numerical solution of PDEs or, more specifically, reaction-diffusion equations (RDEs). These are of the form of equation 2.13) (page 33). All numerical results in this thesis are based on the numerical procedure of finite differences: Let

$$
\begin{aligned}
\frac{\partial u}{\partial t} & =f(u, \mathbf{h})+\mathcal{L}[u] \\
\frac{\partial \mathbf{h}}{\partial t} & =\mathbf{H}(u, \mathbf{h})
\end{aligned}
$$

be the prototypical RDE that is to be solved. $u(\mathbf{x}, t)$ is the solution for the diffusing variable defined on some domain $\mathcal{D} \times\left[0, T\left[\subset \mathbb{R}^{d} \times \mathbb{R}\right.\right.$ with $d \in\{1,2,3\} . f: \mathbb{R}^{n} \rightarrow \mathbb{R}$ is the local reaction term and $\mathcal{L}$ is the diffusion term (a linear operator) which acts spatially on $u$. $\mathbf{h}$ denotes the vector of local variables and $\mathbf{H}$ defines the corresponding dynamics, as in, e.g., equation 2.13). All simulations in this thesis are initial value problems, meaning that $u(\mathbf{x}, t=0)$ and $\mathbf{h}(\mathbf{x}, t=0)$ are given and the solution for $t \in] 0, T[$ is to be determined. For this purpose, the solution $(u, \mathbf{h})$ is discretized spatially and temporally, with discretization steps $h_{x}, h_{y}$ and $h_{z}$ in the (at most) three space directions and $\Delta t$ in time. This means that the solution is only known at spatially equidistant nodes. Rectangular computational spatial domains are used, even if the physical domain $\mathcal{D}$ is not rectangular, using the phasefield method introduced in section 2.1.5, whose numerical implementation will be explained in section 2.2.3. If the computational domain has a length $L_{l}$ in direction $l(l \in\{x, y, z\})$, the $l$-position of node number $i$ is defined to be

$$
\pi_{l}(i)=\frac{(1+2 i) h_{l}-L_{l}}{2} \quad i \in \mathbb{N}^{0}, 0 \leq i \leq N_{l}-1
$$

The convention $h_{l}=L_{l} / N_{l}$ is used, where $N_{l}$ is the number of nodes in $l$-direction. In the case of three spatial dimensions, this implies that the computational domain is ]$-L_{x} / 2, L_{x} / 2[\times]-L_{y} / 2, L_{y} / 2[\times]-L_{z} / 2, L_{z} / 2\left[\subset \mathbb{R}^{3}\right.$ and that the nodes are placed at the centers of the computational voxels that are $h_{x} \times h_{y} \times h_{z}$ in size. An exemplary geometrical arrangement for a one-dimensional domain is shown in figure 2.9. Numerically, the solution $u$ thus consists of a finite number of values referred to as $u_{i, j, k}^{\tau}$, where $i, j$ and $k$ denote

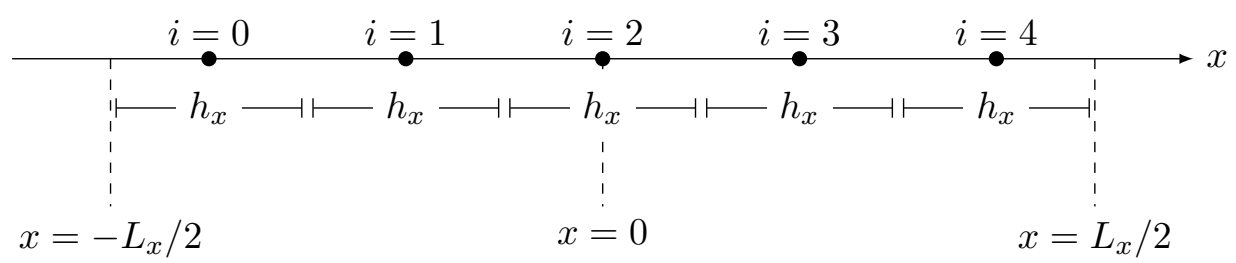

Figure 2.9: Numerical grid points in a one-dimensional domain. The simulation domain is $\mathcal{D}=]-L_{x} / 2, L_{x} / 2\left[\subset \mathbb{R} . N_{x}=5\right.$ numerical nodes are used, whose positions $\pi_{x}(i)$ are indicated by black dots. Each node is centered in a computational box $h_{x}=L_{x} / N_{x}$ in size. 
the three spatial indices and $\tau$ the time index:

$$
\begin{aligned}
u_{i, j, k}^{\tau}:= & u\left(\pi_{x}(i), \pi_{y}(j), \pi_{z}(k), \Delta t \cdot \tau\right) \\
& \text { with } i \in \mathbb{N}^{0}, 0 \leq i \leq N_{l}-1 \quad \forall l \in\{x, y, z\} \\
& \text { and } \tau \in \mathbb{N}^{0}, 0 \leq \tau \leq \frac{T}{\Delta t}
\end{aligned}
$$

The time stepping of consecutive values for all spatial nodes from the initial values $u_{i, j, k}^{0}$ is done using a simple forward Euler or forward-time centered-space (FTCS) scheme:

$$
u_{i, j, k}^{\tau+1}=u_{i, j, k}^{\tau}+\Delta t\left(f\left(u_{i, j, k}^{\tau}\right)+\left(L\left[\mathbf{u}^{\tau}\right]\right)_{i, j, k}\right)
$$

Here, $L$ refers to the approximation of the diffusion operator $\mathcal{L}$ in equation 2.72 . In contrast to $\mathcal{L}, L$ acts on the discretized field $\mathbf{u}^{\tau}$, where this vector quantity contains the set of $u$-values at all numerical grid points at the time step $\tau$. $L$ is usually expressed as a weighted sum of neighboring points, as will be discussed in detail in section 2.2.2, so it acts like a (sparse) matrix on the vector $\mathbf{u}^{\tau} .\left(L\left[\mathbf{u}^{\tau}\right]\right)_{i, j, k}$ refers to the node $(i, j, k)$ of the result of this operator approximation. The time stepping of the local variables (the components of $\mathbf{h}$ ) is done in the same way as in equation (2.77), only without the diffusion term.

\subsubsection{Diffusion Term}

Approximations of the linear operator $\mathcal{L}$ can be obtained in different ways, depending on the specific form of $\mathcal{L}$. They all have in common that the approximation $L$ can usually be written as a convolution of the form:

$$
(\mathcal{L}[u])\left(\pi_{x}\left(k^{*}\right), \pi_{y}\left(l^{*}\right), \pi_{z}\left(m^{*}\right)\right) \approx(L[\mathbf{u}]) k_{k^{*}, l^{*}, m^{*}}=\sum_{k, l, m \in\{-1,0,1\}} \alpha_{k, l, m} \underbrace{u_{k^{*}+k, l^{*}+l, m^{*}+m}}_{=\left(\mathrm{T}_{m}^{z} \mathrm{~T}_{l}^{y} \mathrm{~T}_{k}^{x} u\right)_{k^{*}, l^{*}, m^{*}}} .
$$

The time dependence has been omitted and will be for the rest of this section, as $\mathcal{L}$ and $L$ act solely on the spatial degrees of freedom. Defining a discrete approximation $L$ for $\mathcal{L}$ now reduces to finding a suitable kernel $\alpha$. For this purpose, it is useful to define the translation operator $\mathrm{T}_{n}^{i} u$, which takes the field $u$ and shifts it by a given amount $-n h_{i}$ in the direction $i$, where $i \in x, y, z$, such that

$$
\begin{aligned}
\left(\mathrm{T}_{+1}^{x} u\right)(x, y, z) & =u\left(x+h_{x}, y, z\right) \\
\text { or } \quad\left(\mathrm{T}_{+1}^{x} u\right)_{k, l, m} & =u_{k+1, l, m} .
\end{aligned}
$$

From the definition it follows that translation operators in the same direction follow the summation rule

$$
\mathrm{T}_{n_{1}}^{i} \mathrm{~T}_{n_{2}}^{i} u=\mathrm{T}_{n_{1}+n_{2}}^{i} u
$$


and that they correspond to the identity if the shift is zero, i.e.

$$
\mathrm{T}_{0}^{i} u=u
$$

In the following, $\delta_{i, j}$ denotes the Kronecker delta, which is 1 if and only if $i=j$ and 0 otherwise. The inverse Kronecker delta $\bar{\delta}$ is defined as $\bar{\delta}_{i, j}=1-\delta_{i, j}$.

\section{General Diffusion Tensors}

A discretization for the general form of the diffusion term $\mathcal{L}=\nabla \cdot \underline{\mathbf{D}} \nabla$ with a spacedependent diffusion tensor $\underline{\mathbf{D}}$ can be obtained by approximating all derivatives by centered finite differences, i.e. $\partial_{i} u \approx\left(\mathrm{T}_{\frac{1}{2}}^{i} u-\mathrm{T}_{-\frac{1}{2}}^{i} u\right) / h_{i}$, where $\partial_{i}$ is the spatial derivative in the direction $i$ and $h_{i}$ the corresponding spatial discretization step. In the most general case, $\underline{\mathbf{D}}$ is space-dependent, which means that each component $D_{i j}$ of $\underline{\mathbf{D}}$ is itself a field. For values of $u$ and $D_{i j}$ in between two numerical grid points, the definition

$$
\mathrm{T}_{\frac{\mu}{2}}^{i}:=\frac{\mathrm{T}_{0}^{i}+\mathrm{T}_{\mu}^{i}}{2}
$$

is used, where $\mu \in\{1,-1\}$. In this way, the following expansion for a general diffusion term is obtained:

$$
\begin{aligned}
& \nabla \cdot \underline{\mathbf{D}} \nabla u=\sum_{i \in\{x, y, z\}} \partial_{i} \sum_{j \in\{x, y, z\}} D_{i j} \partial_{j} u \\
& \approx \sum_{i \in\{x, y, z\}} \frac{1}{h_{i}} \partial_{i}\left(\sum_{j \in\{x, y, z\}} D_{i j}\left(\mathrm{~T}_{\frac{1}{2}}^{j} u-\mathrm{T}_{-\frac{1}{2}}^{j} u\right)\right. \\
& \approx \sum_{i=j} \frac{1}{h_{i}^{2}}\left(\mathrm{~T}_{\frac{1}{2}}^{i} D_{i i} \cdot\left(\mathrm{T}_{1}^{i} u-u\right)-\mathrm{T}_{-\frac{1}{2}}^{i} D_{i i} \cdot\left(u-\mathrm{T}_{-1}^{i} u\right)\right) \\
& +\sum_{i \neq j} \frac{1}{h_{i} h_{j}}\left[\mathrm{~T}_{\frac{1}{2}}^{i} D_{i j} \cdot\left(\mathrm{T}_{\frac{1}{2}}^{i} \mathrm{~T}_{\frac{1}{2}}^{j} u-\mathrm{T}_{\frac{1}{2}}^{i} \mathrm{~T}_{-\frac{1}{2}}^{j} u\right)\right. \\
& \left.-\mathrm{T}_{-\frac{1}{2}}^{i} D_{i j} \cdot\left(\mathrm{T}_{-\frac{1}{2}}^{i} \mathrm{~T}_{\frac{1}{2}}^{j} u-\mathrm{T}_{-\frac{1}{2}}^{i} \mathrm{~T}_{-\frac{1}{2}}^{j} u\right)\right] \\
& =\sum_{i=j} \frac{1}{h_{i}^{2}} \sum_{\mu \in\{-1,1\}} \mathrm{T}_{\frac{\mu}{2}}^{i} D_{i i} \cdot\left(\mathrm{T}_{\mu}^{i} u-u\right) \\
& +\sum_{i \neq j} \frac{1}{4 h_{i} h_{j}}[ \\
& \mathrm{T}_{\frac{1}{2}}^{i} D_{i j} \cdot\left(\mathrm{T}_{1}^{i} \mathrm{~T}_{1}^{j} u+\mathrm{T}_{1}^{j} u-\mathrm{T}_{1}^{i} \mathrm{~T}_{-1}^{j} u-\mathrm{T}_{-1}^{j} u\right) \\
& \left.-\mathrm{T}_{-\frac{1}{2}}^{i} D_{i j} \cdot\left(\mathrm{T}_{-1}^{i} \mathrm{~T}_{1}^{j} u+\mathrm{T}_{1}^{j} u-\mathrm{T}_{-1}^{i} \mathrm{~T}_{-1}^{j} u-\mathrm{T}_{-1}^{j} u\right)\right] \\
& =\sum_{i=j} \frac{1}{h_{i}^{2}} \sum_{\lambda \in\{-1,1\}} \sum_{\mu \in\{-1,1\}} \lambda \mu \mathrm{T}_{\frac{\mu}{2}}^{i} D_{i i} \cdot\left(\delta_{\mu, \lambda} \mathrm{T}_{\mu}^{i} u+\bar{\delta}_{\mu, \lambda} u\right) \\
& +\sum_{i \neq j} \frac{1}{4 h_{i} h_{j}} \sum_{\lambda \in\{-1,1\}} \sum_{\mu \in\{-1,1\}} \lambda \mu \mathrm{T}_{\frac{\mu}{2}}^{i} D_{i j} \cdot\left(\mathrm{T}_{\mu}^{i} \mathrm{~T}_{\lambda}^{j} u+\mathrm{T}_{\lambda}^{j} u\right)
\end{aligned}
$$




$$
\begin{array}{r}
=\sum_{\substack{i, j \in\{x, y, z\} \\
\lambda, \mu \in\{-1,1\}}} \frac{\lambda \mu \mathrm{T}_{\frac{\mu}{2}}^{i} D_{i j}}{h_{i} h_{j}} \cdot\left[\delta_{i, j}\left(\delta_{\mu, \lambda} \mathrm{T}_{\mu}^{i} u+\bar{\delta}_{\mu, \lambda} u\right)\right. \\
\left.+\frac{\bar{\delta}_{i, j}}{4}\left(\mathrm{~T}_{\mu}^{i} \mathrm{~T}_{\lambda}^{j} u+\mathrm{T}_{\lambda}^{j} u\right)\right]
\end{array}
$$

At most two shift operators are applied to $u$ in equation (2.84), such that by comparison with (2.78), $\alpha_{k, l, m}=0$ for $|k|=|l|=|m|=1$. The remaining elements of the kernel $\alpha_{k, l, m}$ can be calculated directly by iterating through the kernel as suggested by equation 2.84 (see listing 2.1).

\section{Listing 2.1: Calculation of Laplace kernel for a space dependent diffusion tensor. The ker- nel elements $\alpha_{k, l, m}$ are constructed for one specific point in the computational domain and saved to the array kernel. The scheme follows exactly equation (2.84), see also comments in the code.}

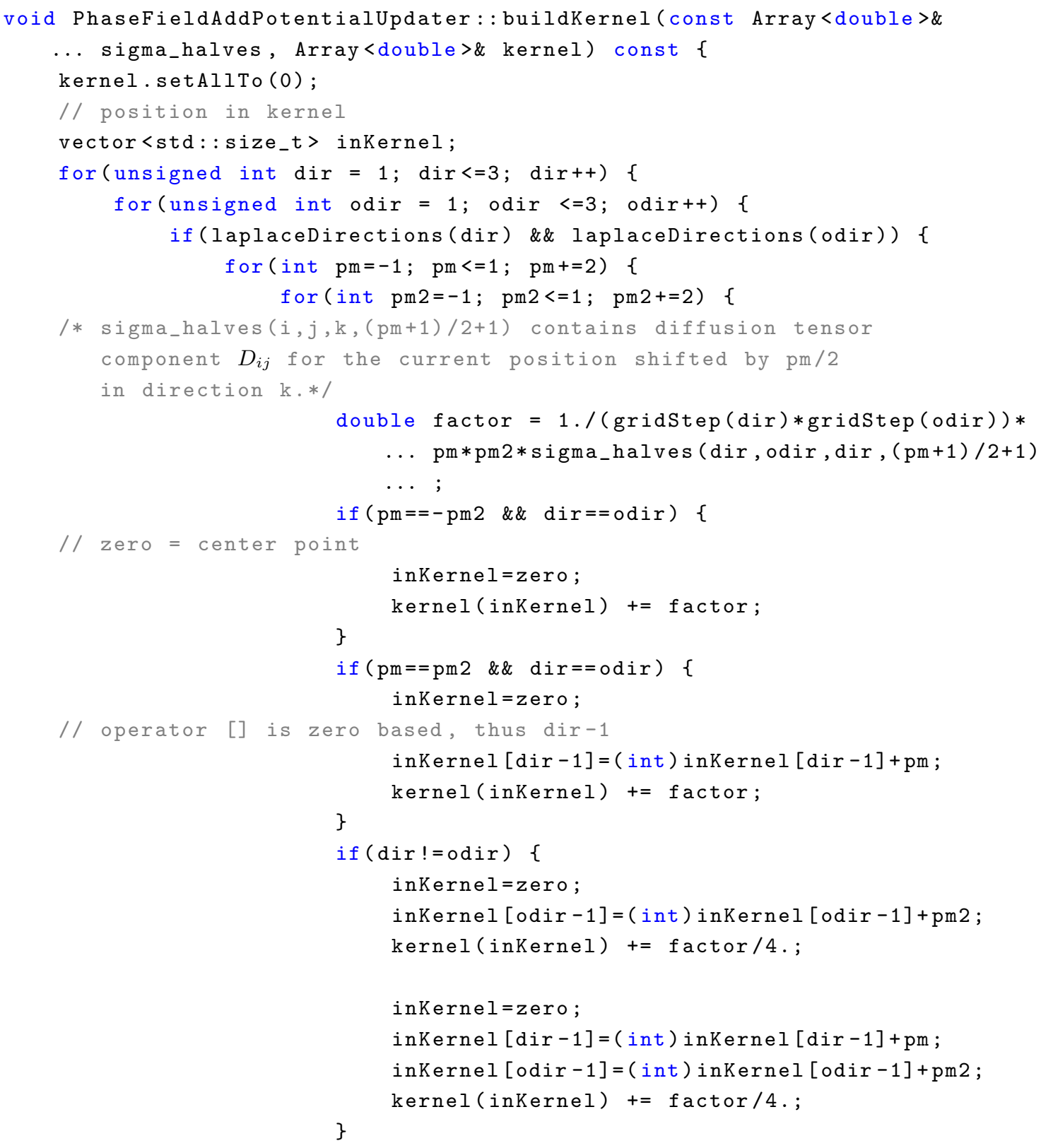




$3+3 \quad\}^{3}$

\section{Homogeneous, isotropic diffusion}

For spatially constant and isotropic diffusion tensors $\underline{\mathbf{D}}=D \mathrm{Id}$, i.e. $\mathcal{L}=\nabla \cdot \underline{\mathbf{D}} \nabla=D \nabla^{2}$, throughout the computational domain, and equal discretization steps $h_{x}=h_{y}=h_{z}=h$ in all directions, the kernel elements $\alpha_{k, l, m}$ become symmetric in the sense that

$$
\left|k_{1}\right|+\left|l_{1}\right|+\left|m_{1}\right|=\left|k_{2}\right|+\left|l_{2}\right|+\left|m_{2}\right| \quad \Rightarrow \quad \alpha_{k_{1}, l_{1}, m_{1}}=\alpha_{k_{2}, l_{2}, m_{2}},
$$

meaning that only the number of non-zero indices $k, l, m$ determines the value of the kernel element. From equation (2.84), the following values are obtained:

$$
\alpha_{k, l, m}=\frac{D}{h^{2}} \cdot \begin{cases}-6 & \text { for } k=l=m=0 \\ 1 & \text { for }|k|+|l|+|m|=1 \\ 0 & \text { for }|k|+|l|+|m|=2\end{cases}
$$

Under the assumption $\mathrm{T}_{1}^{z} u=\mathrm{T}_{-1}^{z} u=u$, all derivatives in the $z$-direction vanish and the dynamics for a two-dimensional medium is obtained. Thus, a two-dimensional kernel $\alpha_{k, l}^{2 \mathrm{~d}}$ can be obtained by setting $\alpha_{k, l}^{2 \mathrm{~d}}=\sum_{m=-1,0,1} \alpha_{k, l, m}$ for all $k, l$. In this case, the kernel reduces to the usual five-point stencil (see equation 12.5.1 in reference [179]) for the Laplacian $D \nabla^{2}$ :

$$
\left(\alpha_{k, l}^{2 \mathrm{~d}}\right)_{\substack{k=-1,0,1 \\
l=-1,0,1}}=\frac{D}{h^{2}}\left(\begin{array}{ccc}
0 & 1 & 0 \\
1 & -4 & 1 \\
0 & 1 & 0
\end{array}\right) .
$$

A Taylor expansion of $u(x, y)$ reveals that the approximation ${ }^{2 \mathrm{~d}} L$ for the Laplacian defined by this kernel (and also by the $\alpha_{k, l, m}$ above) is second order accurate, i.e. $\nabla^{2} u={ }^{2 \mathrm{~d}} L u+$ $\mathcal{O}\left(h^{2}\right)$, which is to be expected because it originates from the second-order finite difference discretization in section 2.2 .2 .

If isotropy and a spatially constant diffusion tensor $\underline{\mathbf{D}}$ are present, instead of $\alpha$ above, the following kernel $\tilde{\alpha}$ can be used:

$$
\tilde{\alpha}_{k, l, m}=\frac{D}{30 h^{2}} \cdot \begin{cases}-128 & \text { for } k=l=m=0 \\ 14 & \text { for }|k|+|l|+|m|=1 \\ 3 & \text { for }|k|+|l|+|m|=2 \\ 1 & \text { for }|k|+|l|+|m|=3\end{cases}
$$

Again, it is defined for $-1 \leq k, l, m \leq 1$. A Taylor expansion shows that this kernel is second-order accurate and thus might not seem to have any advantage over $\alpha$. However, it preserves the rotational symmetry of the Laplacian much more accurate, which results 
in reduced grid-induced distortions of the dynamics at a fixed spatial discretization length $h$. Using the same method as above, the two-dimensional version of this stencil can be calculated as

$$
\left(\tilde{\alpha}_{k, l}^{2 \mathrm{~d}}\right)_{\substack{k=-1,0,1 \\
l=-1,0,1}}=\frac{D}{6 h^{2}}\left(\begin{array}{ccc}
1 & 4 & 1 \\
4 & -20 & 4 \\
1 & 4 & 1
\end{array}\right),
$$

the so-called nine-point stencil (see equation 12.5.4 in reference [179]).9

\subsubsection{Boundary conditions}

\section{Rectangular domains}

For numerical grid points on the boundary, the convolution (2.78) contains neighbor points outside the actual computational domain, whose values are not defined. When $\underline{\mathbf{D}}$ is spatially constant and isotropic, these can be used to implement the desired boundary condition in conjunction with the simple kernels introduced in section 2.2.2. For a rectangular grid, the normal fluxes $\mathbf{n} \cdot \underline{\mathbf{D}} \nabla u$ at the boundaries are just derivatives of $u$ along grid directions. For example, the flux at the boundary $x=\pi_{x}(0)$ can be approximated by a simple centered finite difference:

$$
\left.\mathbf{n} \cdot \underline{\mathbf{D}} \nabla u\right|_{x=\pi_{x}(0)}=\left.D_{1,1} \frac{\partial u}{\partial x}\right|_{x=\pi_{x}(0)} \approx D_{1,1} \frac{u_{1, j, k}-u_{-1, j, k}}{2 h_{x}}
$$

For no-flux boundary conditions, this term has to vanish, so defining $u_{-1, j, k}:=u_{1, j, k}$ yields the desired result. For periodic boundary conditions, the numerical point just outside the domain is actually identical to the one on the opposing side. Therefore the definition in this case reads $u_{-1, j, k}:=u_{N_{x}-1, j, k}$. The same method is applied to the boundary at $x=\pi_{x}\left(N_{x}-1\right)$ and for the other space directions.

\section{Domains of arbitrary shape}

If $\mathcal{D}$ is non-rectangular, the phase-field method introduced in section 2.1.5 is employed. A large enough computational domain $\mathcal{D}_{c}$ is chosen that contains $\mathcal{D}$. Then, for no-flux, technically, the replacement 2.19) is carried out by letting

$$
\underline{\mathbf{D}}=\phi \underline{\mathbf{D}}_{\text {phys }},
$$

where $\underline{\mathbf{D}}_{\text {phys }}$ is the physical diffusion tensor and $\phi$ is the phase field introduced in section 2.1.5. describing the shape of the tissue domain $\mathcal{D}$. Even if $\underline{\mathbf{D}}_{\text {phys }}$ is spatially constant, a space dependence of $\underline{\mathbf{D}}$ is introduced by the phase field. Thus, the numerical scheme developed in section 2.2 .2 is applied. After calculating the local kernel elements $\alpha_{k, l, m}$ according to equation (2.84), all weights have to be divided by the local value of the phase field $\phi(x)$ in order to account for the additional factor $1 / \phi$ in equation 2.19).

\footnotetext{
${ }^{9}$ The fourth-order accuracy of the nine-point stencil sometimes referred to is only present under special circumstances (for example, for the solution of the Poisson equation), because the fourth-order error has a special form that can be corrected for in those cases.
} 
Since $\phi$ and $\underline{\mathbf{D}}_{\text {phys }}$ are independent of time, this kernel calculation has to be carried out only once at the beginning of the simulation, allowing for an efficient implementation of no-flux boundary conditions for arbitrarily shaped tissue domains $\mathcal{D}$.

To enforce the more general boundary condition of the type of equation $(2.36)$, the phasefield replacement (2.37) is rewritten as

$$
\frac{1}{\phi} \nabla \cdot(\phi(\underline{\mathbf{D}} \nabla u-\mathbf{E}))=\frac{1}{\phi} \nabla \cdot\left(\phi \underline{\mathbf{D}} \nabla u+\underline{\tilde{\mathbf{D}}} \nabla v_{E}\right)=\frac{1}{\phi} \nabla \cdot(\phi \underline{\mathbf{D}} \nabla u)+\frac{1}{\phi} \nabla \cdot\left(\phi \underline{\tilde{\mathbf{D}}} \nabla v_{E}\right)
$$

where $v_{E}$ is chosen such that $\mathbf{E}=-\underline{\tilde{\mathbf{D}}} \nabla v_{E}$. If the outside potential $v_{o}$ in equation 2.16 (page 33) is assumed to have a constant gradient, $v_{E}$ is identical to $v_{o}$, with an appropriately chosen $\underline{\tilde{\mathbf{D}}}$. Substituting the right hand side of equation 2.92 for $\mathcal{L}[u]$ in equation 2.72 , the numerical scheme introduced in section 2.2 .2 can be applied to both diffusion terms, as explained above for the no-flux case. Splitting up the two terms as in equation 2.92 is particularly convenient, if the assumed potential $v_{E}$ is independent of time or merely multiplied by a time-dependent prefactor. In this case, the entire second term has to be calculated only once at the beginning of the simulation. The result of this calculation (a field of the size of the computational domain $\mathcal{D}_{c}$ ) is then multiplied by $\Delta t$ and possibly the time-dependent factor, before being added to equation (2.77).

As mentioned in [134], the results of a numerical simulation with the phase-field method change only insignificantly if numerical calculations are performed solely for grid points for which $\phi_{i, j, k}>\phi_{\text {cut-off }}$ with $\phi_{\text {cut-off }} \ll 1$. The spatial domain in which $\phi>\phi_{\text {cut-off }}$ holds corresponds to a slightly enlarged tissue domain $\mathcal{D}$ within the auxiliary domain $\mathcal{D}_{c}$. As explained in section 2.1.5, the pseudo-advection term in the phase-field method leads to a relative independence of the dynamics in $\mathcal{D}$ from the boundary condition imposed at $\partial \mathcal{D}_{c}$. In essence, skipping the numerical update step for the values of computational points for which $\phi_{i, j, k}<\phi_{\text {cut-off }}$ corresponds to an artificial Dirichlet boundary condition imposed at the boundary of the enlarged tissue domain (which would also be a valid choice for $\mathcal{D}_{c}$, but usually rectangular domains are used). Therefore, the validity of this approach follows from the line of thoughts in section 2.1.5. In all simulations throughout this thesis $\phi_{\text {cut-off }}=10^{-5}$ is used.

\subsubsection{Stability considerations}

For the assessment of numerical stability, the nature of the different terms of the RDE has to be taken into account. One limitation arises from the typically large upstroke velocity of the membrane potential for a beginning excitation in cardiac models, which is a result of the local reaction term $f$ in equation 2.72 or correspondingly $-I_{\text {ion }} / C_{m}$ in equation 2.13). A name often used to describe problems with strongly differing time scales is stiff systems. To avoid instabilities due to this stiffness, the time step for an explicit Euler scheme like equation (2.77) has to be considerably smaller than the time scale $\tau_{\text {upstroke }}=A_{\mathrm{AP}} / v_{\text {upstroke }}$, where $A_{\mathrm{AP}}$ is the action potential amplitude and $v_{\text {upstroke }}$ is the maximal rate of change during the upstroke. As the typical upstroke time in cardiac cells is on the millisecond order, this restricts $\Delta t$ to the sub-millisecond range for models that work with real units.

Another possible instability arises from the diffusion term. If equation 2.77$)$ is considered 
without the reaction term, for isotropic diffusion $\underline{\mathbf{D}}=D \mathrm{Id}$, the discretized version of a heat equation is obtained. Applying a von Neumann stability analysis to this discretization (using the kernels of equation (2.86) ) leads to a stability condition for the forward Euler scheme, namely

$$
D \frac{\Delta t}{h^{2}} \leq \frac{1}{2 d}
$$

where $d$ is the number of spatial dimensions (equation 19.2.6 in reference [180] generalized to higher dimensions). This is a stringent restriction, since it means that a small $h$ which might be necessary by itself to resolve spatial details of the dynamics automatically require a quadratically smaller $\Delta t$. In three dimensions, if the left hand side of equation (2.93) is kept constant, halving $h$ thus leads to an approximately 32 -fold increase in computational cost, because the number of numerical grid points increases by a factor of eight and $\Delta t$ has to be divided by four.

Both kinds of stability criteria could be relaxed or avoided by using more sophisticated time stepping schemes than FTCS. However, despite providing the desired stability, the additional computational cost - arising, e.g., for implicit methods from solving large systems of linear equations - does not necessarily bring about an equal increase in accuracy. The accurate numerical computation of the solution even on small spatial scales is desirable in particular for models of cardiac tissue, because the interaction of diffusion and rapid upstrokes on these scales is essential for the propagation of wave fronts. Thus, reducing $h$ without reducing $\Delta t$ to faithfully model the physics of the additional small scales is usually leading nowhere, even if stability is guaranteed by the numerical method. Nevertheless, computation time and memory consumption can be reduced to some extent by using alternative numerical schemes [181] (see [32] for a recent summary of widely-used techniques). However, considering the additional implementation and debugging costs in addition to the arguments above, an explicit FTCS scheme is a less unfavorable numerical scheme for this type of problem than it may seem at first sight.

It should be noted that the stability criterion 2.93) applies also to spatially varying diffusion tensors $\underline{\mathbf{D}}$, as long as they vary slowly. In this case, the von Neumann stability analysis is thought to be carried out locally. This condition is certainly not fulfilled, when the phase-field method introduced in section 2.1.5 is employed. Here, the diffusion tensor is multiplied by $\phi$, a quantity which decays rapidly at the physical system boundary. Far away from the physical domain, it decays exponentially with a decay length $\approx \xi$ comparable to the grid spacing $h$. The normal derivative of the phase field is therefore $|\mathrm{d} \ln \phi / \mathrm{d} n| \lesssim 1 / \xi$. According to equation $(2.29)$, this leads to an advection-like term with a velocity of $|v| \lesssim D / \xi$, if the original diffusion tensor is again assumed to be isotropic with $\underline{\mathbf{D}}=D \mathrm{Id}$. For any explicit time stepping scheme of an advection equation, the CourantFriedrichs-Lewy criterion for numerical stability applies (equation 19.1.17 in reference [180]):

$$
1 \geq \frac{|v| \Delta t}{h} \gtrsim D \frac{\Delta t}{\xi h}
$$

The values for $\xi$ used in this thesis range between $h / 2$ and $h$ such that equation (2.94) is not a more stringent restriction on $\Delta t$ than equation (2.93). Of course, this calculation is 
only an approximation and equation (2.94) should be taken as an order-of-magnitude estimation. Also, additional instabilities could arise from the steep increase of the advection velocity near the boundary of the physical domain, and the behavior at some intermediate distance from the boundary might also differ from the simple exponential decay due to the geometry of the boundary. However, equation (2.94) shows that, at zeroth order, there is no reason why the phase-field method should destabilize the numerical solution. In reality, test simulations show that numerical simulations using the phase-field method are mostly stable provided they were without the phase field ( $\phi=1$ everywhere). Only sometimes, it is necessary to reduce $\Delta t$ slightly, never leaving, however, the order of magnitude suggested by equation (2.93).

\subsubsection{Spiral tip detection}

The tips of spiral waves in two-dimensional simulations of the Barkley model are localized by determining the topological charge of small test domains within the simulation domain according to equation 2.45. To numerically calculate the integral along a closed curve, the method of IYER and Gray [170] is used:

$$
2 \pi n_{i, j}^{\text {top }}=\oint_{\mathcal{C}_{i, j}} \nabla \theta \mathrm{d} \mathbf{l} \approx \sum_{m=2}^{n} \operatorname{diff}\left(\theta_{i+k_{m}, j+l_{m}}, \theta_{i+k_{m-1}, j+l_{m-1}}\right)
$$

The offsets $k_{1}, \ldots, k_{n}$ and $l_{1}, \ldots, l_{n}$ parameterize a path $\mathcal{C}_{i, j}$ around the point $(i, j)$ in numerical coordinates. The function diff yields the phase difference between two points. As explained in section 2.1.7, for the local phase differences, the branch of the arctan2 function has to be chosen such that there is no phase jump between the two points whose phase difference is to be determined. A unique difference is obtained by assuming that the phase difference is always smaller in magnitude than $\pi$ :

$$
\operatorname{diff}(x, y):=\bmod (x-y+\pi, 2 \pi)-\pi \quad \in[-\pi, \pi[
$$

The mod function is defined such that it returns positive values and thus the result of diff ends up in the desired range. As in reference [170], an eight-point integration path is chosen:

$$
\left(\begin{array}{c}
k_{1}, \ldots, k_{n} \\
l_{1}, \ldots, l_{n}
\end{array}\right)=\left(\begin{array}{ccccccccc}
1 & 1 & 0 & -1 & -1 & -1 & 0 & 1 & 1 \\
0 & 1 & 1 & 1 & 0 & -1 & -1 & -1 & 0
\end{array}\right)
$$

On the one hand, this integration path is small enough to minimize the risk of enclosing multiple phase singularities, such that isolated phase singularities can be detected. On the other hand, it contains enough sampling points to avoid large phase jumps on the integration path (after all, the phase differences have to add up to $2 \pi$ if a phase singularity is enclosed). Such large jumps of $\theta$ could cause the definition equation (2.96) to miss full turns of the phase. Phase singularities and their corresponding spiral tips can then be detected by locating the points $(i, j)$ for which $n_{i, j}^{\text {top }}= \pm 1$ in equation 2.95. Usually, four adjacent numerical points (a $2 h \times 2 h$ region) are detected as possible candidates for the location of a phase singularity, since the integration path encloses a region which is 
$2 h \times 2 h$ in size. Within this thesis, the position of the phase singularity is then defined to be in the center of this $2 h \times 2 h$ region.

\subsubsection{Lyapunov exponents and vectors}

\section{Linearization of an extended system}

In section 2.1.8, the theory of Lyapunov stability analysis and the standard mathematical strategy for its implementation were summarized for a general dynamical system like equation (2.46). In this thesis, the analysis is applied to extended systems of the type of equation (2.72). Obviously, in the framework of section 2.1.8, it is not possible to include differential operators directly on the right hand side of equation (2.46). However, this is possible for the semi-discretized form of equation 2.72

$$
\begin{aligned}
\frac{\mathrm{d} u_{i, j, k}}{\mathrm{~d} t}=f\left(u_{i, j, k}, h_{1 i, j, k}, h_{2 i, j, k}, \ldots\right)+(L[\mathbf{u}])_{i, j, k} & \\
\frac{\mathrm{d} h_{1 i, j, k}}{\mathrm{~d} t}=H_{1}\left(u_{i, j, k}, h_{1 i, j, k}, h_{2 i, j, k}, \ldots\right) & \\
\frac{\mathrm{d} h_{2 i, j, k}}{\mathrm{~d} t}=H_{2}\left(u_{i, j, k}, h_{1 i, j, k}, h_{2 i, j, k}, \ldots\right) & \forall i, j, k
\end{aligned}
$$

where the vector quantity $\mathbf{u}$ again contains the values of $u$ at all numerical nodes by defining a fixed mapping of $(i, j, k)$ to a linear index. Similar vectors with the same mapping can be defined for each of the local variables $h_{i}$, which were summarized as $\mathbf{h}=\left(h_{1}, h_{2}, \ldots\right)$ in, e.g., equation (2.4). For clarity, they have been separated here. As the approximation $L$ for the differential operator $\mathcal{L}$ is expressed as a convolution of the form 2.78), equation 2.98 represents a system of coupled ODEs, together with the boundary conditions introduced in section 2.2.3. Let the set of $u_{i, j, k}(t), h_{1 i, j, k}(t), h_{2 i, j, k}(t)$, $\ldots$ for all $i, j, k$ be a solution of this dynamical system. Then, the linearized dynamics of equation 2.49 (again, with all vector components given separately) has the following structure:

$$
\begin{array}{rlr}
\frac{\mathrm{d} \delta u_{i, j, k}}{\mathrm{~d} t} & =\mathrm{J}_{f}^{\left(u_{i, j, k}, h_{1 i, j, k}, h_{2 i, j, k}, \ldots\right)} \delta u_{i, j, k}+(L[\delta \mathbf{u}])_{i, j, k} & \\
\frac{\mathrm{d} \delta h_{1 i, j, k}}{\mathrm{~d} t} & =\mathrm{J}_{H_{1}}^{\left(u_{i, j, k}, h_{1 i, j, k}, h_{2 i, j, k}, \ldots\right)} \delta h_{1 i, j, k} & \\
\frac{\mathrm{d} \delta h_{2 i, j, k}}{\mathrm{~d} t} & =\mathrm{J}_{H_{2}}^{\left(u_{i, j, k}, h_{1 i, j, k}, h_{2 i, j, k}, \ldots\right)} \delta h_{2 i, j, k} &
\end{array}
$$

It is worth noting that the Jacobian matrices for $f, H_{1}, H_{2}, \ldots$ are indeed only the lowdimensional matrices for the local dynamics. Just like the functions $f, H_{1}, H_{2}, \ldots$, they are identical for all numerical grid points of the discretization. For the models used in this thesis, they are easily calculated by hand for arbitrary values of $u, h_{1}, h_{2}, \ldots$ and can be found in appendix A. To guarantee differentiability, the smooth Fenton-Karma model introduced in section 2.1.6 is used instead of the original model. The discretization $L$ of the operator $\mathcal{L}$ introducing the spatial coupling is linear (as is the original operator). 
Thus, its Jacobian is the operator itself and its numerical implementation can be used without modification for the evolution of the linearized system.

For periodic or no-flux boundary conditions, this is true even at the boundary: According to the explanations in section 2.2.3, both types of boundary conditions can be implemented by exchanging certain numerical points in the convolution $(2.78)$ for others. This procedure preserves the linearity of the operator (in its discretized form), which is why the linearized operator again is just the operator itself. In fact, this strategy should work just as well for no-flux boundary conditions implemented by the phase field method, although, in this thesis, Lyapunov stability analysis will only be carried out for simple, rectangular geometries. For Dirichlet boundary conditions (not used in this thesis), a similar argument leads to the conclusion that the original boundary condition has to be replaced by a Dirichlet boundary condition with the value 0 everywhere on the boundary for the linearized operator.

A different way of looking at equation $(2.99)$ is to view it as a discretization of the system of partial differential equations

$$
\begin{aligned}
\frac{\partial \delta u}{\partial t} & =\mathrm{J}_{f}^{\left(u, h_{1}, h_{2}, \ldots\right)} \delta u+\mathcal{L}[\delta u] \\
\frac{\partial \delta h_{1}}{\partial t} & =\mathrm{J}_{H_{1}}^{\left(u, h_{1}, h_{2}, \ldots\right)} \delta h_{1} \\
\frac{\partial \delta h_{2}}{\partial t} & =\mathrm{J}_{H_{2}}^{\left(u, h_{1}, h_{2}, \ldots\right)} \delta h_{2}
\end{aligned}
$$

where $\delta u, \delta h_{1}, \delta h_{2}, \ldots$ are now functions of space again. In reference [177], ECKMANN and RUELLE note that Lyapunov stability analysis is possible on function spaces. This is a first indication that the Lyapunov spectra obtained from the linearization of the semidiscretized system equation 2.98 might actually be used to characterize the original reaction-diffusion system itself. This will be investigated in detail in section 3.1 .

\section{Orthonormalization}

Most of the stability analysis will be carried out for two-dimensional systems. Assuming a spatial discretization with $N_{x} N_{y}$ nodes (according to the scheme introduced in section 2.2.1 and a model with one diffusing variable and $N_{v}-1$ local variables, the total number of degrees of freedom of the semi-discretized system (2.98) as well as for the linearized system 2.99) is $N_{\text {dof }}=N_{x} N_{y} N_{v}$. Typical numbers are $N_{x}=N_{y}=150$ and, e.g. for the Barkley model, $N_{v}=2$, resulting in $N_{\text {dof }}=45000$. To calculate the $N_{\text {lyap }}$ leading Lyapunov exponents, $N_{\text {lyap }}$ copies of the system (2.99) have to be integrated alongside the actual dynamics. Hence, the matrix $\mathbf{A}$ from section 2.1.8 containing one perturbation in each column is a $N_{\text {dof }} \times N_{\text {lyap }}$-matrix. The perturbations are initialized randomly and orthonormalized at regular intervals. According to section 2.1.8, they converge to the $N_{\text {lyap }}$ backward Lyapunov vectors corresponding to the $N_{\text {lyap }}$ most expanding directions. The accuracy of the Lyapunov exponents calculated as in equation 2.62) (page 53) can be improved by averaging the growth rates only after this alignment of the perturbations has taken place. The amount of time the perturbations were allowed to align for will be indicated along the with the results of the simulations in section 3.1 . 
Because of the large number of rows, the Gram-Schmidt orthogonalization procedure used to compute the QR-decomposition of $\mathbf{A}$ can be parallelized efficiently. The scalar products of different columns $\mathbf{A}$ with the columns of $\mathbf{Q}$ that have already been obtained are calculated in separate threads. Moreover, when the results of this calculation are used to subtract the projections of the preceding columns from the current column, work in different blocks of rows is separated between threads.

In addition, the orthonormalization interval $\Delta t$ in section 2.1 .8 does not necessarily have to equal the numerical time step $\Delta t$ in section 2.2.1. In fact, the orthonormalization interval is the result of an arbitrary splitting of the linearized flow as equations $(2.56)$ and (2.58) indicate. The purpose of this splitting is to prevent the volume spanned by the perturbations from collapsing, which may happen if two of the perturbations become linearly dependent due to finite-precision arithmetics. Choosing a larger orthonormalization interval leads to a considerable gain in computation time, since the costly QR-decomposition has to be carried out less often. To check the validity of the results, the resulting Lyapunov exponents are compared with those from simulations for which the orthonormalization procedure was carried out in each time step. For all results presented in this thesis, the results were ensured to be numerically indistinguishable with regard to the criterion explained below.

\section{Convergence measure}

The result of the Lyapunov stability analysis are the time-independent Lyapunov exponents $\lambda_{1}, \lambda_{2}, \ldots, \lambda_{N_{\text {lyap }}}$ and the Gram-Schmidt vectors at different time points. The vectors correspond to the columns of $\mathbf{Q}$, each of which in turn consists of all the degrees of freedom of equation 2.99). According to equation 2.62, the Lyapunov exponents are time-averaged growth factors of the perturbations. Due to OsELEDEC's theorem [111, it is known that this average will converge to a well-defined Lyapunov exponent when $t \rightarrow \infty$. However, the time scales on which this happens depend strongly on the attractor the system is evolving on. Heuristically speaking, the system has to sample the attractor densely enough and must have acquired the typical probability of being found in a certain position on the attractor. To recover the Floquet exponents as Lyapunov exponents for a periodic system, either one well-converged trajectory of exactly one period in time has to be averaged, or the averaging time has to be much larger than the periodicity of the system.

In order to obtain a quantitative measure for the accuracy of the obtained Lyapunov exponents, the sum of equation $(2.62)$ is split up to define partial averages of the growth rates as

$$
\lambda_{i}^{(k)}=\frac{K}{m \Delta t} \sum_{l=(k-1) \frac{m}{K}+1}^{k \frac{m}{K}} \ln R_{i i}^{l} \quad \forall k: 1 \leq k \leq K,
$$

where $m$ as in section 2.1 .8 denotes the total number of orthonormalization steps and $i$ is the index of the Lyapunov exponent counting from the largest one. The new parameter $K$ defines the number of partial exponents that are calculated as if the averaging time had been only one $K$ th of its actual value. The reasoning behind this is that the partial exponents will start to deviate from their average (which is just the overall estimated Lyapunov exponent), when the averaging time becomes too short. In contrast, in the limit $m \rightarrow \infty$, i.e. infinite simulation and averaging time, all of the partial exponents 
$\lambda_{i}^{(k)}, k=1, \ldots, K$ converge to the same value, the true Lyapunov exponent $\lambda_{i}$. The standard deviation across the set of partial exponents is taken as an accuracy measure for each calculated Lyapunov exponent $\lambda_{i}$. In order to avoid an accidental match of the averaging time for the partial Lyapunov exponents with a periodicity of the system, the above accuracy estimation is carried out for values of $K$ from 2 to 10 . The maximum of all the standard deviations calculated for different $K$ then serves as an error measure for each Lyapunov exponent. These error measures will be plotted as error bars in section 3.1 when appropriate.

\subsubsection{Hardware, software, parallelization}

All numerical simulations for this thesis were carried out on computer clusters of the Max Planck Institute for Dynamics and Self-Organization in Göttingen. Most of the calculations were done on the 16 machines of the Biomedical Physics Group with 8 cores each (two quad-core Intel ${ }^{\circledR}$ Xeon ${ }^{\circledR}$ X5355 CPUs @2.66GHz) and 32GB of RAM. For some of the simulations, it was possible to use parts of the computer cluster of the Laboratory for Fluid Dynamics, Pattern Formation and Biocomplexity (LFPB), consisting of a total of 72 machines with 12 cores each (two hexa-core Intel ${ }^{\circledR}$ Xeon ${ }^{\circledR}$ X5650 CPUs @2.67GHz) and $24 \mathrm{~GB}$ of RAM.

All computers run a homogeneous Linux environment. The software MediaSim for carrying out the actual calculations is a modular framework for the simulation of extended systems and was completely self-written in $\mathrm{C}++$. Its capabilities exceed those described in this numerical methods section (see appendix C). In the course of the work for this thesis, it has become one of the standard numerical tools in the Biomedical Physics Group and was used and considerably consolidated and extended by several people, first of all D. Hornung [182]. A. Behrend and P. Linke used it for their Diploma thesis and Bachelor's thesis, respectively [183, 184]. Furthermore, A. MAYER extended the framework during the work for his Bachelor's thesis [185], as did H. TOM WÖRDEN [186] in addition to his work as a student research assistant.

Many of the theoretical problems that are addressed in this work using numerical simulations (in particular in sections 3.1 and 3.2 require parameter scans, resulting in a large number of similar numerical simulations. The simplest form of paralellization therefore is to use the hundreds of cores available on the computer cluster to start $N$ of these simulations in parallel. This results in a guaranteed reduction of the computation time to $1 / N$ times the time required for sequential calculation on a single core (apart from marginal losses due to bandwidth limitation during file reading and writing operations). The same kind of trivial parallelization was applied to the analysis of experimental data for section 3.3 , which was implemented in MATLAB ${ }^{\circledR}[187$.

If the number of available cores exceeds a small number of computationally intensive numerical simulations which have to be carried out at the same time, the numerical work can be split between different threads, thus using multiple or all cores on a single machine. For the models used in this thesis, the critical order of magnitude, for which a single-core calculation become too time-consuming, is typically reached for long-term, two-dimensional simulations at high spatial resolutions or three-dimensional simulations. In these cases, the computational domain is subdivided into strips of equal width. Each thread then 
carries out the calculations for all computational points in one strip. This parallelization technique is implemented in the framework MediaSim on the level of array operations and is therefore available to all higher-level algorithms. For the calculation of Lyapunov exponents and vectors, not only the QR-decomposition is parallelized (as described in section 2.2.6), but also the time stepping in tangent space, where different perturbations are propagated in different threads. The threading strategy could in general be extended to computations distributed across different machines, e.g., via message-passing interface libraries, but this has not been necessary so far. 


\subsection{Experimental methods}

A global measure for the electrical activity of the heart 10 can be obtained via an ECG. Intracellular or surface electrodes can provide local information on the membrane potential of single cells or small tissue regions. However, for a detailed investigation of activation patterns in the heart, information with high spatial and temporal resolution on the excitatory state of the tissue is required. This has become possible through the use of so-called voltage-sensitive or potentiometric dyes. These are fluorescent molecules which embed into the cell membrane and whose spectral properties depend on the membrane voltage through field-induced charge shift. They were discovered at the end of the 1960s in studies investigating general optical properties of nerve fibers and their changes due to propagating action potentials [188, 189, 190]. Soon after this proof-of-principle in nerve fibers was accomplished, the cardiac community recognized the chances and opportunities of this new technique [191, 192]. After the discovery and characterization of the potentiometric probe di-4-ANEPPS, it became one of the standard dyes in the cardiac field due to its sub-millisecond response time, high sensitivity, long-term stability and comparably low phototoxicity [193, 194, 195, 11 This dye is used for all measurements in section 3.3 which will be described in the following 12 .

\subsubsection{Setup and tissue preparation}

Most of the recordings presented in section 3.3 are obtained on isolated atrial or ventricular preparations of adult beagle dogs. The experimental procedures for these animals were approved by the Institutional Animal Care and Use Committee of the Center for Animal Resources and Education at Cornell University. The procedure for tissue preparation is described in detail in the methods sections of references [201] and [135]. Briefly, after excising the heart from anesthetized adult beagle dogs, physiological conditions are maintained by perfusing the tissue with $37^{\circ} \mathrm{C}$ tyrode solution through the cannulated coronary arteries. The perfusion is also used to administer all drugs including di-4-ANEPPS. Atria or ventricles were cut open and flattened out in a bath, such that both epicardial and endocardial surfaces could be imaged. The setup for optically imaging cardiac electrical activity (excluding the perfusion) is depicted in figure 2.10. Di-4-ANEPPS in the sample is excited using LEDs (Luxeon LXHL-LM5C Green) with approximately $530 \mathrm{~nm}$ peak wave length. The emitted light is longpass filtered before it is detected by a high-resolution, high-speed camera, either a Photometrics Cascade 128+ or a Vision Research Phantom V7. The former acquires images of a maximum of $128 \times 128$ pixels at a maximum frame rate of about $500 \mathrm{~Hz}$, which can be streamed to the hard disk of a PC in real time. This resolution is sufficient to resolve the spatio-temporal dynamics on the tissue surface during normal or arrhythmic activity and to accurately observe the activity induced by electricfield stimulation of quiescent tissue with low field strengths up to approximately $1.5 \mathrm{~V} / \mathrm{cm}$ (see sections 2.3.3 and 2.3.4 for details). Due to limited buffer size, only short recordings of $600 \times 800$ pixels at $2 \mathrm{kHz}$ were acquired with the Phantom camera for validation purposes

\footnotetext{
${ }^{10}$ See section 1.2

${ }^{11}$ To optimize measurements for certain experimental requirements - e.g., simultaneous imaging of calcium and voltage or recording of optical signals from below the surface of the tissue - there is a search for alternatives, particularly in the long wave length range 196, 197.

${ }^{12}$ For further information on optical imaging in general, see references [198, 199, 200].
} 


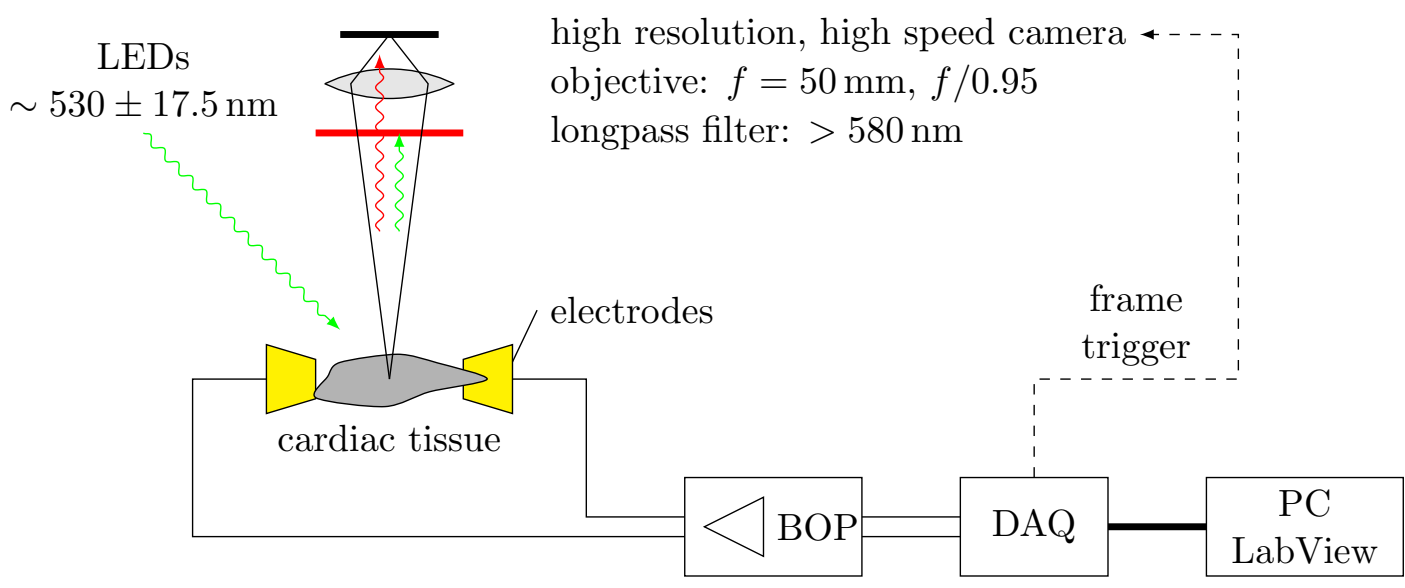

Figure 2.10: Optical mapping setup. LEDs shine excitation light at the sample without additional excitation filters. The perfused cardiac tissue is loaded with voltage-sensitive dye (di-4-ANEPPS), whose excitation and emission spectra depend on the membrane potential. An emission filter is used to convert the specific spectral changes into an intensity change (cf. figure 2.11) that can be recorded with high-speed, high-resolution cameras equipped with EMCCD/CMOS sensors. The voltage between the electrodes is delivered by a power amplifier (Kepco BOP 100-4M), which is controlled by a custom written LabView program through a digital/analog data acquisition device (National Instruments DAQ USB-6259 BNC). Through the same device, the LabView program can trigger the acquisition of individual camera frames.

and to verify that, for electric-field stimulation at higher field strengths when the dynamics is faster, the limited time resolution of the Cascade camera starts to impact the results. Due to nonuniform illumination and staining, the absolute intensity of light detected from different tissue regions varies greatly, which results in motion artifacts, when the tissue is moving. To ensure that there is a nearly constant relation between tissue locations and image pixels in the course of an excitation, Blebbistatin is used to suppress contraction of the tissue [202].

\subsubsection{Optical imaging}

Under these conditions, the intensity change in the recorded images is a measure for the membrane voltage at the corresponding location. The spectral properties of the dye and of the hardware components used in the experiments described here are illustrated in figure $2.11^{13}$ The sample is illuminated using the above-mentioned LEDs without an excitation filter, which is possible due to the relatively narrow-banded light of $35 \mathrm{~nm}$ spectral half-width (green line). The LED spectrum overlaps almost exclusively with the long-wave-length edge of the excitation spectrum of the dye, which shifts a few nanometers to the left when the membrane is depolarized. As the excitation spectrum amplitude in the overlap region is lower during depolarization than at the resting potential, this results in a weaker excitation (hatched area between the dashed curves) of the dye and

\footnotetext{
${ }^{13}$ Optical properties of di-4-ANEPPS were mainly extracted from a recent publication on ratiometric optical mapping [203, which contains collected and combined figures from a number of earlier studies (see also references therein). The properties of the LEDs can be found on the data sheet available at the manufacturer's website http://www.luxeonstar.com/v/vspfiles/downloadables/DS30.pdf (note, however, that the product line has been discontinued).
} 


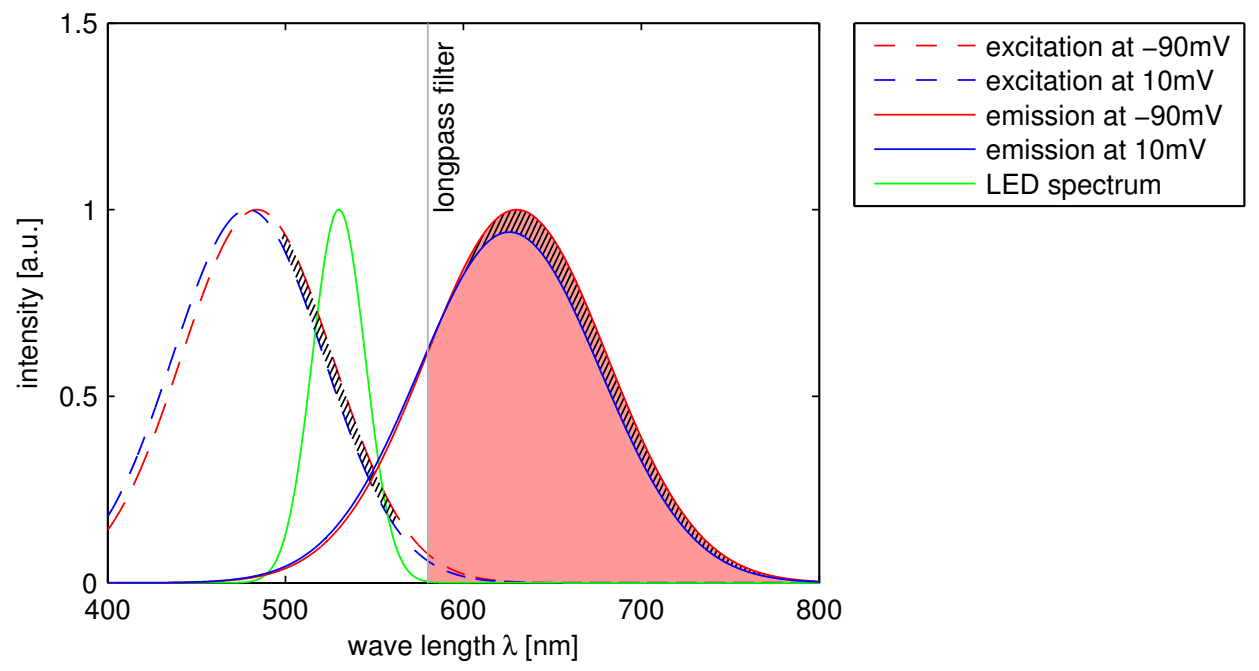

Figure 2.11: Voltage-dependent excitation and emission spectra of di-4ANEPPS. Excitation (dashed lines) and emission spectra (full lines) spectra are plotted for the resting membrane potential (red) and the depolarized state of the cell (blue). The red area under the red curve indicates the total intensity detected for the resting potential through a low pass emission filter. The area between the excitation curves is hatched where the LED intensity (green) is above 10\% of its peak value. The hatched area between the emission curves is the intensity change picked up by the camera due to the spectral shift and amplitude change.

therefore an overall decrease in the emission amplitude, from the (full) red to the blue curve. Additionally, the emission spectrum is also voltage-sensitive and is shifted to the left. Due to the Stokes shift - the separation between the excitation and the emission spectrum - most of the excitation ligh can be kept away from the camera using a $580 \mathrm{~nm}$ longpass filter, such that, at resting potential, the camera detects the integrated intensity indicated by the red area. Due to the combined spectral shift and amplitude decrease, the intensity is reduced during depolarization by the hatched area between the full curves. The optical signal is therefore expected to yield inverted action potentials. The fractional intensity change is typically between $1 \%$ and $5 \%$.

\subsubsection{Electric-field stimulation experiments}

Electric-field pulses are delivered using a power amplifier (Kepco BOP 100-4M) controlled by a custom-made LabView program and two plate electrodes placed on opposing sides of the bath (see figure 2.10). The field strength in the bath is calibrated by measuring the voltage between two wire electrodes placed at a known distance. For all experiments presented here, except those in section 3.3.6, monophasic electric-field pulses with a duration of $T_{\text {pulse }}=5 \mathrm{~ms}$ were used (see figure 2.12). The experiments were carried out by A. Squires, F. H. Fenton, S. Luther and the author of this thesis. The work for this thesis led to significant improvements in the LabView software, automating the data acquisition for electric-field stimulation at different field strengths and with the different pulse shapes shown in figure 2.12. The follow-up experiments presented in section 3.3.6 use this new software and were carried out by M. CheBbok, D. Hornung and the author. 

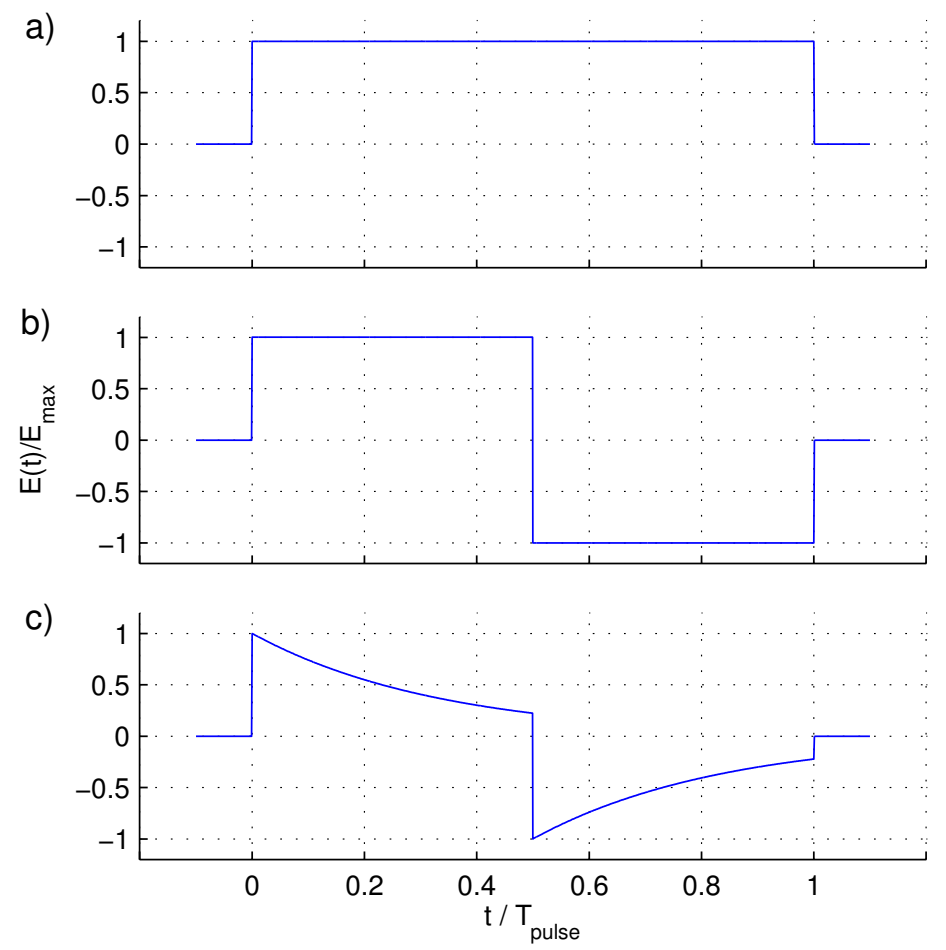

Figure 2.12: Pulse shapes. Different pulse shapes used for stimulating the tissue. All waveforms are relative to the peak amplitude $E_{\max }$ and the total pulse duration $T_{\text {pulse }}$. a) Monophasic pulse b) Biphasic pulse c) Truncated exponential biphasic pulse mimicking a capacitor discharge. This waveform has another parameter $\tau_{\text {decr }}$ denoting the damping time constant. For the first half of the pulse, $E(t)=E_{\max } \exp \left(-t / \tau_{\text {decr }}\right)$. 


\subsubsection{Signal processing - Activation maps}

The raw images are acquired by the camera at a frequency of $0.5 \mathrm{kHz}$ to $2 \mathrm{kHz}$ (depending on camera type and binning parameters) and then processed as depicted in figure 2.13 . The area enclosed by the yellow line in the raw image of figure 2.13 ) was determined retrospectively after carrying out the analysis. It contains a region that lies entirely within the tissue of one chamber (in this case: the right atrium) and has sufficient staining to provide a fractional intensity change (above noise level; approximately $>0.5 \%$ ) which allows for reliable upstroke detection. In figure $2.13 \mathrm{~b}$ ), the averaged signal within the mask shows a single activation of the tissue which was evoked by an electric field pulse of $5 \mathrm{~ms}$ duration and field strength $E=0.22 \mathrm{~V} / \mathrm{cm}$. To reduce noise in the individual time traces for each pixel as plotted in figure 2.13 ), a box filter is applied spatially ( 5 pixels $\times 5$ pixels) and temporally (3 frames). This results in time series for every pixel according to figure $2.13 \mathrm{~d}$ ). Each time series is then normalized by its individual minimum and maximum values to vary between 0 and 1 , leading to the data as in figure 2.13). From these time series, the activation of the tissue can be reliably detected using a simple threshold, usually 0.5. For the examples plotted in figure 2.13), one can see that the tissue is activated earlier at the red location marked in figure 2.13 ) than at the blue one. Assuming that the tissue is activated everywhere only once within a certain time window, an activation map can be constructed from the processed data:

$$
T_{a}(x, y):=\min \{t \mid \hat{I}(x, y, t)<0.5\} \quad \forall x, y \text { within mask }
$$

$\hat{I}$ is the preprocessed and normalized intensity as shown in figure 2.13 ), which depends on the spatial coordinates $x, y$ and time $t$. For each camera pixel, $T_{a}$ displays the time of activation, reducing the activation information contained in the time-varying signals to one static image. To reduce the probability of misdetections due to noise, an activation time is only accepted as such if the average value of the next 10 frames is also subthreshold. One common application of activation maps is the characterization of conduction velocities for arbitrary wave patterns [204, 205, 206, 207]. An example map for the same data as in figure 2.13 is shown in figure 2.14. The earlier activation of the tissue at the location circled in red compared to the region circled in blue can be read off directly from the map (compare figure 2.13e) and f)).

When interpreting activation maps, one has to bear in mind that activity is only observed on the surface of the tissue. Wave fronts on the surface correspond to iso- $T_{a}$ lines, but the conduction velocities estimated from the advancing wave fronts on the surface can at best be considered an apparent velocity, since the angle of inclination with of the wave front with the surface is not known. Likewise, minima in an activation map correspond to points from which waves spread outwards. These are marked by crosses in figure 2.14 a). Such locations can be considered apparent wave sources, but in general correspond to the breakthrough sites, where waves from different real wave source in the bulk of tissue appear first on the surface. Assuming an approximately constant conduction velocity within the tissue, it is reasonable to suspect that each of the apparent wave sources corresponds to at least one real wave source and the activation time of each apparent wave source is correlated with the distance of a real wave source from the surface [208]. Additionally, there could be an intrinsic latency of the onset of a propagating wave for near-threshold 
a)

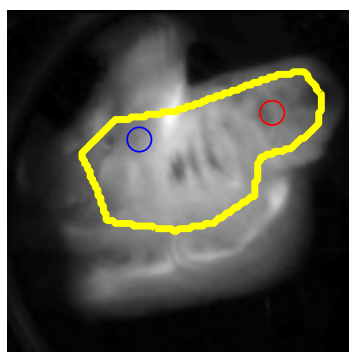

c)

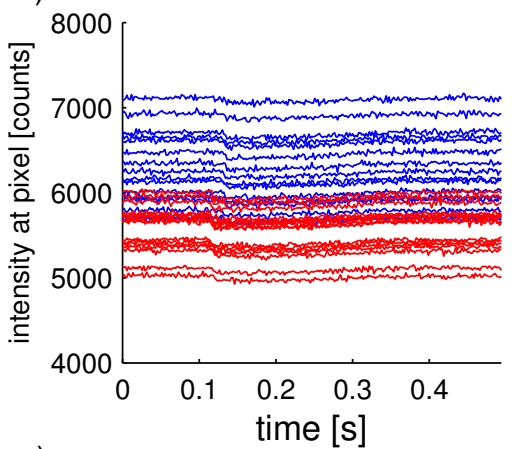

e)

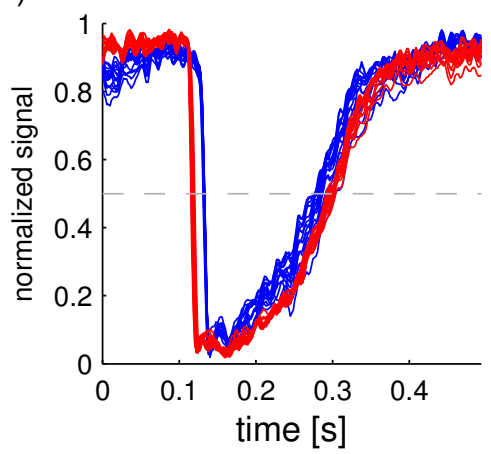

b)

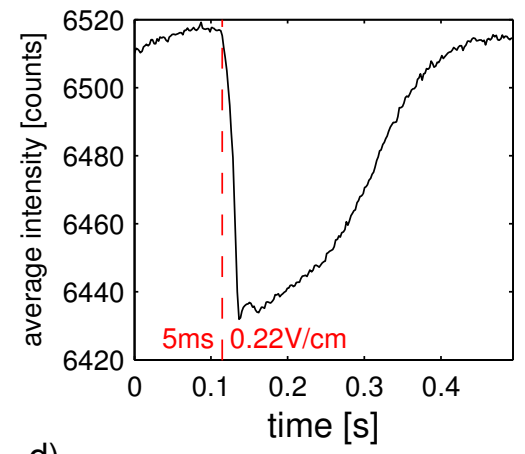

d)

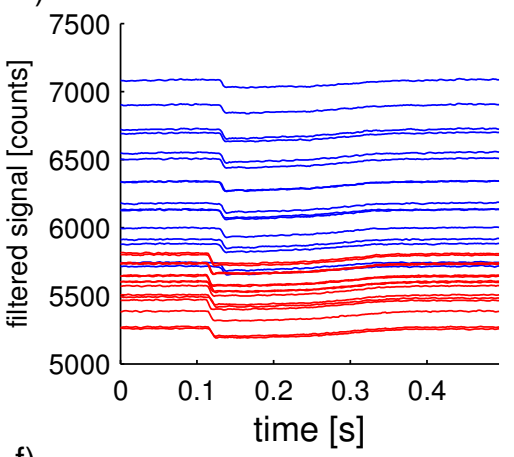

f)

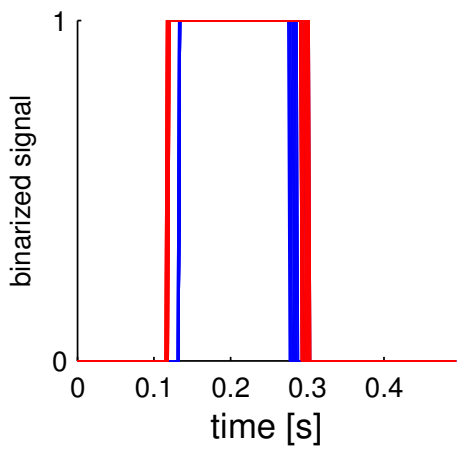

Figure 2.13: Signal processing of optical mapping data. a) Raw camera image showing the endocardial view of the sample. The yellow line indicates the mask of sufficient staining and fractional intensity change above noise level in the right atrium. b) Average signal within the masked region after an electric field pulse of $5 \mathrm{~ms}$ duration at a field strength of $0.22 \mathrm{~V} / \mathrm{cm}$, indicated by red dashed line. c) Raw signals at locations indicated by colored circles in a). d) Spatially and temporally filtered signals at the same locations. e) Signals normalized to maximum and minimum intensity. The dashed gray line indicates the threshold of binarization. f) Binarized signal for activation detection. 
a)

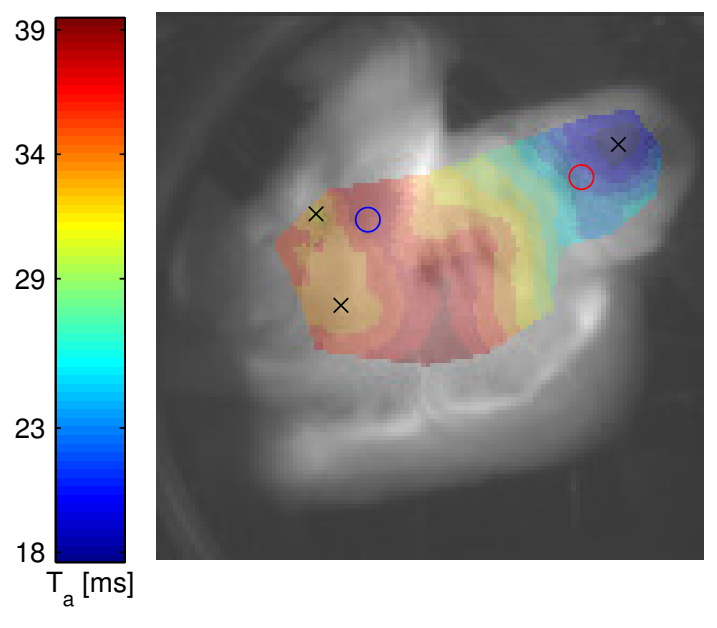

b)

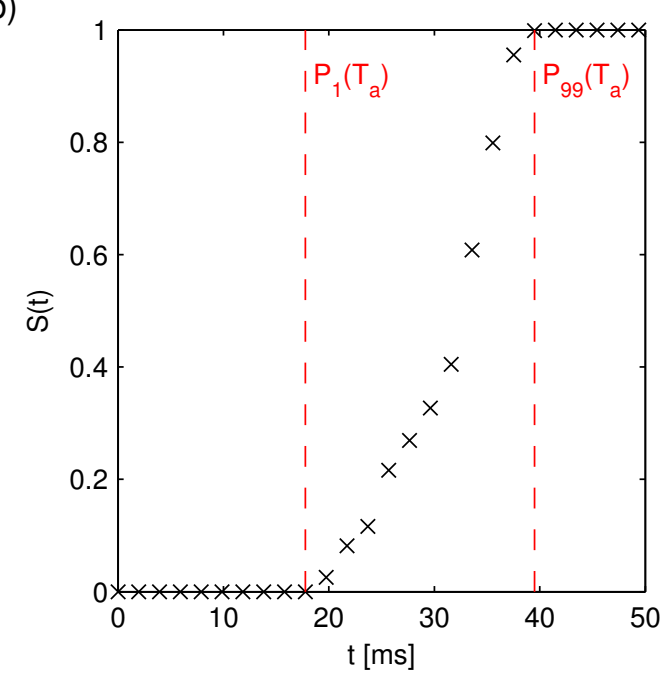

Figure 2.14: Activation map. a) Activation map $T_{a}$ superimposed on a raw image of the atrial tissue preparation. Circles indicate the locations considered in figure 2.13, crosses mark minima.

stimulation. In figure 2.14 ), the two apparent wave sources on the left appear more than $10 \mathrm{~ms}$ later than the one on the right. The activation time $\tau$ is defined as

$$
\tau=P_{99}\left(T_{a}\right)-P_{1}\left(T_{a}\right)
$$

where $P_{n}\left(T_{a}\right)$ is the $n$th percentile of activation times within the masked region. In section 3.3 . $\tau$ will be used to to quantify the number of sources created in the tissue as a response to the electric field. Essentially, this is the time from the observation of the first wave on the surface to the time of full activation of the considered tissue domain. Taking the percentiles instead of the actual minimum and maximum values is a precaution to remove outliers stemming from misdetected activation times. In a graph of the fractional area $S(t)$ activated until a given time $t$ (with respect to the mask), these percentiles can be visualized as depicted in figure $2.14 \mathrm{~b}$ ).

It should be noted that, a priori, the definition of $\tau$ is not equivalent to the time from the stimulation to full activation of the tissue, as the first activation at time $\min _{x, y}\left(T_{a}(x, y)\right)$ can occur some time after the pulse. In order to obtain sufficient statistics, recordings from a large number of experiments carried out over a time of several years (even before the beginning of the work for this thesis) had to be screened in terms of optical signal quality and stability of the preparation. For many of those measurements, camera recordings and electric-field stimulation were initiated independently of each other, rendering a determination of the stimulation time relative to the recorded frames impossible. Instead, the definition of equation 2.103 will be used, which is independent of the actual time of the pulse. There are, however, two reasons why this definition is not expected to alter the results significantly: Firstly, as will be seen in the development of the theoretical tools in section 3.3, the approximations derived there are only valid for a large number of wave sources distributed approximately homogeneously throughout the tissue. If this is the case, then there will certainly be a wave source near the surface of the tissue, calibrating $P_{1}\left(T_{a}\right)$ 
to the time of the pulse. Secondly, the same theory will assume that waves propagate with a constant velocity from the wave sources, starting instantaneously at a given time. However, in reality, there may be a certain latency in the response of the tissue, especially for lower field strengths. Furthermore, the pulse has a finite duration, so the starting time of wave propagation is not well defined even without latency, and using either the beginning or the end of pulse as a reference time is inaccurate, too. From this viewpoint, assuming that the waves begin to propagate when the first activation on the surface is observed might even be a more realistic approximation. For consistency, the activation time $\tau$ as defined in equation (2.103) is used for all results presented in section 3.3 . 


\section{Chapter 3}

\section{Results}

\subsection{Quantification of dynamical complexity in heterogeneous excitable media}

As indicated in the introduction, in this section, the generic view of cardiac tissue as an excitable medium is adopted to investigate, how activation patterns in these systems can be characterized. While the approach chosen here is purely theoretical, in the long run, such characterization might aid the quantitative assessment of the turbulence and complexity of cardiac arrhythmias in an experimental situation and thus contribute to the development of adaptive control strategies which make use of this information. In the past, there has been fruitful interaction between the fields of cardiac electrophysiology and physics, in particular non-linear dynamics, contributing to the understanding of arrhythmias and their development [209]. The investigations in this section continue on this path by applying Lyapunov stability analysis (see section 2.1.8), which has become a standard method in the field of non-linear dynamics, to excitable media and examine, for the first time, the Lyapunov spectra of typical activation patterns arising in these systems. For this purpose, the mathematical framework of reaction-diffusion equations, corresponding to the mono-domain approximation of the cardiac bi-domain equations introduced in section 2.1.3, and the specific models of section 2.1.6 are used. The results presented below target two central questions: First, how can what is known about Lyapunov exponents for low-dimensional dynamical systems be applied to simple, low-dimensional attractors of the extended, dissipative, high-dimensional systems under consideration? Which additional aspects have to be taken into account? Second, how can this basic knowledge help to characterize the dynamics in excitable media in general and in particular in heterogeneous substrates?

Using covariant Lyapunov vectors [114] and the so-called domination of the Oseledec splitting [210, 211] YANG et al. argue that numerical realizations of extended dissipative systems such as the Kuramoto-Sivashinsky and complex Ginzburg-Landau PDE are characterized by a well-defined number of physical, interacting modes, representing the actual dynamics [117]. Additionally, there are passive, isolated, rapidly-decaying modes that originate from the spatial coupling of nearby points. Their number increases with the numerical resolution, whereas the number of physical modes does not, as long as the resolution is sufficiently good. The working hypothesis (necessary for the results to char- 
acterize the original PDE and not just its discretization) is that this is equally true for reaction-diffusion PDEs of excitable media. Since the analysis will be applied to very high-dimensional systems and thus only the upper part of the Lyapunov spectrum will be calculated, the results are expected to converge for high numerical resolutions, with isolated modes being added in the lower part of the spectrum not included in the analysis. Generally, excitable media have many coexisting attractors: using identical model parameters, they usually support a large number of excitation patterns, depending only on initial conditions. These typically include plane waves, single spiral waves and multiple spiral waves (for large enough systems). Furthermore, by varying the model parameters, bifurcations from stable spiral waves to meandering spirals and to spiral breakup can be observed. Since Lyapunov exponents always characterize a specific attractor of a system and not the system itself, the analyses in the next sections will be carried out with respect the above-mentioned typical attractor types in excitable media.

\subsubsection{Plane waves}

Plane waves traveling along the $x$-axis were initiated in a two-dimensional medium of $6.25 \mathrm{~cm} \times 6.25 \mathrm{~cm}$ size, using the smooth Fenton-Karma model with parameter set FK2 (see table A.2 page 177), isotropic diffusion of $D=1 \mathrm{~cm}^{2} / \mathrm{s}$ and periodic boundary conditions. Due to the periodic boundary conditions, the plane wave does not die out at the medium boundary but continuously reenters the domain from the opposite side. For the parameters used here, a stable periodic activation pattern develops, such that $\lambda_{i} \leq 0$ is expected for all $i$, meaning that no perturbation should grow with time. In figure 3.1, a snapshot of the system is plotted along with the perturbations belonging to the leading Lyapunov exponents. As indicated in the figure, there is one neutrally stable direction with $\lambda_{1}=0$ and all other $\lambda_{i}<0, i \geq 2$. This is entirely consistent with the Lyapunov spectrum of a periodic orbit in an autonomous low-dimensional system, where the neutrally stable direction corresponds to a shift along the trajectory, indicating time invariance of the dynamical system. However, in this case, the Lyapunov vectors have a spatial structure: They are concentrated at the wave front and back and show a characteristic vertical oscillation in space. The first vector (second column) indeed corresponds to a shift along the trajectory, accelerating the plane wave towards a future state. A cut along the $y$-axis of the first 15 Lyapunov vectors, shown in figure 3.2 , indicates that the decay rate (cf. figure legend) of the perturbations increases with the spatial frequency. The harmonic spatial shape of the perturbations suggests to attribute their existence to the diffusive spatial coupling of the excitable medium. The emergence of the observed spectrum will be explained in the following.

Indeed, a plane wave in the two-dimensional system can be viewed as the natural extension of a one-dimensional scenario: Because of the absence of spatial variations in $y$-direction, the two-dimensional version of equation 2.72, which describes the actual dynamics, reduces to a one-dimensional problem:

$$
\frac{\partial u}{\partial t}=D(\frac{\partial^{2} u}{\partial x^{2}}+\underbrace{\frac{\partial^{2} u}{\partial y^{2}}}_{=0})+f(u, \mathbf{h})=D \frac{\partial^{2} u}{\partial x^{2}}+f(u, \mathbf{h})
$$




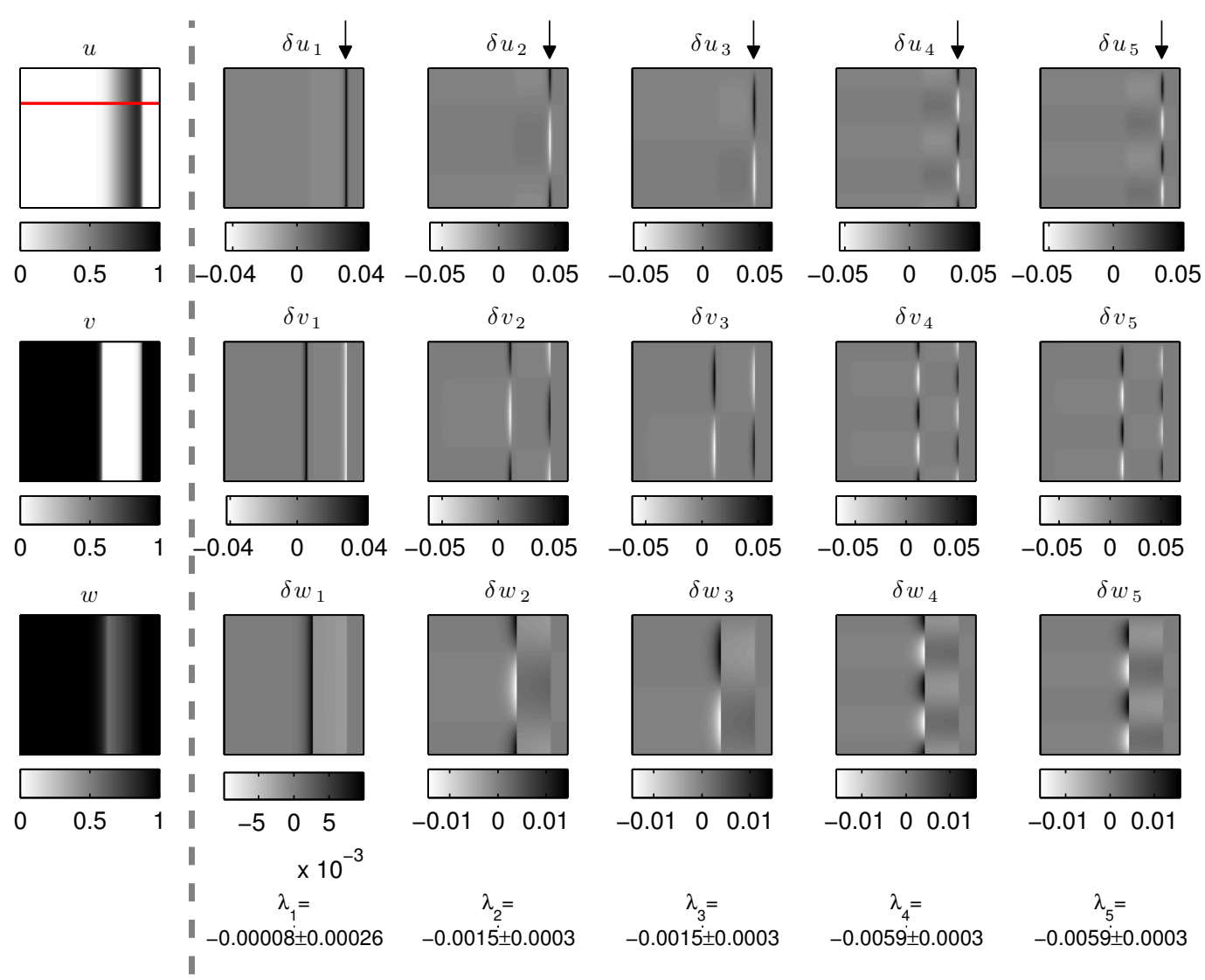

Figure 3.1: Example perturbations of a plane wave. The plane wave is traveling from left to right in a square medium with periodic boundary conditions. In the leftmost column, a snapshot of the medium state at an arbitrary point in time is depicted, the five columns on the right show snapshots of five associated Lyapunov vectors (at the same time), ordered by growth rate. For the first Lyapunov vector $\lambda_{1} \approx 0$, since the plane wave is a periodic orbit in an autonomous system. The higher-index perturbations have negative growth rates, indicated below each column (see figure 3.2 for details). The red line in the upper left plot indicates the domain of a one-dimensional cable which, if considered separately, would exhibit identical dynamics. Simulations done in a $6.25 \mathrm{~cm} \times 6.25 \mathrm{~cm}$ domain with the smooth Fenton-Karma model using parameter set FK2 (see table A.2. page 177), isotropic diffusion of $D=1 \mathrm{~cm}^{2} / \mathrm{s}$ and periodic boundary conditions. The space step is $h=521 \mu \mathrm{m}$, the time step $\Delta t=12.5 \mu \mathrm{s}$. Lyapunov vectors were allowed to align for $2800 \mathrm{~ms}$ before calculating the spectrum according to section 2.2 .6 for another $2800 \mathrm{~ms}$ (roughly 13 periods). 

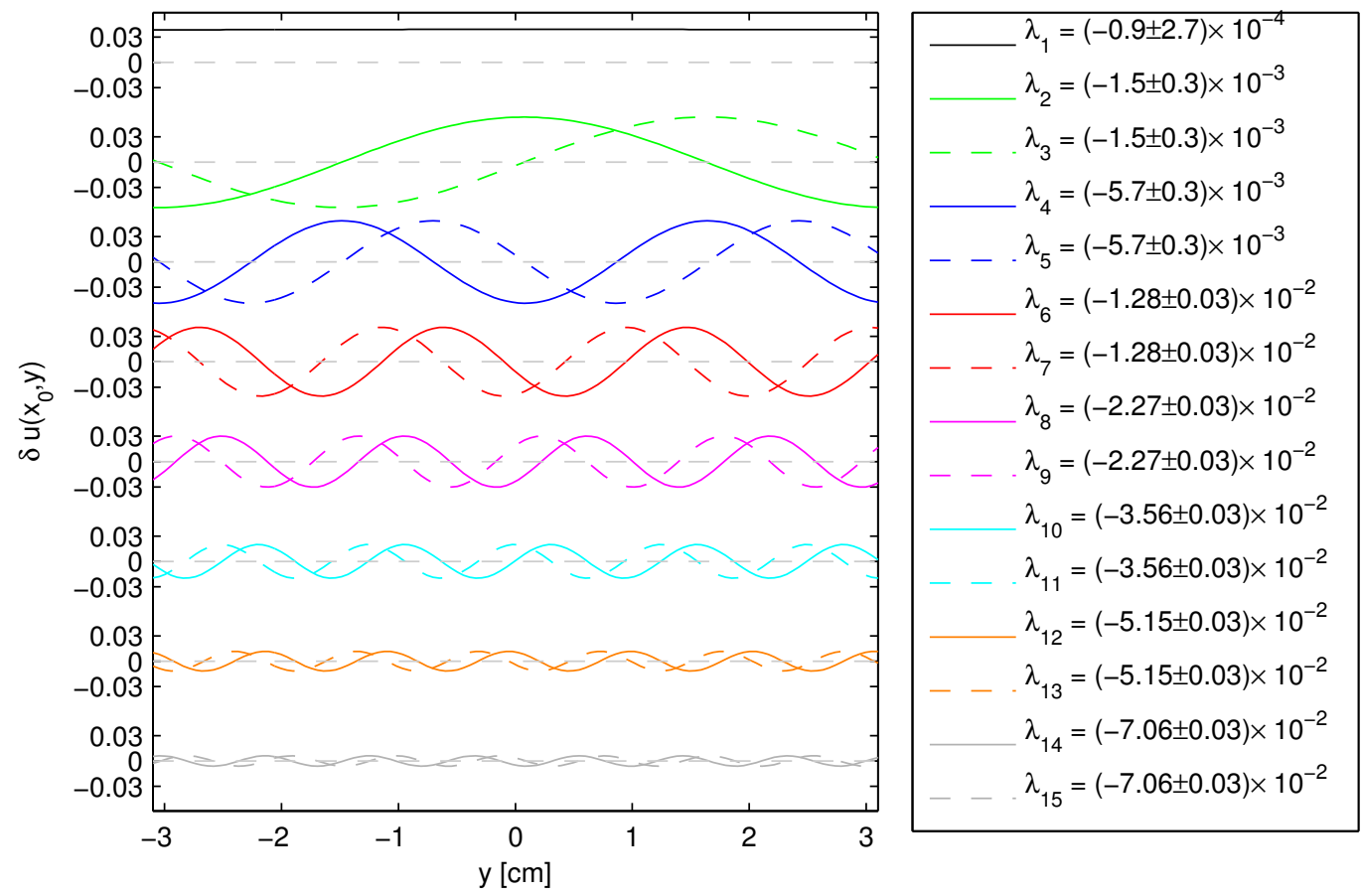

Figure 3.2: Wave front modulation for a plane wave. The plot shows a cut along the wave front (parallel to the $y$-axis) through the $\delta u$-component of the first 15 Lyapunov vectors. The position $x_{0}$ of the cut is indicated by arrows in the first row of figure 3.1 . Model and numerical parameters are identical to figure 3.1 .

This is the equation for a one-dimensional cable (indicated by a red line in figure 3.1). The Lyapunov spectrum of $N$ uncoupled copies of such a cable would consist of $N$ copies of the spectrum of a single cable. However, the diffusion operator $\frac{\partial^{2}}{\partial y^{2}}$ which couples the cables in $y$-direction stabilizes the trajectory where $u(x, y, t)$ is identical for all $y$ and thus shifts some Lyapunov exponents in the negative direction.

Following the above picture, the structure of the Lyapunov spectrum can be explained in the context of transverse Lyapunov exponents for a coupled system of identical oscillators, where one oscillator corresponds to the aforementioned periodic one-dimensional cable. Its $n=3 N_{x}$ degrees of freedom are the values of the three dynamic variables in every of the $N_{x}$ computational points. $N$ of these oscillators (where $N=N_{y}$ is the number of computational points in $y$-direction in the two-dimensional simulation) exhibiting the periodic dynamics of a traveling wave are diffusively coupled in $y$-direction. Assume for a moment that all degrees of freedom of neighboring oscillators are coupled in the same way via diffusion, corresponding to the following system of equations for the Fenton-Karma model (replacing equation 2.40$)$ ):

$$
\begin{aligned}
& \frac{\partial u}{\partial t}=\nabla \cdot \underline{\mathbf{D}} \nabla u-J_{\mathrm{fi}}(u, v)-J_{\mathrm{so}}(u)-J_{\mathrm{si}}(u, w) \\
& \frac{\partial v}{\partial t}=H_{1}(u, v)+\nabla \cdot \underline{\mathbf{D}} \nabla v \\
& \frac{\partial w}{\partial t}=H_{2}(u, w)+\nabla \cdot \underline{\mathbf{D}} \nabla w
\end{aligned}
$$



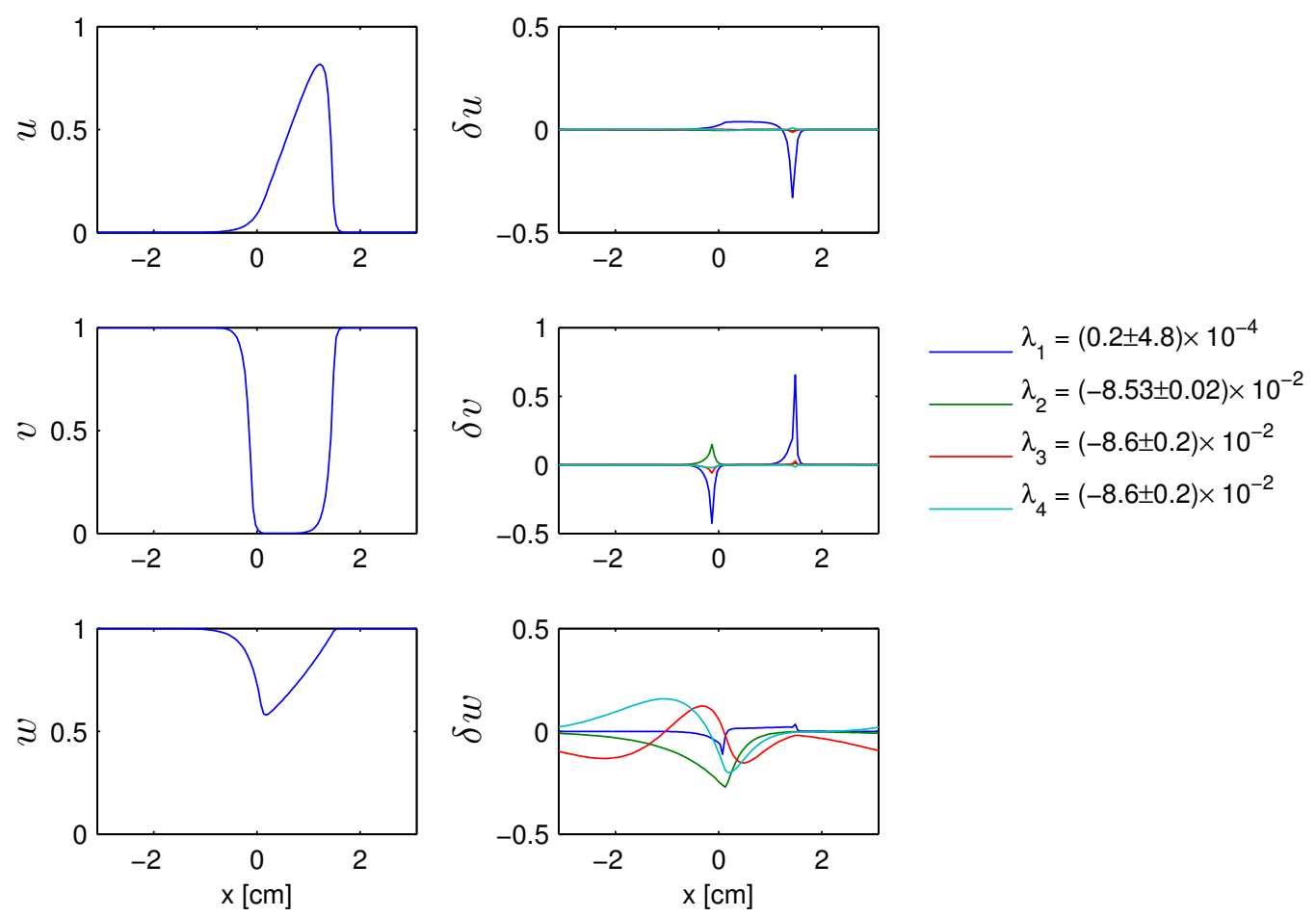

Figure 3.3: Lyapunov spectrum for a traveling wave in a cable. The wave is traveling from left to right in a cable modeled by the modified system 3.2 with periodic boundary conditions. The first column displays the spatial distribution of the model variables $u, v$ and $w$. In the second column, the first four Lyapunov vectors are plotted. Corresponding Lyapunov exponents are indicated in the legend. Numerical parameters are identical to figure 3.1 except for a vanishing spatial extent of the domain in $y$-direction.

$H_{1}(u, v)$ and $H_{2}(u, v)$ denote the original right hand side for $v$ and $w$ of equation 2.40 . A snapshot of the dynamics in one cable together with the leading Lyapunov spectrum is shown in figure 3.3. With this modified system of equations, the transverse Lyapunov exponents of the numerically discretized system viewed as a collection of coupled cables can be calculated analytically, following Heagy, Carroll \& Pecora [212]: They derived an expression for a system of coupled oscillators with an additive coupling term for the $j$ th oscillator of the form $c\left[\mathbf{z}_{j-1}-2 \mathbf{z}_{j}+\mathbf{z}_{j+1}\right]$, where $\mathbf{z}_{j}$ is the state vector of the $j$ th oscillator with its $n$ degrees of freedom. This corresponds exactly to one possible discretization of a second derivative such as the $y$-component of the Laplacian. Equations (2.86) and (2.87) state that the coupling strength in this context is $c=D \frac{1}{h^{2}}=D \frac{N_{y}^{2}}{L_{y}^{2}}$. The result of HEAGY et al. now is that the transverse Lyapunov exponents can be calculated as follows:

$$
\lambda_{i}^{k}=\lambda_{i}^{0}-4 c \sin ^{2}\left(\frac{\pi k}{N_{y}}\right),
$$

$1 \leq k \leq N_{y}-1$ is the index denoting the spatial mode, and $\lambda_{i}^{0}$ with $1 \leq i \leq n$ are the Lyapunov exponents for a single cable without coupling, which were illustrated in figure 3.3. such that $\lambda_{i}^{0}=\lambda_{i}^{1 \mathrm{~d}}$. In the continuum limit $N_{y} \rightarrow \infty$, the origin of this shift 
becomes immediately clear, because for $k \ll N_{y}$

$$
\lambda_{i}^{k} \approx \lambda_{i}^{0}-4 c\left(\frac{\pi k}{N_{y}}\right)^{2}=\lambda_{i}^{0}-D\left(\frac{2 \pi k}{L_{y}}\right)^{2} .
$$

The last term can be recognized as the eigenvalues of the diffusion operator which is responsible for the coupling and whose eigenspaces are two-dimensional:

$$
D \frac{\partial^{2}}{\partial y^{2}}\left(A \sin \left(\gamma_{k} y\right)+B \cos \left(\gamma_{k} y\right)\right)=-D \gamma_{k}^{2}\left(A \sin \left(\gamma_{k} y\right)+B \cos \left(\gamma_{k} y\right)\right) \text {, }
$$

where $\gamma_{k}=\frac{2 \pi k}{L_{y}}$. The fact that the shift of the transverse Lyapunov exponents is given by the eigenvalues of the $y$-component of the Laplace operator is, of course, not a coincidence: Let $\delta \mathbf{U}^{1 \mathrm{~d}}(x)=\left[\delta u^{1 \mathrm{~d}}(x), \delta v^{1 \mathrm{~d}}(x), \delta w^{1 \mathrm{~d}}(x)\right]$ be a Lyapunov vector of the one-dimensional cable associated with a Lyapunov exponent of $\lambda^{1 \mathrm{~d}}$ in the co-moving frame of reference (hence the time independence). Let $\mathbf{L}^{1 \mathrm{~d}}$ denote the linearized dynamics of the cable in the co-moving frame (i.e. the linearization around the fixed point) and $\mathbf{L}=\mathbf{L}^{1 \mathrm{~d}}+\frac{\partial^{2}}{\partial y^{2}}$ that of the two-dimensional system with complete coupling as in equation (3.2) 1 Then, it is known that $\delta \mathbf{U}^{1 \mathrm{~d}}$ is an eigenvector of $\mathbf{L}^{1 \mathrm{~d}}$ with eigenvalue $\lambda^{1 \mathrm{~d}}$. It is seen immediately that with an eigenfunction $f_{k}(y)$ of the Laplace operator with eigenvalue $\gamma_{k}$, the definition

$$
\delta \mathbf{U}(x, y)=\delta \mathbf{U}^{1 \mathrm{~d}}(x) f_{k}(y)
$$

yields an eigenvector $\delta \mathbf{U}(x, y)$ of $\mathbf{L}$ with eigenvalue $\lambda^{1 \mathrm{~d}}-D \gamma_{k}^{2}$. Thus, each choice of $k$ yields a different copy of the spectrum of the one dimensional system in the two-dimensional medium, shifted by $-D \gamma_{k}^{2}$, which is exactly the result of equation 3.4.

Since $\lambda_{1}^{1 \mathrm{~d}}=0$ for a single cable, a number of Lyapunov exponents equal to the second term of equation 3.3 are expected to appear in between $\lambda_{1}^{1 \mathrm{~d}}$ and $\lambda_{2}^{1 \mathrm{~d}}$ (if there is a large enough gap between them). To verify this conjecture, simulations of the full two-dimensional system with the modified equation (3.2) were carried out. As shown in table 3.1, the Lyapunov spectrum of the two-dimensional system corresponds exactly (within numerical errors) to that expected from equation (3.3) for a system of coupled one-dimensional cables: "Copies" of both $\lambda_{1}^{1 \mathrm{~d}}$ and $\lambda_{2}^{1 \mathrm{~d}}$ can be found in the spectrum with their values shifted by the second term of equation (3.3).

In reference [212], HEAGY et al. note that the case of non-complete diffusive coupling is not analytically tractable. This corresponds to the case of the original system equation (2.40), since only the $u$ variables of neighboring cables are coupled. However, even in this situation, the Lyapunov spectra of the plane wave and of the traveling wave in a cable are still closely connected by the spectrum of the Laplace operator: The third column in table 3.2 indicates that all additional Lyapunov exponents between $\lambda_{1}^{1 \mathrm{~d}}$ and $\lambda_{2}^{1 \mathrm{~d}}$ differ from those expected from equation (3.3) only by an approximately constant factor. Also, the two-fold degeneracy of the Lyapunov exponents is preserved. Indeed, even the spatial structure of Lyapunov vectors depicted in figures 3.1 and 3.2 resembles that expected from equation (3.6), namely different oscillatory modulations of the first Lyapunov vector. The similarity of the two scenarios (complete coupling and $u$-coupling) indicates the dominant

\footnotetext{
${ }^{1}$ If diffusion only acts on the variable $u$ as in the original model, $\frac{\partial^{2}}{\partial y^{2}}$ would correspondingly only act on the $\delta u$-component of $\delta \mathbf{U}$, which would lead to a different $\mathbf{L}$ and would invalidate the following argument.
} 
Table 3.1: Lyapunov spectrum of a plane wave for complete coupling. Lyapunov exponents are listed in descending order for plane waves using parameter set FK2 and diffusion in all model variables according to equation (3.2). The first column contains the Lyapunov exponents for a one-dimensional cable (cf. figure 3.3), the second those for the full two-dimensional system. The third column indicates ratio of the observed shift of the Lyapunov exponents to that one expected from equation (3.3). The fourth and firth column denote the values of $i$ and $k$ used for equation (3.3).

\begin{tabular}{ccccc}
\hline$\lambda_{i}^{1 \mathrm{~d}}=\lambda_{i}^{0}$ & $\lambda^{2 \mathrm{~d}}=\lambda_{i}^{k}$ & $\left(\lambda_{i}^{k}-\lambda_{i}^{0}\right) /\left(-4 c \sin ^{2}\left(\frac{\pi k}{N}\right)\right)$ & $\mathrm{i}$ & $\mathrm{k}$ \\
\hline$(0.2 \pm 4.8) \times 10^{-4}$ & $(-0.1 \pm 4.7) \times 10^{-4}$ & & 1 & 0 \\
& $(-9.5 \pm 4.8) \times 10^{-4}$ & $1.0 \pm 0.9$ & 1 & 1 \\
& $(-9.8 \pm 4.8) \times 10^{-4}$ & $1.0 \pm 0.9$ & 1 & 1 \\
& $(-4.0 \pm 0.5) \times 10^{-3}$ & $1.0 \pm 0.2$ & 1 & 2 \\
& $(-9.0 \pm 0.5) \times 10^{-3}$ & $1.0 \pm 0.2$ & 1 & 2 \\
& $(-9.0 \pm 0.5) \times 10^{-3}$ & $1.0 \pm 0.1$ & 1 & 3 \\
& $(-1.60 \pm 0.05) \times 10^{-3}$ & $1.0 \pm 0.1$ & 1 & 3 \\
& $(-1.60 \pm 0.05) \times 10^{-2}$ & $0.99 \pm 0.06$ & 1 & 4 \\
& $(-2.49 \pm 0.05) \times 10^{-2}$ & $0.99 \pm 0.06$ & 1 & 4 \\
& $(-2.49 \pm 0.05) \times 10^{-2}$ & $0.99 \pm 0.04$ & 1 & 5 \\
& $(-3.58 \pm 0.05) \times 10^{-2}$ & $0.99 \pm 0.03$ & 1 & 6 \\
& $(-3.58 \pm 0.05) \times 10^{-2}$ & $0.99 \pm 0.03$ & 1 & 6 \\
& $(-4.86 \pm 0.05) \times 10^{-2}$ & $0.99 \pm 0.02$ & 1 & 7 \\
& $(-4.86 \pm 0.05) \times 10^{-2}$ & $0.99 \pm 0.02$ & 1 & 7 \\
& $(-6.33 \pm 0.05) \times 10^{-2}$ & $0.99 \pm 0.01$ & 1 & 8 \\
& $(-6.33 \pm 0.05) \times 10^{-2}$ & $0.99 \pm 0.01$ & 1 & 8 \\
& $(-7.98 \pm 0.05) \times 10^{-2}$ & $0.99 \pm 0.01$ & 1 & 9 \\
& $(-7.98 \pm 0.05) \times 10^{-2}$ & $0.99 \pm 0.01$ & 1 & 9 \\
$(-8.53 \pm 0.02) \times 10^{-2}$ & $(-8.53 \pm 0.01) \times 10^{-2}$ & & 2 & 0 \\
& $(-8.63 \pm 0.04) \times 10^{-2}$ & $1.0 \pm 0.6$ & 2 & 1 \\
$(-8.6 \pm 0.2) \times 10^{-2}$ & $(-8.63 \pm 0.04) \times 10^{-2}$ & $1.0 \pm 0.5$ & 2 & 1 \\
$(-8.6 \pm 0.2) \times 10^{-2}$ & $(-8.6 \pm 0.2) \times 10^{-2}$ & & 3 & 0 \\
& $(-8.7 \pm 0.1) \times 10^{-2}$ & & 4 & 0
\end{tabular}


Table 3.2: Lyapunov spectrum of a plane wave for single-variable coupling. Lyapunov exponents are listed in descending order for plane waves in the original FentonKarma model of equation (2.40) using parameter set FK2. The first column contains the Lyapunov exponents for a one-dimensional cable (cf. figure 3.3), the second those for the full two-dimensional system. The third column indicates ratio of the observed shift of the Lyapunov exponents to that one expected from equation (3.3). The fourth and firth column denote the values of $i$ and $k$ used for equation (3.3).

\begin{tabular}{ccccc}
\hline$\lambda_{i}^{1 \mathrm{~d}}=\lambda_{i}^{0}$ & $\lambda^{2 \mathrm{~d}}=\lambda_{i}^{k}$ & $\left(\lambda_{i}^{k}-\lambda_{i}^{0}\right) /\left(-4 c \sin ^{2}\left(\frac{\pi k}{N}\right)\right)$ & $\mathrm{i}$ & $\mathrm{k}$ \\
\hline$(-0.8 \pm 2.6) \times 10^{-4}$ & $(-0.8 \pm 2.6) \times 10^{-4}$ & & 1 & 0 \\
& $(-1.5 \pm 0.3) \times 10^{-3}$ & $1.4 \pm 0.5$ & 1 & 1 \\
& $(-1.5 \pm 0.3) \times 10^{-3}$ & $1.4 \pm 0.5$ & 1 & 1 \\
& $(-5.9 \pm 0.3) \times 10^{-3}$ & $1.4 \pm 0.1$ & 1 & 2 \\
& $(-1.32 \pm 0.03) \times 10^{-2}$ & $1.45 \pm 0.06$ & 1 & 2 \\
& $(-1.32 \pm 0.03) \times 10^{-2}$ & $1.45 \pm 0.06$ & 1 & 3 \\
& $(-2.35 \pm 0.03) \times 10^{-2}$ & $1.46 \pm 0.03$ & 1 & 4 \\
& $(-2.35 \pm 0.03) \times 10^{-2}$ & $1.46 \pm 0.03$ & 1 & 4 \\
& $(-3.70 \pm 0.03) \times 10^{-2}$ & $1.47 \pm 0.02$ & 1 & 5 \\
& $(-3.70 \pm 0.03) \times 10^{-2}$ & $1.47 \pm 0.02$ & 1 & 5 \\
& $(-5.37 \pm 0.03) \times 10^{-2}$ & $1.49 \pm 0.01$ & 1 & 6 \\
& $(-5.37 \pm 0.03) \times 10^{-2}$ & $1.49 \pm 0.01$ & 1 & 6 \\
& $(-7.40 \pm 0.03) \times 10^{-2}$ & $1.51 \pm 0.01$ & 1 & 7 \\
$(-8.6 \pm 0.03) \times 10^{-2}$ & $1.51 \pm 0.01$ & 1 & 7 \\
$(-8.6 \pm 0.5) \times 10^{-2}$ & $(-8.6 \pm 0.6) \times 10^{-2}$ & & 2 & 0 \\
$(-8.6 \pm 0.5) \times 10^{-2}$ & $(-8.6 \pm 0.6) \times 10^{-2}$ & & 3 & 0 \\
$(-8.6 \pm 0.5) \times 10^{-2}$ & $(-8.6 \pm 0.6) \times 10^{-2}$ & & 4 & 0 \\
$(-8.6 \pm 0.5) \times 10^{-2}$ & $(-8.6 \pm 0.5) \times 10^{-2}$ & & 5 & 0 \\
$(-8.6 \pm 0.6) \times 10^{-2}$ & $(-8.6 \pm 0.6) \times 10^{-2}$ & & 6 & 0 \\
$(-8.6 \pm 0.5) \times 10^{-2}$ & $(-8.6 \pm 0.5) \times 10^{-2}$ & & 7 & 0 \\
$(-8.6 \pm 0.5) \times 10^{-2}$ & $(-8.6 \pm 0.5) \times 10^{-2}$ & & 8 & 0 \\
$(-8.6 \pm 0.5) \times 10^{-2}$ & $(-8.6 \pm 0.5) \times 10^{-2}$ & & 9 & 0 \\
& & & 10 & 0
\end{tabular}


role of the activation variable $u$ for the formation of wave patterns in excitable media. After all, activation (or, in the Fenton-Karma model: action potentials) can only be initiated from the resting state by a perturbation to $u$, but not via any other variable.

\subsubsection{Rigidly rotating spiral waves}

Another type of attractor that can be found in various examples of excitable media (with no-flux boundary conditions) is that of a single spiral wave. In its simplest form of a rigidly rotating wave, the trajectory of the system corresponds to a periodic orbit. Therefore, at a first glance and from the dynamical systems point of view, the attractor seems to be similar to the one considered in section 3.1.1. However, it is known that spiral waves are characterized by phase singularities (see section 2.1.7), a topological property which represents a localized wave source that does not require specific anatomical features, as opposed to the plane wave that requires the periodic medium considered in section 3.1.1. This qualitative difference between the two attractors becomes manifest in the spectrum of Lyapunov exponents by the appearance of two additional Lyapunov exponents that are equal to zero: In figure 3.4, the subspace belonging to $\lambda_{1}=\lambda_{2}=\lambda_{3}=0$ for a spiral wave in the Barkley model is illustrated with three meaningful directions in tangent space. Due to the degeneracy, Lyapunov stability analysis yields three arbitrary Lyapunov vectors in this subspace. In figure 3.4, three orthonormalized linear combiniations of these arbitrary Lyapunov vectors are depicted, indicating that the two additional zero exponents are a result of the translational symmetry in the system: If the boundary is far away from the rotational center of the spiral - i.e. the two are electrotonically decoupled - a shift of the wave in either of the two available directions will not decay but represents a neutrally stable perturbation that is picked up by the linear stability analysis. The first Lyapunov vector plotted in figure 3.4 (second column) corresponds to a perturbation along the trajectory of the system, i.e. parallel to $\frac{\partial(u, v)}{\partial t}$. This is the neutrally stable direction in tangent space expected for a periodic orbit every autonomous dynamical system, in this case accelerating the dynamics towards a future state. Consequently, the other two linearly-independent directions indeed correspond to a shift of the spiral wave in two different directions. For example, the perturbation in the third column of figure 3.4 accelerates wave parts moving (roughly) to upper left of the medium and decelerates those parts moving to the lower right, effectively shifting the whole spiral towards the upper left.

The periodic orbit of a steadily rotating spiral therefore seems to be embedded in an invariant manifold of total dimension three, where the two directions transverse to the flow represent the independence of the dynamics from position of the spiral tip. Although the finding that spiral waves are subject to a Euclidean symmetry (ignoring boundary effects) seems trivial, note that Lyapunov spectra are computed using a spatially and temporally discretized system. In contrast, the translational, Euclidean invariance of the dynamics is a continuous symmetry. A priori, it is at least questionable whether this continuous symmetry is also present in the discretized system. The analysis shown here confirms that this is indeed the case.

The correctness of the numerical approximation in this particular aspect (with respect to the original PDE) depends on the chosen numerical resolution, as shown in figure 3.5 . For high resolutions, the upper Lyapunov spectrum becomes independent of the numer- 


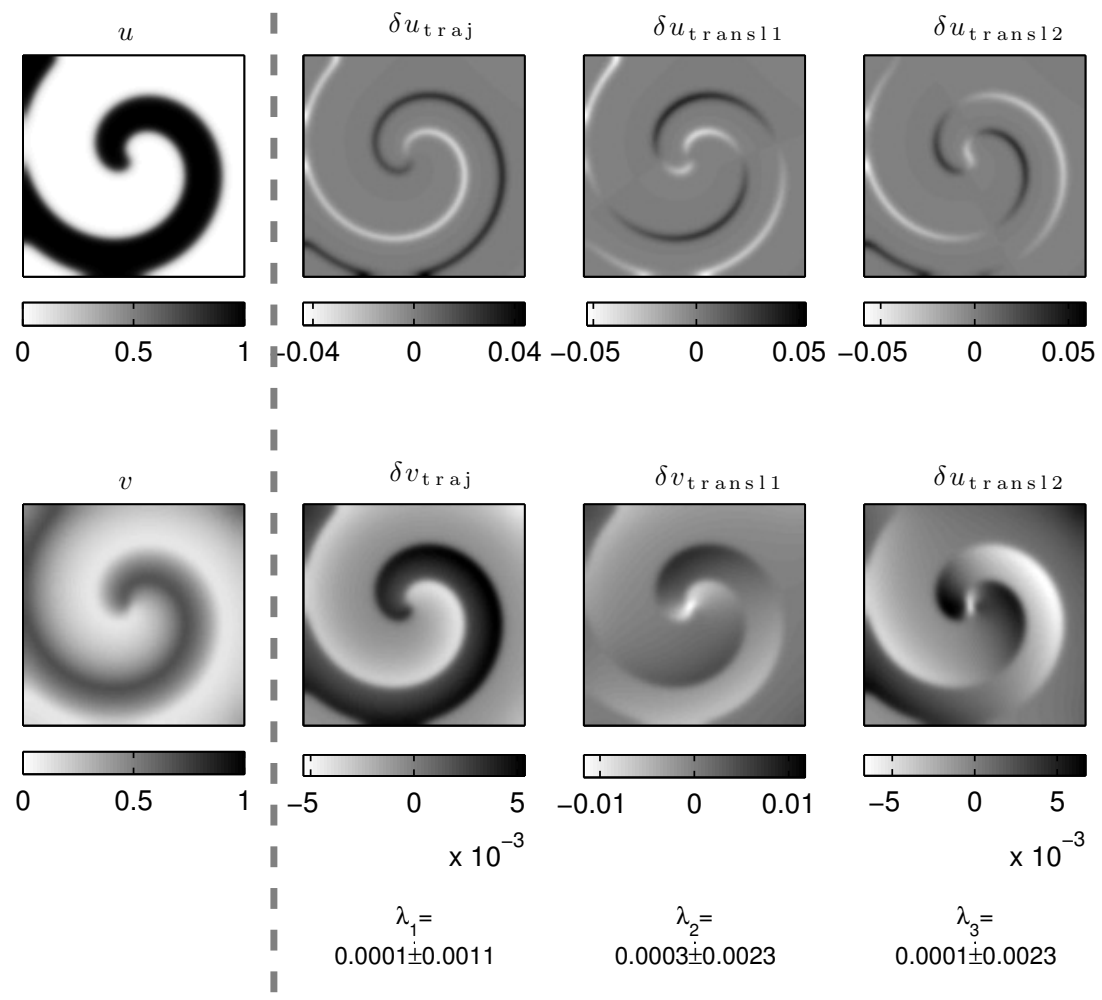

Figure 3.4: Lyapunov vectors of a rigidly rotating spiral wave. Depicted are the medium state (first column) and three Lyapunov vectors (second to fourth column) spanning the degenerated subspace for $\lambda_{1}=\lambda_{2}=\lambda_{3}=0$ (values obtained in the simulation indicated below each column). Linear combinations were chosen such that the second column represents a shift along the trajectory of the system. The third and fourth column correspond to translational Lyapunov vectors that are perpendicular both to each other and to the second column. Simulations done using parameter set B2, see page 177 . The numerical space step is $h=1 / 6$, the domain size $20 \times 20$ and the time step $\Delta t=6.25 \times$ $10^{-4}$. Lyapunov vectors were allowed to align for a time span of 35 time units, before the Lyapunov spectrum was calculated for 350 time units (roughly 100 spiral periods) according to section 2.2 .6 . 


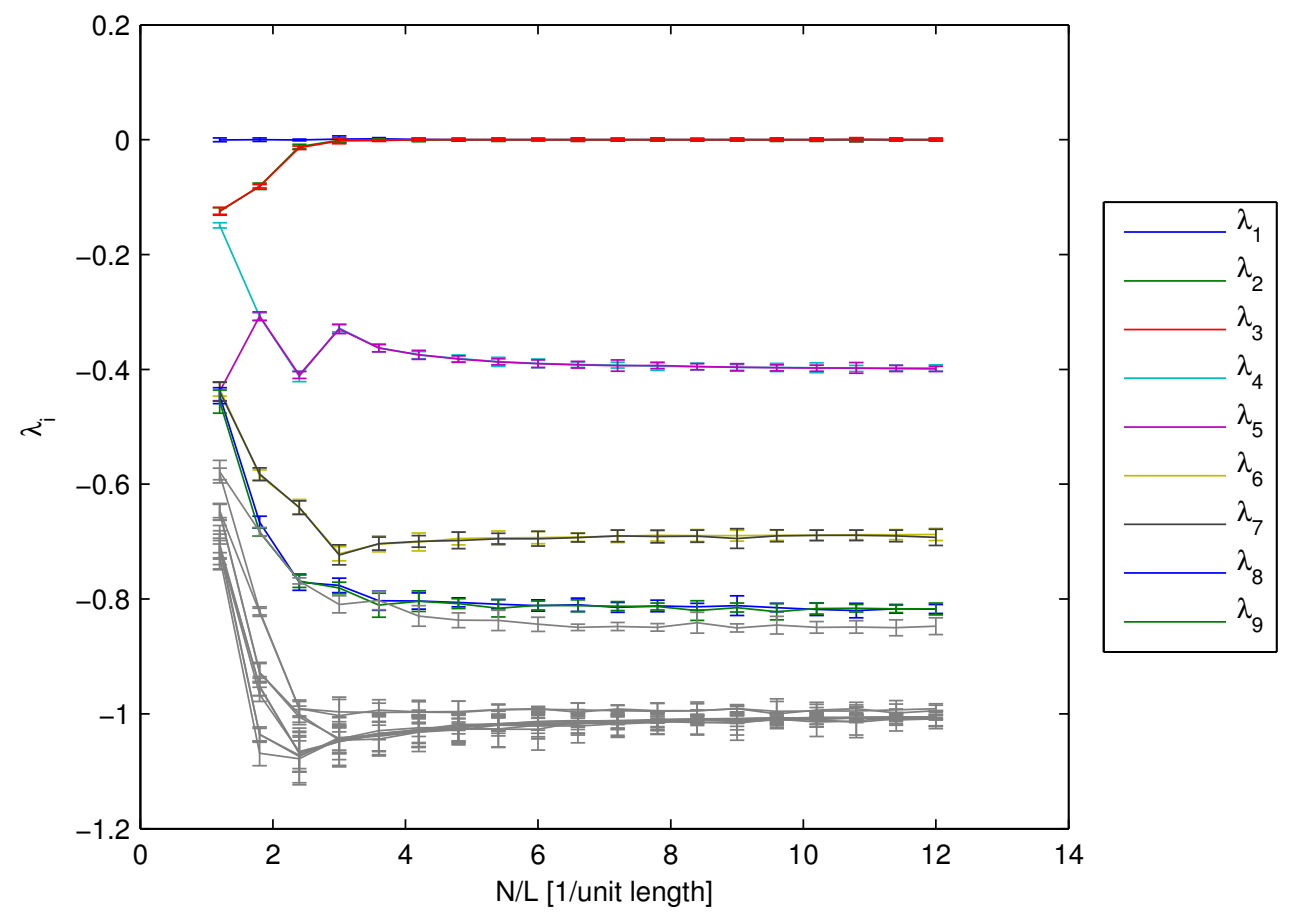

Figure 3.5: Numerical artifacts in the Lyapunov spectrum (Barkley model). Plot of the upper Lyapunov spectrum of a stable spiral wave against the numerical resolution in the Barkley model. As expected, there is a limiting spectrum for large $N / L$ (good numerical resolution). Note the initially stable translational perturbations $\left(\lambda_{2}\right.$ and $\left.\lambda_{3}\right)$ which become marginally stable as the resolution increases. Thus, at sufficiently high resolutions, the translational invariance of the PDE is preserved in the discretized system, whereas pinning of the spiral to the numerical grid is observed at low resolutions. Simulations done using parameter set B2, see page 177 . The numerical space step is indicated in the plot, the domain size is $20 \times 20$, the time step $\Delta t=6.25 \times 10^{-4}$. Lyapunov vectors were allowed to align for 35 time units, before the Lyapunov spectrum was calculated for 350 time units (roughly 100 spiral periods) according to section 2.2.6. 


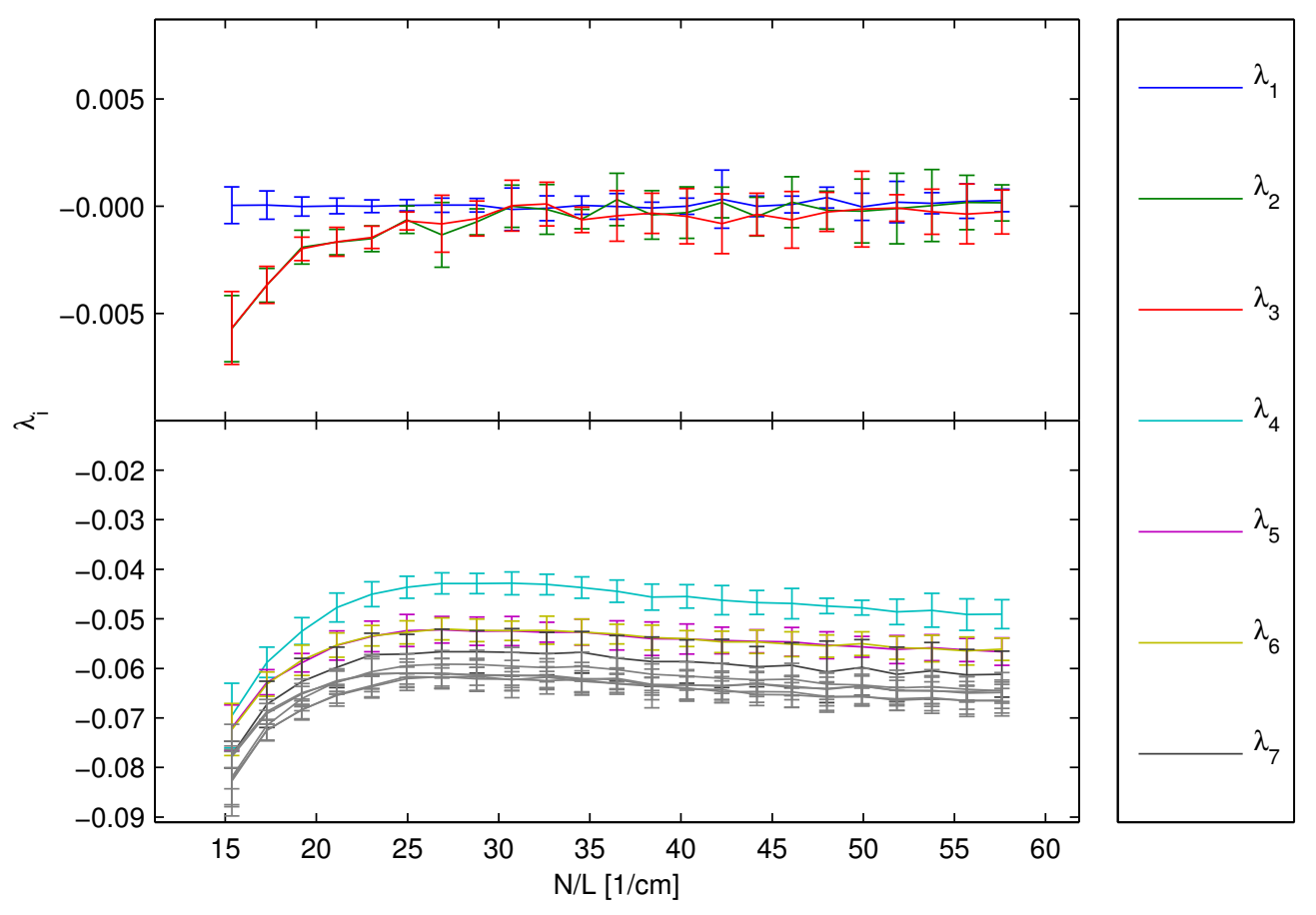

Figure 3.6: Numerical artifacts in the Lyapunov spectrum (Fenton-Karma model). Dependence of the upper Lyapunov spectrum of a stable spiral wave on numerical resolution in the smooth Fenton-Karma model. In contrast to the Barkley model (figure 3.5), the lower part of the spectrum $\left(\lambda_{i}, i>3\right)$ shows increased stability for low resolutions. Note the different y-axis scalings in the lower and upper half of the plot. Simulations done using parameter set FK3 (see page 177) with isotropic diffusion of $1 \mathrm{~cm}^{2} / \mathrm{s}$. The numerical space step is indicated in the plot, the domain size is $3.125 \mathrm{~cm} \times 3.125 \mathrm{~cm}$, the time step $\Delta t=0.0125 \mathrm{~ms}$. Lyapunov vectors were allowed to align for $2800 \mathrm{~ms}$, before the Lyapunov spectrum was calculated for $2800 \mathrm{~ms}$ time units (roughly 22 spiral periods) according to section 2.2.6. 
ical resolution and the structure of the neutrally stable subspace is preserved. For low resolutions, the Euclidean symmetry is broken and the neutrally stable subspace becomes one-dimensional: certain positions of the spiral wave are favored and stabilized by the numerical grid, hence yielding negative Lyapunov exponents for the translational perturbations. In other words, the spiral wave tends to pin to the numerical lattice. This effect has already been noticed by comparing spiral tip trajectories for different numerical resolutions [104]. For stable spiral waves without a visible core (i.e. with a stationary tip), however, this strategy is inappropriate, since a retrospective analysis cannot determine whether or not the tip could potentially have been located at a slightly different position. The plot in figure 3.5 also indicates that a low numerical resolution does not have identical effects on all directions in tangent space: in contrast to $\lambda_{2}$ and $\lambda_{3}$, higher-index Lyapunov exponents increase towards lower resolutions, meaning that the corresponding perturbations become less stable. This effect is, however, not universal: Figure 3.6 indicates that for specific parameters in the Fenton-Karma model, a low numerical resolution has a stabilizing effect on $\lambda_{i}, i>3$ as well. Other authors have observed that a coarse numerical resolution can both induce [104, 213] and prevent [103, 163] spiral wave break-up, which would be associated with one or more Lyapunov exponents being elevated above or reduced below zero, respectively. In particular for investigating control strategies for spiral wave activity in excitable media, Lyapunov stability analysis therefore presents a valuable tool to directly assess the accuracy of the numerical approximation. In this way, the faithful reproduction of stability properties in the numerical realization of the PDE can be ensured without relying on the traditional method of checking the sensitivity of indirect indicators to changes in resolution, such as spiral tip trajectories or periods, conduction velocity, action potential duration and the rate of change of the membrane potential during the upstroke phase [32]. The big advantage of the analysis lies in its implicit check for possible perturbations, whereas all other indicators mentioned above only operate on the attractor itself.

\subsubsection{Multiple spiral waves}

In section 3.1.2, it was shown that Lyapunov stability analysis can detect symmetries in an activation pattern. At a second glance, this is quite surprising for the translational symmetries, since the domain under consideration (a bounded region with no-flux boundary conditions) does not have this symmetry. Consequently, it can be concluded that the part of the activation pattern responsible for the observed zero exponents (and possibly also for other exponents in the upper Lyapunov spectrum) is actually much smaller than the domain size and thus protected from the influence of the boundary ${ }^{2}$. This does not contradict the extension of the corresponding Lyapunov vectors throughout the whole domain (see figure 3.4), as translations and rotations - the latter being equal to a perturbation along the trajectory - nevertheless act globally, as long as the spiral wave can reach the whole domain.

One way to assess the extension of the essential part of the spiral in terms of symmetry and

\footnotetext{
${ }^{2}$ This protection is caused by the waves that are periodically emitted outwards and prevent any interaction in the opposite direction. Indeed, there is a very weak electrotonic coupling even in retrograde direction which decays exponentially with distance from the boundary, thus quickly vanishing from the accessible range of numerical accuracy.
} 
stability would be to investigate the dependence of the spectrum on system size. Here, a different approach is chosen: different numbers of spiral waves are initiated in a medium of constant size, using the Barkley model with parameter set B2 (see table A.1). Figure 3.7a) shows example snapshots of the $u$-variable of the Barkley model for one to twelve spiral waves. The images correspond the state of the medium after an initial transient phase of spiral movement and possible disappearance of phase singularities from the medium. From the plot in figure $3.7 \mathrm{~b}$ ), it can be concluded that the specific signature of three zero Lyapunov exponents for a spiral wave must be carried by a relatively small region around the phase singularity. For one to twelve spiral waves, the number of zero Lyapunov exponents corresponds exactly to three times the number of spirals in the domain. This means that all the symmetries are preserved for each spiral individually. More specifically, this rules out the possibility that any of the spiral waves are phase-synchronized or that the position of one phase singularity is constrained by interaction with the surrounding ones (in which case some zero Lyapunov exponents should become negative). As indicated in figure 3.7 ), this additive behavior of the Lyapunov spectrum does in fact extend to the next (more negative) two pairs of Lyapunov exponents. For the number of spirals and parameters considered here, the entire leading Lyapunov spectrum for $n$ spirals is therefore identical to that one expected for $n$ copies of a system with one spiral wave. This is remarkable, since different $n$ actually represent coexisting attractors in the same system. On the one hand, the behavior of the Lyapunov spectrum reveals that these attractors have a very specific structure. On the other hand, it confirms the intuitive interpretation of spiral waves in excitable media as objects.

To emphasize that the directions in the more and more degenerated subspaces in tangent space can indeed be assigned to individual spiral waves, figure 3.8 shows four specific Lyapunov vectors from the subspace with growth rate zero in a three-spiral simulation. The first one corresponds to the perturbation along the trajectory, while the other three indicate that the time- or phase-shifting symmetry is indeed preserved for each spiral wave individually, as if they were (almost) independent systems. As an interesting by-product, these specific vectors yield a decomposition of the medium into domains controlled by the individual spirals. At least for this periodic attractor, Lyapunov stability analysis therefore provides a way to objectively define "domains of influence" of individual spirals in contrast to visual inspection of the dynamics [105].

It should be noted that the complete preservation of symmetries does not imply that phase singularities in the medium do not interact: as mentioned above, the simulations were allowed to converge to a state with stationary phase singularities exhibiting periodic dynamics. In this transient time, spiral waves can have interacted with each other or pushed each other out of the medium. All the above analysis means is that the phase singularities have settled down outside their mutual interaction ranges, probably because for this specific model and parameters B1, the spiral waves are repelling each other. In contrast, the formation of pairs or clusters of spiral waves that settle down in a minimum of an attracting potential could have been detected, provided the force survives the linearization of the original equations. 
a)

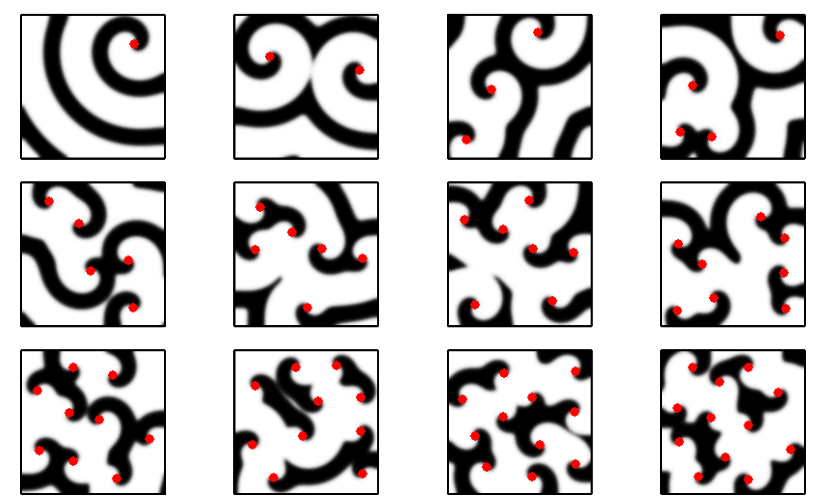

b)

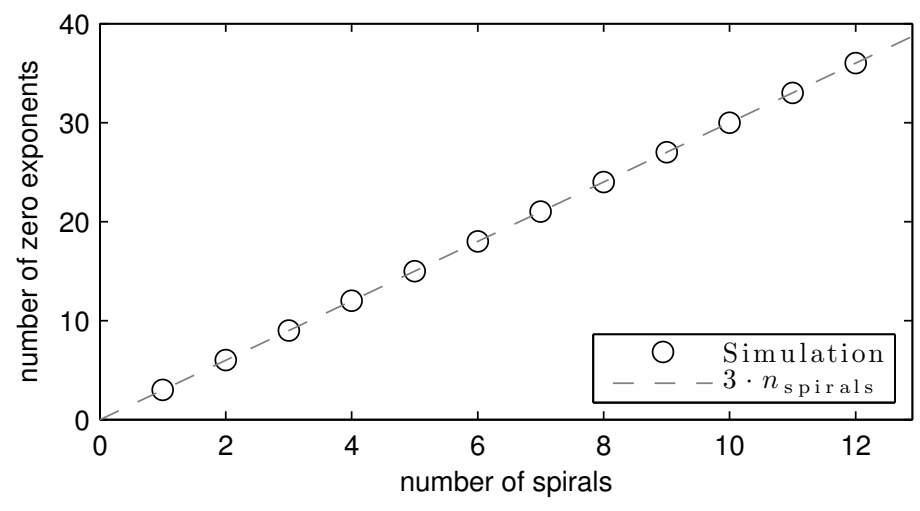

c)

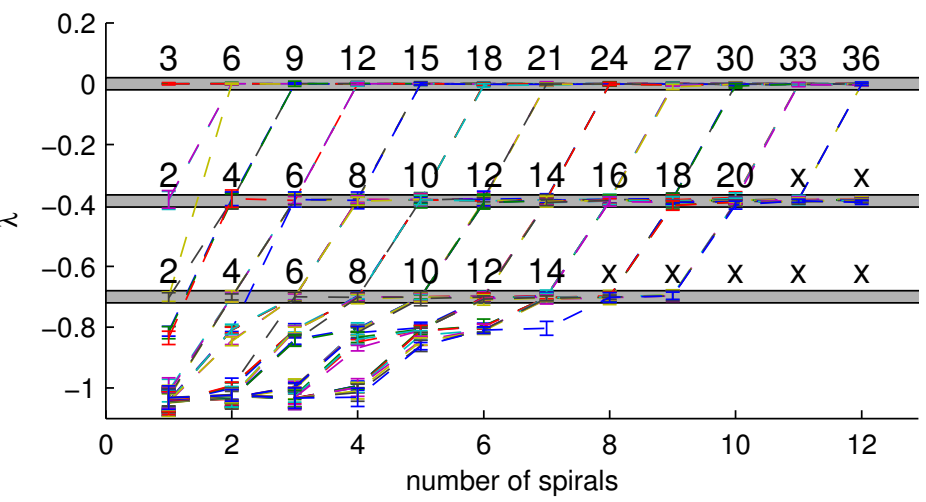

Figure 3.7: Lyapunov exponents of multiple stable spirals. The number of spirals can be detected from the Lyapunov spectrum: a) Exemplary snapshots of the activator variable $u$ of the Barkley model from simulations with one to twelve spirals exhibiting periodic dynamics. Black color indicates activation. Positions of phase singularities are marked by red dots. b) Number of zero Lyapunov exponents for the above simulations. Every spiral contributes three such exponents, indicating the waves' mutual independence in terms of angle and position (all symmetries are preserved individually). c) Lyapunov spectrum of 50 leading exponents plotted against the number of spirals. Dashed lines indicate change of Lyapunov exponents for each additional spiral, Lyapunov exponents are colored randomly by their index. Numbers above gray bands indicate, how many Lyapunov exponents have a value within the corresponding interval of growth rates. Counts that are affected by the limited number of Lyapunov exponents (50) have been replaced by " $\mathrm{x}$ ". Simulation done using parameters B2, see page 177 . The numerical space step is $h=1 / 5$, the domain size $30 \times 30$ and the time step $\Delta t=0.002$. Lyapunov vectors were allowed to align for a time span of 50 time units, before the Lyapunov spectrum was calculated for another 350 time units (roughly 100 spiral periods) according to section 2.2.6. 

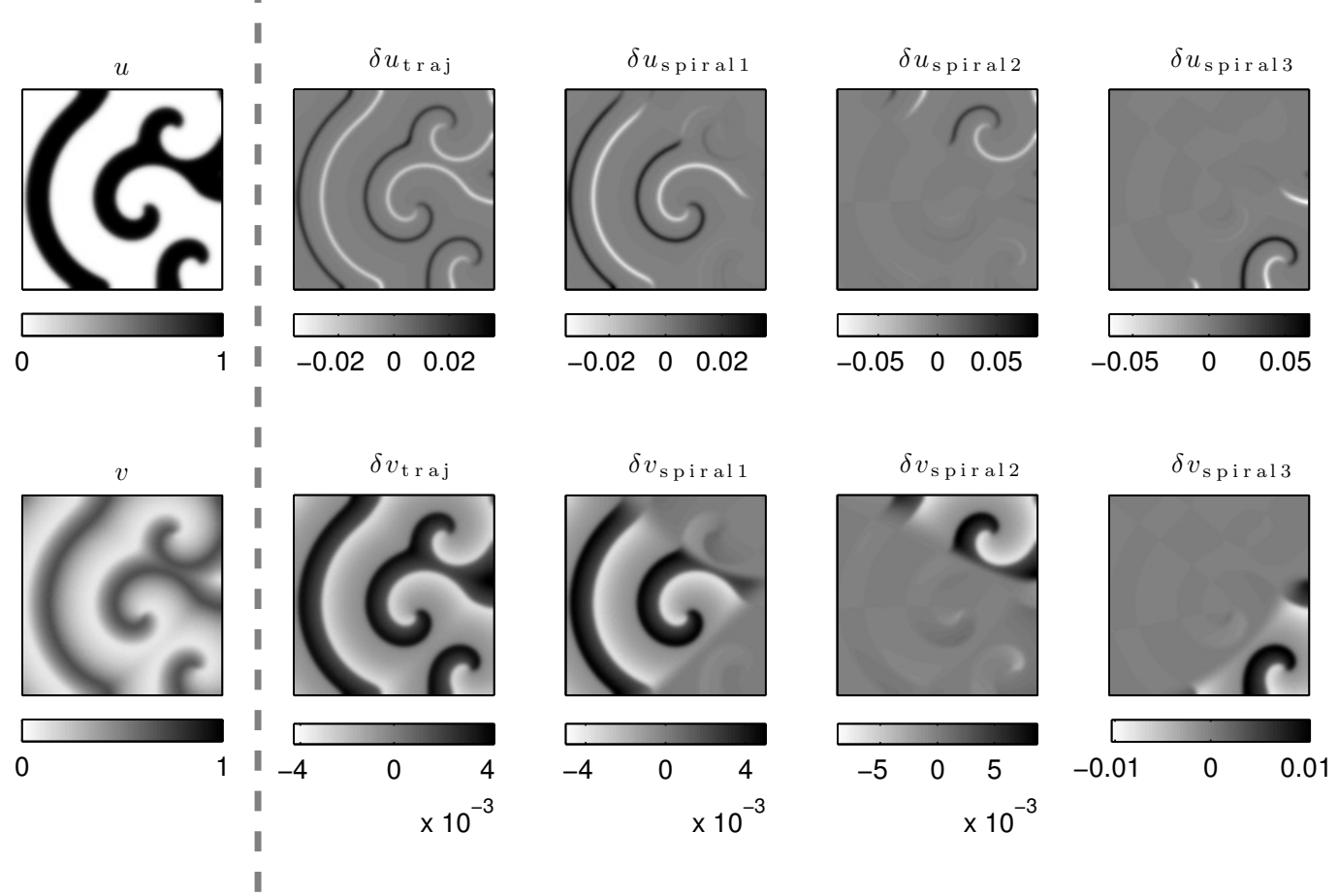

Figure 3.8: Single-spiral perturbations in a system of multiple spirals. Multiple spiral waves on a periodic orbit as independent entities. Along with the state of a medium containing three periodically rotating waves (first column), four specific directions in the degenerate subspace of neutral perturbations are shown. $\delta(u, v)_{\text {traj }}$ is the perturbation along the trajectory of the complete system. $\delta(u, v)_{\text {spiral }\{1,2,3\}}$ are perturbations advancing each spiral individually in time, thereby separating the areas controlled by different spiral waves. Numerical and model parameters are identical to those used in figure 3.7 . 


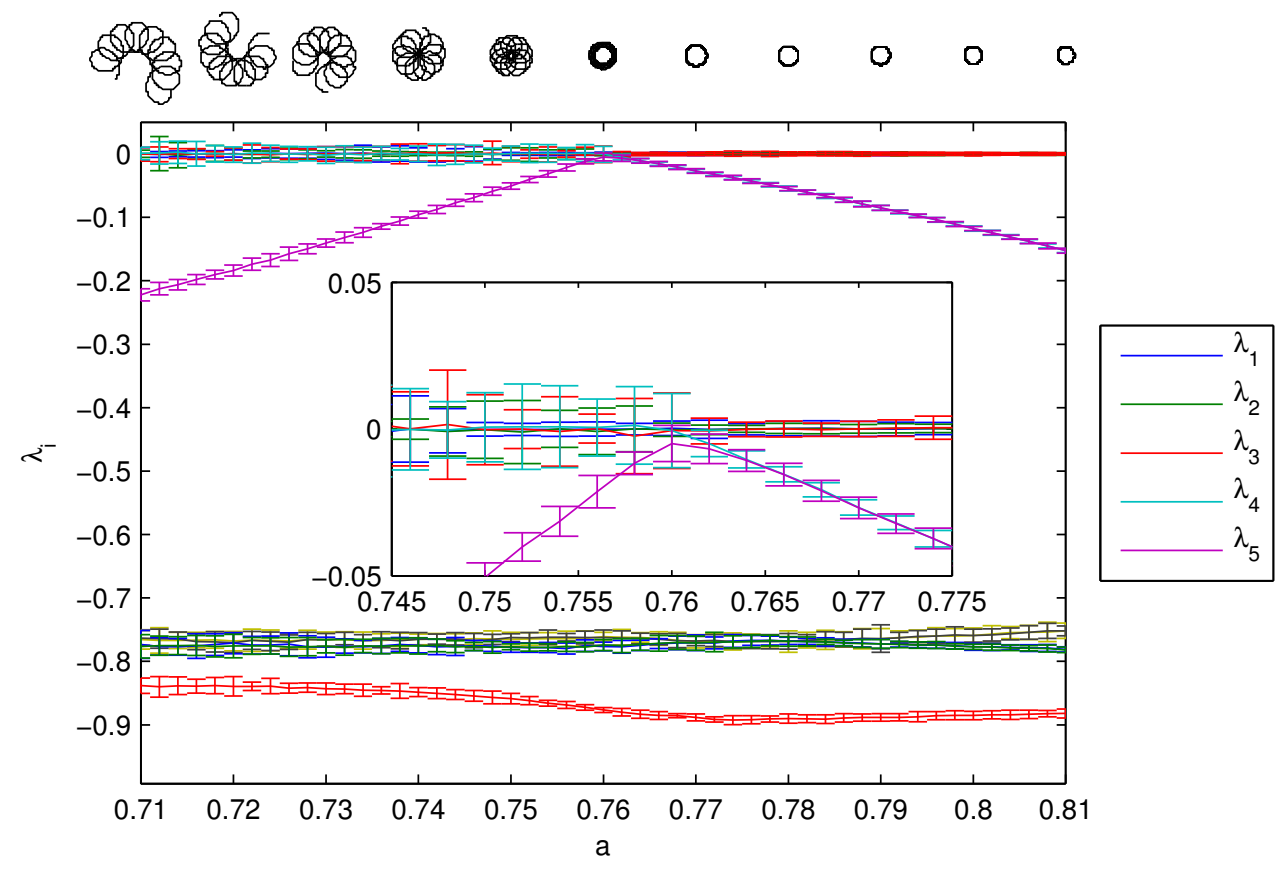

Figure 3.9: Bifurcation detection. Transition from a rigidly rotating spiral $(a \gtrsim 0.760)$ to a meandering spiral $(a \lesssim 0.760)$ for parameter set B3, see page 177 . Spiral tip paths detected by the method outlined in section 2.2.5 with $\left(u^{*}, v^{*}\right)=(0.5,0.4)$ are shown above the plot centered at the corresponding values of $a$. The bifurcation point can be clearly detected from the spectrum of Lyapunov exponents. For meandering, the degenerate subspace of growth rate zero becomes four- instead of three-dimensional, as expected from the Hopf nature of the bifurcation. Inset: Plot enlarged around the bifurcation point. The numerical space step is $h=1 / 8$, the domain size $18.75 \times 18.75$ and the time step $\Delta t=0.002$. Lyapunov vectors were allowed to align for a time span of 80 time units, before the Lyapunov spectrum was calculated for 700 time units (roughly 200 spiral periods independent of $a$ ) according to section 2.2.6.

\subsubsection{Transition to meandering}

One classical use case for Lyapunov exponents is bifurcation analysis: Bifurcations are qualitative changes of the attractor structure along a certain path in parameter space. Locally in phase space, certain directions change their stability properties from stable to unstable or vice versa. Therefore, exactly at the bifurcation, there are typically one or more additional neutrally stable directions that can be detected via Lyapunov stability analysis (and yield additional zero Lyapunov exponents). A typical and well-understood bifurcation in excitable media is the transition from a rigidly rotating spiral to a meandering spiral, which has been shown to occur via a Hopf bifurcation [72, 107, 106] introducing a second frequency into the system. D. BARKLEY obtained this important finding by carrying out a linear stability analysis on the fixed point representing a rigidly rotating spiral in the co-rotating frame of constant angular velocity. In this frame of reference, two eigenvalues of the Jacobian (at the fixed point) cross the imaginary axis at the critical bifurcation parameter value.

Figure 3.9 shows the Lyapunov spectrum for a single spiral wave in the Barkley model using parameter set B3. The transition to meandering occurs from right to left for a parameter value of $a \approx 0.760$ and is identical to the one considered in a publication by BARKLEY 


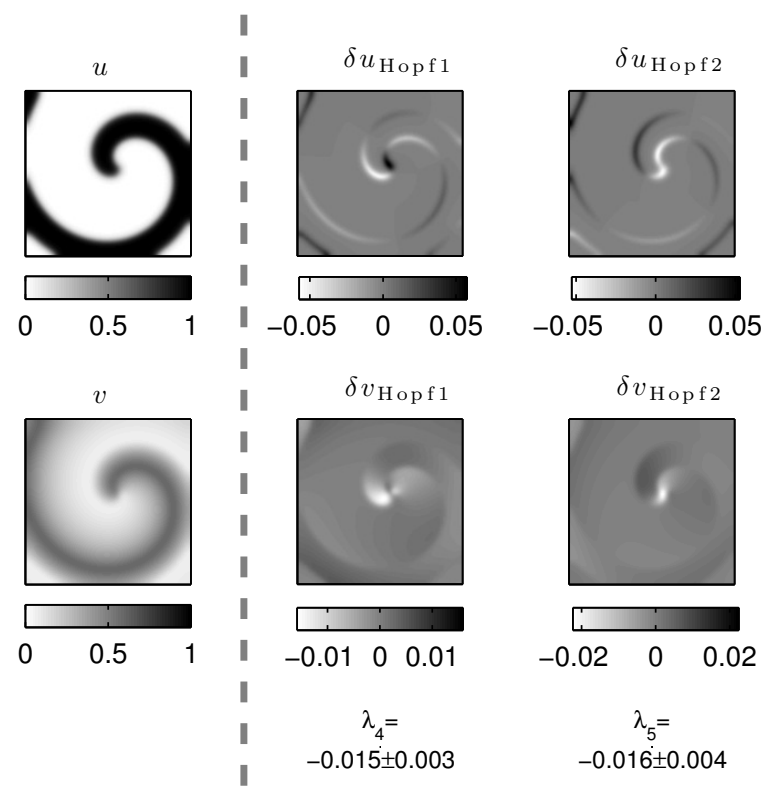

Figure 3.10: Meandering modes for a rigidly rotating spiral. Bifurcating subspace in tangent space for parameter set B3 with $a=0.766$, i.e before the meandering bifurcation (c.f. figure 3.9). Shown is the medium state (leftmost column) and are two arbitrary perpendicular vectors in the degenerated subspace belonging to the fourth and fifth Lyapunov exponent (values indicated below each column).

[107]. Towards the bifurcation point (from the right), two Lyapunov exponents approach zero, corresponding to the two-dimensional eigenspace for the fixed-point problem considered by BARKLEY. At the bifurcation, the rigid rotation of the spiral becomes unstable via the above-mentioned Hopf bifurcation. Since Lyapunov spectra only characterize the attractor the system is currently exploring, the two Lyapunov exponents do not become positive (as the real parts of the eigenvalues for the fixed-point analysis do). Instead, after the bifurcation (towards the left), there are now four zero Lyapunov exponents: two for the periodic or quasi-periodic dynamics on a torus and two - as before - as a result of the Euclidean symmetry (see sections 3.1.2 and 3.1.3). The Lyapunov vectors belonging to the two exponents approaching zero towards the critical parameter value correspond to the bifurcating directions in tangent space. Thus, the two vectors shown in figure 3.10 span the two-dimensional bifurcating subspace. As the Lyapunov vectors depicted in figure 3.4 , which represent the Euclidian symmetry, the Lyapunov vectors for the meandering modes are concentrated at the wave front and back. However, while the symmetry modes have an angular periodicity of the wave front modulation of approximately $2 \pi$ (because of its action as a spatial shift), the meandering modes exhibit a higher spatial frequency. Note that this is similar to the increasing spatial frequency for higher-index modes for plane waves as depicted in figure 3.1

Of course, Lyapunov stability analysis cannot replace linear stability analysis of the corresponding fixed point problem: the most important disadvantage is that the imaginary parts of the eigenvalues cannot be reconstructed from the Lyapunov exponents. Furthermore, as mentioned above, Lyapunov analysis is always restricted to the attractor the system is currently evolving on, while a linear stability analysis can determine the stability properties of arbitrary points in phase space, no matter whether they are stable or 
not 3 The latter might, however, also be considered an advantage, since the fixed points (or other manifolds) do not have to be determined separately from the stability analysis. As an example, the angular wave speed of the co-rotating frame along with the fields of $u, v$ and $w$ of the Fenton-Karma model are obtained using a Newton-Raphson method in reference [108, and the dominant eigenvalues and eigenmodes at the fixed point have to be determined by further sophisticated methods. In contrast, Lyapunov exponents and vectors can be determined by standard procedures without any further algorithmic effort. Note also that the ability to detect and characterize this particular meandering bifurcation via linear stability analysis is based on the geometrically simple trajectory from which it originates, which makes it easiliy possible to transform a periodic orbit into a fixed point. While this can in the general case always be done by defining appropriate Poincaré sections, switching from the continuous to a time-discrete system might hide important details. In contrast, Lyapunov analysis could also be applied for meandering transitions where the (non-meandering) spiral trajectory is not just a circular motion.

\subsubsection{Circular heterogeneities}

So far, the stability analysis has been applied to homogeneous excitable media, only. However, an important problem in the context of the control and development of undesired activation patterns in excitable media is how heterogeneity influences their stability (see introduction, section 1.6). One more specific question one might ask is: If a spiral wave is prone to some sort of instability, does heterogeneity stabilize or destabilize it?

The scenario in which this question will be answered uses a specific parameter range in the Barkley model, in which $a$ and $\epsilon$ are fixed, whereas the excitation threshold $b$ is varied. Figure 3.11 shows the Lyapunov spectra for a spiral wave in a homogeneous medium with the different values of $b$. For the whole range, the spiral wave rotates steadily, indicated by three Lyapunov exponents which are equal to zero (similar to the spiral in section 3.1.4 before the meandering bifurcation, see figure 3.9). The two Lyapunov exponents associated with meandering come very close to zero towards $b \approx 0.13$, but instead of bifurcating, they stabilize again for further increasing $b$. Finally, for very high $b$, the medium loses its ability to form spiral waves and so the Lyapunov spectrum becomes all negative, indicating a stable fixed point which corresponds to the stable, homogeneous resting state of the medium.

The heterogeneity that will be considered is a circular region of radius $R$ close to the spiral tip, in which the excitation threshold is increased from $b=0.08$ (which is used in the rest of the medium) to $b_{\text {het }}$. It is well established that spiral waves are usually drawn to regions of longer spiral wave periods or lower excitability [214, 83, 215], although exceptions are known [82]. For the parameters used here, the expected behavior is indeed observed and the spiral wave is attracted by the heterogeneity if initiated close to it. A snapshot of the long time behavior for an exemplary simulation is shown in figure 3.12 a). From section 3.1 .2 it is known that usually, two Lyapunov exponents which are equal to zero indicate the translational symmetry of the activation pattern. Consequently, for a heterogeneity which pins the spiral wave to a specific position as in figure 3.12 ), these

\footnotetext{
${ }^{3}$ Linear stability analysis can therefore be used to determine the modes that are responsible for instabilities, e.g., meandering and alternans modes for a rigidly rotating spiral in the unstable regime [107, 108.
} 


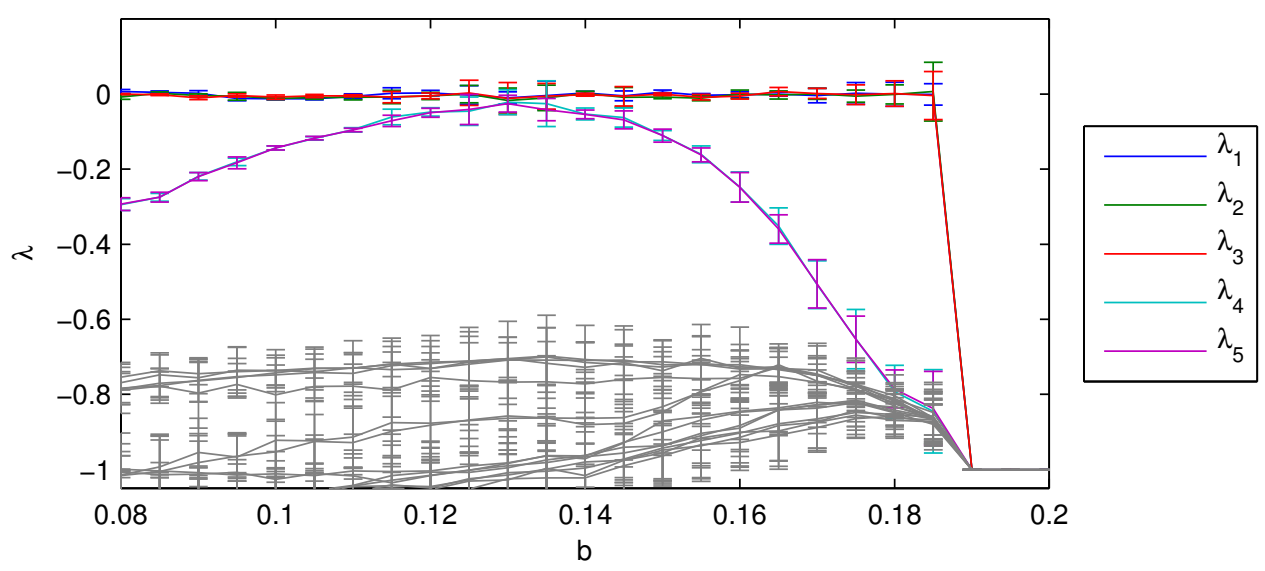

Figure 3.11: Lyapunov spectrum for different homogeneous excitation thresholds. Parameter set B4 (see table A.1, page 177) is used with the indicated value of $b$ throughout the medium. The range of the excitation threshold $b$ covers all values used in the heterogeneous scenario (cf. figure 3.12). The numerical space step is $h=1 / 3$, the domain size $40 \times 40$ and the time step $\Delta t=0.0025$. Lyapunov vectors were allowed to align for a time span of 35 time units, before the Lyapunov spectrum was calculated for 150 time units according to section 2.2.6.

two exponents should become negative, similar to the pinning effect of the numerical grid observed in figure 3.5 .

However, a parameter scan for $b_{\text {het }}$ and $R$ reveals that the behavior of the system is much richer than expected. Figure 3.12 ) shows the Lyapunov spectrum of the dynamics for a small heterogeneity of $R=1.2$ in model length units, plotted against the parameter $b$ within the heterogeneity. $b_{\text {het }}=0.08$ corresponds to a homogeneous medium. As expected, the two Lyapunov exponents $\lambda_{2}$ and $\lambda_{3}$ indicating spatial symmetry become negative with increasing excitation threshold $b$. The heterogeneity thus seems to create one specific favorable location for the spiral and the "pinning force" to this location increases with the excitation threshold $b_{\text {het }}$ (indicated by the more and more stable symmetry modes) 4 One can already see in the same plot that the destabilization of the meandering modes which was visible for homogeneously increased $b$ (see figure 3.11) is not fully suppressed: $\lambda_{4}$ and $\lambda_{5}$, which were identified as the meandering modes in section 3.1.2, are slightly destabilized, although not enough to lead to a bifurcation and less than in the homogeneous case (see figure 3.11). However, if the heterogeneity is larger, as is the case for figure $3.12 \mathrm{~d})(R=2)$, exactly this happens. Not only is the rate with which the symmetry modes become stable much faster, but at the same time, the meandering modes are destabilized much stronger, leading to a bifurcation close to $b_{\text {het }}=0.115$ which results in one additional zero Lyapunov exponent above $b_{\text {het }}=0.115$ (similar to meandering in a homogeneous medium). Note that, for this to happen, the Lyapunov exponents which correspond to the symmetry and meandering modes have to reverse their order (see value of $b$ marked by "०" in figure $3.12 \mathrm{~d}$ )). This will be discussed in detail at the end of this section. Visual inspection of the spiral tip trajectories confirms that up to $b_{\text {het }}=0.115$, the spiral wave is indeed held in a fixed position by the heterogeneiety, as seen in figure 3.12 a),

\footnotetext{
${ }^{4}$ One could also imagine that the spiral wave is just attracted initially by the heterogeneity but its exact position is still arbitrary, which would result in a preservation of the local symmetry.
} 
a)

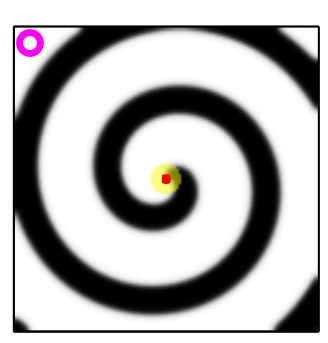

b)

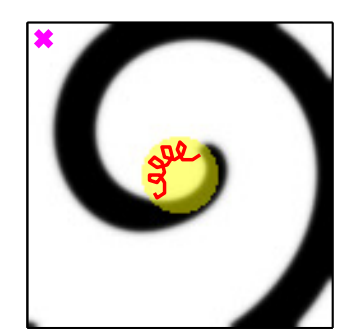

c)

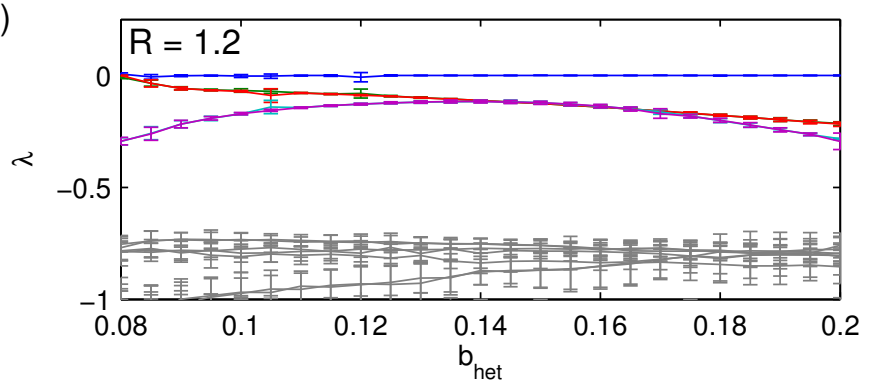

d)
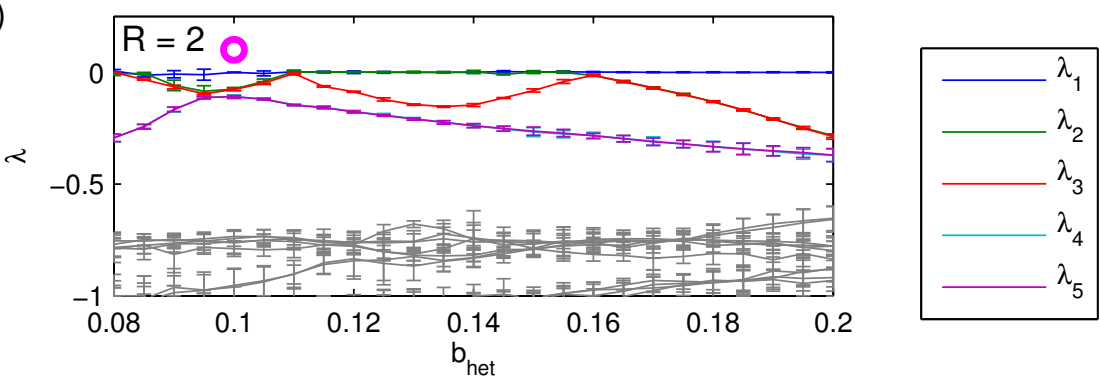

e)

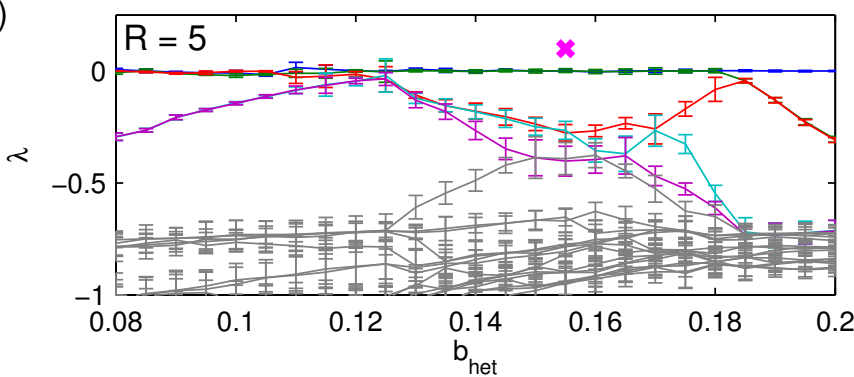

Figure 3.12: Symmetry break by heterogeneity. a), b) Example snapshots of the Barkley model variable $u$ of spirals interacting with a circular heterogeneity of increased excitation threshold $b$ (region indicated by yellow color). Parameter set B4 (see table A.1, page 177) is used with $b=0.08$ throughout the medium, except for the circular region, whose parameter $b_{\text {het }}$ is indicated by "O" and " $\times$ " in d) and e), respectively. The spiral tip path detected using the method outlined in section 2.2 .5 with $\left(u^{*}, v^{*}\right)=(0.48,0.38)$ is plotted as a red line. c) to e) Leading Lyapunov spectra for three different radii of the heterogeneity ( $R=1.2,2,5$, respectively) plotted against the value of the model parameter $b=b_{\text {het }}$ within the circular region. $b_{\text {het }}=0.08$ corresponds to a homogeneous medium. The numerical parameters are identical to those noted in the caption of figure 3.11 . 
whereas above the bifurcation value, the tip starts to meander. This effect is seen more easily for an even larger heterogeneity $(R=5)$, with a Lyapunov spectrum as plotted in figure 3.12 p). The corresponding spiral tip trajectory for the meandering region with two zero Lyapunov exponents is shown in figure 3.12 b).

For small $R$, the heterogeneity therefore acts as a pinning center, despite the fact that a homogeneous medium of the same increased excitation threshold is close to a meandering instability (cf. figure 3.11). For larger $R$, however, this potential instability does not only reappear but is even enhanced, such that an actual bifurcation can take place which is not observed in the homogeneous case. Another interesting phenomenon for $R=5$ is that the symmetry modes are not stabilized for moderate values of $b_{\text {het }}$, indicating that within the heterogeneity, the spiral can still evolve freely if the heterogeneity is not too strong. Also, note that the Lyapunov spectra for moderate $b_{\text {het }}$ (below the bifurcation value) look very similar to the homogeneous case in figure 3.11. The value of $b_{\text {het }}$ at which the spiral wave feels that the modification of $b$ is not global and deviates from the homogeneous behavior therefore increases with increasing $R$. This is consistent with the fact that the case of $R \rightarrow \infty$ is identical to the homogeneous scenario of figure 3.11 .

For a stable spiral wave in a homogeneous medium it was shown in section 3.1 .2 that the leading Lyapunov spectrum consists of three neutrally stable modes which represent a perturbation along the trajectory of the system and spatial shifts in the two available directions due to Euclidian symmetry. The fourth and fifth Lyapunov exponent were associated with the modes which were found to be responsible for the meandering bifurcation considered in section 3.1.4. However, as seen in this section, the relative order of the symmetry and meandering modes in terms of growth rate is not universal, but can be reversed by interaction of the spiral wave with a heterogeneity. In figure $3.12 \mathrm{~d}$ ), it is seen that this reversal can take place when both modes are actually stable, which makes it impossible to differentiate them by observing the dynamics of the spiral wave. By following the change of Lyapunov exponents from the homogeneous case $\left(b_{\text {het }}=0.08\right)$ to the value of $b$ indicated by "O", it was implicitly assumed that the trend in growth rate for both modes continues and that indeed the Lyapunov exponents of the meandering modes approach a value of zero (later confirmed when the bifurcation takes place at about $b_{\text {het }}=0.115$ ). However, since the ordering of Lyapunov exponents from largest to smallest alone defines their indices, one would not be able to follow this (heuristic) reasoning if only the spectrum at $b_{\text {het }}=0.1$ ("○") was known. Fortunately, this is possible making use of one quantity disregarded so far this section: the Lyapunov vectors. Figure 3.13 shows the Lyapunov vectors for the five leading exponents in the system with $R=2$ and $b_{\text {het }}=0.1$. The first one corresponds to the perturbation along the trajectory, whereas the spatial symmetry modes can be found as the fourth and fifth Lyapunov vector. $\delta(u, v)_{4}$ represents a shift of the wave pattern towards the lower right, whereas $\delta(u, v)_{5}$ shifts the spiral wave towards the lower left. In contrast, the second and third Lyapunov vector exhibit a higher-frequency modulation of the wave front characteristic for the meandering modes (see section 3.1.4). In this way, the reversal of stability order necessary for the pinned meandering bifurcation observed in this section can be objectively confirmed. 

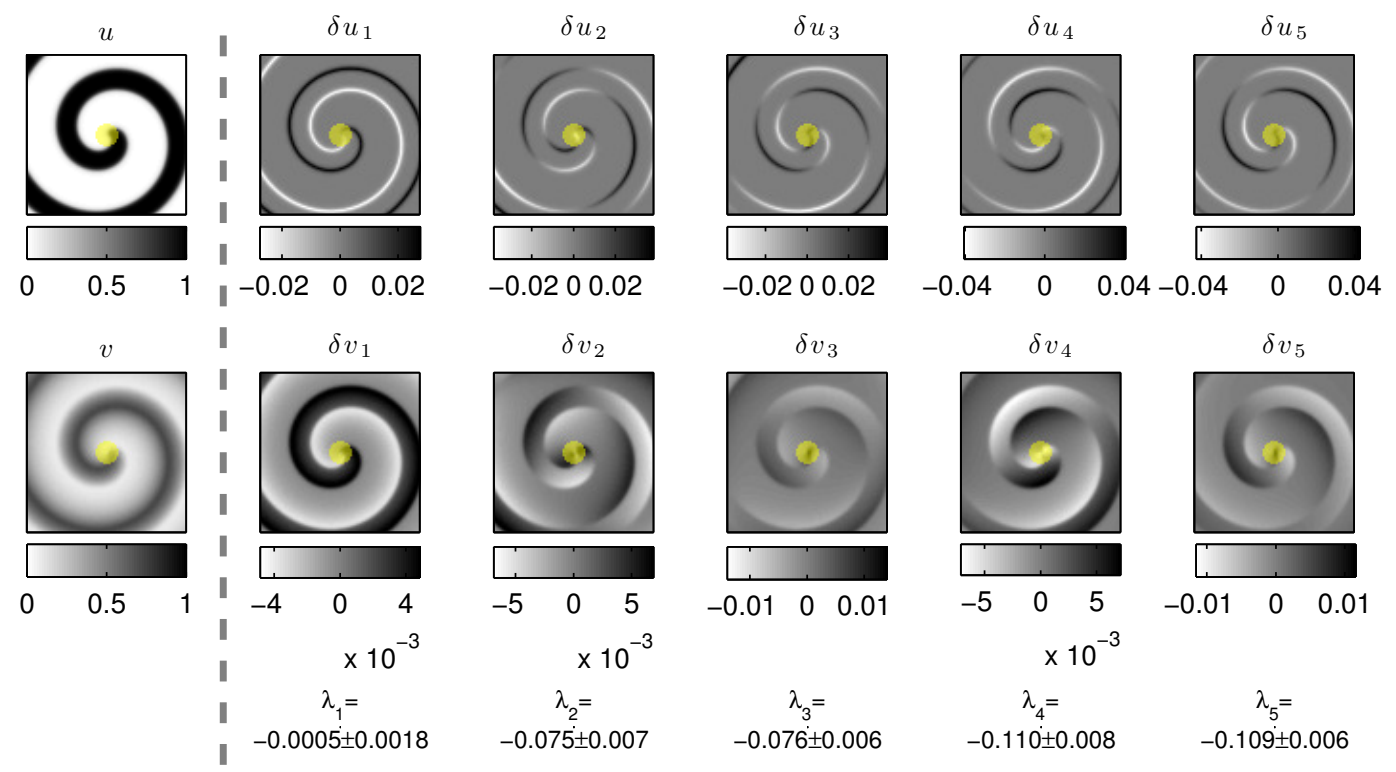

Figure 3.13: Lyapunov vectors of a spiral wave interacting with a circular heterogeneity. Shown are the Lyapunov vectors corresponding to the first five Lyapunov exponents for the parameters indicated by "०" in figure 3.12. The region of increased excitation threshold $b$ is marked by yellow color. The medium state is depicted in the leftmost column. The five columns each show the $u$ and $v$ components of the Lyapunov vectors. Values of Lyapunov exponents are printed below each column.

\subsubsection{Random heterogeneities}

One of the advantages of Lyapunov stability analysis compared to the linear stability analysis of fixed points is that it can be applied to arbitrary trajectories. Therefore, the heterogeneity in the system does not have to be "simple" for the method to work. Thinking about the complex system of the heart and its inherent heterogeneity, not only abrupt changes of tissue properties in localized regions are conceivable, as considered in section 3.1.5. Rather, real-world (and in particular biological) systems are usually subject to natural variability which leads to a random distribution of parameters. Following this line of thoughts, the excitation threshold of the medium is now modified randomly. To compare the results with those in section 3.1.5, random variations of $b$ from the base value of $b_{0}=0.08$ towards higher excitation thresholds are imposed, where a well-defined correlation length serves as a similar measure as the radius of the heterogeneity in section 3.1.5. To construct such a randomly varying $b(x, y)$, so-called Gaussian random fields are utilized. These are fully defined by their auto-correlation function, which makes it particularly easy to obtain different realizations. If $\sigma$ is the intended correlation length, let

$$
R(x, y)=\exp \left(\frac{x^{2}+y^{2}}{2 \sigma^{2}}\right)
$$

be the imposed (spatial) auto-correlation function. Via the Wiener-Khinchin theorem, the Fourier transform of $R$ is the power spectrum of the underlying field $\beta_{\text {full }}(x, y)$, so

$$
\left|\mathcal{F}_{2}\left[\beta_{\text {full }}\right]\right|=\sqrt{\mathcal{F}_{2}[R]}
$$


a)

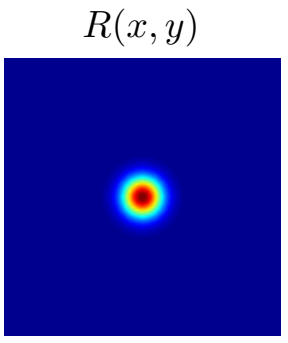

d)

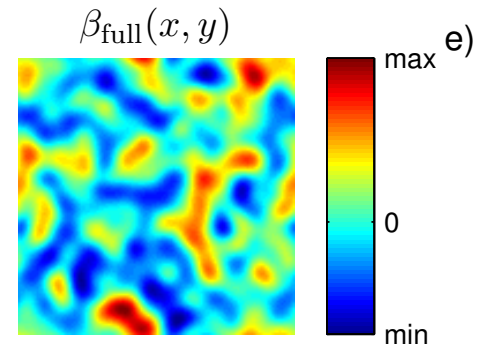

b)

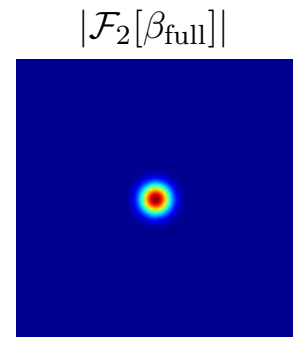

e)

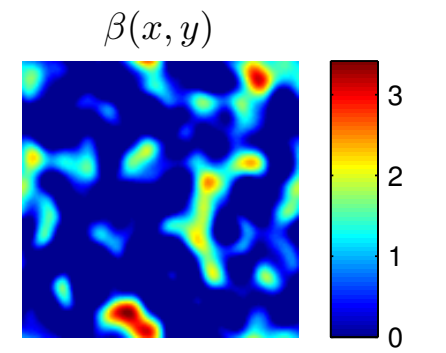

c)

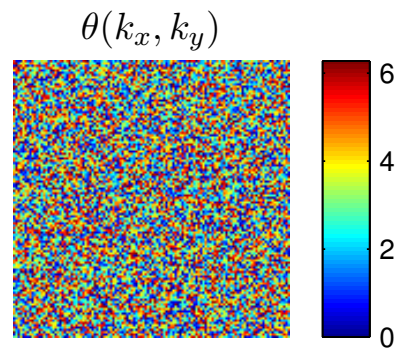

f) $\beta_{2}(x, y)$

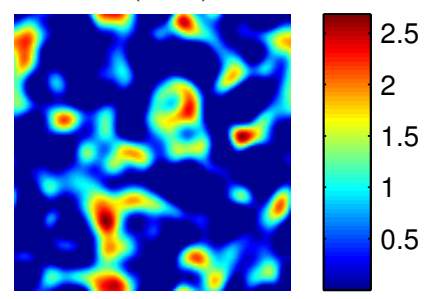

Figure 3.14: Construction of random excitation threshold. a) Imposed twodimensional gaussian auto-correlation function $R(x, y)$ with a defined correlation length $\sigma$ (see equation (3.7)). b) Amplitude of the spectrum of the underlying random field, via Wiener-Khinchin theorem from a) as in equation (3.8). c) Random phase $\theta(x, y)$ determining the realization of the field. d) Realization of the field according to equation (3.9). e) Normalized and capped version $\beta(x, y)$ of the field in d) used to modify the excitation threshold. f) Same as e), but for a different realization, i.e. different phases in c).

where $\mathcal{F}_{2}$ denotes the two-dimensional Fourier transform in space. As the Fourier transform of the field $\beta_{\text {full }}$ is only determined up to its amplitude by this relation, different realizations of the field can be obtained by choosing random phases $\theta\left(k_{x}, k_{y}\right)$ for each mode (numerically: for each computational point) and transforming the spectrum back to real space:

$$
\beta_{\text {full }}=\operatorname{Re}\left(\mathcal{F}_{2}^{-1}\left[\theta \cdot \sqrt{\mathcal{F}_{2}[R]}\right]\right),
$$

where Re is the real part. The standard deviation of the obtained realization $\beta_{\text {full }}(x, y)$ across the whole domain is then normalized to one and negative regions are set to zero to give the final modulation $\beta(x, y)$ :

$$
\beta(x, y)=\frac{1}{\operatorname{std}\left(\beta_{\text {full }}\right)}\left\{\begin{array}{ll}
0 & \beta_{\text {full }}(x, y)<0 \\
\beta_{\text {full }}(x, y) & \beta_{\text {full }}(x, y)>0
\end{array} \quad \forall x, y\right.
$$

The complete process of construction is illustrated in figure 3.14 . The field $b(x, y)$ for the simulation is then defined as

$$
b(x, y)=b_{0}+\left(b_{\text {target }}-b_{0}\right) \beta(x, y) \quad \forall x, y
$$

The number $b_{\text {target }}$ is by construction the value of $b$ which is one standard deviation of $\beta_{\text {full }}$ above the base value $b_{0}$ of the excitation threshold. It serves here as a measure for the amplitude of the heterogeneity and has a meaning similar to $b_{\text {het }}$ in section 3.1.5. Simulations for a range of correlation lengths $\sigma$ and target excitabilities $b_{\text {target }}$ were carried 
out. Because of the stochasticity of the system, for every fixed $\sigma$ and $b_{\text {target }}$ Lyapunov exponents were calculated using 40 different realizations of $b(x, y)$. The ensemble averages for three selected values of $\sigma$ along with two exemplary snapshots are shown in figure 3.15 . For rather small-scale heterogeneities $(\sigma=0.71)$, figure 3.15; ) shows that little more happens for increasing $b_{\text {target }}$ than the gradual stabilization of the Euclidian symmetry modes. This is in a way expected, because the spiral wave interacts with the first heterogeneity it encounters and pins to it. The gradual effect is a result of an increasing average amplitude of the heterogeneity with increasing $b_{\text {target }}$. The meandering modes are only slightly destabilized and Lyapunov exponents with even higher indices, $\lambda_{i}, i>5$ (shown in gray), only increase for the extreme values of $b_{\text {target }}$. Note also that the standard deviation of the ensemble Lyapunov exponents increases towards higher $b_{\text {target }}$, because there is a larger variability in the amplitude of heterogeneities the spiral can encounter.

For heterogeneities on larger spatial scales $(\sigma=1.56)$, figure $3.15 \mathrm{~d})$ shows a similar behavior. However, the symmetry modes are stabilized more rapidly and the meandering modes are considerably more destabilized compared to the first case. Thus, although there is, on average, no bifurcation, some of the behavior for a discrete heterogeneity (cf. figure 3.12) is maintained. A prominent difference is seen in the higher-index Lyapunov exponents, which clearly increase for higher $b_{\text {target }}$, closing the otherwise large gap below the meandering modes. This is probably due to the heterogeneities with which the spiral tip is not directly interacting and which are distributed across the rest of the medium - a feature that does not exist for the discrete heterogeneities of section 3.1.5.

Figure 3.15 ) indicates that all these features are preserved for even larger heterogeneities $(\sigma=2.97)$, except for one: for small $b_{\text {target }}$, the pinning force (as measured by the stabilization of the symmetry modes) stays relatively weak, before intensifying from $b_{\text {target }} \approx 0.11$ upwards. This is again a somewhat masked version of the effect seen for large heterogeneities in figure 3.12 ): There, no symmetry break was observed up to a certain threshold value of the heterogeneity amplitude. Hence, even for the random case, the larger the typical size of the heterogeneities, the stronger this heterogeneity needs to be to cause a considerable pinning effect, although the meandering modes are still destabilized immediately. The latter could make the spiral wave prone to other destabilizing factors, like a global parameter shift of the medium or random stimuli.

It should be emphasized that all the Lyapunov spectra considered in this section were ensemble averages, although it was confirmed that no meandering is observed for any single realization of heterogeneity. Nevertheless, it would be interesting to find out, whether the reversal of the growth rates for symmetry and meandering modes as observed in section 3.1 .5 can similarly occur in this generalized scenario. Unfortunately, the Lyapunov vectors which turned out to be very useful for circular heterogeneities are less indicative in this case. The first five Lyapunov vectors for the system whose parameters are marked by " $\times$ " in figure 3.15 are shown in figure 3.16. While the first Lyapunov vector can clearly be identified as a perturbation along the trajectory, the next four Lyapunov vectors appear to be more or less random modulations of the wave front with no discernible regularity. This is likely to be due to the irregular propagation of the spiral wave, which causes fluctuating growth rates of which the Lyapunov exponents (which nevertheless have an exact two-fold degeneracy) are only averages. Thus, the Lyapunov vectors are also time-dependent beyond a simple rotation. 
a)

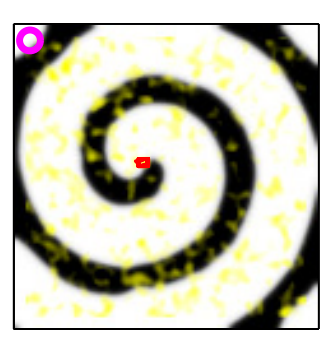

b)

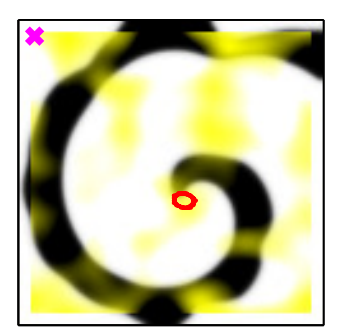

c)

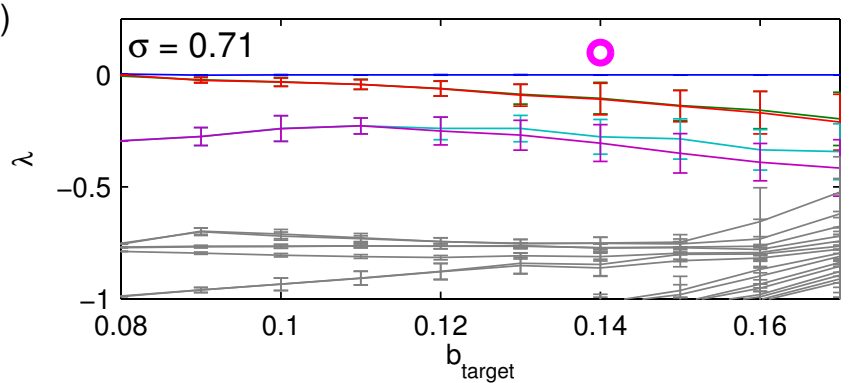

d)
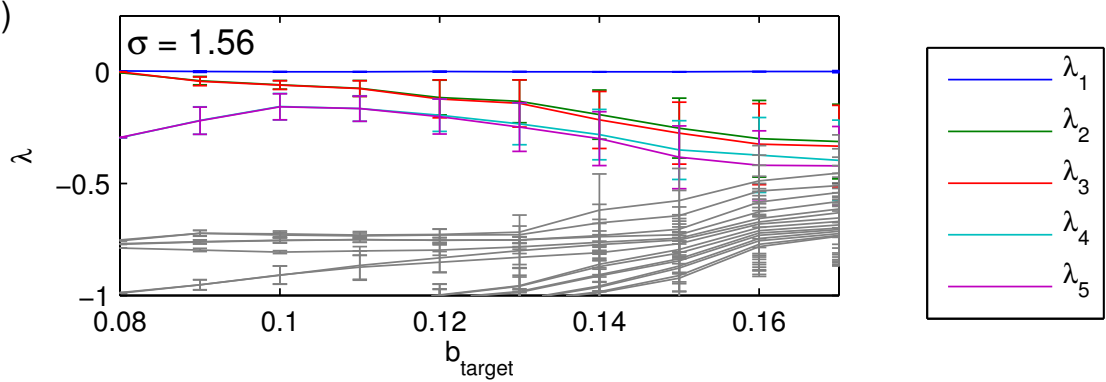

e)

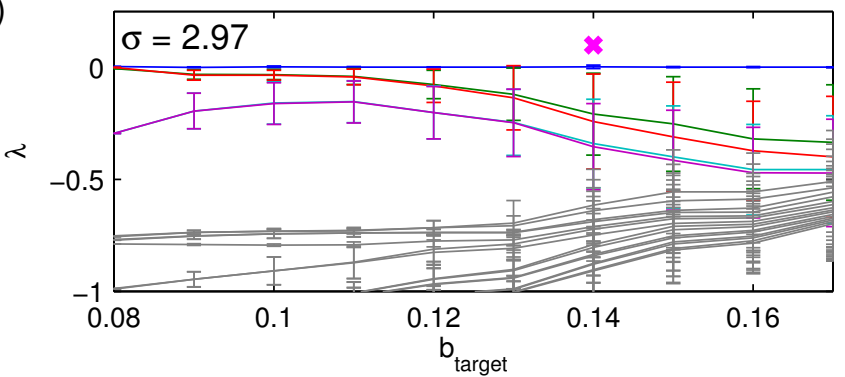

Figure 3.15: Symmetry break by random heterogeneity. a), b) Example snapshots of the Barkley model variable $u$ of spirals interacting with the random heterogeneity of increased excitation threshold $b$ (deviation $b(x, y)-b_{0}$ from the base value indicated by yellow color). Base parameters correspond to parameter set B4 (see table A.1, page 177) with $b_{0}=0.08$. The value of $b_{\text {target }}$ for the example snapshots is indicated by "o" and " $\times$ " in c) and e), respectively. The spiral tip path detected using the method outlined in section 2.2.5 with $\left(u^{*}, v^{*}\right)=(0.48,0.38)$ is plotted as a red line. c) to e) Leading Lyapunov spectra for three different correlation lengths $\sigma$ plotted against the strength $b_{\text {target }}$ of the heterogeneity. $b_{\text {target }}=0.08$ corresponds to a homogeneous medium. The plotted Lyapunov exponents are ensemble averages, error bars indicate standard deviation. The numerical space step is $h=1 / 3$, the domain size $40 \times 40$ and the time step $\Delta t=0.0025$. Lyapunov vectors were allowed to align for a time span of 60 time units, before the Lyapunov spectrum was calculated for 525 time units according to section 2.2.6. 

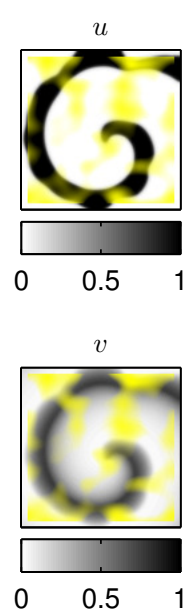
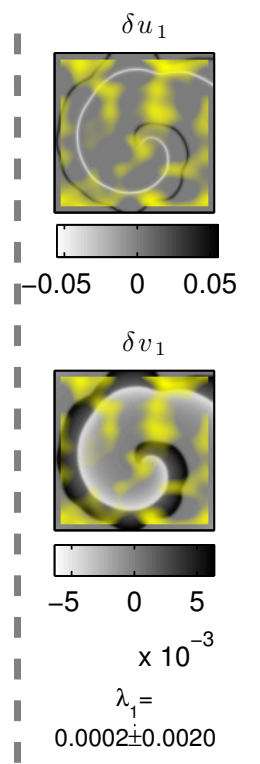
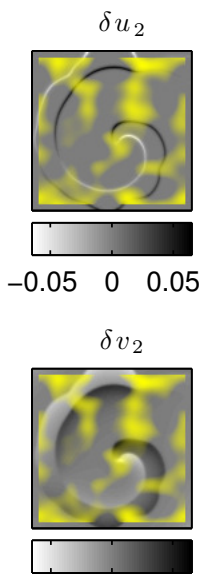

$\begin{array}{lll}-0.01 & 0 & 0.01\end{array}$

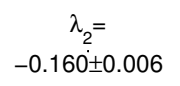

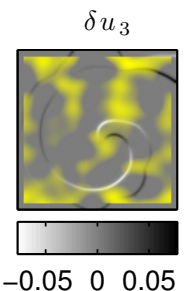
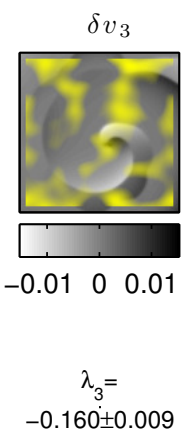

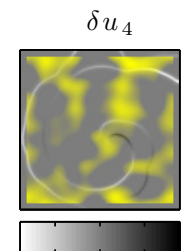

$-0.0500 .05$
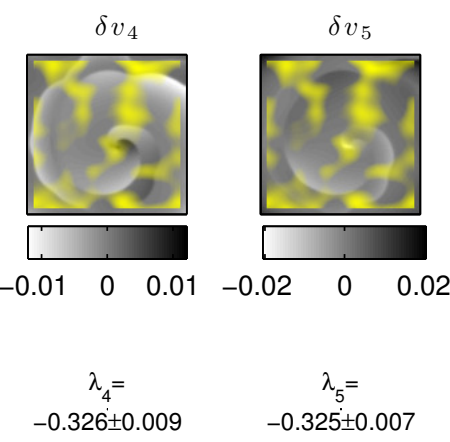

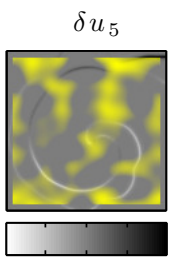

$-0.0500 .05$

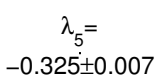

Figure 3.16: Lyapunov vectors of a spiral wave interacting with random heterogeneity. Shown are the Lyapunov vectors corresponding to the first five Lyapunov exponents for the parameters indicated by " $\times$ " in figure 3.15 . The deviation $b(x, y)-b_{0}$ from the base value is indicated by yellow color. The medium state is depicted in the leftmost column. The five columns each show the $u$ and $v$ components of the Lyapunov vectors. Values of Lyapunov exponents are printed below each column.

In summary, the above analysis shows that the interpretation of the Lyapunov spectrum for this specific case does not change dramatically when making the transition more realistic and asymmetric forms of heterogeneity. Considering the global nature of this heterogeneity (as opposed to the localized disk in section 3.1.5), this implies that the modes with a Lyapunov exponent close to zero (for the homogeneous case) mainly respond to the interactions located at the spiral center. This is in good agreement with the localization of response functions in a region close to the spiral tip [81, 109]. Only for large and strong heterogeneities, the outer regions of the spiral wave become important, leading to a destabilization of the lower Lyapunov spectrum. This is intuitive to some extent, because these heterogeneities might eventually cause breakup in the waves sweeping over them. The fact that no meandering was observed for random heterogeneities suggests that the sharp drop of the excitation threshold for the single, isolated heterogeneity considered in section 3.1 .5 is a necessary prerequisite for this effect.

\subsubsection{Heterogeneities in spatio-temporal chaos}

In sections 3.1.5 and 3.1.6, the interaction of a single spiral wave with heterogeneities of the medium parameters was examined. As outlined in the introduction (see section 1.4), spiral waves underlie tachycardias in the heart and their stability properties are of major interest because they may degenerate into a life-threatening state of spatio-temporal chaos. It is reasonable to assume that the chances of terminating such turbulent activity with gentle defibrillation methods depend on the complexity (or chaoticity) of the dynamics and that this complexity depends on the properties of the tissue and the degree of heterogeneity in the system. However, different consequences of heterogeneity are conceivable: On the 
one hand, it could increase complexity, because it adds diversity to the substrate. On the other hand, it could decrease complexity due to the additional spatial structure imposed. The third and probably most plausible possibility is that, assuming the whole range of heterogeneous parameters gives rise to spatio-temporal chaos, the chaotic dynamics leads to an average complexity in between those observed for homogeneous systems within the same range of parameters. Therefore, the subject of this last topic of section 3.1 is the question, how spatial heterogeneities can alter the dynamics during spatio-temporal chaos beyond what can be inferred from individual homogeneous systems with different parameters.

For chaotic attractors, Lyapunov stability analysis provides a means to quantitatively assess complexity, namely the Lyapunov dimension $D_{\mathrm{L}}$, which was introduced by J. A. KAPlan and J. L. Yorke [216]. It was conjectured by KAPLAN and Yorke that for typical (non-pathological) systems, $D_{\mathrm{L}}$ is equal to the Rényi dimension of order 1 [217], which is also called the information dimension and is a fractal dimension for the attractor in phase space. A direct calculation of the information dimension is almost impossible for very high-dimensional attractors, as it relies on the estimation of the asymptotic probability distribution of trajectories in phase space and the time until densities sufficient for this estimation are reached increases with increasing dimensionality. $D_{\mathrm{L}}$, however, is a function of the spectrum of Lyapunov exponents and can thus easily be calculated, if the Lyapunov exponents are known. It is defined as

$$
D_{\mathrm{L}}=k+\frac{1}{\left|\lambda_{k+1}\right|} \underbrace{\sum_{i=1}^{k} \lambda_{i}}_{*}
$$

where $k$ is the largest index such that the sum $(*)$ is still non-negative. Consequently, for non-chaotic attractors with $\lambda_{i} \leq 0$ for all $i$, the Lyapunov dimension $D_{L}$ is equal to the number of Lyapunov exponents which are equal to zero. Heuristically, in the general case, $D_{\mathrm{L}}$ is the number of degrees of freedom necessary to describe the dynamics, such that the resulting set in phase space is, on average, neither expanding nor contracting. For this reason, it can be used to estimate an effective number of degrees of freedom for high- or infinite-dimensional systems. In fact, as noted in the introduction in section 1.7, in one of the two studies which have made use of Lyapunov exponents in excitable media so far, M. C. Strain and H. S. Greenside did exactly that [121]: In the Bär-Eiswrith model during spatio-temporal chaos, $D_{\mathrm{L}}$ was found to be an extensive quantity and the average number of degrees of freedom per phase singularity was found to be a function of the model parameter $\epsilon$ in the chaotic regime. The following results build on this knowledge by considering heterogeneities in the same model parameter.

Simulations are carried out in a medium of size $40 \times 40$ with periodic boundary conditions, using the Bär-Eiswirth model with parameters $a=0.84, b=0.07$ and $\epsilon$ varying between 0.08 and 0.12 . For the whole range of $\epsilon$, spiral waves are known to break up leading to a state of spatio-temporal chaos [73]. In all cases, the activity is initiated by creating two spiral waves which subsequently break up as illustrated in figure 3.17a). Note that the initial condition is slightly asymmetric to prevent mirror symmetry which would persist even after breakup. The simulation is allowed to equilibrate for 1500 model time units 
a)

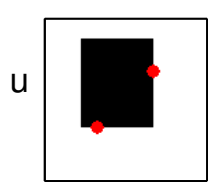

$$
t=0
$$

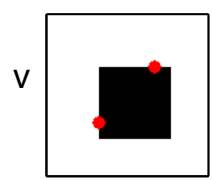

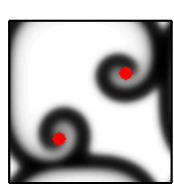

$\mathrm{t}=7$

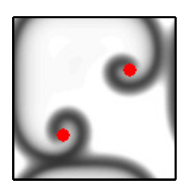

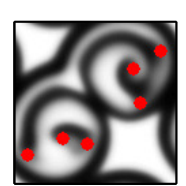

$t=14$

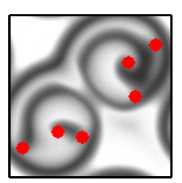

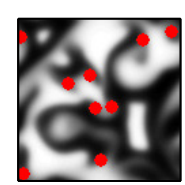

$\mathrm{t}=21$

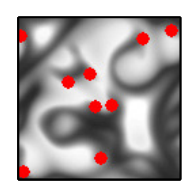

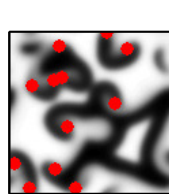

$t=28$

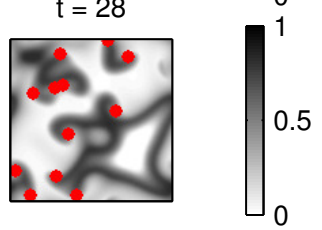

b)
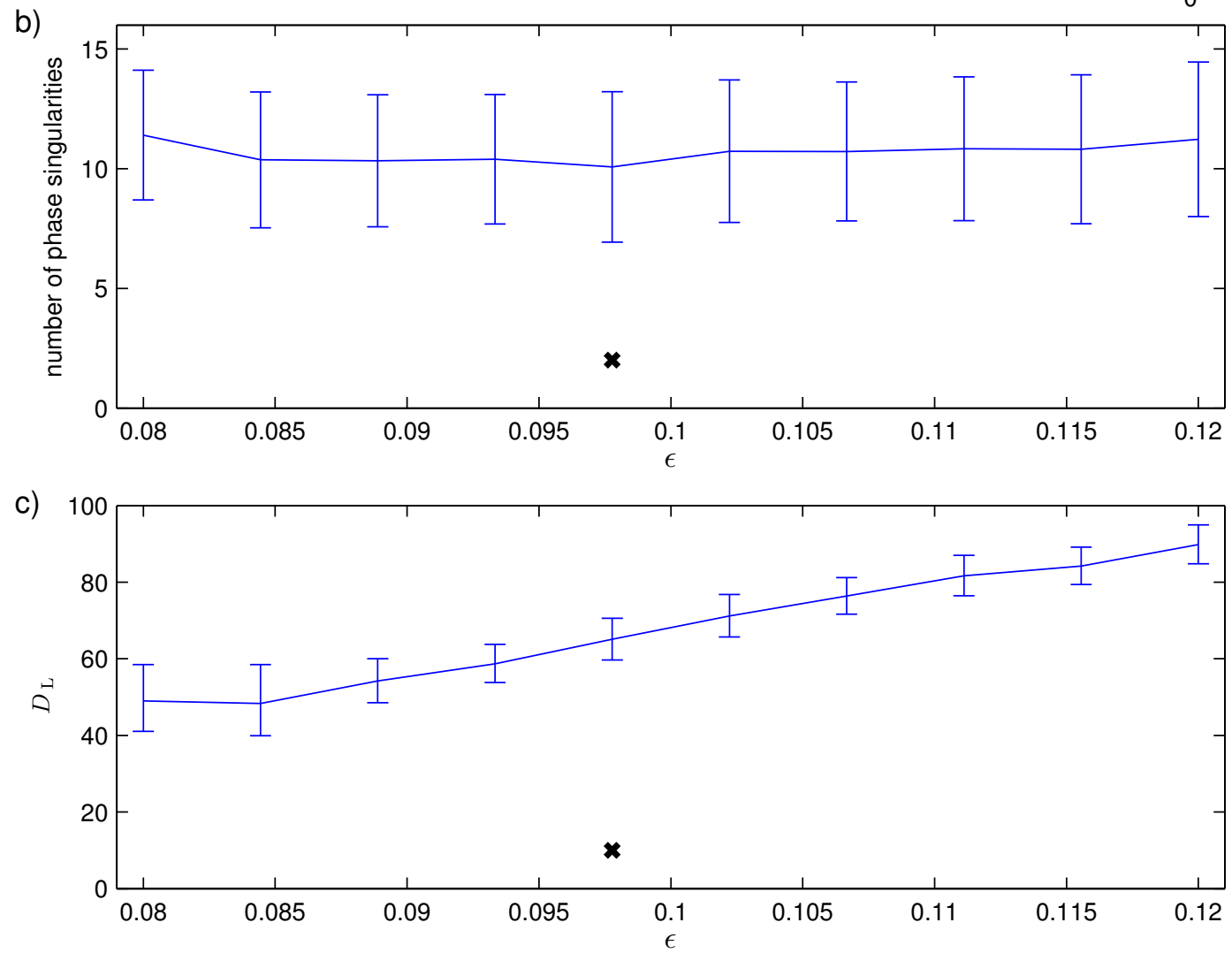

Figure 3.17: Spatio-temporal chaos in the Bär-Eiswrith model. a) Snapshots of the model variables $u$ and $v$ for the beginning of an exemplary simulation showing the initiation protocol for spatio-temporal chaos (time progresses from left to right). Two spiral waves quickly break up and a turbulent state is formed. Red dots correspond to phase singularities detected by the method outlined in section 2.2 .5 with $\left(u^{*}, v^{*}\right)=(0.65,0.45)$. The value of $\epsilon$ is marked by " $\times$ " in b) and c). b) Mean number of phase singularities in simulations exhibiting spatio-temporal chaos, initiated as in a), using parameter set BE1 (see table A.1, page 177) with different values of $\epsilon$. The number of phase singularities is calculated every 1 model time units from $t=1500$ to $t=3000$ to allow for equilibration. Error bars indicate standard deviation. c) Kaplan-Yorke dimension $D_{\mathrm{L}}$ for the same parameters as in b). Error bars indicate the possible range of $D_{\mathrm{L}}$ for Lyapunov spectra shifted by the error as calculated according to section 2.2.6. The numerical space step is $h=1 / 3$, the domain size $40 \times 40$ and the time step $\Delta t=0.002$. Lyapunov vectors were allowed to align for a time span of 200 time units, before the Lyapunov spectrum was calculated for 1500 time units (roughly 300 dominant periods) according to section 2.2.6. 
(roughly 300 dominant periods) before Lyapunov exponents and phase singularity statistics are obtained for another 1500 time units. Phase singularities are detected every 1 time units using the method introduced in section 2.1.7 with $\left(u^{*}, v^{*}\right)=(0.65,0.45)$. In contrast to reference [121], this fixed definition of phase is used independent of the value of $\epsilon$, to be able to obtain comparable results later in the heterogeneous case. The Lyapunov dimension $D_{\mathrm{L}}$ is calculated for the obtained Lyapunov spectra according to equation (3.12). In order to assess the accuracy of the dimension estimation, the calculated Lyapunov spectrum is shifted up (down) by the error estimate introduced in section 2.2.6 and the Lyapunov dimension is calculated for the shifted spectra to yield an upper (lower) bound for $D_{\mathrm{L}}$.

Figure 3.17b) shows that, in the parameter range considered here, neither the number nor the number fluctuation of phase singularities depends strongly on the parameter $\epsilon 5^{5}$ Roughly 10 phase singularities are present on average in the simulation domain. In contrast, as indicated by figure 3.17k), the Lyapunov dimension $D_{\mathrm{L}}$ increases steadily with increasing $\epsilon$. This is plausible, as the transition to spiral breakup occurs just below $\epsilon=0.07$ [73] and is consistent with the results reported in reference [121]. Beyond the critical value, further increase of $\epsilon$ thus increases the chaoticity of the system.

What happens now if different values of $\epsilon$ from this range are combined in a single medium? Similarly to the results presented in section 3.1.5. circular heterogneities are considered. Since multiple spiral waves are present in the system, not only one but several circular heterogeneities, in which the value of $\epsilon$ is modified, are inserted into the system. For simplicity, here, a regular grid of $4 \times 4$ heterogeneities is chosen, such that the total number of 16 heterogeneities is on the same order as the average number of phase singularities as calculated in figure $3.17 \mathrm{p}$ ). Simulations were carried out for three different levels $\epsilon_{\text {het }}$ within the circular regions, whose radius radii $R$ was also varied. In the rest of the medium, $\epsilon$ was kept at its base value of 0.08 . Phase singularity statistics and the Lyapunov dimension $D_{\mathrm{L}}$ are calculated as above. Snapshots of an examplary simulation for heterogeneities with $\epsilon_{\text {het }}=0.12$ and $R=3$ and the time evolution of the number of phase singularities can be found in figure 3.18 .

The statistical results for all simulations are summarized in figure 3.19. The number of phase singularities shown in figure 3.19 a) deviates only slightly from the expected behavior: the mean number of phase singularities is approximately uninfluenced by the heterogeneity for all three levels of $\epsilon_{\text {het }}$, which is consistent with the fact that changes in $\epsilon$ in the considered range do not in general alter this quantity (cf. figure 3.17b)). Only for radii around $R=3.5$, the number of phase singularities is slightly reduced for $\epsilon_{\text {het }}=0.107$ and the smaller errors bars indicate that heterogeneities with $\epsilon_{\text {het }}=0.107$ and $\epsilon_{\text {het }}=0.12$ cause a decrease in the number fluctuations of phase singularities. What comes as a surprise is the effect of the heterogeneity on the complexity of the chaotic dynamics. Figure $3.19 \mathrm{~b}$ ) shows that $D_{\mathrm{L}}$ is more or less constant up to a certain radius of the heterogeneity for all $\epsilon_{\text {het }}$. This is remarkable, since, for increasing $R$, a growing fraction of the domain has parameters which should cause an increase in complexity according to figure $3.17 \mathrm{k}$ ). Even

\footnotetext{
${ }^{5}$ It should be emphasized that the number of phase singularities which are detected depends on the chosen origin $\left(u^{*}, v^{*}\right)$ necessary to define the phase. Although visual inspection of the dynamics for the parameters considered in this section confirms that the detected phase singularities are reasonable, the numbers can deviate from those reported in reference [121.
} 
a)

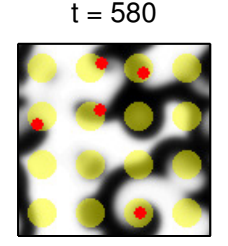

$t=1580$

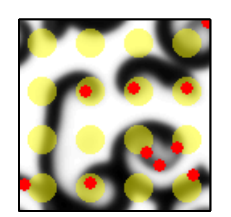

$\mathrm{t}=780$

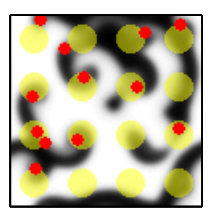

$t=1780$

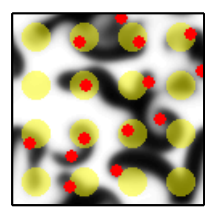

$\mathrm{t}=980$

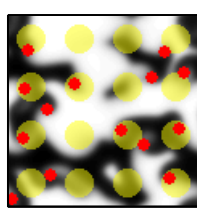

$t=1980$

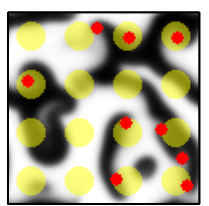

$\mathrm{t}=1180$

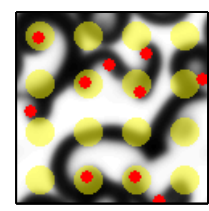

$\mathrm{t}=2180$

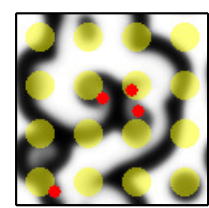

$\mathrm{t}=1380$

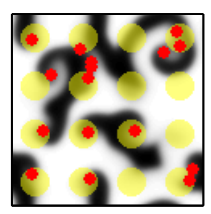

$\mathrm{t}=2380$

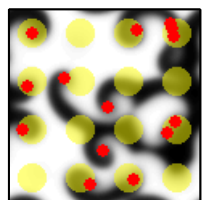

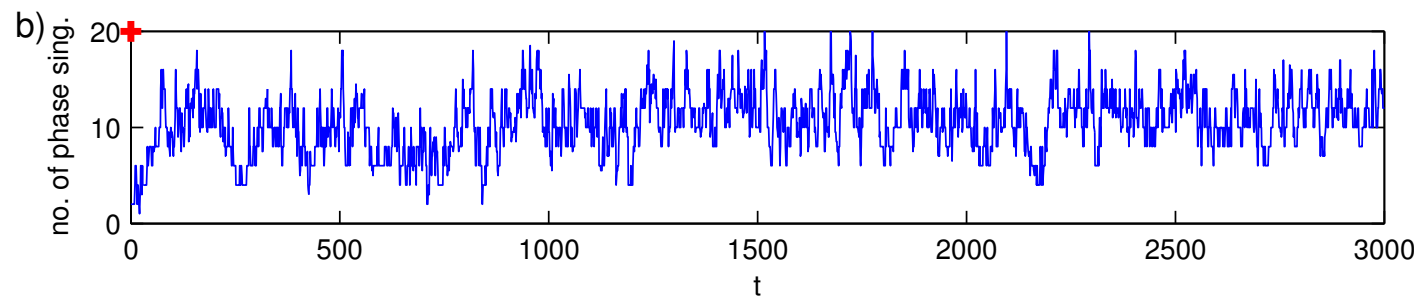

Figure 3.18: Spatio-temporal chaos in a heterogeneous system. a) Example snapshots of the Bär-Eiswirth model variable $u$ of spatio-temporal chaos interacting with circular heterogeneities, within which $\epsilon$ is increased to $\epsilon_{\text {het }}$ (indicated by yellow color). Red dots correspond to phase singularities detected by the method outlined in section 2.2 .5 with $\left(u^{*}, v^{*}\right)=(0.65,0.45)$. The radius $R=3$ of the heterogeneities and $\epsilon_{\text {het }}=0.12$ are indicated by "+" in figure 3.19. Parameter set BE1 (see table A.1, page 177) is used; outside the yellow regions $\epsilon=0.08$. b) Number of phase singularities for the entire simulation time. Statistics (shown in figure 3.19) are calculated from $t=1500$ onwards. The "+" at $t=0$ indicates that the data corresponds to the equally marked parameters in figure 3.19 . 
a)

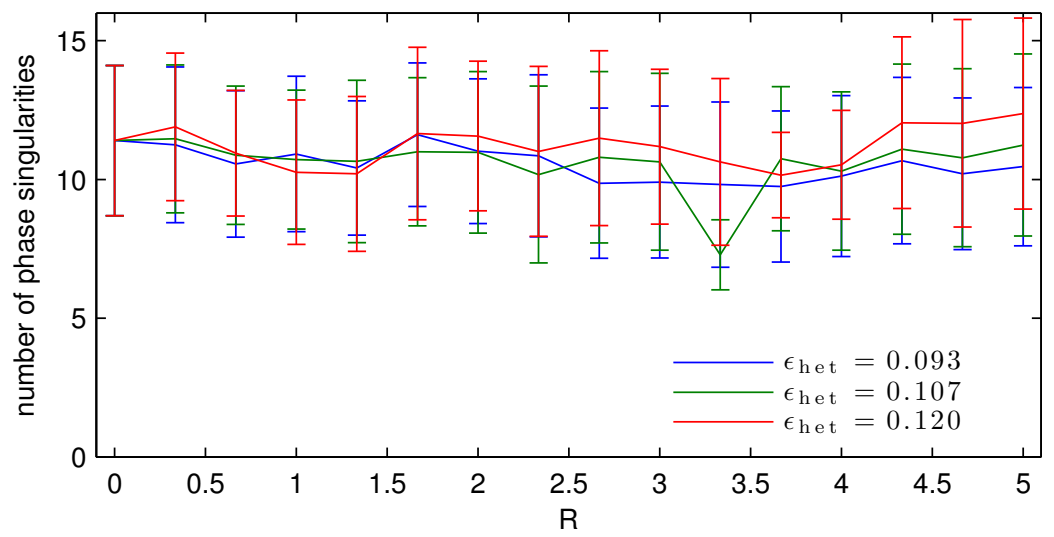

b)

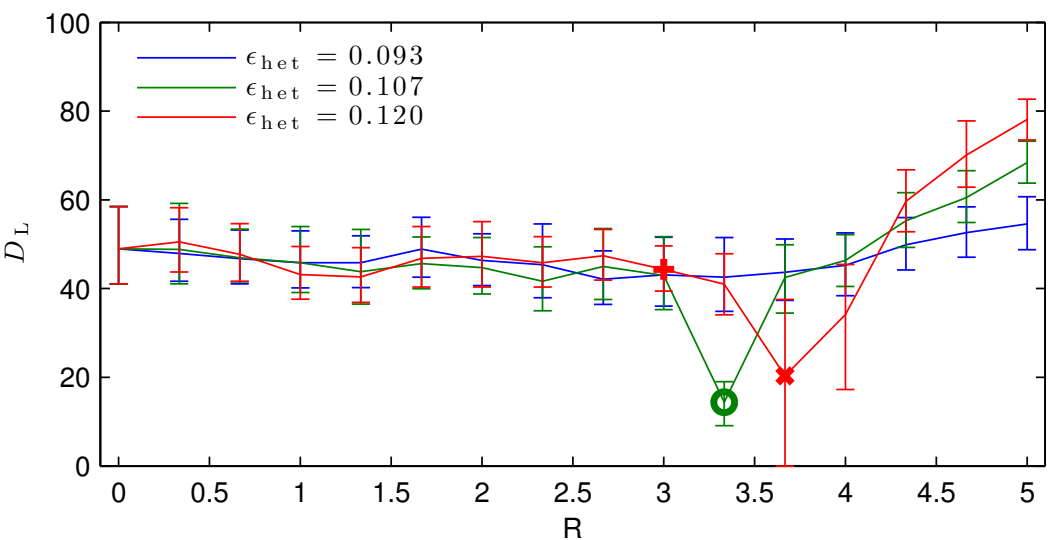

Figure 3.19: Complexity of chaos in a heterogeneous system. a) Mean and standard deviation of the number of phase singularities (calculated as in figure 3.17) plotted against the radius $R$ of the heterogeneities for different values of $\epsilon_{\text {het }}$. b) Kaplan-Yorke dimension $D_{\mathrm{L}}$ for the same systems as in a). Error bars have the same meaning as in figure 3.17 . Symbols "+", "○" and " $\times$ " mark the parameters $\epsilon_{\text {het }}$ and $R$ of the dynamics displayed in figures 3.18, 3.20 and 3.21, Parameter set BE1 (see table A.1, page 177) is used; outside the heterogeneities $\epsilon=0.08$. Numerical parameters are identical to those indicated in the caption of figure 3.17 . 
a)

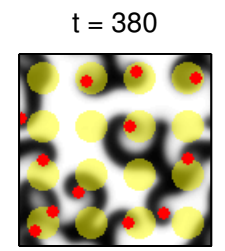

$t=1980$

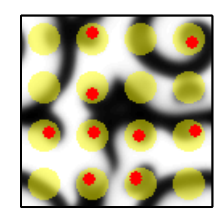

$t=420$

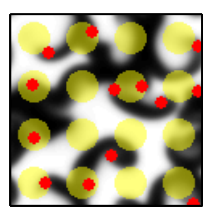

$t=2180$

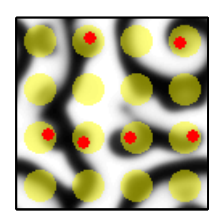

$t=460$

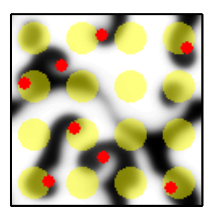

$t=2380$

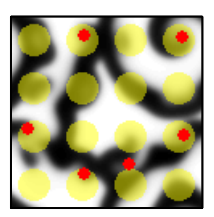

$t=500$

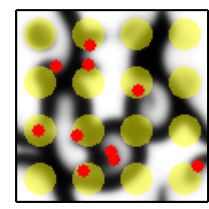

$t=2580$

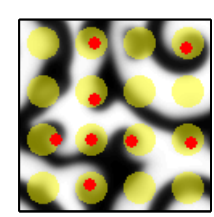

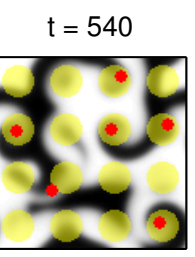

$t=2780$

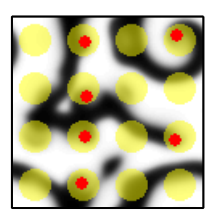

b)

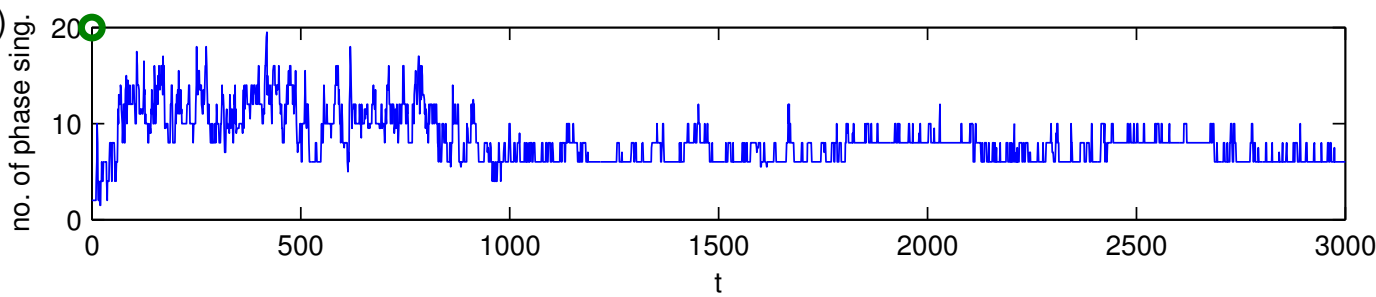

Figure 3.20: Spatio-temporal chaos in a heterogeneous system. a) Example snapshots of the Bär-Eiswirth model variable $u$ of spatio-temporal chaos interacting with circular heterogeneities, within which $\epsilon$ is increased to $\epsilon_{\text {het }}$ (indicated by yellow color). Red dots correspond to phase singularities detected by the method outlined in section 2.2.5 with $\left(u^{*}, v^{*}\right)=(0.65,0.45)$. The radius $R=3.33$ of the heterogeneities and $\epsilon_{\text {het }}=0.107$ are indicated by "○" in figure 3.19. Parameter set BE1 (see table A.1, page 177) is used; outside the yellow regions $\epsilon=0.08$. b) Number of phase singularities for the entire simulation time. Statistics (shown in figure 3.19) are calculated from $t=1500$ onwards. The " $\bigcirc$ " at $t=0$ indicates that the data corresponds to the equally marked parameters in figure 3.19 .

for $\epsilon_{\text {het }}=0.12$, which, in the homogeneous case, doubles $D_{\mathrm{L}}$ with respect to $\epsilon=0.08$, no effect at all is seen. Of course, for $R \rightarrow \infty$, the homogeneous behavior should be recovered, which is why $D_{\mathrm{L}}$ eventually increases towards $R=5$ (at this radius, the heterogeneities just touch each other). However, before these large values of $R$ are reached, $D_{\mathrm{L}}$ even decreases below the values of a homogeneous medium. This drastic reduction in complexity occurs at different values for $\epsilon_{\text {het }}=0.107$ and $\epsilon_{\text {het }}=0.12$, although this could also be a statistical effect.

For $\epsilon_{\text {het }}=0.107$, the dynamics for this minimum complexity is illustrated in figure 3.20 . After an initial phase of very turbulent spiral wave breakup, during which the locations of phase singularities have no or little correlation with the heterogeneities, the fluctuation of phase singularity number is reduced (for $t>1000$ ) and the system merely switches between different spatial arrangments of phase singularities trapped by heterogeneities. For $\epsilon_{\text {het }}=0.12$, the large error bar of $D_{\mathrm{L}}$ for the minimum complexity in figure $3.19 \mathrm{~b}$ ) indicates that dynamics qualitatively changed during the extraction of the Lyapunov spectrum. Indeed, after a transient phase of spiral wave breakup, the phase singularities settle down in the regions of increased $\epsilon$ and are trapped for the rest of the simulation as depicted in figure 3.21. For both values of $\epsilon_{\text {het }}$, the (transient or seemingly permanent) pinning of heterogeneities also explains the reduced phase singularity number fluctuations indicated 
a)

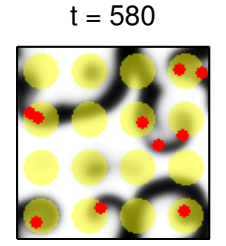

$t=1580$

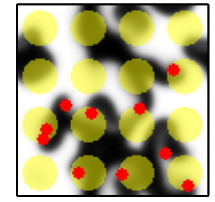

$\mathrm{t}=780$

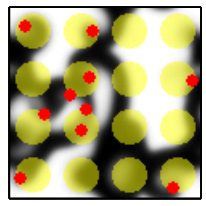

$\mathrm{t}=1780$

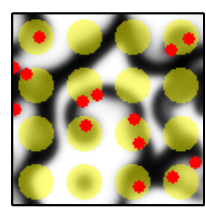

$t=980$

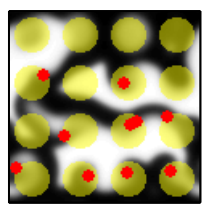

$t=1980$

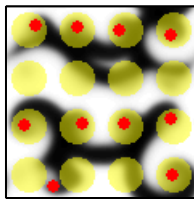

$t=1180$

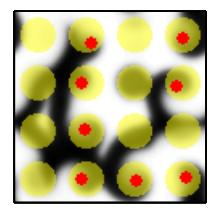

$t=2180$

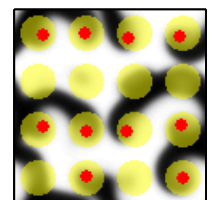

$t=1380$

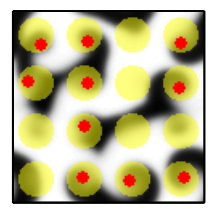

$t=2380$

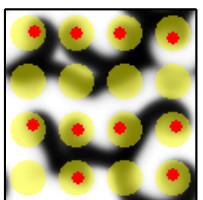

\section{b)}

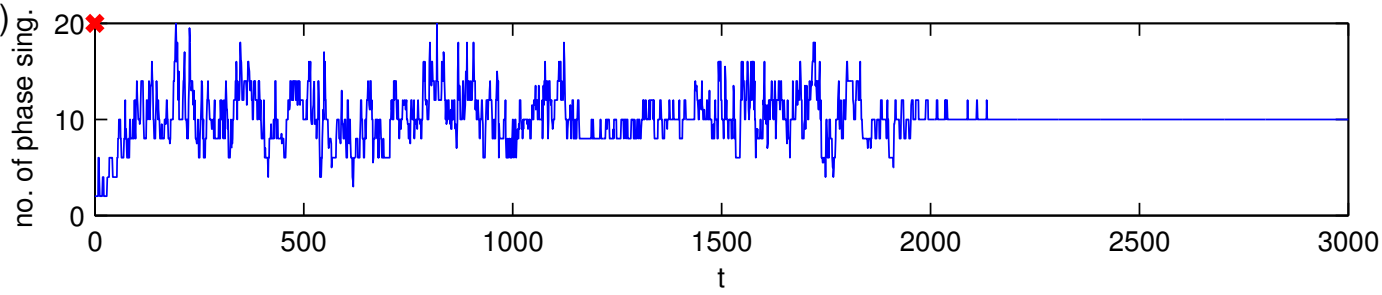

Figure 3.21: Spatio-temporal chaos in a heterogeneous system. a) Example snapshots of the Bär-Eiswirth model variable $u$ of spatio-temporal chaos interacting with circular heterogeneities, within which $\epsilon$ is increased to $\epsilon_{\text {het }}$ (indicated by yellow color). Red dots correspond to phase singularities detected by the method outlined in section 2.2.5 with $\left(u^{*}, v^{*}\right)=(0.65,0.45)$. The radius $R=3.67$ of the heterogeneities and $\epsilon_{\text {het }}=0.12$ are indicated by " $\times$ " in figure 3.19. Parameter set BE1 (see table A.1, page 177) is used; outside the yellow regions $\epsilon=0.08$. b) Number of phase singularities for the entire simulation time. Statistics (shown in figure 3.19) are calculated from $t=1500$ onwards. The " $\times$ " at $t=0$ indicates that the data corresponds to the equally marked parameters in figure 3.19 . 
by figure 3.19 ) for those values of $R$ and $\epsilon_{\text {het }}$.

The observed, and at first sight counter-intuitive, behavior of the phase singularities and the complexity measure $D_{\mathrm{L}}$ becomes intelligible, if one considers the results of section 3.1.5. There, it was shown that localized regions of reduced excitability can stabilize the symmetry modes of a spiral wave, which, in the context of this section, also corresponds to a dimension reduction of the attractor. The parameter $\epsilon$ varied in this section has a similar effect: although a larger $\epsilon$ increases the complexity of the dynamics as seen in figure 3.17, at the same time, it reduces the excitability of the system (cf. equation 2.38). Therefore, the heterogeneities exert an attracting force on the spiral waves present during spatio-temporal chaos and thereby restrict the dynamics, counteracting the inherent destabilizing effect of their local parameters. Interestingly, for a large range of radii $R \lesssim 3$, this effect is very subtle, such that the number of phase singularities as well as $D_{\mathrm{L}}$ do not indicate any change to the system at all. Slightly above this critical radius, heterogeneities of sufficient strength exert a very strong pinning force, outweighing any destabilizing effect (cf. figure $3.19 \mathrm{~b})$ ), quickly followed by the originally expected increase of $D_{\mathrm{L}}$.

The above results show two important facts: firstly, the basic mechanism of spiral wave stabilization observed in section 3.1.5 also extends to spatio-temporally chaotic dynamics. Again, the spatial scale of the heterogeneity is a critical parameter determining whether it will stabilize or destabilize the dynamics. Secondly and more fundamentally, heterogeneities can reduce the complexity of activation patterns even below values found for any of the involved parameters in a homogeneous setting by introducing additional spatial structure. A prediction of the behavior of the heterogeneous system based on information obtained for the homogeneous case is therefore difficult if not impossible.

\subsubsection{Brief summary}

In section 3.1, wave patterns in excitable media were characterized with the help of Lyapunov stability analysis. In sections 3.1.1, 3.1.2 and 3.1.3, spectra of different periodic attractors, corresponding to the well-known wave phenomena of plane waves, spiral waves, and multiple spiral waves, respectively, were examined in the Fenton-Karma model and the Barkley model. Fundamental properties of the leading Lyapunov spectra could be explained with the diffusive interaction between nearby points (plane waves), symmetries of the system (spiral waves) or the decoupling of different regions due to localized periodic wave sources and wave annihilation (multiple spiral waves). In all cases, the leading Lyapunov spectrum was found to be discrete, indicating finite-dimensional attractors despite the originally infinite-dimensional PDE. Although only a discretized system was simulated, continuous leading Lyapunov spectra could be ruled out due to the coupledoscillator analogy for plane waves and the convergence of the Lyapunov sepctrum with increasing resolution for spiral waves. In section 3.1.4, it was shown that bifurcation analysis of the meandering instability of spiral waves is possible in these high-dimensional systems using the Lyapunov spectrum. The results (including the bifurcating directions indicated by Lyapunov vectors) were found to be consistent with results of a linear stability analysis of the corresponding fixed point problem [107]. This information was used in sections 3.1 .5 and 3.1 .6 to investigate the influence of circular and random heterogeneities of lower excitability on spiral wave dynamics. Depending on the size and strength of 
the heterogeneities, opposing effects of stabilization and destabilization of the spiral wave could be observed, even leading to a heterogeneity-induced meandering bifuraction for discrete heterogeneities. However, the pinning effect of the heterogeneity always led to a stabilization of the symmetry modes, which could be identified from the spatial structure of Lyapunov vectors. Finally, in section 3.1.7, it was shown in the Bär-Eiswirth model that the stabilizing effects of less excitable regions also extend to the state of spatio-temporal chaos. Using Lyapunov stability analysis, this reduction in complexity (compared to the homogeneous case) could even be detected before an actual pinning effect in the spiral wave dynamics became visible. For a detailed summary and a discussion of the results, see section 4 . 


\subsection{Sensitivity of curved tissue boundaries to electric-field stimulation ${ }^{6}$}

In the introduction, it was suggested that the geometry of the cardiac muscle contributes significantly to its complexity. Several theoretical studies by A. PUMIR and S. TAKAgI et al. have shown that the internal tissue boundaries arising at the interface to electrically insulated circular regions of tissue can lead to size-dependent wave emission in response to relatively weak electric-field stimulation [127, 219, 128, 130]. In these studies, it was conjectured that inducing waves at heterogeneities in this way might be a superior way of unpinning pinned spiral waves. This conjecture was later confirmed in a simple generic model by comparison with anti-tachycardia pacing [131, 220], which suggests a possible relevance of wave induction at these tissue boundaries for antiarrhythmic therapies. Indeed, in references [135, 201], low-energy defibrillation was experimentally achieved in canine hearts based on weak electric field pulses and the success of the method could be related to wave source recruitment at the internal tissue boundaries originating from blood vessels. Although these experimental results shall be presented in detail in section 3.3 , they may serve here already as a strong indication for the importance of tissue boundaries in low-energy defibrillation - in contrast to other heterogeneities which were investigated in the context of single-shock, high-energy defibrillation (see section 1.7).

The aforementioned investigations by A. PUMIR and S. TAKAGI et al. predict that electric fields create zones of depolarization and hyperpolarization next to circular regions of insulated cardiac tissue. For a given electric field strength, the magnitude of the depolarization was shown to increase with increasing radius $R$ of the obstacle, converging to a characteristic value for $R \rightarrow \infty$ (i.e. a straight boundary) [127, 130]. Conversely, a smaller electric field strength is needed to bring the membrane potential of larger obstacles above the excitation threshold and induce an activation. According to this current state of knowledge, there should be a field strength below which no waves at all can be induced, due to the limiting value for the depolarization for large obstacles $R \rightarrow \infty$. However, the restriction of the above studies to circular shapes raises the question, whether and at what field strengths other kinds of tissue boundaries might serve as wave emitting sites, possibly allowing for the termination of certain arrhythmias at even lower field strengths. This question will be answered below by generalizing the theory to different prototypical shapes, revealing the basic mechanisms governing the depolarization induced at arbitrary tissue boundaries.

\subsubsection{Theoretical framework}

The theoretical and numerical investigations are based on the equivalent mono-domain approximation derived in section 2.1 .3 with the large-conductivity boundary condition of equation (2.16). For the following analysis, isotropic tissue is considered, which results in a scalar diffusion coefficient $D$ and conductivities $\sigma_{i}, \sigma_{e}$ and $\sigma_{o}$. Assuming a homogeneous electric field $\mathbf{E}$ outside the tissue, the outer potential is $v_{o}=-\mathbf{E} \cdot \mathbf{x}$. Substituting the outer potential into equation (2.16) leads to the following equations governing the dynamics of

\footnotetext{
${ }^{6}$ Meanwhile, some of the results of this section have been published in reference 218. Any display items identical with those in the publication are marked appropriately. Parts of the text may also be found in the paper.
} 
the medium:

$$
\begin{aligned}
\frac{\partial V_{m}}{\partial t} & =D \nabla^{2} V_{m}-I_{\text {ion }}\left(V_{m}, \mathbf{h}\right) / C_{m} & & \text { in } \mathcal{D} \\
\frac{\mathrm{d} \mathbf{h}}{\mathrm{d} t} & =\mathbf{H}\left(V_{m}, \mathbf{h}\right) & & \\
\mathbf{n} \cdot \nabla V_{m} & =\mathbf{n} \cdot \frac{\sigma_{o}}{\sigma_{e}} \mathbf{E} & & \text { on } \partial \mathcal{D} .
\end{aligned}
$$

Equations (3.13a) and 3.13b can be combined to one system of partial differential equations for the state vector $\mathbf{u}=\left(V_{m}, \mathbf{h}\right)$ :

$$
\frac{\partial \mathbf{u}}{\partial t}=\mathbf{f}(\mathbf{u})+\mathbf{e}_{1} D \nabla^{2} u_{1}
$$

where $\mathbf{f}=\left(-I_{\text {ion }} / C_{m}, \mathbf{H}\right)$ and $\mathbf{e}_{1}=(1,0,0, \ldots)$ is the first basis vector which limits diffusion to the membrane potential $u_{1}=V_{m}$.

Equation (3.14) cannot be solved analytically (neither with nor without the modified boundary condition). In order to still derive some quantitative measure for the effect of $\mathbf{E}$, equation 3.14 is linearized around the resting state of the membrane potential and the gating variables $\mathbf{u}_{0}=\left(V_{m}^{0}, \mathbf{h}^{0}\right)$ :

$$
\frac{\partial \delta \mathbf{u}}{\partial t}=\left.\mathrm{J}_{\mathbf{f}}\right|_{\mathbf{u}_{0}} \delta \mathbf{u}+\mathbf{e}_{1} D \nabla^{2} \delta u_{1}
$$

$\mathrm{J}_{\mathbf{f}}$ is the Jacobian matrix of the function $\mathbf{f}$. If the function $\mathbf{H}$ describing the ion channel dynamics is independent of $V_{m}$ below some finite threshold $V_{m}^{\text {thresh }}>V_{m}^{0}$, then $\delta u_{1}$ is independent of all $\delta u_{i}, i>1$, and the latter have a fixed value. In this case, the linearization corresponds to the passive dynamics of the membrane [122, 123, 125]. The steady state of equation 3.15, obtained by setting the left-hand side to zero, yields the membrane potential distribution and gating variables that represent the response to an infinitesimal electric field ${ }^{7}$ applied for infinite time. Only the first row of the vector equation contains a diffusion term, making it possible to express $\delta u_{2}, \delta u_{3}, \ldots$ in the first row as a function of $\delta u_{1}$ (assuming that $\mathrm{J}_{\mathbf{f}}$ with the first row and column removed is invertible). As $\delta u_{1}$ is actually the deviation of the membrane potential, let $e:=\delta u_{1}$, such that the following time-independent equations for $e$ are obtained:

$$
\begin{aligned}
\nabla^{2} e-\frac{e}{\lambda^{2}} & =0 & & \text { on } \mathcal{D} \\
\mathbf{n} \cdot(\nabla e-\mathbf{E}) & =0 & & \text { on } \partial \mathcal{D}
\end{aligned}
$$

$\lambda$ is the electrotonic constant, resulting from the replacement procedure of the $\delta u_{i}, i>1$. The second equation is the boundary condition of equation 3.13c), with the conductivity ratio absorbed into E. It should be emphasized that the modified boundary condition implementing the approximate bi-domain effect of an electric field is the only place in equation (3.16) as well as in the full model of equation (3.13) which depends on the electric field. Thus, within this approximation, the application of an electric field can be included in any simulation simply by imposing the modified boundary condition. This fact

\footnotetext{
${ }^{7}$ Infinitesimal deviation $\delta \mathbf{u}$ from the resting state and infinitesimal electric fields are equivalent assumptions, as the resting state is known to be stable.
} 
will be exploited towards the end of this investigation, when finite field strengths outside the scope of the linear approximation 3.16 are considered. In the following, equations $3.16 \mathrm{a}$ and $3.16 \mathrm{~b}$ will be solved on domains $\mathcal{D}$ of different prototypical shapes 8

\subsubsection{Setup of numerical simulations}

For domains $\mathcal{D}$ that do not allow an analytical solution of equation (3.16) and to check the validity of the analytical calculations presented in the following sections, direct numerical simulations with the Fenton-Karma model (see section 2.1.6) are carried out using parameter set FK1 (see table A.2, page 177). The modified boundary condition 3.16b is imposed via the phase-field method as outlined in sections 2.1.5 and 2.2.3, using a very small field strength of $|\mathbf{E}|=1 \mu \mathrm{V} / \mathrm{cm}$. The depolarization pattern is allowed to equilibrate for a time span of $1000 \mathrm{~ms}$, before the maximum depolarization is determined. For the computation of the phase fields, $\xi^{2}=0.72 h^{2}$ is used in equation 2.21, where $h$ is the space step of the simulation. For increased accuracy of the resulting phase field $\phi, \phi_{0}$ is not binary, but the value of each numerical pixel is equal to the fractional overlap of the pixel with the domain $\mathcal{D}$. This is possible here, because $\mathcal{D}$ will be given analytically. The default space step is $h=100 \mu \mathrm{m}$, but for certain problems involving small geometrical features, a smaller $h$ is used. The time step is $\Delta t=0.1 \cdot h^{2} / D$ throughout, in order to fulfill the stability criterion of equation (2.93). This corresponds to $\Delta t=10 \mu \mathrm{s}$ for the default value of $h$.

For the Fenton-Karma model, the state vector $\mathbf{u}$ from equation 3.14 is given by $\mathbf{u}=$ $(u, v, w)$. The resting state is $\mathbf{u}_{0}=\left(u_{0}, v_{0}, w_{0}\right) \approx(0,1,1)$ (see section 2.1.6, page 45). Under the assumption that $u_{c}, u_{v}>0$, the Jacobian of equation (3.15) evaluates to

$$
\left.\mathrm{J}_{f}\right|_{\mathbf{u}_{0}}=\left(\begin{array}{ccc}
\delta & 0 & \epsilon \\
0 & -1 / \tau_{v 2}^{-} & 0 \\
0 & 0 & -1 / \tau_{w}^{-}
\end{array}\right),
$$

where $\delta=-\frac{1}{\tau_{0}}+\frac{w_{0} k}{2 \tau_{s i}}\left(1-\tanh ^{2}\left[k\left(u_{0}-u_{c}^{s i}\right)\right]\right)$ and $\epsilon=\frac{1}{2 * \tau_{s i}}\left(1+\tanh \left[k \cdot\left(u_{0}-u_{c}^{s i}\right)\right]\right)$ (cf. the full Jacobian in appendix A). Substituting this Jacobian into equation 3.15 and setting the left-hand side to zero yields

$$
\lambda=\sqrt{-D / \delta}
$$

in equation 3.16a). If $u_{c}^{s i}$ is large enough, such that $\tanh \left[k \cdot\left(u_{0}-u_{c}^{s i}\right)\right] \approx-1$ (which is the case for the parameters used here), then $\delta$ reduces to $-\frac{1}{\tau_{0}}$, which means that $\lambda \approx \sqrt{D \tau_{0}}$. For the FK1 parameters, this expression leads to $\lambda=1.12 \mathrm{~mm} 9$

\footnotetext{
${ }^{8}$ Equation (3.16) is identical to the ad-hoc ansatz used in references [127, 130, to assess electric-field induced membrane potential changes for circular obstacles. However, those publications use a different sign in equation $3.16 \mathrm{~b}$. Private communication with the main author, AlAIN PUMIR, led to the finding that the latter is a misprint.

${ }^{9}$ The size and curvature of the tissue domains can be normalized by $\lambda$, as explained in section 3.2 .3 . Therefore, the actual parameters used for the Fenton-Karma model do not alter the results. The only condition is that the field strength used is small enough to preserve the validity of equation 3.16 .
} 


\subsubsection{Generic properties of induced membrane potential changes}

Some properties of the membrane potential distribution $e$ can be directly deduced from equation (3.16). By defining a normalized coordinate system $\mathbf{x}^{*}=\mathbf{x} / \lambda$ (with a corresponding gradient $\nabla^{*}$ and transformed tissue domains) and the normalized membrane potential deviation as $e^{*}=e /(\lambda|\mathbf{E}|)$, equation (3.16) can be rewritten as

$$
\begin{aligned}
\nabla^{* 2} e^{*}-e^{*} & =0 & & \text { on } \mathcal{D}^{*} \\
\mathbf{n} \cdot\left(\nabla^{*} e^{*}-\hat{\mathbf{E}}\right) & =0 & & \text { on } \partial \mathcal{D}^{*},
\end{aligned}
$$

where $\hat{\mathbf{E}}=\mathbf{E} /|\mathbf{E}|$ is the unit vector in the direction of the electric field $\sqrt{10}$. Two different problems with tissue domains $\mathcal{D}_{1}$ and $\mathcal{D}_{2}$ and respective electrotonic constants $\lambda_{1}$ and $\lambda_{2}$ thus have the same solution $e^{*}$, if $\mathcal{D}_{1}$ can be transformed to $\mathcal{D}_{2}$ by a scaling transformation: $\mathcal{D}_{1}=\frac{\lambda_{1}}{\lambda_{2}} \mathcal{D}_{2}$. The electric field strength $|\mathbf{E}|$ does not appear in equation 3.19$)$ and therefore is completely irrelevant for the solution $e^{*}$. From the definition $e^{*}$ it is evident that the solution $e$ for the original problem is simply proportional to $|\mathbf{E}|$.

In contrast, the direction $\hat{\mathbf{E}}$ of the electric field is generally important: The larger the component of $\hat{\mathbf{E}}$ along $\mathbf{n}$, i.e. perpendicular to the boundary, the larger the deviation of equation $3.19 \mathrm{~b}$ from the usual homogenous Neumann (no-flux) boundary condition. Large effects can therefore be expected at boundaries perpendicular to the direction of the electric field. The sign of the induced membrane potential deviation can be heuristically determined from equation (3.16): The effect of the electric field should vanish far away from the boundary. Assuming a monotonic decay of $e$ towards zero in the direction away from the boundary of the tissue due to the diffusion term, $e>0$ at the boundary implies $\frac{\partial e}{\partial n}<0$ (with $\mathbf{n}$ pointing into the tissue). On the other hand, according to equation $3.16 \mathrm{~b}, \frac{\partial e}{\partial n}=\mathbf{n} \cdot \mathbf{E}$, which means that $\mathbf{E}$ must have a component anti-parallel to $\mathbf{n}$ and is therefore pointing in the half-space out of the tissue. Consequently, depolarizations and hyperpolarizations of the tissue are expected to occur on parts of the boundary with the electric field vector pointing out of and into the tissue, respectively.

\subsubsection{Tissue domains of different dimension}

Let $\mathcal{D}^{2 \mathrm{~d}} \subset \mathbb{R}$ be an arbitrary two-dimensional tissue domain and $\mathcal{D}^{3 \mathrm{~d}}=\{(x, y, z) \in$ $\left.\mathbb{R}^{3} \mid(x, y) \in \mathcal{D}^{2 \mathrm{~d}} \wedge z \in I\right\}$ its extension to three dimensions, where $I \subset \mathbb{R}$ is either an interval $I=[a, b]$ or the whole of $\mathbb{R}$. Let $e^{2 \mathrm{~d}}(x, y)$ be a solution to equation 3.16 for $\mathcal{D}^{2 \mathrm{~d}}$ with an electric field $\mathbf{E}^{2 \mathrm{~d}}$. Define the extensions to three dimensions as

$$
\begin{aligned}
e^{3 \mathrm{~d}}(x, y, z) & :=e^{2 \mathrm{~d}}(x, y) \quad \text { on } \mathcal{D}^{3 \mathrm{~d}} \\
\mathbf{E}^{3 \mathrm{~d}} & :=\left(E_{x}^{2 \mathrm{~d}}, E_{y}^{2 \mathrm{~d}}, 0\right) .
\end{aligned}
$$

For $I=\mathbb{R}$, it follows that $\partial \mathcal{D}^{3 \mathrm{~d}}=\left\{(x, y, z) \in \mathbb{R}^{3} \mid(x, y) \in \partial \mathcal{D}^{2 \mathrm{~d}}\right\}$. This means that everywhere on the boundary the normal vector is just

$$
\mathbf{n}^{3 \mathrm{~d}}(x, y, z)=\left(n_{x}^{2 \mathrm{~d}}(x, y), n_{y}^{2 \mathrm{~d}}(x, y), 0\right) \quad \text { on } \partial \mathcal{D}^{3 \mathrm{~d}} .
$$

\footnotetext{
${ }^{10}$ As $\mathbf{E}$ has units of a potential divided by a length, applying the same transformations as for $\mathbf{x}$ and $e$ automatically leads to a unit $\mathbf{E}^{*}$, thus being identical to $\hat{\mathbf{E}}$ defined above.
} 
Substituting equations $3.20,3.21$ and 3.22 into equation 3.16 for $\mathcal{D}^{3 \mathrm{~d}}$ leads to

$$
\begin{aligned}
& \nabla^{2} e^{3 \mathrm{~d}}-\frac{e^{3 \mathrm{~d}}}{\lambda^{2}} \quad=\frac{\partial^{2} e^{2 \mathrm{~d}}}{\partial x^{2}}+\frac{\partial^{2} e^{2 \mathrm{~d}}}{\partial y^{2}}+\overbrace{\frac{\partial^{2} e^{2 \mathrm{~d}}}{\partial z^{2}}}^{=0}-\frac{e^{2 \mathrm{~d}}}{\lambda^{2}} \quad 2.16) \stackrel{\text { for }}{=} \mathcal{D}^{2 \mathrm{~d}} 0 \quad \text { on } \mathcal{D}^{3 \mathrm{~d}}
\end{aligned}
$$

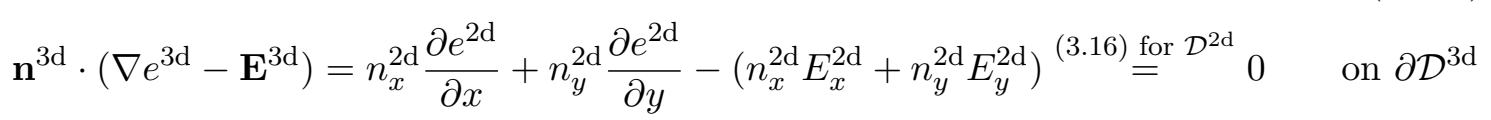

which implies that $e^{3 \mathrm{~d}}$ as defined in equation 3.20 is a solution of the three-dimensional problem.

In the case of $I=[a, b], \partial \mathcal{D}^{3 \mathrm{~d}}$ has two additional boundaries parallel to the $z$-plane at $z=a$ and $z=b$, i.e.

$$
\begin{aligned}
\partial \mathcal{D}^{3 \mathrm{~d}}= & \underbrace{\left\{(x, y, z) \in \mathbb{R}^{3} \mid(x, y) \in \partial \mathcal{D}^{2 \mathrm{~d}} \wedge z \in I\right\}}_{=: \partial \mathcal{D}_{1}^{3 \mathrm{~d}}} \\
& \cup \underbrace{\left\{(x, y, z) \in \mathbb{R}^{3} \mid(x, y) \in \mathcal{D}^{2 \mathrm{~d}} \wedge(z=a \vee z=b)\right\}}_{=: \partial \mathcal{D}_{2}^{3 \mathrm{~d}}} .
\end{aligned}
$$

Equation 3.22 remains valid for $\partial \mathcal{D}_{1}^{3 \mathrm{~d}}$, but for the new parts of the boundary, instead

$$
\mathbf{n}^{3 \mathrm{~d}}(x, y, z)=(0,0,1) \quad \text { on } \partial \mathcal{D}_{2}^{3 \mathrm{~d}}
$$

holds. Because of the definition of $e^{3 \mathrm{~d}}$, equation $3.23 \mathrm{a}$ remains valid and equation $3.23 \mathrm{~b}$ does still hold for $\partial \mathcal{D}_{1}^{3 \mathrm{~d}}$ (because it is a subset of $\partial \mathcal{D}^{3 \mathrm{~d}}$ considered in equation $(3.23 \mathrm{~b})$ ). For the additional boundary points the boundary condition of equation $3.16 \mathrm{~b})$ is

$$
\mathbf{n}^{3 \mathrm{~d}} \cdot\left(\nabla e^{3 \mathrm{~d}}-\mathbf{E}^{3 \mathrm{~d}}\right)=1 \cdot \overbrace{\frac{\partial e^{2 \mathrm{~d}}}{\partial z}}^{=0}-1 \cdot \overbrace{E_{z}^{3 \mathrm{~d}}}^{=0}=0 \quad \text { on } \partial \mathcal{D}_{2}^{3 \mathrm{~d}},
$$

which means that the boundary condition is still fulfilled on the whole of $\partial \mathcal{D}^{3 \mathrm{~d}}$.

In summary, the above argument shows that, for an unskewed " $z$-stack" of identical twodimensional shapes, the solution to equation (3.16) looks like the corresponding solution for the two-dimensional shape in every cross-section parallel to the $z$-plane with $z \in I$, if the electric field vector lies also in the $z$-plane ${ }^{11}$. For example, for a (finite or infinite) cylinder, the depolarization pattern in every cross-section perpendicular to the cylinder axis is identical to that of the corresponding disk in two dimensions.

The same line of thoughts also applies to a one-dimensional $\mathcal{D}^{1 \mathrm{~d}}$ and its extension $\mathcal{D}^{2 \mathrm{~d}}=$ $\left\{(x, y) \in \mathbb{R}^{2} \mid x \in \mathcal{D}^{1 \mathrm{~d}} \wedge y \in I\right\}$, again with $I=\mathbb{R}$ or $I=[a, b]$. The solution for the rectangle $\mathcal{D}^{2 \mathrm{~d}}=[\alpha, \beta] \times[a, b] \subset \mathbb{R}^{2}$ with an electric field parallel to the $x$-axis therefore looks, for every fixed $y \in[a, b]$, like the one-dimensional solution on the interval $[\alpha, \beta]$.

\footnotetext{
${ }^{11}$ In fact, for the infinite stack, i.e. $I=\mathbb{R}$, the electric field can also have a $z$-component, which does, however, not change the solution, because all normal vectors have vanishing $z$-components according to equation 3.22
} 


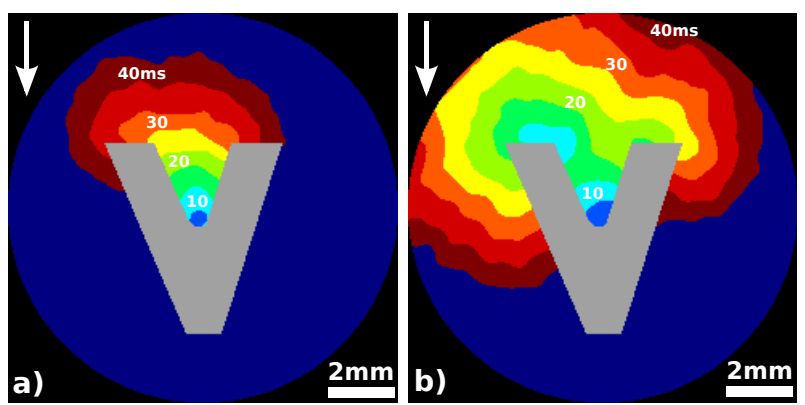

Figure 3.22: Boundary effects in two-dimensional cardiac cell culture experiments. Cultured neonatal rat cardiac cells were stimulated with vertical electric fields (white arrows) of a) $0.91 \mathrm{~V} / \mathrm{cm}$ and $\mathbf{b}) 1.37 \mathrm{~V} / \mathrm{cm}$ and activity was imaged using the fluorescent $\mathrm{Ca}^{2+}$ indicator Fluo-8. Colors indicate time from pacing to activation, gray color marks regions without tissue. At higher field strengths, the flat parts of the boundary are excited as well as the tip of the $\mathrm{V}$-shaped boundary. For the lower electric field strength, excitation can only be induced at the latter location. A series of $N=9$ experiments yielded a field strength ratio of $1.49 \pm 0.26$ between the two types of boundaries. Reproduced from [218].

\subsubsection{Curvature dependence in cell culture experiments ${ }^{12}$}

A strong indication for the relevance of the geometric shape of the outer tissue boundaries can be obtained in experiments on cardiac myocyte cultures with artificial boundaries, but an otherwise homogeneous cell monolayer. Activation maps of such cell cultures for electric-field stimulation with two different field strengths are shown in figure 3.22, During the culturing process on a cover slip, a region was blocked with a V-shaped obstacle manufactured out of polydimethylsiloxane (PDMS) to prevent cardiac cells from attaching. The obstacle itself was removed during the culturing process, but a V-shaped region containing no cells was left behind, which is marked gray in figure 3.22 . For a detailed description of the experimental method, see the Supplementary Material of reference [218]. Figure 3.22 b) demonstrates that the membrane potential changes induced by electric fields can be used for exciting the tissue at the boundary. Moreover, the predictions of section 3.2 .3 are verified in that the tissue is excited at those boundaries, where the electric field vector points out of the tissue (upper edge of the $\mathrm{V}$-shaped gray region), and in that boundaries perpendicular to the electric field are the most relevant ones. However, stimulation with a lower field strength shown in figure 3.22 ) reveals that the field strength necessary to induce excitation is significantly different for tissue boundaries of convex and flat shape. This behavior was found to be consistent across $N=9$ different cell cultures prepared in the same way: On average, the minimum field strength necessary to elicit an excitation at the flat boundary was $1.49 \pm 0.26$ times as large as the field strength required to excite the convex tip of the boundary. The individual threshold field strengths for each cell culture are plotted against each other in figure 3.23 . Specific geometrical features thus enable the recruitment of additional localized wave emitting sites in the low field-strength range - an effect that has not been studied before.

\footnotetext{
${ }^{12}$ The experiments presented in this section have been carried out and the analysis was performed by MARCEL HörNing, who is a co-author of reference 218.
} 


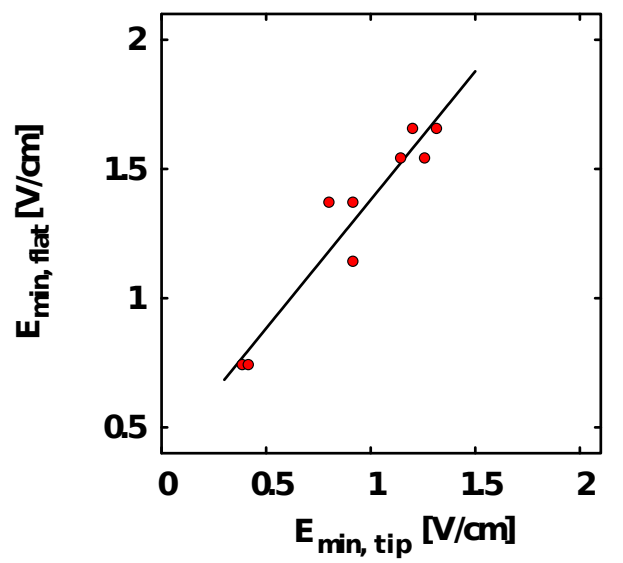

Figure 3.23: Increased susceptibility of convex boundaries. Minimum field strength necessary to induce excitation at the flat parts of the V-shaped boundary (cf. figure 3.22) plotted against the corresponding field strength for the curved tip of the boundary. The absolute field strength necessary varies across different samples. However, the experiments yield a reproducible difference between the two types of boundaries, as indicated by the linear fit.

\subsubsection{Definition of boundary curvature}

Motivated by the experimental evidence shown in section 3.2.5, in the following, different boundaries $\partial \mathcal{D}$ of one- to three-dimensional tissue domains $\mathcal{D}$ will be characterized by their curvature at the point of maximum depolarization. The definition of curvature that will be used throughout is the mean curvature $\kappa=\left(\kappa_{1}+\kappa_{2}\right) / 2$, where $\kappa_{1}$ and $\kappa_{2}$ are the two principal curvatures of the surface $\partial \mathcal{D}$, given by the two curves through the point under consideration with maximum and minimum curvature.

Following from the dimensionality argument in section 3.2.4, an additional dimension of the boundary with curvature zero does not change the solution $e$ of equation (3.16). Thus, in order to have a curvature measure consistent across different dimensions, $\kappa_{2}=0$ is assumed for two-dimensional $\mathcal{D}$, i.e. one-dimensionl $\partial \mathcal{D}$. For zero-dimensional, point-like $\partial \mathcal{D}$ originating from the ends of a one-dimensional tissue domain $\mathcal{D}$ principal curvatures $\kappa_{1}=\kappa_{2}=0$ are assumed, corresponding to flat boundaries in higher dimensions.

As the sign of the curvature depends on the direction of the normal vector (either into or out of the domain), in the context of this study, boundaries of convex domains are defined to have negative curvature: the boundary of a circular domain of radius $R$ thus has a curvature of $\kappa_{1}=-1 / R$, corresponding to a mean curvature of $\kappa=-1 /(2 R)$. In contrast, a hole of radius $R$ in an otherwise infinite domain has mean curvature $\kappa=1 /(2 R)$.

As explained in section 3.2.3, dimensionless space units can be obtained by a normalization with the electrotonic constant $\lambda$. As the curvature $\kappa$ has units [1/length], the normalized curvature that will be used throughout this section is $\kappa^{*}=\kappa \lambda$.

\subsubsection{Flat boundaries}

Applying the results of section 3.2.4 the induced membrane potential change predicted by equation (3.16) next to a flat boundary in two or three dimensions can be calculated by considering a one dimensional tissue domain $\mathcal{D}=[a, b] \subset \mathbb{R}$, where either $a=-b$ (for a bounded domain), or $a=-\infty, b=0$ (for an unbounded domain). Given an electric field 


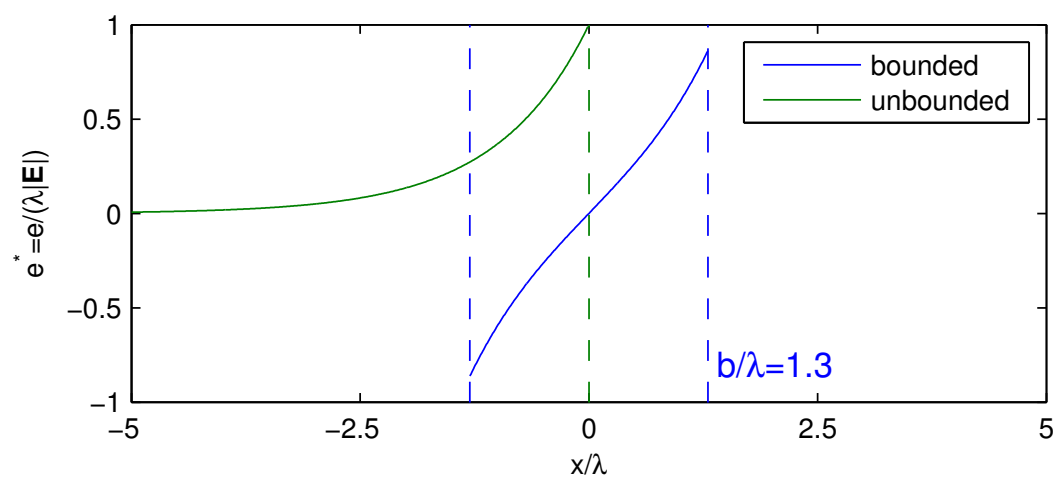

Figure 3.24: Depolarization in a one-dimensional tissue domain. The solutions of equations $(3.27)(b=1.3 \lambda)$ and $(3.28)$ are plotted as a blue and green line, respectively. Vertical dashed lines of the same color indicate tissue boundaries.

strength $\mathbf{E}$ in positive $x$-direction, which is just a positive scalar quantity in this case, the solutions obtained by elementary calculus are

$$
\begin{aligned}
e_{\text {bounded }}(x) & =\lambda|\mathbf{E}| \frac{\sinh (x / \lambda)}{\cosh (b / \lambda)} & \text { on }[-b, b] \\
e_{\text {unbounded }}(x) & =\lambda|\mathbf{E}| \exp (x / \lambda) & \text { on }[-\infty, 0] .
\end{aligned}
$$

For the unbounded domain, it is assumed that the effect of the electric field vanishes for $x \rightarrow-\infty$. Both solutions (see figure 3.24) are monotonically increasing, so the maximum depolarization occurs at the right end of the domain in both cases: $e_{\text {bounded }}^{\max }=e_{\text {bounded }}(b)=$ $\lambda|\mathbf{E}| \tanh (b / \lambda)$ and $e_{\mathrm{unbounded}}^{\max }=e_{\text {unbounded }}(0)=\lambda|\mathbf{E}|$. Consistently, $e_{\mathrm{bounded}}^{\max }$ approaches $e_{\text {unbounded }}^{\max }$ for $b \rightarrow \infty$.

For the bounded case, the electric field causes a hyperpolarization at the left side of the domain, which is equal in magnitude to the depolarization on the right side. The maximum depolarization decreases with decreasing domain size. For $b / \lambda \ll 1$ it asymptotically behaves like $e_{\text {bounded }}^{\max } \approx|\mathbf{E}| b$. Inside the domain, the argument of the sinh in equation (3.27) is even smaller, such that the $x$-dependence for domains that are very small compared to $\lambda$ simplifies to $e_{\text {bounded }}(x) \approx|\mathbf{E}| x$, and thus becomes linear ${ }^{13}$. Physically, the decrease of the maximum depolarization can be interpreted as a merging of the two effects from the left and right boundary: because of the diffusive nature of the system, hyperpolarization and depolarization more and more cancel each other, depending on the distance between the two boundaries. This is because the characteristic length for the diffusion (the electrotonic constant $\lambda$ ) is fixed. This kind of source-sink interaction was also confirmed in a study on cable-shaped cell cultures by A. KONDRATYEV [221].

\subsubsection{Circular boundaries}

Circular boundaries arising from circular inexcitable regions of cardiac tissue (cf. figure 3.25 ) ) are analytically tractable, yet important, geometrical shapes, as they represent cross sections of blood vessels in heart, as indicated in the beginning of this section. The

\footnotetext{
${ }^{13}$ As very small domains are equivalent to very large $\lambda$ (cf. section 3.2 .4 ), this is also clear directly from equation 3.16a), which turns into a homogeneous Laplace equation for large $\lambda$.
} 
a)

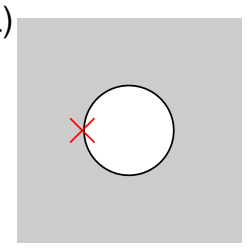

b)

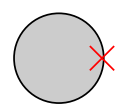

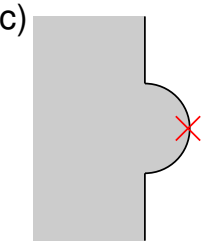

d)

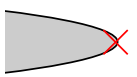

Figure 3.25: Two dimensional shape prototypes. Different kinds of shapes examined for their interaction with electric field stimulation. Positions in gray regions are $\in \mathcal{D}$, black lines indicate $\partial \mathcal{D}$. " $\times$ " indicate points, where the maximimum depolarization is expected for an electric field pointing to the right following the argumentation in section 3.2 .3 .

inverse (convex) case, depicted in figure $3.25 \mathrm{~b}$ ), which has not been considered before, can be thought of as a cross section through, e.g., papillary muscles or trabeculae in places where they are not directly attached to the endocardial wall (see sections 1.1 and 1.3 . Let $e_{a}$ and $e_{b}$ denote the solutions for the two shapes of figure $3.25 \mathrm{a}$ ) and figure $3.25 \mathrm{~b}$ ), respectively. For both cases, the boundary condition of equation $3.19 \mathrm{~b}$ takes the same form

$$
\left.\frac{\partial e_{a, b}^{*}}{\partial r^{*}}\right|_{r^{*}=R^{*}}=\cos (\varphi) \quad \forall \varphi \in[-\pi, \pi[,
$$

if an electric field in positive $x$-direction is assumed and the solution is written in polar coordinates $\left(r^{*}, \varphi\right)$ ( "*" denotes the normalized coordinates introduced in section 3.2.3). $R^{*}=R / \lambda$ is the radius of the circle in normalized coordinates. Substituting the ansatz $e_{a, b}^{*}=c g\left(r^{*}\right) \cos (\varphi)$ into equation 3.19 a yields

$$
\begin{aligned}
{\left[\frac{\partial^{2}}{\partial r^{* 2}}+\frac{1}{r^{*}} \frac{\partial}{\partial r^{*}}+\frac{1}{r^{* 2}} \frac{\partial^{2}}{\partial \varphi^{2}}\right]\left(g\left(r^{*}\right) \cos (\varphi)\right) } & & =g\left(r^{*}\right) \cos (\varphi) \\
\Rightarrow \quad g^{\prime \prime}\left(r^{*}\right) \cos (\varphi)+\frac{1}{r^{*}} g^{\prime}\left(r^{*}\right) \cos (\varphi)-\frac{1}{r^{* 2}} g\left(r^{*}\right) \cos (\varphi) & & =g\left(r^{*}\right) \cos (\varphi) \\
\Rightarrow \quad r^{* 2} g^{\prime \prime}\left(r^{*}\right)+r^{*} g^{\prime}\left(r^{*}\right)-\left(1+r^{* 2}\right) g\left(r^{*}\right) & & =0 .
\end{aligned}
$$

Equation 3.30 is the differential equation for the modified Bessel function of order 1, which has two linearly independent solutions $I_{1}$ and $K_{1}$, namely the modified Bessel functions of the first and second kind, respectively. $I_{1}$ diverges for $r^{*} \rightarrow \infty$, whereas $K_{1}$ diverges for $r^{*} \rightarrow 0$. The effect of the electric field is expected to vanish at infinite distance from the obstacle in case of figure $3.25 \mathrm{a}$ ) and is expected to stay finite in all of $\mathcal{D}$ for the shape of figure $3.25 \mathrm{~b}$ ). Therefore, for the solution $e_{a}^{*}$, only $K_{1}$ comes into consideration, and likewise only $I_{1}$ for $e_{b}^{*}$. 
The factor $c$ in the ansatz is determined from equation 3.29 :

$$
\begin{aligned}
\left.\frac{\partial e_{a, b}^{*}}{\partial r^{*}}\right|_{r^{*}=R^{*}}=c g^{\prime}\left(R^{*}\right) \cos (\varphi) & =\cos (\varphi) \\
\Rightarrow \quad c & =\frac{1}{g^{\prime}\left(R^{*}\right)}
\end{aligned}
$$

The solutions for the boundary condition of equation 3.29 therefore read

$$
\begin{array}{ll}
e_{a}=\lambda|\mathbf{E}| e_{a}^{*}=\lambda|\mathbf{E}| \frac{K_{1}(r / \lambda)}{K_{1}^{\prime}(R / \lambda)} \cos (\varphi) & \text { defined for } r>R \\
e_{b}=\lambda|\mathbf{E}| e_{b}^{*}=\lambda|\mathbf{E}| \frac{I_{1}(r / \lambda)}{I_{1}^{\prime}(R / \lambda)} \cos (\varphi) & \text { defined for } r<R
\end{array}
$$

The first one is identical to the solution obtained in reference [127] and has its maximum at $(r, \varphi)=(R, \pi)$, because $K_{1}$ is monotonically decreasing and the solution is only defined for $r>R$. As $I_{1}$ is monotonically increasing, correspondingly, the solution $e_{b}$ for circular $\mathcal{D}$ has its maximum at $(r, \varphi)=(R, 0)$. As a circular boundary of radius $R$ has equal curvature $1 / R$ everywhere, the mean curvatures at the point of maximum depolarization are $\kappa_{a}=1 /(2 R)$ and $\kappa_{b}=-1 /(2 R)$, using the definition introduced in section 3.2.6. The maximum depolarizations are therefore

$$
\begin{aligned}
& e_{a}^{\max }=-\lambda|\mathbf{E}| \frac{K_{1}(R / \lambda)}{K_{1}^{\prime}(R / \lambda)}=-\lambda|\mathbf{E}| \frac{K_{1}\left(1 /\left(2 \kappa_{a} \lambda\right)\right)}{K_{1}^{\prime}\left(1 /\left(2 \kappa_{a} \lambda\right)\right)} \\
& e_{b}^{\max }=\lambda|\mathbf{E}| \frac{I_{1}(R / \lambda)}{I_{1}^{\prime}(R / \lambda)}=\lambda|\mathbf{E}| \frac{I_{1}\left(-1 /\left(2 \kappa_{b} \lambda\right)\right)}{I_{1}^{\prime}\left(-1 /\left(2 \kappa_{b} \lambda\right)\right)} .
\end{aligned}
$$

Exemplary depolarization patterns corresponding to equations (3.33) and (3.34) are depicted in the bottom and top row of figure 3.26 , respectively. The last image of the top row and the first image of the bottom row of figure 3.26 show that both solutions (3.33) and (3.34) describe the same scenario for the depolarized boundary in the limiting case of $R \rightarrow \infty$. Indeed, assuming large $R$, the solutions near the depolarized boundary read

$$
\begin{aligned}
& e_{a}=\lambda|\mathbf{E}| \frac{K_{1}(\overbrace{r}^{\approx-x} / \lambda)}{K_{1}^{\prime}(R / \lambda)} \cos (\overbrace{\varphi}^{\approx \pi}) \approx \lambda|\mathbf{E}| \frac{\exp (x / \lambda)}{-\exp (-R / \lambda)} \cdot(-1) \\
& =\lambda|\mathbf{E}| \exp [(x+R) / \lambda] \text { for } x<-R \\
& e_{b}=\lambda|\mathbf{E}| \frac{I_{1}(\overbrace{r}^{\approx x} / \lambda)}{I_{1}^{\prime}(R / \lambda)} \cos (\overbrace{\varphi}^{\approx 0}) \approx \lambda|\mathbf{E}| \frac{\exp (x / \lambda)}{\exp (R / \lambda)} \cdot 1 \\
& =\lambda|\mathbf{E}| \exp [(x-R) / \lambda] \quad \text { for } x<R .
\end{aligned}
$$

\footnotetext{
${ }^{14}$ As indicated in section 2.1 .3 the assumption of a constant electric field vector determining the boundary condition of equation $3.16 \mathrm{~b}$ and ultimately the cos-term on the right hand side of equation 3.29 is only an approximation. In case a more realistic boundary condition is determined in the future and the right hand side of equation 3.29 is replaced with some arbitrary function $f(\varphi)$, the mathematical strategy as presented here could be applied by writing $f$ as a sum of its Fourier modes $a_{n} \cos (n \varphi), b_{n} \sin (n \varphi)$. With the ansatz $e_{a, b}^{*}=c g\left(r^{*}\right) \cos (n \varphi)$ (or similarly with $\sin$ ), following the derivation would lead to solutions involving $K_{n}$ and $I_{n}$, where $n=1$ is only the special case considered above. As the Neumann boundary condition of equation 3.29 is additive, the solution could then be written as a sum of these partial solutions.
} 

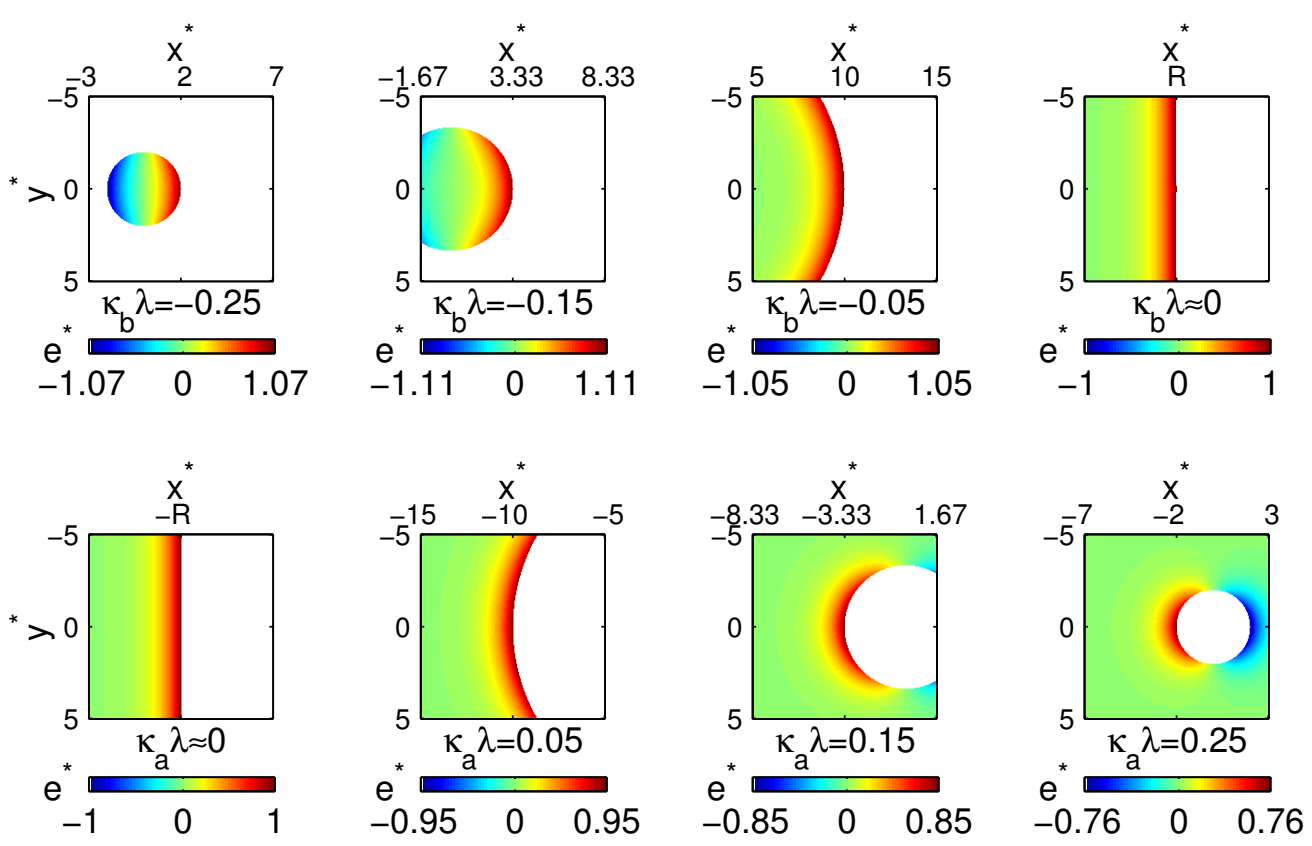

Figure 3.26: Membrane potential changes at circular boundaries. Each plot shows a depolarization pattern for a circular boundary of different positive and negative normalized mean curvature $\kappa \lambda$. The images are centered at the point of maximum depolarization (value indicated by colorbar limits). Length and depolarization units are normalized. White color indicates regions that are not part of the active tissue domain $\mathcal{D}$. Top row: negative curvature boundaries, solution according to equation (3.34). Bottom row: positive curvature boundaries, solution according to equation $(3.33)$.

In the above calculation, the identities $K_{n}^{\prime}=-\left(K_{n-1}+K_{n+1}\right) / 2$ and $I_{n}^{\prime}=\left(I_{n-1}+I_{n+1}\right) / 2$ were used (third line of equation 9.6.26 in [222], pp. 376). Furthermore, for large arguments $x$, the Bessel functions can be approximated by $K_{n}(x) \approx \sqrt{\pi /(2 x)} \exp (-x)$ and $I_{n}(x) \approx \sqrt{1 /(2 \pi x)} \exp (x)$ (truncated form of equations 9.7.1 and 9.7.2 in [222], pp. 377378 ). For large $R$ and near the points on the boundary which are maximally depolarized, both solutions (3.37) and (3.38) for circular boundaries thus become identical to the solution (3.28) for a flat boundary, which is located, however, at $x=-R$ and $x=R$, respectively. This fact indicates that, for large enough $R$, the existence of a hyperpolarized boundary on the opposite side of the circular shape can be neglected, which is reasonable due to the finite diffusive interaction length represented by the electrotonic space constant $\lambda$. Consequently, for $\kappa_{a}=0$ and $\kappa_{b}=0$, the maximum depolarization at the boundary is $e_{a, b}^{\max }=\lambda|\mathbf{E}|$. The same result can be obtained by taking the limit of either equation (3.35) or (3.36) for $\kappa_{a}$ and $\kappa_{b}$ towards zero.

The two cases considered above can therefore be assumed to originate from the same limiting case: a flat boundary, which is subsequently bent inwards or outwards and then continued with constant curvature. In normalized units, the maximum depolarization near a circular boundary of curvature $\kappa^{*}$ is therefore given by the function:

$$
e_{\text {circ }}^{* \max }\left(\kappa^{*}\right)= \begin{cases}-\frac{K_{1}\left(1 /\left(2 \kappa^{*}\right)\right)}{K_{1}^{\prime}\left(1 /\left(2 \kappa^{*}\right)\right)} & \kappa^{*}>0 \\ \frac{I_{1}\left(-1 /\left(2 \kappa^{*}\right)\right)}{I_{1}^{\prime}\left(-1 /\left(2 \kappa^{*}\right)\right)} & \kappa^{*}<0\end{cases}
$$


It was already shown above that the function $e_{\text {circ }}^{* \max }\left(\kappa^{*}\right)$ is continuous at $\kappa^{*}=0$. When the curvature passes through zero, the two kinds of domains (a hole in an infinite domain vs. a circular tissue domain) are smoothly transformed into each other, which suggests that $e_{\text {circ }}^{* \max }\left(\kappa^{*}\right)$ should be even continuously differentiable. Returning to the physics of the problem, heuristically, the effect of boundaries of different shape should be more or less equivalent in the low curvature regime, as long as their global shape guarantees that other parts of the boundary are several $\lambda$ away from the low curvature site, in order no to disturb the depolarization pattern. As this is the case for the circular boundaries considered here, equation (3.39) should be valid for other kinds of boundaries in the regime $\kappa^{*} \ll 1$.

This universal curvature dependence for small deviations from a flat boundary of curvature zero can be calculated by approximating $I_{n}$ as

$$
I_{n}(x) \approx \sqrt{\frac{\pi}{2 x}} \exp (-x)\left(1-\frac{4 n^{2}-1}{8 x}+\ldots\right)
$$

for large arguments $x=-1 /\left(2 \kappa^{*}\right)$ (equation 9.7.1 in [222], pp. 377-378, truncated after linear order term in parentheses). Using again $I_{n}^{\prime}=\left(I_{n-1}+I_{n+1}\right) / 2$ in the denominator of the negative curvature part of equation $(3.39)$, the depolarization close to zero curvature can be written as

$$
e_{\text {circ }}^{* \max }\left(\kappa^{*}\right)=\frac{2 I_{1}(x)}{I_{0}(x)+I_{2}(x)} \approx \frac{2(1-3 / 8 x)}{2+1 / 8 x-15 / 8 x}=1-\frac{\kappa^{*}}{1+\frac{7}{4} \kappa^{*}} \quad \text { for }\left|\kappa^{*}\right| \ll 1, \kappa^{*}<0 .
$$

A similar calculation for the positive curvature part of equation 3.39), i.e. for $\left|\kappa^{*}\right| \ll 1$, $\kappa^{*}>0$, leads to exactly the same result, meaning that the continuation of equation (3.39) is actually continuously differentiable at $\kappa^{*}=0$ with

$$
\left.\frac{\mathrm{d} e^{* \max }}{\mathrm{d} \kappa^{*}}\right|_{\kappa^{*}=0}=-1 \quad \text { or } \quad e^{* \max } \approx 1-\kappa^{*} \quad \text { for }\left|\kappa^{*}\right| \ll 1
$$

Note that the suffix "circ" was omitted here to emphasize that, according to the above line of thoughts, this result should hold in general for low-curvature parts of boundaries with perpendicular electric fields. In the original units, the slope of the maximum depolarization with respect to boundary curvature is

$$
\left.\frac{\mathrm{d} e^{\max }}{\mathrm{d} \kappa}\right|_{\kappa=0}=-\lambda^{2}|\mathbf{E}|
$$

In a substrate of constant $\lambda$ that has boundaries of different curvature $\kappa$, or in artificiallycultured tissue with well-controlled boundary curvature, this relation opens a way to measure the electrotonic constant $\lambda$ : With an estimate of the left hand side of equation 3.43 from multiple measurements of the depolarization close to boundaries of different curvatures and the known electric field strength, a value of $\lambda^{2}$ can be obtained, which permits a high-accuracy estimate of $\lambda$.

In figure 3.27, the maximum depolarization for circular boundaries as given by equation 3.39 is plotted against the normalized mean curvature $\kappa^{*}=\kappa \lambda$ of the boundary, where positive curvature (gray line) refers to a concave medium and negative curva- 


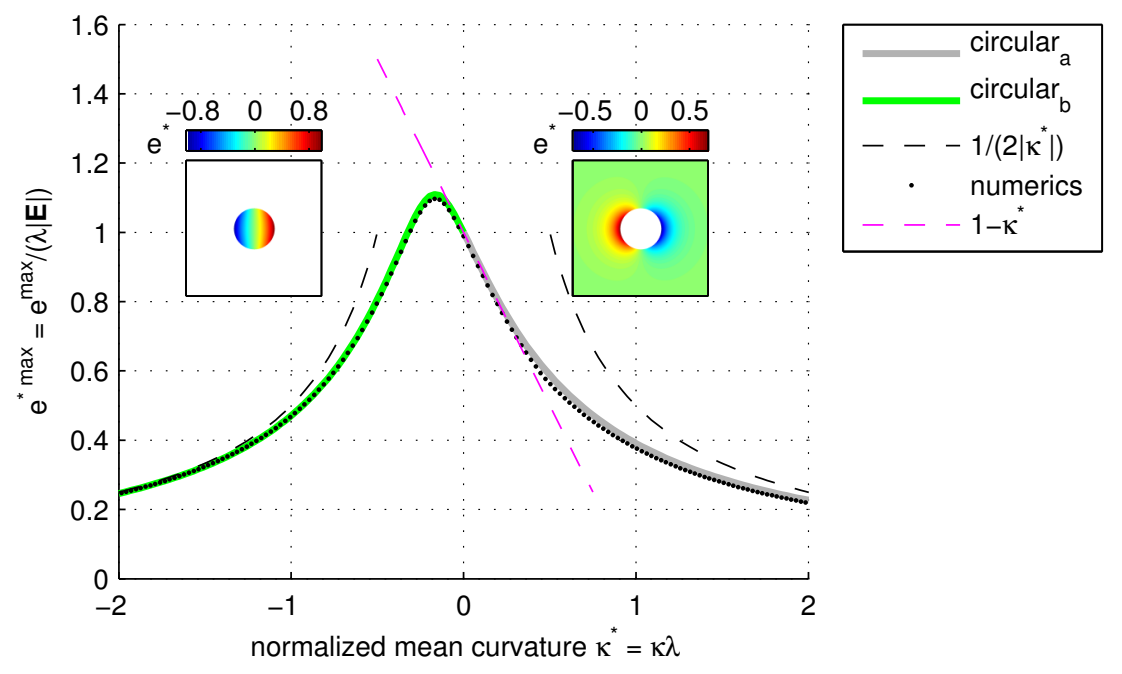

Figure 3.27: Maximum depolarization for circular boundaries. The normalized maximum depolarization $e_{\text {circ }}^{* \text { max }}$ is plotted against the normalized mean curvature $\kappa^{*}=\kappa \lambda$ as predicted by equation (3.39). The dots denote values obtained in numerical simulations with the phase field method as indicated in section 3.2.2. The space step $h$ for these simulations was chosen such that $h<R / 12$ is fulfilled for all cases, where $R$ is the radius of the tissue domain or obstacle. The slightly lower numerical values are due to the blurred interface used with the phase field method (cf. section 2.1.5). The tangent at $\kappa^{*}=0$ from equation (3.42) is plotted as a magenta dashed line. Approximations from equations (3.44) and (3.47) which become exact for $\left|\kappa^{*}\right| \gg 1$ are plotted as black dashed lines. Insets show examplary depolarization patterns for the two branches of the curve (see figure 3.26 for additional examples). 
ture (green line) refers to a convex medium. There are two interesting features, which were not visible in previous studies due to their limitation to positive curvatures: firstly, the non-monotonicity for negative curvature means that there is an "optimal" curvature for which the effect of the electric field is largest. This maximum of equation (3.39) is $e^{* \max }=1.1096 \ldots$ and occurs at $\kappa^{*}=-0.16359 \ldots$, which yields an optimal radius of $R=(3.056 \ldots) \lambda$ for circular tissue domains (or cylinders, according to section 3.2.4). Secondly and more fundamentally, the maximum depolarization can exceed the theoretical limit of $\lambda|\mathbf{E}|$, as calculated in reference [127], meaning that some boundaries with negative curvature will induce excitation for a smaller electric field strength than all boundaries with positive curvature. In the vicinity of $\kappa^{*}=0$, this is already universally predicted by equation (3.42). For the circular boundaries considered here, it is the case in the range from $\kappa^{*}=-0.3236 \ldots$ to $\kappa^{*}=0$, corresponding to a tissue radius from $R=(1.5451 \ldots) \lambda$ to $R=\infty$.

\section{Small tissue domains}

The decline of the maximum depolarization towards large negative curvatures can be attributed to the diffusive interaction with the hyperpolarized area at the opposite boundary like in the one-dimensional case in section 3.2.7. Analogously, the asymptotic shape of the depolarization pattern for a small circular tissue domain can directly be deduced from equation (3.16a): large normalized mean curvatures can by reached by letting $\lambda \rightarrow \infty$ in a domain of finite curvature. Fixing the center point $\mathbf{x}=0$ of the circular domain at $e=0$ because of symmetry, for large $\lambda$, a simple gradient $e=\mathbf{E} \cdot \mathbf{x}$ is a solution of equation (3.16a) and automatically fulfills the boundary condition equation (3.16b). Thus, for small domains, the maximum depolarization is just

$$
e_{b}^{\max }=|\mathbf{E}| R \quad \text { for } R \ll \lambda .
$$

In fact, as the boundary condition equation $(3.16 \mathrm{~b})$ is fulfilled by the asymptotic solution $e=\mathbf{E} \cdot \mathbf{x}$ independently of the normal vector $\mathbf{n}$, this trend holds for arbitrarily shaped tissue small domains $\mathcal{D}$ with a spatial extent smaller than $\lambda$. As an arbitrary $\mathcal{D}$ lacks the symmetry to argue that $e=0$ at the center, it might at first seem that $e=\mathbf{E} \cdot \mathbf{x}+c$ with any $c \in \mathbb{R}$ is a solution. Physically, it is clear that the deviation $e$ from the resting potential must have a defined value. For a finite, but large $\lambda, e$ can be gauged by integrating equation (3.16a) over the tissue domain $\mathcal{D}$ :

$$
\begin{aligned}
0 & =\int_{\mathcal{D}} \nabla^{2} e \mathrm{~d} V-\frac{1}{\lambda^{2}} \int_{\mathcal{D}} e \mathrm{~d} V=\oint_{\partial \mathcal{D}} \nabla e \cdot \mathbf{n} \mathrm{d} A-\frac{\operatorname{Vol}(\mathcal{D})}{\lambda^{2}}\langle e\rangle_{\mathcal{D}} \\
& \stackrel{[3.16 b]}{-} \oint_{\partial \mathcal{D}} \mathbf{E} \cdot \mathbf{n} \mathrm{d} A-\frac{\operatorname{Vol}(\mathcal{D})}{\lambda^{2}}\langle e\rangle_{\mathcal{D}}=-\frac{\operatorname{Vol}(\mathcal{D})}{\lambda^{2}}\langle e\rangle_{\mathcal{D}} \\
& \Rightarrow\langle e\rangle_{\mathcal{D}}=0
\end{aligned}
$$

$\langle\cdot\rangle_{\mathcal{D}}$ denotes the spatial average over the tissue domain $\mathcal{D}$. For the solution $e=\mathbf{E} \cdot \mathbf{x}+c$, $\langle e\rangle_{\mathcal{D}}=0$ is fulfilled with $c=0$, if, without loss of generality, the coordinate system is defined such that the center of mass of the tissue domain $\mathcal{D}$ is in the origin. If, additionally, 
the $x$-axis is aligned with the electric field vector, the maximum depolarization is simply $e^{\max }=|\mathbf{E}| l$, where $l$ is the maximum $x$-coordinate within the tissue domain $\mathcal{D}$. This is consistent with equation (3.47) for a circular domain.

According to figure 3.27, in the positive curvature regime, the maximum depolarization also decreases with increasing curvature $\kappa^{*}$. The asymptotic behavior can be derived in a similar way as in the negative-curvature case: since the domain is infinitely large, the gauge condition above is replaced here by the additional condition that the solution is assumed to decay far away from the circular obstacle. The correct solution to $\nabla^{2} e=0$, the asymptotic form of equation (3.16a) for large $\lambda$, is

$$
e_{a}=-\frac{|\mathbf{E}| R^{2}}{r} \cos (\varphi) \quad \text { for } R \ll \lambda
$$

as it is the real part of the holomorphic function $|\mathbf{E}| R^{2} z^{-1}$ and it also fulfills the boundary condition 3.29). Therefore, the maximum depolarization, which occurs again at $\varphi=\pi$ and $r=R$, is

$$
e_{a}^{\max }=|\mathbf{E}| R \quad \text { for } R \ll \lambda
$$

and thus identical to the negative curvature case (3.44). Both (3.47) and (3.44) result in

$$
e_{\text {circ }}^{* \max }\left(\kappa^{*}\right)=\frac{1}{2\left|\kappa^{*}\right|} \quad \text { for }\left|\kappa^{*}\right| \gg 1
$$

for circular boundaries, which is shown as a black dashed line in figure 3.27. This result could also have been obtained by approximating equations (3.33) and (3.34) for small arguments of the Bessel functions.

\subsubsection{Semi-circular protuberances}

For circular tissue boundaries, high curvatures are intrinsically connected to small domains (in the case of negative curvatures) or small holes (for positive curvature). It was shown above that, for small domains, interactions between depolarized and hyperpolarized regions set in that diminish the maximum depolarization in the tissue. Since the universal effect of negative curvature seems to be an increase in the maximum depolarization, as supported by the low curvature approximation (3.42), one can pose the question whether a shape that avoids the proximity of hyperpolarized boundaries would show an even larger response to electric fields.

The shape shown in figure 3.25c) eliminates the compensatory effect by cutting away the hyperpolarized half of the boundary. In figure 3.28 , the maximum depolarization for this shape is plotted against the curvature $\kappa^{*}=\lambda /(2 R)$ of the protuberance. It was calculated purely numerically by the procedure explained in section 3.2 .2 . The curve features a maximum and a minimum for negative and positive curvatures, respectively, and declines towards $\lambda|\mathbf{E}|$ for $\left|\kappa^{*}\right| \rightarrow-\infty$. The maximum $e_{\text {prot }}^{* \max } \approx 1.22$ of the curve is considerably higher than for circular boundaries, in agreement with the absence of the compensatory effect between adjacent regions of de- and hyperpolarization described above. The fact that the depolarization is non-monotonic and approaches $\lambda|\mathbf{E}|$ for large curvatures (in 


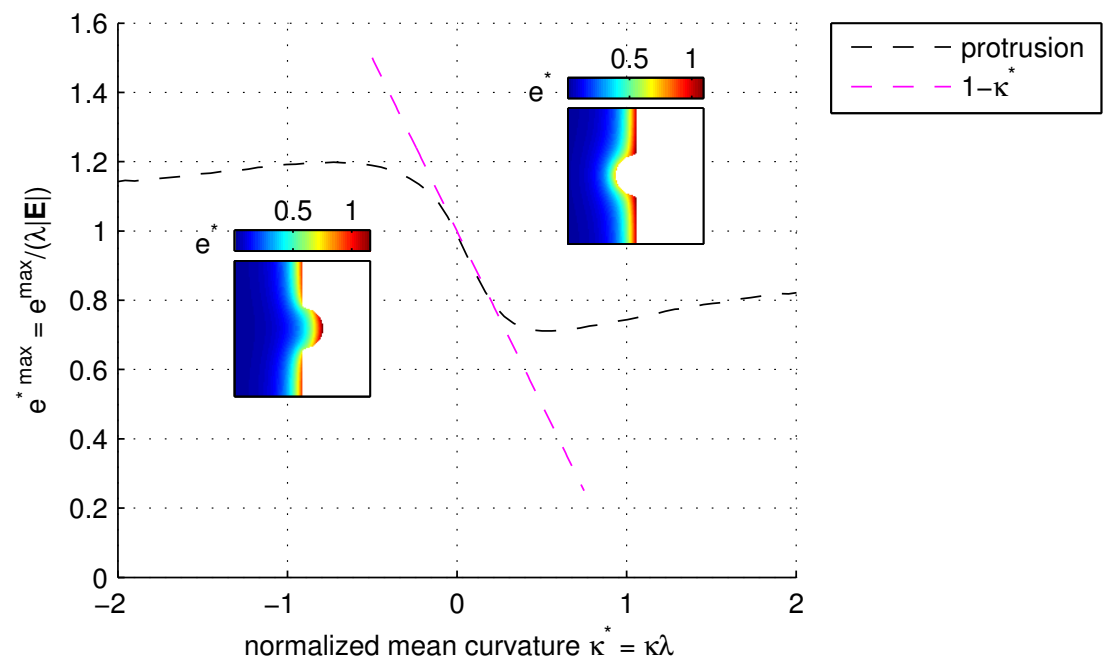

Figure 3.28: Maximum depolarization for semi-circular protuberances. The normalized maximum depolarization $e_{\text {prot }}^{* \max }$ is plotted against the normalized mean curvature $\kappa^{*}=\kappa \lambda$. The low curvature approximation (equation (3.42), dashed line) is tangent to the curve at $\kappa^{*}=0$. All values were obtained in numerical simulations as outlined in section 3.2 .2 . Insets show examplary depolarization patterns for negative and positive curvature.

either direction) is again due to diffusivity: although there is no hyperpolarized boundary nearby, large curvatures imply small protuberances. The membrane potential is smoothed out within an area of characteristic length $\lambda$, such that features much smaller than $\lambda$ cannot be resolved by the dynamics of the system. Therefore, below a certain size of the protuberance, the depolarization pattern approaches that of a straight boundary.

\subsubsection{Parabolic boundaries}

Although there is no interaction between regions of de- and hyperpolarization for semicircular protuberances, the limited effect size is again related to the close connection between the scale of features and the curvature. A shape which does not change qualitatively while assuming higher curvatures is the parabolic tissue domain $\mathcal{D}=\{(x, y) \in$ $\left.\mathbb{R}^{2} \mid x<a y^{2}\right\}$, which has mean curvature $\kappa=a$ at the origin and is shown in figure $3.25 \mathrm{~d}$ ). Analytical calculation of the solution $e_{\text {parab }}(\mathbf{x})$ for the basic equation (3.16) is impeded by the boundary condition (3.16b), which does not assume a simple form in parabolic coordinates. However, the solution $e_{\text {parab }}$ for $\kappa^{*}<0$ (when the domain has finite extent in $y$-direction for any given $x$ ) can be composed as a linear combination of a few terms of the form $\exp \left( \pm x / \lambda_{x, i} \pm y / \lambda_{y, i}\right)$ with different $\lambda_{x, i}$ and $\lambda_{y, i}$ which satisfy $1 / \lambda_{x, i}^{2}+1 / \lambda_{y, i}^{2}=1 / \lambda^{2}$ for all $i$. This ensures that the terms are solutions of equation (3.16a). As for circular boundaries, an electric field in positive $x$-direction is assumed. The solution must be invariant under $y \rightarrow-y$ and $e(\mathbf{x})$ must vanish for $x \rightarrow-\infty$ (for every fixed $y$ ). To satisfy these conditions, valid solutions must have equal coefficients for terms with $+\lambda_{y, i}$ and $-\lambda_{y, i}$, and $\operatorname{Re}\left(\lambda_{x, i}\right)>0$ must be fulfilled.

As it turns out numerically, even terms with $\lambda_{x, i}, \lambda_{y, i} \in \mathbb{R}$ suffice to compose an accurate 


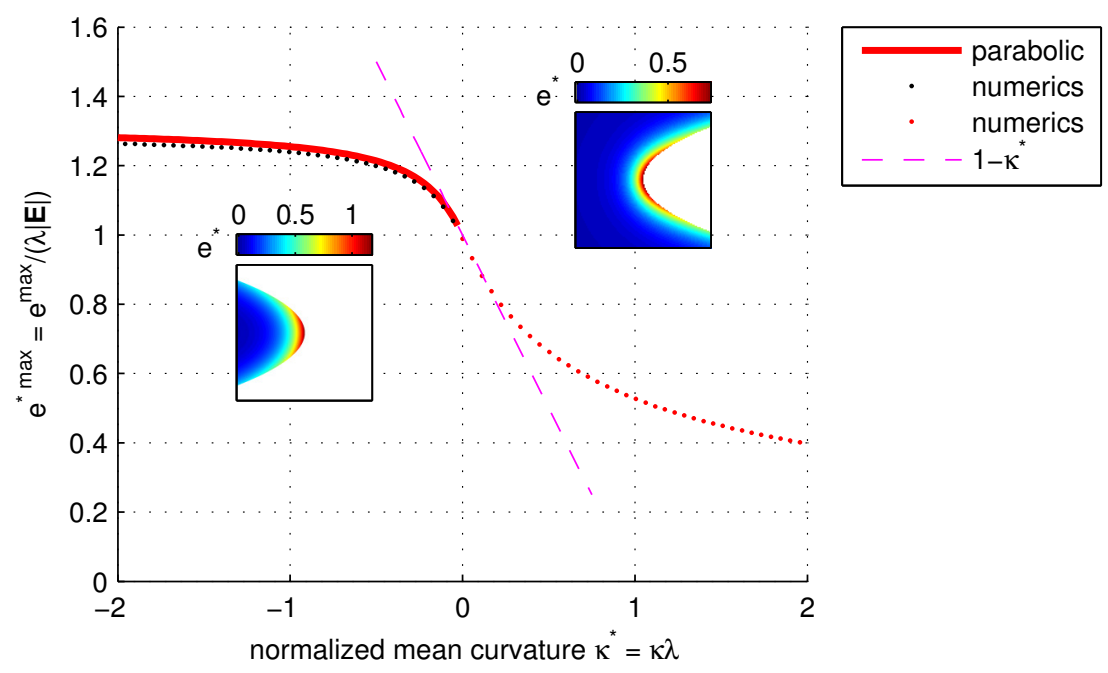

Figure 3.29: Maximum depolarization for parabolic boundaries. The normalized maximum depolarization $e_{\text {parab }}^{* \max }$, as obtained by the ansatz $(3.49)$, is plotted against the normalized mean curvature $\kappa^{*}=\kappa \lambda$ as a red line. The dots indicate numerical simulations for the positive curvature regime and to verify the semi-analytical solution (see section 3.2.2). Again, the slightly lower numerical values are due to the blurred interface used with the phase field method (cf. section 2.1.5). The low curvature approximation (3.42 is plotted as a dashed line. Insets show examplary depolarization patterns for negative and positive curvature.

solution. In summary, we make the ansatz:

$$
\begin{aligned}
e_{\text {parab }}(\mathbf{x}) & =\sum_{i=1}^{N} c_{i} e_{i}(\mathbf{x}) \\
\text { with } e_{i}(\mathbf{x}) & =\exp \left(x / \lambda_{x, i}\right) \cosh \left(y / \lambda_{y, i}\right),
\end{aligned}
$$

where $\lambda_{x, i}, \lambda_{y, i}>0$ for all $i$. Because of the form of the single terms $(3.50), e_{\text {parab }}(\mathbf{x})$ will be an exact solution of equation (3.16a). Furthermore, the coefficients $c_{i}$ are chosen such that equation $3.16 \mathrm{~b}$ is approximately satisfied. This is done by choosing a set of points $\mathbf{x}_{j}=\left(a y_{j}^{2}, y_{j}\right)$ on the boundary and expressing the boundary condition of equation $(3.16 \mathrm{~b})$ in these cartesian coordinates:

$$
-\left.1 \cdot \frac{\partial e}{\partial x}\right|_{\left(x_{j}, y_{j}\right)}+\left.2 \kappa y_{j} \cdot \frac{\partial e}{\partial y}\right|_{\left(x_{j}, y_{j}\right)}=-E_{x} \quad \forall j .
$$

The partial derivatives can be calculated analytically by substituting equation 3.49 for $e(\mathbf{x})$, which turns equation (3.51) into a system of linear equations for the coefficients $c_{i}$. This system was solved for the most sparse solution. The relative error of the solution was calculated as the maximum deviation of the right hand side of equation (3.51) from its left hand side, divided by $E_{x}$, using points $\tilde{\mathbf{x}}_{j}$ on the boundary with a density 10 times higher than the $\mathbf{x}_{j}$, for which the solution was calculated. For the results shown here, this relative error never exceeded $10^{-10}$, which is why the solution can be characterized as "semi-analytical".

The resulting maximum depolarization $e_{\text {parab }}^{*} \max$ is plotted as a red line in figure 3.29 . Despite 


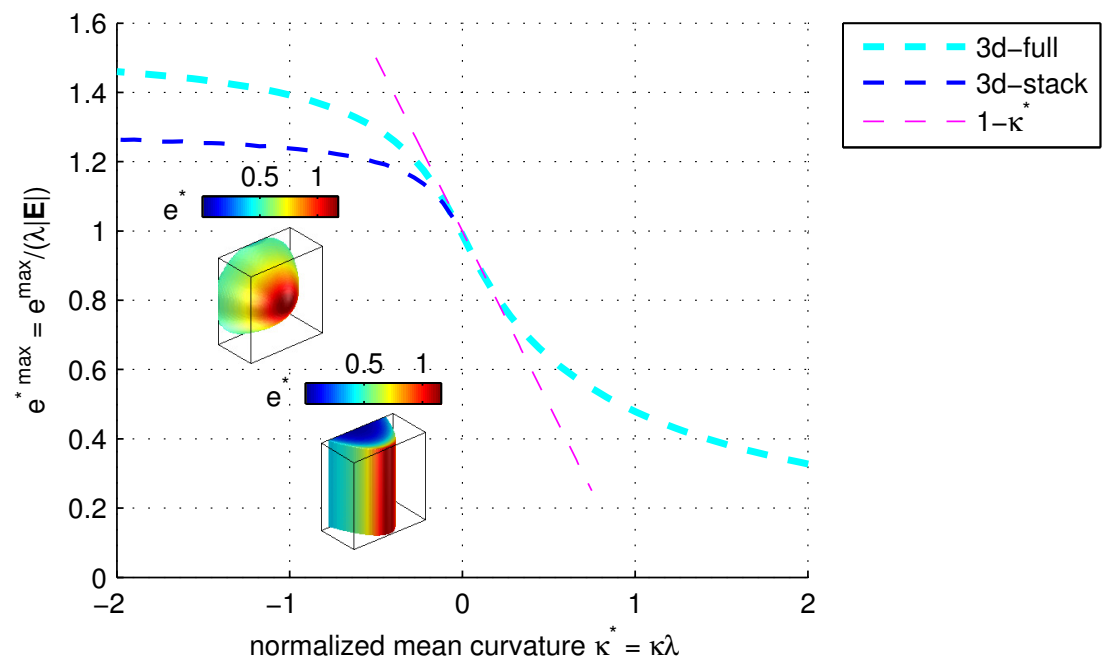

Figure 3.30: Maximum depolarization for three-dimensional boundaries. The normalized maximum depolarization $e_{\text {parab }}^{* \max }$ is plotted against the normalized mean curvature $\kappa^{*}=\kappa \lambda$ of the parabolic boundaries. All values were obtained in numerical simulations according to section 3.2 .2 . Insets show examplary depolarization patterns for the negative-curvature regime.

the non-circular shape of the domain, the behavior of the curve close to $\kappa^{*}=0$ is predicted correctly by the approximation (3.42), which confirms its universality. In contrast to the other shapes considered so far, $e_{\text {parab }}^{* \max }$ is monotonic. From the finite part of the curve that was calculated, it cannot be concluded whether there will be a limiting value for large $\kappa^{*} \rightarrow-\infty$. However, the steepness of the curve decreases quickly towards larger negative curvatures. Thus, the most apparent difference in the reaction of this kind of boundary to electric fields should be noted up to curvatures of the order of $-1 / \lambda$.

For positive curvatures, the maximum depolarization was determined numerically as outlined in section 3.2.2. Figure 3.29 indicates that the maximum depolarization in this regime decreases towards larger positive curvatures, as does that of circular boundaries in figure 3.27. However, the comparative plot of figure 3.31 shows that the decline for parabolic boundaries is considerably more shallow. Similar to the scenario for negative curvatures, the stronger electric-field induced depolarization for parabolic boundaries can be attributed to the absence of a close hyperpolarized boundary, which is present for circular boundaries (c.f. right inset of figure 3.27). There, once the distance of the hyperpolarized part of the boundary to the point of maximum depolarization becomes comparable to $\lambda$, the electrotonic interaction leads to a reduction of the effect strength, in addition to that one caused purely by the curvature of the boundary.

\subsubsection{Inherently three-dimensional boundaries}

Numerical results for two three-dimensional tissue domains are plotted as blue and cyan dashed lines in figure 3.30. For the blue line, $\mathcal{D}=\left\{(x, y, z) \in \mathbb{R}^{3} \mid x<a y^{2}\right\}$, i.e., $\mathcal{D}$ is a three-dimensional extension of figure $3.25 \mathrm{~d}$ ) with zero curvature in $z$-direction. The two principal curvatures $\kappa_{1}=2 a$ and $\kappa_{2}=0$ lead to a mean curvature of $\kappa=a$ as in the 


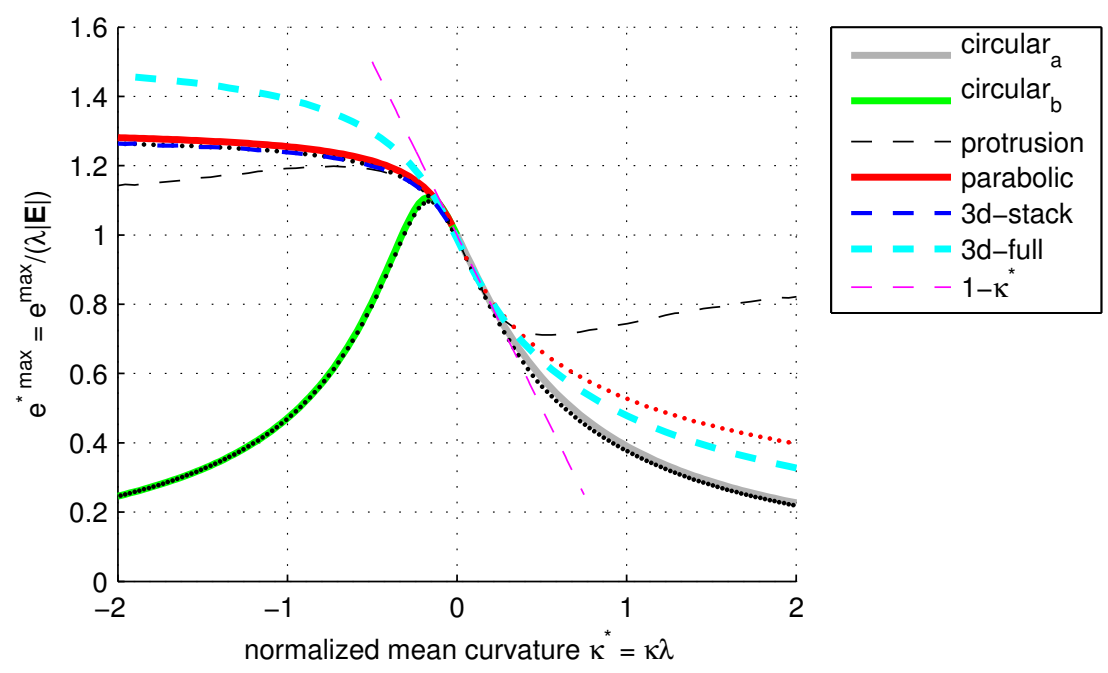

Figure 3.31: Summary: Depolarization for diffferent boundary types. The normalized maximum depolarization $e^{* \max }$ for all shapes considered in this study is plotted against the normalized mean curvature $\kappa^{*}=\kappa \lambda$. Colors and line styles of the original plots were maintained.

two-dimensional case. This corresponds to the scenario considered in section 3.2.4, which is why the maximum depolarization $e_{3 \mathrm{~d} \text {-stack }}^{*}$ is exactly the same as in the two-dimensional case (see summarizing figure 3.31). This confirms numerically that, as derived in section 3.2.4 three-dimensional extensions of the shapes considered so far will yield the same depolarization pattern: circular domains become cylinders, circular obstacles are equivalent to cylindrical obstacles and the semi-circular protuberances cannot be differentiated from cross-sections through half-cylinders attached to a flat surface of the tissue.

However, if the added dimension introduces additional curvature, this can enhance (or diminish) the observed depolarization. The tissue domain defined by $\mathcal{D}=\{(x, y, z) \in$ $\left.\mathbb{R}^{3} \mid x<a\left(y^{2}+z^{2}\right)\right\}$ corresponds to the parabolic shape rotated around the $x$ axis. Its principal curvatures at the origin are $k_{1}=2 a$ and $k_{2}=2 a$, resulting in a mean curvature of $\kappa=2 a$. The resulting maximum depolarization at the tip of the paraboloid $e_{3 \mathrm{~d} \text {-full }}^{*}$ is shown as a cyan curve in figure 3.30. For larger negative curvatures, the additional curved dimension increases the induced depolarization. This is an inherent effect of the threedimensional topology, since all curves are plotted against the mean curvature. In contrast, towards zero curvature, the maximum depolarization asymptotically behaves according to the conjecture (3.42), meaning that for this regime, the mean curvature alone determines the strength of depolarization. This agreement between native three-dimensional boundaries and two-dimensional boundaries close to $\kappa^{*}=0$ is also a reassurement that the mean curvature is a suitable mathematical quantity to characterize the boundary.

\subsubsection{Boundary effects in full numerical simulations}

The results in figure 3.31 were obtained by solving equation (3.16), which is a low-fieldstrength, mono-domain, steady-state approximation. As indicated at the beginning of section 3.2 , electric-field induced membrane potential changes are thought to be important during stimulation, e.g., in an effort to terminate arrhythmias. In this situation, the 
a)

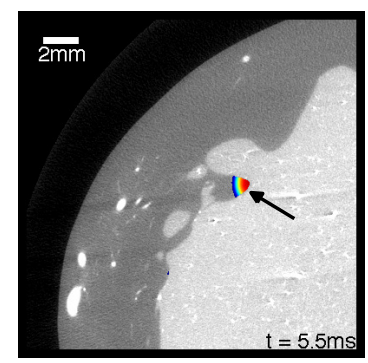

b)

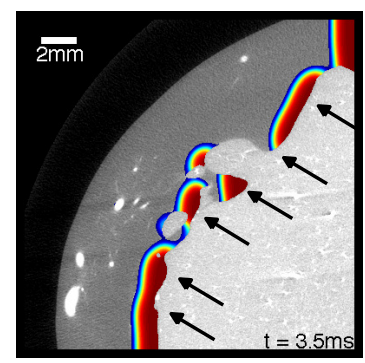

c)

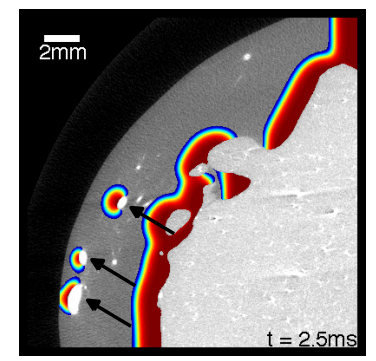

$-80 \mathrm{mV}$
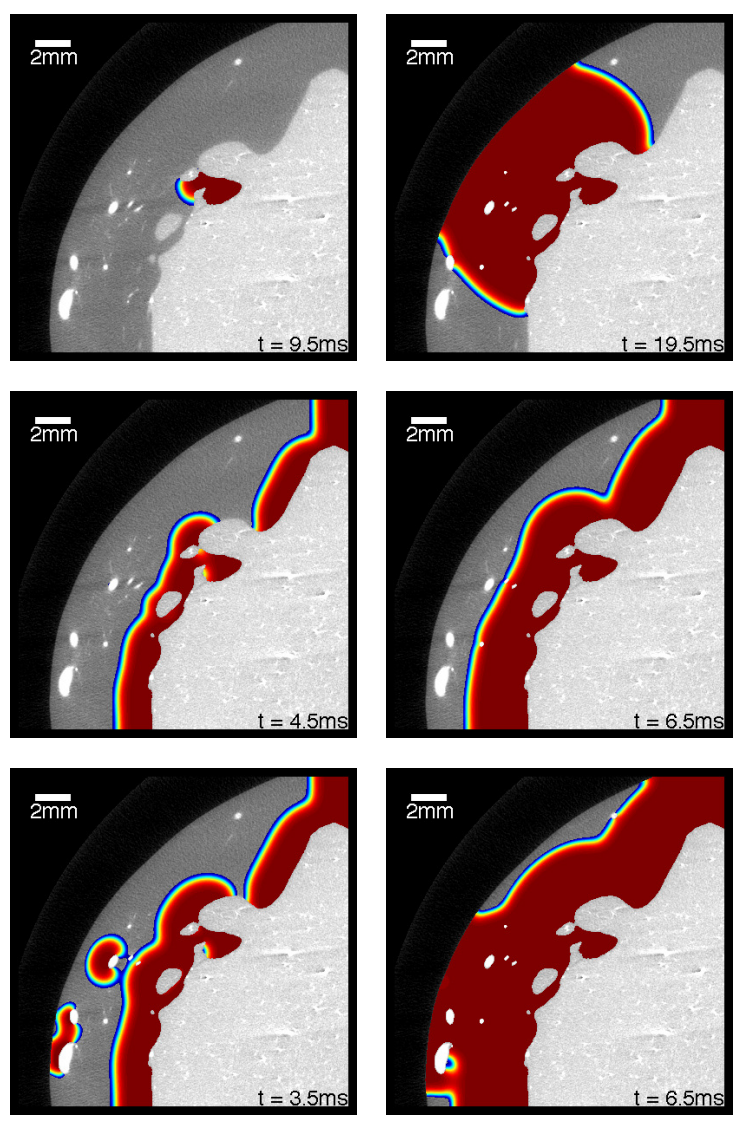

Figure 3.32: Electric-field stimulation in a two-dimensional simulation. Numerical simulation of a $5 \mathrm{~ms}$ monophasic shock at a field strength E, resulting in curvaturedependent activation. The geometry of a cross section of a left ventricular rabbit wall was obtained from $\mu \mathrm{CT}$ and implemented using the phase field method with the modified boundary condition (3.16b). a) $\mathrm{E}=0.2 \mathrm{~V} / \mathrm{cm}$ : wave emission is observed at a protuberance with large negative curvature, while depolarization at the boundary remains below threshold. b) $\mathrm{E}=0.4 \mathrm{~V} / \mathrm{cm}$ : excitation of almost the entire tissue boundary results in a quasi-planar wave. c) $\mathrm{E}=1.0 \mathrm{~V} / \mathrm{cm}$ : Internal boundaries with positive curvature (blood vessels indicated by arrows) emit waves. Simulations were carried out using the FentonKarma model with parameter set FK1 (table A.2, page 177) and isotropic diffusion of $1 \mathrm{~cm}^{2} / \mathrm{s}$. The time step is $\Delta t=2.5 \mu \mathrm{s}$, the space step $h=50 \mu \mathrm{m}$. Figure reproduced from [218. 
purpose of the stimulation is to induce new waves in the cardiac muscle that act as control stimuli. However, induction of the waves ultimately requires a superthreshold stimulation, a process not covered by the low-field strength approximation. Additionally, finite pulse durations are used in reality, such that, even if the membrane dynamics is assumed to be passive up to the excitation threshold, the asymptotic depolarization value might not be reached. Therefore, it is important to validate the approximation in more realistic numerical simulations. This was done on the complex geometry of the left ventricular wall obtained from $\mu \mathrm{CT}$. The full, non-linear, time-dependent Fenton-Karma model was simulated, with equation (3.16b) instead of the usual no-flux boundary condition. In contrast to the numerical simulations carried out so far (cf. section 3.2.2), now, fieldstrengths that are capable of evoking action potentials are used, therefore leaving the scope of the linear (passive membrane) approximation introduced in section 3.2.1. The results shown in figure 3.32 qualitatively reproduce the mechanisms described above: A large protuberance on the endocardium is activated at a lower field strength than the flat part of the endocardium. This finding agrees with the cell culture experiments shown in figure 3.22 and the increased sensitivity of the negative-curvature regime demonstrated by figure 3.31. Inner boundaries such as blood vessels are being activated at higher field strengths, since they correspond to the regime of $\kappa^{*}>0$ in figure 3.31, for which the depolarization is generally lower. Good agreement with the theoretical results shown in figure 3.31 suggests that other effects which might depend on the geometry such as the liminal area [223] only play a secondary role.

The action of different kinds of boundaries as wave emitting sites in a realistic threedimensional setting is illustrated in figure 3.33. The Fenton-Karma model was used with parameters obtained by J. SchröDER-SCHETELIG from a fitting procedure of the model to measured restitution curves in rabbit hearts [224]. A high field strength was chosen, such that the multitude of boundary reactions becomes visible (corresponding to the case of figure $3.32 \mathrm{k})$ ). The different kinds of boundaries leading to separate activations include papillary muscles (arrow A), endocardial undulations (arrow B) and blood vessels (arrows $\mathrm{C}, \mathrm{D}$ and E). In reality, many more (smaller) blood vessels would be activated which are not resolved accurately by the numerical simulation due to resolution limitations $(58 \mu \mathrm{m})$.

\subsubsection{Influence of finite pulse duration}

From the numerical simulations shown in figure 3.32 , it seems that the maximum depolarizations of different kinds of boundaries obtained from the low field strength, steady-state approximation (figure 3.31) can qualitatively be translated to realistic settings based on a finite pulse duration and complex geometries. However, this does not mean that quantitative results like the optimal curvature for circular boundaries (peak of the green line in figure 3.31) are preserved as well. A priori, it is not clear how a spatial property like the boundary curvature interacts with processes that are thought to be determined by local properties such as the subthreshold membrane time constant ( $\tau_{0}$ in the case of the Fenton-Karma model). Implicitly, this time constant was included in the electrotonic constant $\lambda$ introduced in section 3.2.1, but the results presented above yield no information on the time scale on which the membrane potential approaches the computed steady-state 

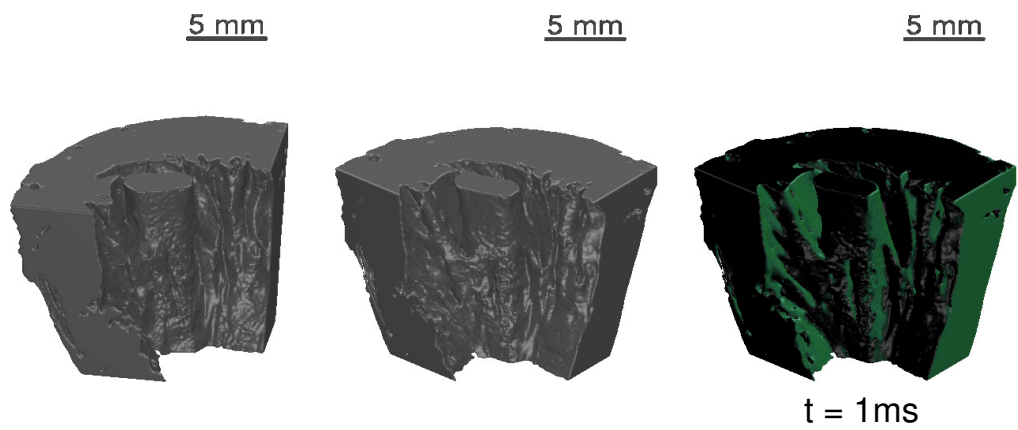

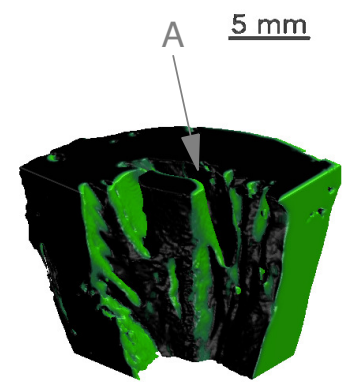

$\mathrm{t}=3 \mathrm{~ms}$

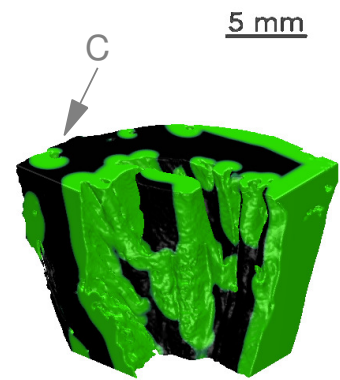

$\mathrm{t}=10 \mathrm{~ms}$

$\underline{5 \mathrm{~mm}}$

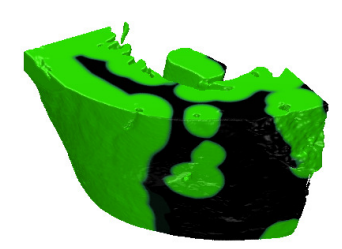

$\mathrm{t}=14 \mathrm{~ms}$

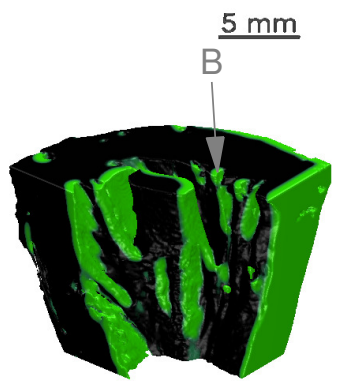

$\mathrm{t}=5 \mathrm{~ms}$

$\underline{5 \mathrm{~mm}}$

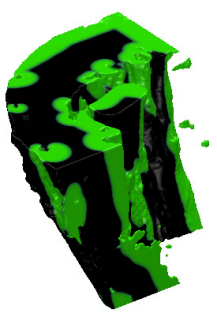

$\mathrm{t}=10 \mathrm{~ms}$

$\underline{5 \mathrm{~mm}}$

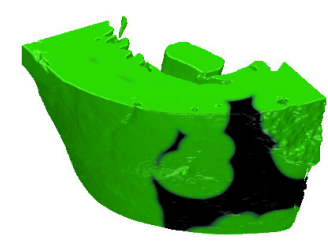

$\mathrm{t}=18 \mathrm{~ms}$
$5 \mathrm{~mm}$

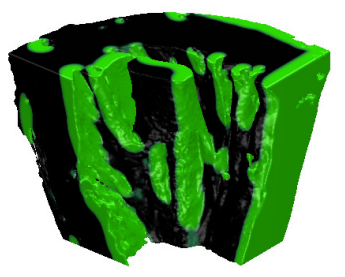

$\mathrm{t}=7 \mathrm{~ms}$

$\underline{5 \mathrm{~mm}}$

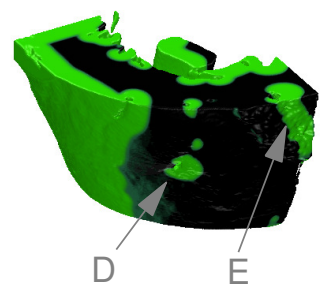

$\mathrm{t}=10 \mathrm{~ms}$

$\underline{5 \mathrm{~mm}}$

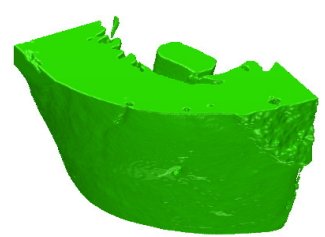

$\mathrm{t}=30 \mathrm{~ms}$

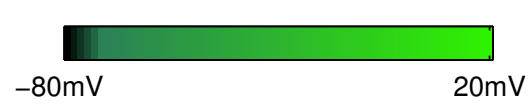

Figure 3.33: Electric-field stimulation in a three-dimensional simulation. Numerical simulation of a $10 \mathrm{~ms}$ monophasic shock at a field strength of $2 \mathrm{~V} / \mathrm{cm}$. The geometry of a piece of a left ventricular rabbit wall was obtained from $\mu \mathrm{CT}$ and implemented using the phase field method with the modified boundary condition (3.16b). The electric field is approximately directed into the image plane on the second snapshot at $t=10 \mathrm{~ms}$ (third row, second column). Simulations were carried out using the Fenton-Karma model with parameter set FK4 (table A.2, page 177) and isotropic diffusion of $0.1 \mathrm{~cm}^{2} / \mathrm{s}$. A different color code was chosen for visualization purposes. 
depolarization patterns.

As a first step towards determining the additional mechanisms which come into play when pulses with finite duration are used to bring the membrane potential above the excitation threshold, circular boundaries (of positive and negative curvature) shall be considered in this section using numerical simulations. A simple, analytical way to obtain an estimate for the field strength required to induce excitation at such a boundary from the theory above is to calculate, at what field strength the maximum depolarization of the asymptotic membrane potential distribution (cf. figure 3.27) reaches the excitation threshold. Denoting the excitation threshold by $e_{t}$ (relative to the resting potential; in the Fenton-Karma model, $e_{t} \approx u_{c}$ ), this asymptotic required field is thus determined by the condition

$$
\begin{aligned}
E_{\text {req }}^{\text {asymptotic }} \lambda e_{\text {circ }}^{* \max }\left(\kappa^{*}\right) & =e_{t} \\
\Rightarrow E_{\text {req }}^{\text {asymptotic }}\left(\kappa^{*}\right) & =\frac{e_{t}}{\lambda} \frac{1}{e_{\text {circ }}^{* \max }\left(\kappa^{*}\right)},
\end{aligned}
$$

where $e_{\text {circ }}^{* \max }\left(\kappa^{*}\right)$ is the unified maximum depolarization for circular boundaries with positive and negative curvatures of equation (3.39).

Alternatively, the critical electric field strength $E_{\text {req }}$ can be measured directly in numerical simulations of the Fenton-Karma model by employing a procedure of nested intervals. This way $E_{\text {req }}$ can be determined for each normalized curvature $\kappa^{*}$ to arbitrary (but finite) accuracy. Again, the parameter set fitted to rabbit restitution data was used (FK4, see table A.2, page 177). The same adaptive numerical resolution as for the numerical results in figure 3.27 was chosen for different curvatures, to ensure that the small circular boundaries for large $\left|\kappa^{*}\right|$ are well resolved by the phase field method and to guarantee comparability. In figure 3.34, subthreshold and superthreshold stimulations with rectangular biphasic pulses for two exemplary curvatures are shown. The depicted simulations correspond to the final step of the nested interval procedure, meaning that subthreshold and superthreshold field strengths for each curvature represent lower and upper bounds for the required field strength $E_{\text {req }}$, respectively. The determined required field strength $E_{\text {req }}$ for a whole range of curvatures is plotted in figure $3.35 \mathrm{a}$ ). The blue symbols denote the numerical results for three different pulse shapes identical to those used in experiments (see figure 2.12, page 74 ). The pulse duration is $T_{\text {pulse }}=10 \mathrm{~ms}$ in all cases and $E_{\text {req }}$ is the minimum peak field strength (corresponding to $E_{\max }$ in figure 2.12), for which the stimulation results in an activation. Asymptotic theoretical values for $E_{\text {req }}\left(\kappa^{*}\right)$ from equation (3.52) are plotted as a red line.

Figure $3.35 \mathrm{a}$ ) indicates that, generally, all pulse shapes require an equal or higher field strength for activating the boundary than estimated by the asymptotic approximation. This is expected because the finite pulse duration prevents the membrane potential from reaching its limiting value 15 While monophasic and biphasic pulses do not lead to quantitatively very different results, the required peak field strength for the biphasic truncated exponential pulse shape is considerably larger. However, one has to bear in mind that in each half of the pulse, much less total charge is injected into the domain than for a rect-

\footnotetext{
${ }^{15}$ Note that, despite the adaptive space step $h$ of the simulations, the numerical realization of the boundaries using the phase-field method slightly underestimates the electric field-induced effect in the asymptotic case (see figure 3.27). Assuming this trend holds for finite pulse duration, the critical field strength in the corresponding field strength range shown in figure 3.35 could be slightly too high.
} 
a)

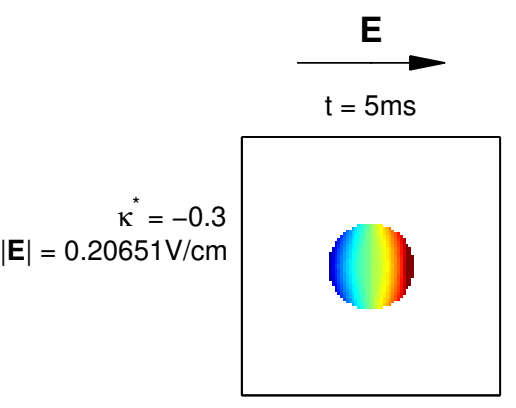

b)

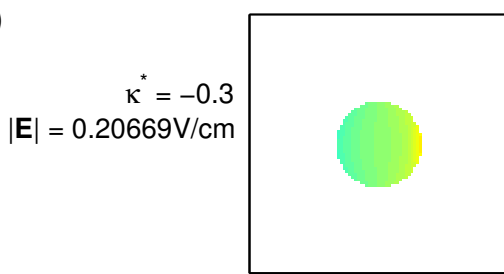

c)

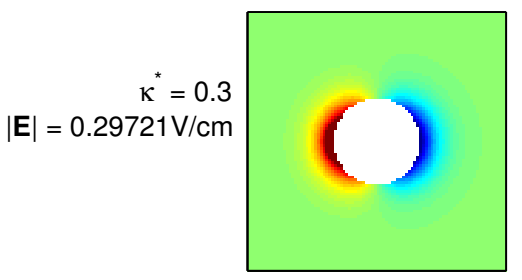

d)

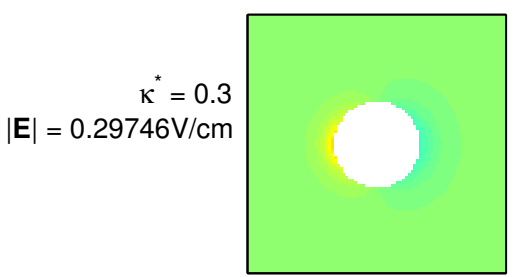

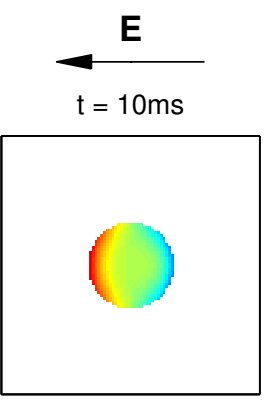
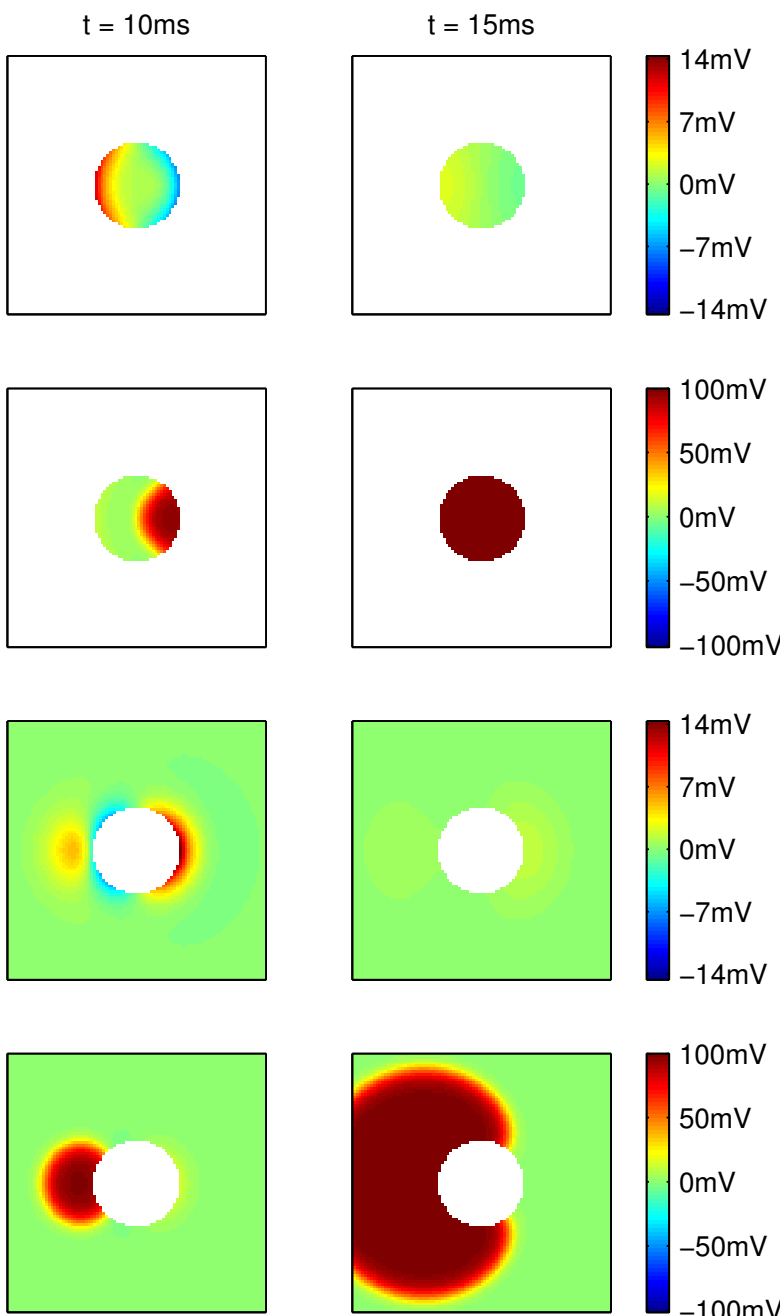

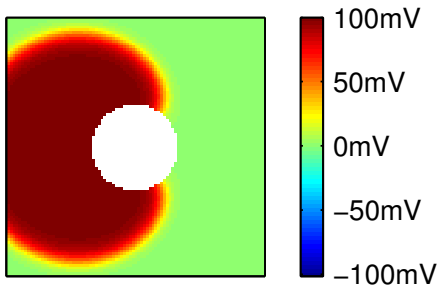

Figure 3.34: Critical field strength for biphasic rectangular pulses: small curvatures. In each row, the deviation from the resting membrane potential $5 \mathrm{~ms}, 10 \mathrm{~ms}$ and $15 \mathrm{~ms}$ after the beginning of the stimulation with a biphasic rectangular electric field pulse (cf. figure 2.12, page 74) is shown. The total pulse duration is $T_{\text {pulse }}=10 \mathrm{~ms}$ in all cases. The first two columns correspond to snapshots after each half of the pulse (electric field direction during the preceding $5 \mathrm{~ms}$ indicated by arrow above each column). Note the different color codes for subthreshold and superthreshold cases. a), b) Subthreshold and superthreshold stimulation, respectively, of a circular tissue domain of normalized mean curvature $\kappa^{*}=-0.3$. c), d) Subthreshold and superthreshold stimulation, respectively, of a tissue domain containing a circular obstacle with a normalized mean curvature of $\kappa^{*}=0.3$. Subthreshold stimulation in a) and c) does not activate the tissue and the membrane potential decays quickly back to the resting value. For b) and d), superthreshold stimulation leads to an activation induced by the first half of the biphasic pulse. Simulations were carried out using the Fenton-Karma model with parameter set FK4 (table A.2. page 177). 
a)

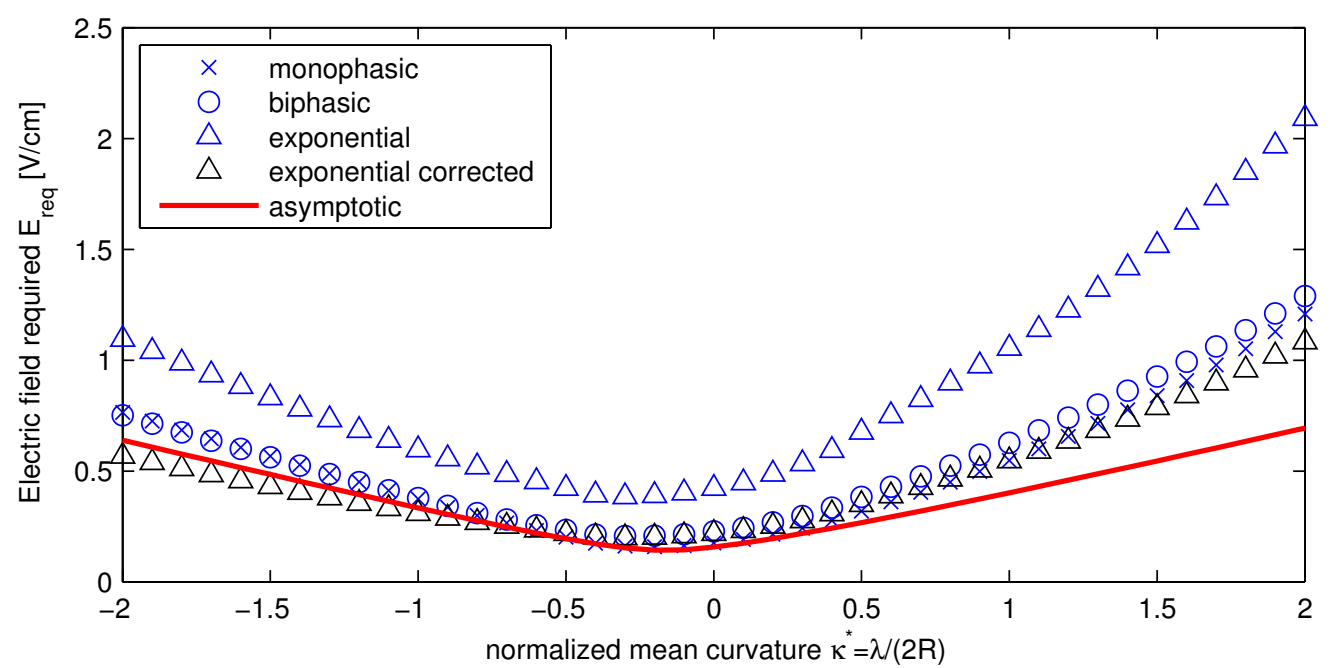

b)

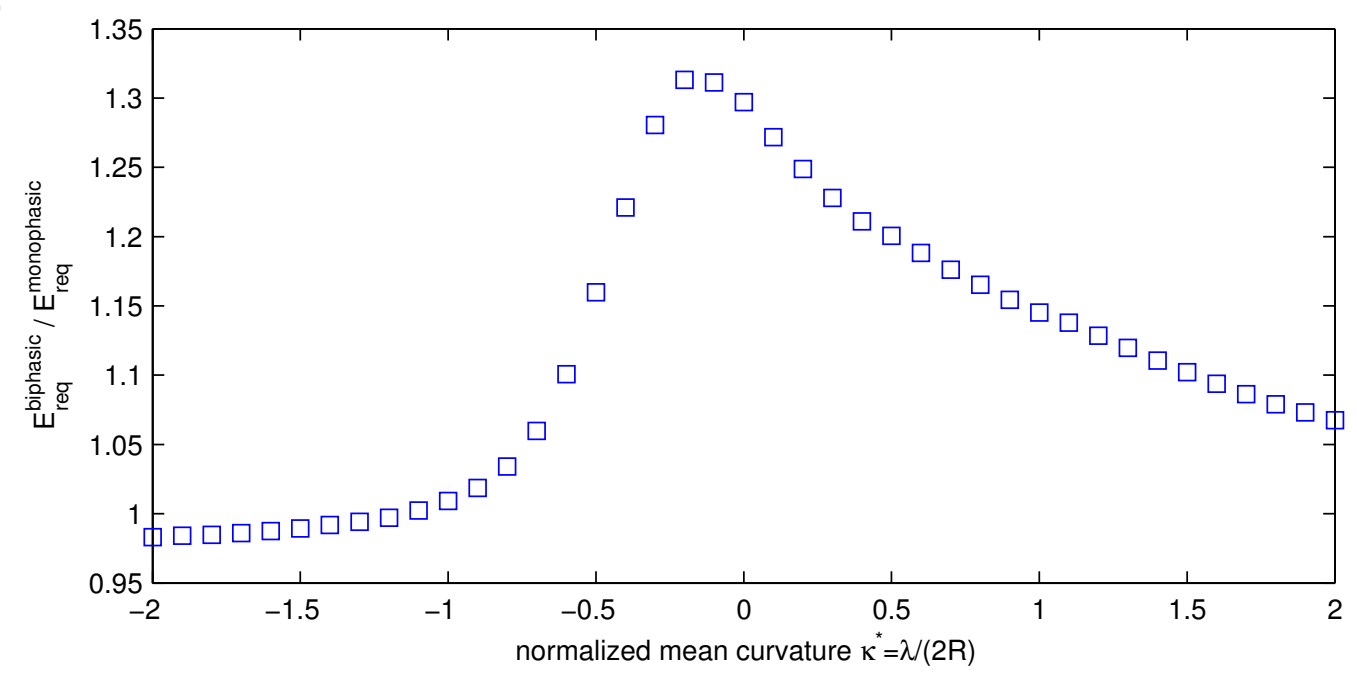

Figure 3.35: Electric-field pulses of finite duration and different shapes at circular boundaries. a) Field strength required to induce a wave from a circular boundary plotted against the normalized mean curvature $\kappa^{*}$ of the boundary. The three blue symbols correspond to the pulse shapes depicted in figure 2.12, page 74 , where the pulse duration is $T_{\text {pulse }}=10 \mathrm{~ms}$ for all pulse shapes and $\tau_{\text {decr }}=3.33 \mathrm{~ms}$ for the truncated exponential wave form. Black triangles are obtained from blue triangles by multiplication with the correction factor (3.53). The red line denotes the asymptotic expected minimum field strength, see equation (3.52). b) Ratio between the required field strength from a) for biphasic and monophasic pulses. 


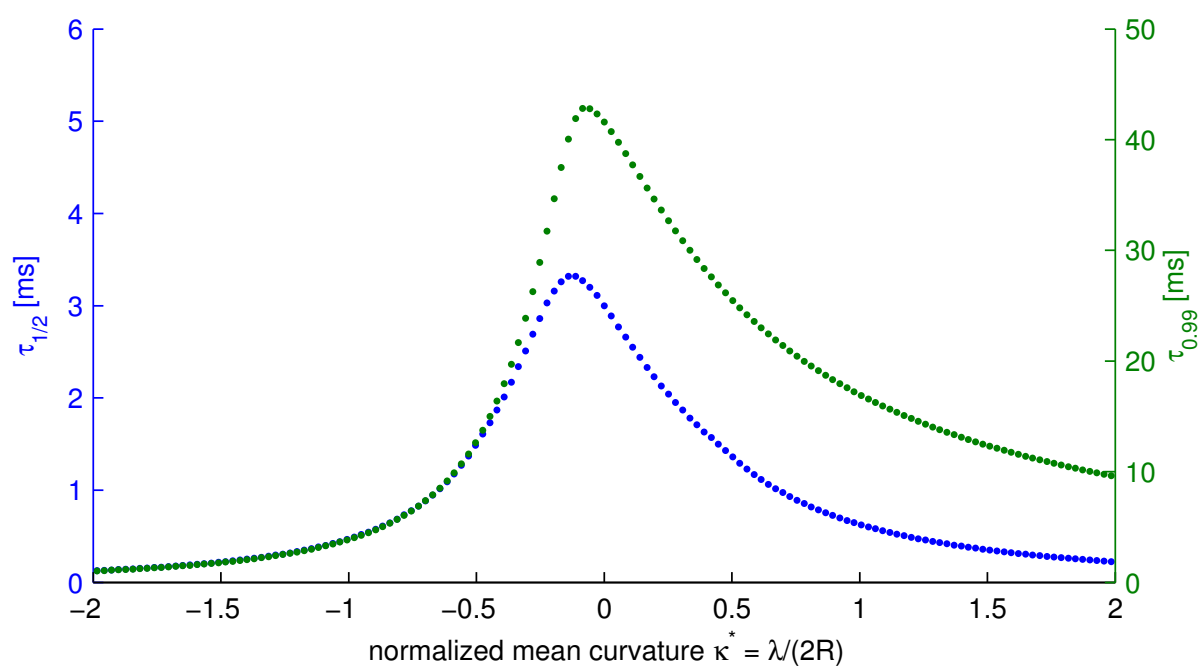

Figure 3.36: Time scale of depolarization at circular boundaries. Data extracted from numerical simulations of the asymptotic case (cf. dots in the plot of figure 3.27). $\tau_{1 / 2}$ and $\tau_{0.99}$ denote the time after which half and $99 \%$ of the asymptotic maximum depolarization of figure 3.27 are reached, respectively, starting from entirely quiescent tissue at the resting membrane potential.

angular pulse shape with the same peak field strength. Here, an exponential decay rate of $\tau_{\text {decr }}=3.33 \mathrm{~ms}$ was used. The mean electric field strength in one half of the biphasic pulse can be calculated as

$$
\begin{aligned}
\bar{E} & =\frac{2}{T}{ }_{\text {pulse }} \int_{0}^{T_{\text {pulse }} / 2} E_{\text {peak }} \exp \left(-t / \tau_{\text {decr }}\right) \mathrm{d} t \\
& =E_{\text {peak }} \frac{2 \tau_{\text {decr }}}{T_{\text {pulse }}}\left[1-\exp \left(-\frac{T_{\text {pulse }}}{2 \tau_{\text {decr }}}\right)\right] .
\end{aligned}
$$

Correcting the data points for the exponential wave form by this factor $(\approx 0.518$ in this case) yields the black triangles in figure 3.35. The corrected data are indeed considerably closer to those of the other pulse shapes. The initial high field strength for each half of the truncated exponential wave form seems to be even advantageous for large $\left|\kappa^{*}\right|$, indicating that the depolarization in this curvature regime takes place on a much smaller time scale, such that the system is able to resolve this specific feature of the exponential wave form. In contrast, for small $\left|\kappa^{*}\right|$, the depolarization time scale is large enough to average out the specific pulse shape.

The relative behavior of $E_{\text {req }}$ for biphasic and monophasic wave forms points into the same direction: The ratio between the two required field strengths, plotted in figure 3.35 $)$, tends towards $\approx 1$ for large positive and negative curvatures and features a maximum at small negative curvatures. Again, a longer time scale for the depolarization process in the small- $\left|\kappa^{*}\right|$ regime would explain this behavior, as it would diminish the overall effect by partial averaging the opposite-polarity halves of the pulse.

To get a more direct picture of the time scales governing the depolarization process at the boundary, the numerical simulations carried out for figure 3.27 (black dots) can be utilized: Figure 3.36 displays the time $\tau_{x}$ necessary to elevate the membrane potential 
from the resting potential to a certain percentage $x$ of the final maximum depolarization. For both values of $x$ (50\% and 99\%), the curves are consistent with the behavior observed above: A maximum at small negative curvatures indicates a slow response of the boundary to the applied electric field, whereas towards large positive and negative curvatures, the maximum depolarization is reached much more rapidly. The overlay of the plots for $\tau_{1 / 2}$ and $\tau_{0.99}$ shows that for large negative curvatures, the two time scales are proportional to each other (indicating a similar relative time course of convergence, independent of the curvature), whereas towards positive curvatures, the depolarization process slows down more and more when approaching the maximum depolarization.

Besides explaining the relative behavior of the required field strengths for different pulse shapes shown in figure 3.35 , the small time scales for high positive and negative curvatures lead to another interesting phenomenon for biphasic pulses: For the two successful activations depicted in figure 3.34 , the action potential is always elicited by the first half of the biphasic pulse. In contrast, for larger positive and negative curvatures, the second (negative) half of the pulse is dominating the dynamics, such that the activation starts on the opposite side of the boundary. The cases for the smallest negative and positive curvatures for which this behavior is observed are depicted in figure 3.37. A possible explanation is that short time scales imply a strong force driving the membrane potential to its final value, which allows the second half of the pulse to suppress an action potential emerging as a result of the positive-polarity part. The asymmetry in the curvatures, for which this effect appears $\left(\kappa^{*}=-0.5\right.$ for negative curvatures; $\kappa^{*}=1.2$ for positive curvatures), is in line with the asymmetry of the ratio between biphasic and monophasic required field strengths (figure $3.35 \mathrm{~b}$ )) and the asymmetry for $\tau_{0.99}$ in figure 3.36 .

In summary, the most important feature of the asymptotic results for circular boundaries is preserved for more realistic scenarios with finite pulse duration and different pulse shapes: Even in full numerical simulations, there is still an optimal size of circular tissue domains in terms of sensitivity. However, the curvature, at which this minimum required field strength is assumed, is shifted somewhat more towards negative curvatures. In addition, a curvature-dependent time scale determines the reaction of the boundary to different pulse shapes. It should be emphasized that the effective time constant for membrane depolarization as depicted in figure 3.36 was extracted from numerical simulations for the asymptotic case (i.e. using subthreshold field strengths). Therefore, the curvaturedependent time scales are not a result of the different field strengths necessary to induce activation and still, as seen above, they can explain some of the features observed for superthreshold stimulation.

\subsubsection{Brief summary}

In section 3.2, the effect of geometry on the membrane potential changes induced by electric fields was investigated. For this purpose, in section 3.2.1, a low-field strength approximation was derived, based on the mono-domain description of cardiac tissue outlined in section 2.1.3. This approximation incorporates the effect of the electric field by a modified boundary condition and corresponds to the ad-hoc theoretical ansatz of PUMIR and KRINSKY et al. [127, 130]. Based on the central equation (3.16), in sections 3.2.3 and 3.2 .4 , general properties relating to electric field orientation, scaling behavior and the 

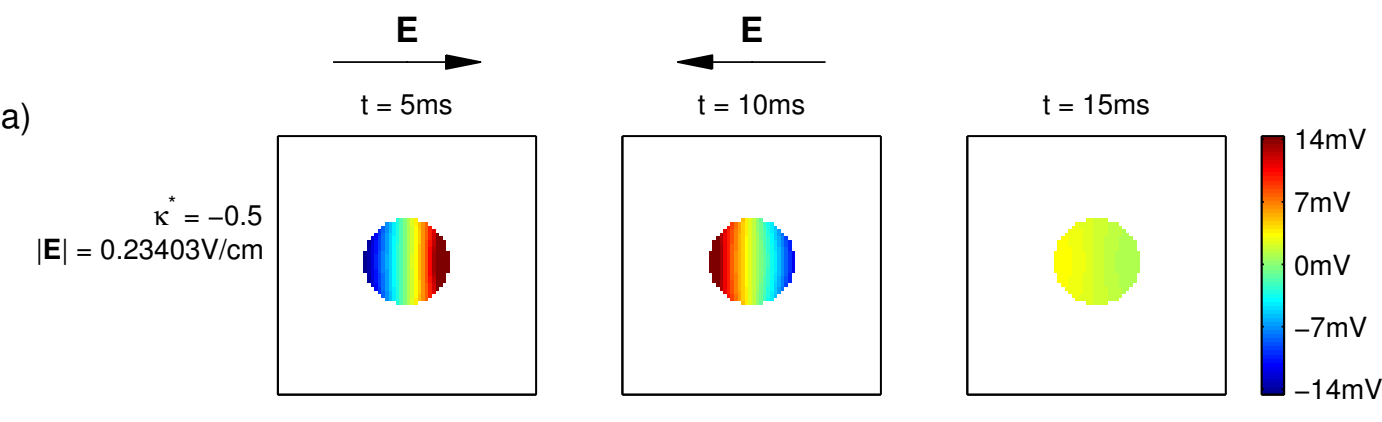

b)
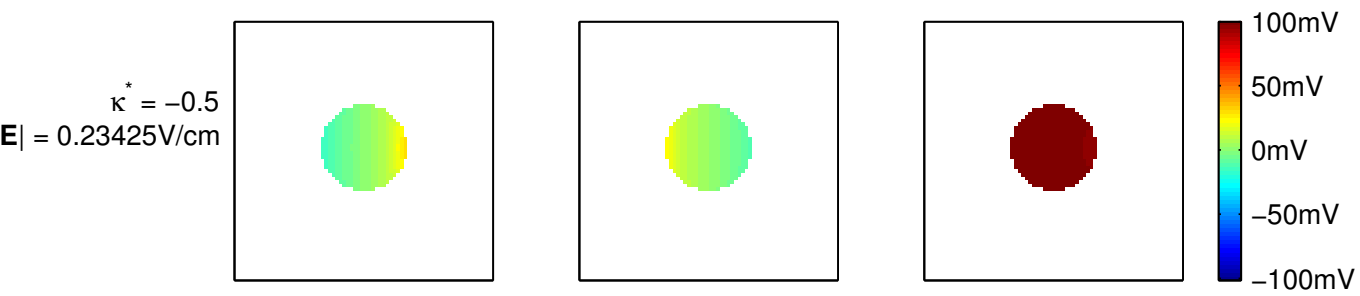

c)
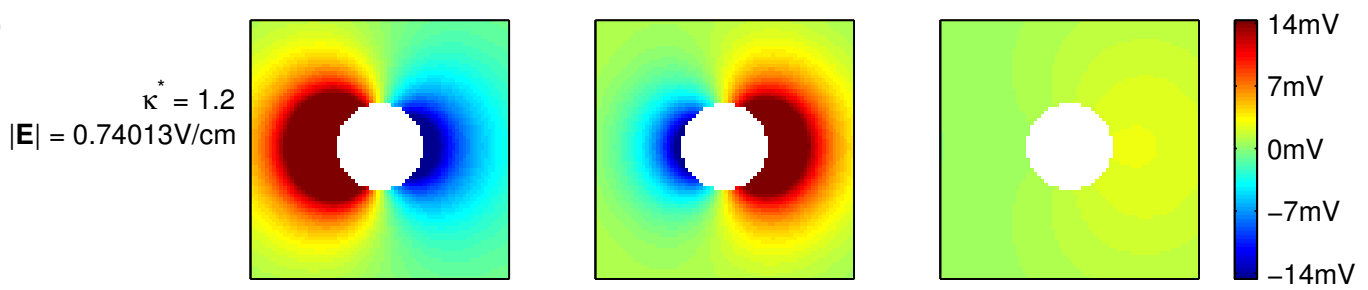

d)
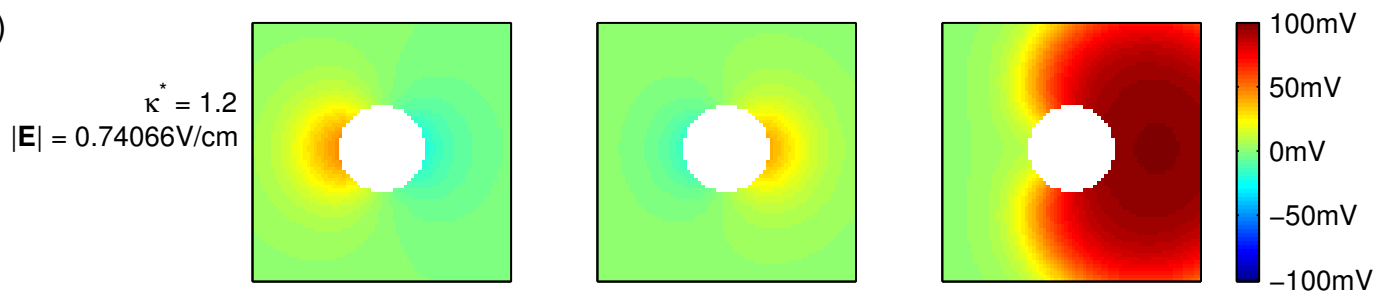

Figure 3.37: Critical field strength for biphasic rectangular pulses: large curvatures. Figure similar to figure 3.34 , but for larger curvatures $\left.\left|\kappa^{*}\right|: \mathbf{a}\right)$, b) Subthreshold and superthreshold stimulation, respectively, of a circular tissue domain of normalized mean curvature $\kappa^{*}=-0.5$. c), d) Subthreshold and superthreshold stimulation, respectively, of a tissue domain containing a circular obstacle and with a normalized mean curvature of $\kappa^{*}=1.2$. Note that, in contrast to figure 3.34 , the tissue is activated by the second half of the biphasic pulse in b) and d). 
dimensionality of the tissue domain were derived. These allow for statements about the sign of membrane potential deflections, the transfer of known results to systems with a different characteristic length scale $\lambda$ and the generalizability of two-dimensional results to three-dimensional structures even without knowing the exact depolarization patterns. The relevance of boundary shape for the effect strength of an electric field pulse is indicated by the cell culture experiments presented in section 3.2.5. Following this indication, the mean curvature $\kappa$ was introduced in section 3.2 .6 as a quantitative measure for boundary shape. Supported by numerical simulations as outlined in section 3.2.2, the depolarization patterns for different kinds of two-dimensional boundaries were determined in sections $3.2 .7,3.2 .8$, 3.2.9 and 3.2.10, revealing effect strengths in the negative-curvature regime, which exceed the hitherto assumed range of possible values. Each of the considered prototypical geometries demonstrated a specific combination of mechanisms which enhance or diminish the maximum depolarization. These mechanisms include the proximity of hyperpolarized boundaries (circular boundaries), the spatial scale of anatomical features (protrusions) and pure curvature effects (parabolic boundaries). All geometries, including the three-dimensional boundaries presented in section 3.2.11, were shown to exhibit a similar behavior for low curvature, as described by equation (3.42). Using numerical simulations with finite field strengths, the relevance of the asymptotic theoretical results for superthreshold stimulation was shown qualitatively on a realistic geometry in section 3.2 .12 and quantitatively for circular boundaries in section 3.2.13. The latter revealed a possible relevance of shape-dependent depolarization time scales for realistic scenarios. For a detailed summary and a discussion of the results, see section 4. 


\subsection{Heterogeneity-induced wave sources in low-energy de- fibrillation 16}

In section 3.2 , the response of cardiac tissue boundaries to electric-field stimulation was investigated theoretically and in numerical simulations. Knowledge about the fundamental mechanisms that govern the initiation of waves by electric fields is required to understand how electrical stimulation influences wave activity that is already present in the tissue. An important field of application for a comprehensive understanding of field-induced wave sources in combination with wave interaction mechanisms is that of anti-arrhythmic pacing strategies, the most prominent among which is defibrillation. The investigations that will be presented in this section have become part of a publication [201], which, on the one hand, demonstrates that LEAP is effective for both ventricular and atrial fibrillation in-vitro as well as atrial fibrillation in-vivo. On the other hand - and that is the contribution of this work - it sheds some new light on the mechanisms that govern wave source recruitment for LEAP, by providing a strong empirical basis for the specific anatomical structures that give rise to wave sources during LEAP.

\subsubsection{Hypothesis}

As indicated in the introduction of this thesis, the tree of coronary arteries and veins is present everywhere in the heart to supply the tissue with oxygen and nutrients, organized in a branching network from the large coronary vessels of millimeters in diameter downto the capillaries with a diameter of a few micrometers. Furthermore, the branching structure of the cardiovascular tree has been shown to follow certain laws that are based on the minimization of construction and fluid conduction cost [225, 226, 227],17] Although the relations derived in these references cannot be transformed directly into a probability distribution of vessel sizes [182], it is reasonable to assume that such statistics is determined by the same fundamental laws and therefore preserved across individuals, guaranteeing the presence of suitable heterogeneities for electric-field stimulation - provided, of course, it has been confirmed in a sufficient number of samples. The anatomical and physiological properties of cardiac vessels thus suggest the hypothesis that they might be the primary source of heterogeneity for LEAP. Then, of course, the question of whether and how the mechanisms involved could be accessed experimentally becomes inevitable.

How could the relevance of the tree-like vessel system for LEAP be tested? Given that fibrillation itself is a turbulent and complex activation pattern, analyzing the interactions of field-induced waves with this activity is a challenging task. There is an even more obstructive technical problem: the cardiac muscle is a three-dimensional system, but the imaging technique described in section 2.3 .2 can only collect information from the surface of the muscle, rendering it impossible to directly observe waves in the bulk of tissue. Nevertheless, some information about intramural activity can be inferred from observations of the surface (e.g., the direction of wave fronts [229]) and there are efforts to create suitable three-dimensional imaging methods using optical coherence tomography

\footnotetext{
${ }^{16}$ The results obtained in adult beagle dogs which are presented in this section have been published in reference 201.

${ }^{17}$ See also reference 228 and references therein.
} 
[230], transillumination tomography [231] or ultrasound imaging (via contraction) [232]. However, resolution and/or penetration depth are too limited to observe a large volume of tissue in real-time and to simultaneously detect anatomical structures such as blood vessels.

Therefore, an alternative approach is chosen here, using the following procedural simplifications:

- Instead of trying to directly observe the interaction of induced waves with fibrillation during LEAP, waves are observed in quiescent tissue. This is to separate the mechanisms of wave initiation from the interaction with complicated activity. Consequently, one has to bear in mind that the wave sources considered from now on are actually only potential wave emitting sites and their recruitment in a LEAP setting depends on the dynamics already present in the medium.

- Due to the unavailability of adequate three-dimensional high-resolution imaging methods, a model for the activation of blood vessels by electric fields is constructed to predict a quantity that is observable by optical mapping techniques: the activation time $\tau$ (see section 2.3.4).

- Structural information required for the above model is not extracted during the optical mapping experiment, but using a suitable tomography technique such as $\mu \mathrm{CT}$ with a contrast agent injected into the coronary vessel tree. This way, the data can be obtained with high spatial resolution, sacrificing, however, a direct correlation of observed waves and locations of blood vessels.

\subsubsection{Theoretical framework}

The simple model that is to be constructed is based on the following fundamental assumptions:

1. Heterogeneities are discrete, circular obstacles with a radius $R$. When electrically stimulated, a heterogeneity either is recruited as a wave source or it remains silent (does not emit a wave), depending on the field strength of the pulse.

2. Heterogeneities are distributed homogeneously throughout the myocardium.

3. Once recruited as a wave emitting site, the heterogeneity acts as a point source and the excitation wave spreads outwards with a constant velocity.

Using assumption 3 , a single wave source activates a volume

$$
V_{1}(t)=\gamma(v t)^{d} \quad \text { with } \gamma= \begin{cases}\pi & d=2 \\ \frac{4}{3} \pi & d=3\end{cases}
$$

of the tissue, where $t$ is the time after the stimulation and $v$ is the conduction velocity. $d$ is the dimensionality of the tissue, which can be either viewed as a two-dimensional sheet or a three-dimensional volume. In reality, the anisotropy of cardiac tissue (see section 1.3) leads to an ellipsoidal rather than spherical wave propagation. However, as will be argued below, $\gamma$ and $v$ will be consumed into an arbitrary constant and only the scaling $V_{1} \propto t^{d}$ 
is relevant, which also holds for ellipsoidal wave propagation.

Ignoring the overlap between the waves emitted from different sources and employing assumption 2, the activation time of a given volume $V$ is the time $\tau$, for which

$$
V=N_{s} V_{1}(\tau)=N_{s}(E) \gamma(v \tau)^{d}
$$

holds, when $N_{s}(E)$ is the field-strength dependent number of wave sources. Let $\rho(E)=$ $N_{s}(E) / V$ be the wave source number density, then the activation time $\tau$ can be calculated as

$$
\tau(E)=v^{-1}[\gamma \rho(E)]^{-\frac{1}{d}} .
$$

To turn this equation into a prediction for $\tau, \rho(E)$ has to be estimated theoretically: It is assumed that blood vessels act like a tissue boundary, meaning that the intracellular space of the electrophysiologically active substrate ends abruptly at the surface of the blood vessel (i.e. is insulated), and the extracellular space is continuous. This corresponds to the assumptions that lead to the theory presented in section 3.2 . The tubular vessels are modeled locally as cylinders. Using the results of section 3.2 , or, more specifically, the dimensionality equivalence explained in section 3.2.4, this implies that the theoretical results for membrane potential changes at two-dimensional circular boundaries can be applied to these three-dimensional structures. The theory for circular obstacles was already developed in references [127, 130] and corresponds to the positive-curvature part of figure 3.27 (page 127). Therefore, assuming that the electric field is correctly oriented, the maximum depolarization depends on the radius $R$ of the blood vessel and is given by equation (3.35):

$$
e^{\max }(R)=-\lambda|\mathbf{E}| \frac{K_{1}(R / \lambda)}{K_{1}^{\prime}(R / \lambda)}
$$

According to sections 3.2 .12 and 3.2 .13 , the dominating factor that determines whether a tissue boundary emits a wave is indeed the maximum depolarization. Let $e_{t}$ be the excitation threshold for a cardiac cell, i.e. the deviation from the resting membrane potential which just elicits an action potential. Then

$$
e^{\max }(R) \geq e_{t}
$$

is the condition for a blood vessel to be recruited as a wave emitting site. The positivecurvature regime of figure 3.27 relevant for blood vessels shows that equation (3.57) is monotonically decreasing with the curvature $\kappa=1 /(2 R)$, i.e. monotonically increasing with $R$. This means that the condition 3.58 is fulfilled automatically for all $R>\hat{R}$, if it is fulfilled for $\hat{R}$. Conversely, for any given $\lambda$ and $|\mathbf{E}|$, there is a minimum radius $R_{\min }$, such that equation (3.58) is fulfilled if and only if $R>R_{\min }$ and $R_{\min }$ is the value of $R$ for which equation (3.58) reaches equality. Substituting equation (3.57), $R_{\min }$ is calculated as

$$
R_{\min }(E)=\lambda\left[\frac{K_{1}^{\prime}}{K_{1}}\right]^{-1}\left(-\frac{\lambda E}{e_{t}}\right)
$$


where $\left[K_{1}^{\prime} / K_{1}\right]^{-1}$ is the inverse function of $K_{1}^{\prime} / K_{1}$ and $E=|\mathbf{E}|$. Equivalently, $E_{\text {req }}\left(R_{\text {min }}\right)$ is the field strength required to excite blood vessels with a radius larger than $R_{\min }$ :

$$
E_{\mathrm{req}}\left(R_{\mathrm{min}}\right)=-\frac{e_{t}}{\lambda} \frac{K_{1}^{\prime}\left(R_{\min } / \lambda\right)}{K_{1}\left(R_{\min } / \lambda\right)}
$$

The sizes of blood vessels in the tissue are extracted from $\mu \mathrm{CT}$ scans. The result of the segmentation procedure is a list of blood vessel radii, each of which characterizes a blood vessel segment of approximately equal length (along the cylinder axis). The list of vessel radii is used to calculate a probability density function $(\mathrm{PDF}) p(R)$ for the radius of an arbitrarily chosen vessel segment, which is then reinterpreted - according to assumptions 1 and 2 - as a parameter distribution of homogeneously distributed, point-like objects which emit waves if their parameter $R$ fulfills the condition (3.58).

For the smallest blood vessels, arterioles and venules, assumption 2 can be expected to be correct, due to nutrients and oxygen supply constraints and the large number of such structures. Since the vessels are organized in a fractal tree, homogeneity is inherited by larger blood vessels from which the smaller ones branch off. However, it is clear that this kind of inheritance has certain limits: On the other end of the size distribution, the largest coronary vessels each supply one area of the myocardium and their location is strongly preserved across individuals. In between these two extremes, there must be a gradual transition from (smaller) sizes, for which assumption 2 is still approximately fulfilled, to (larger) vessels, for which this cannot be true. Similarly, assumption 1 is fulfilled for the large number of small vessel segments distributed across the entire myocardium, but for the few large vessels, their tubular shape will become important. Therefore, large deviations of the model from reality can be expected primarily for large blood vessels, corresponding to low field strengths $E$.

Once one accepts these limitations of the reinterpretation of $p(R)$, the number of wave sources $N_{s}(E)$ can be calculated as

$$
N_{s}(E)=N_{s}^{\max } \int_{R_{\min }(E)}^{\infty} p(R) \mathrm{d} R .
$$

$N_{s}^{\max }$ denotes the maximum number of wave sources recruited if the whole range of sizes characterized by $p(R)$ is activated (i.e. for $R_{\min }(E)=0$ ). Substituting equation (3.61) into equation 3.56 leads to the following expression:

$$
\tau(E)=v^{-1}\left[\gamma \frac{N_{s}^{\max }}{V} \int_{R_{\min }(E)}^{\infty} p(R) \mathrm{d} R\right]^{-\frac{1}{d}} .
$$

Together with equation (3.59), this formula provides a link between the activation time $\tau$ (observable by optical imaging) and $p(R)$, which encodes purely anatomical information about the cardiovasculature. The conceptual basis of this link is illustrated again in figure 3.38 . In fact, the activation time can only be predicted up to an unknown factor: The prefactor $v^{-1}\left(\gamma N_{s}^{\max } / V\right)^{-1 / d}$ consists of a number of peparation-specific constants. In particular, $N_{s}^{\max }$ is a rather abstract quantity originating from the reinterpretation 


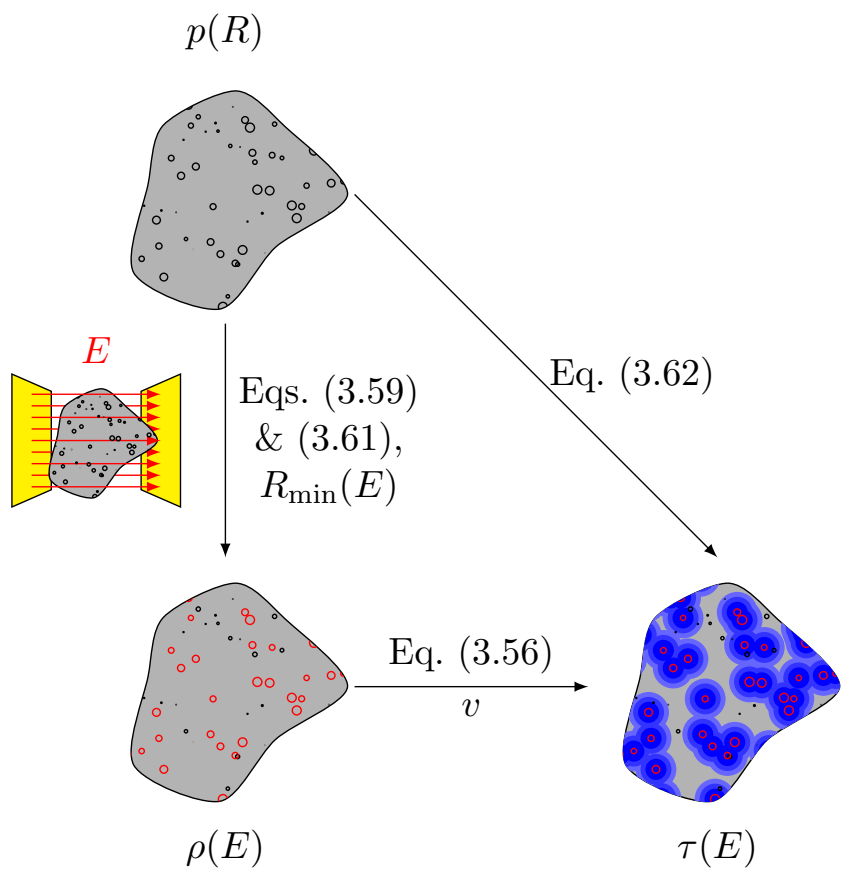

Figure 3.38: Wave source recruitment model. The tissue is assumed to contain obstacles (top left; black circles) of all radii $R$ with a PDF $p(R)$. For each field strength $E$, theory predicts a certain minimum size $R_{\min }(E)$ above which all obstacles are recruited as wave emitting sites (lower left; red circles) and which results in a certain wave source density $\rho(E)$. The activation time $\tau$ is then estimated by assuming simultaneous radial wave spread from these wave sources (lower right; activation shown in blue).

of the size distribution $p(R)$. As this number cannot be determined experimentally, the prefactor as a whole can be redefined as an unknown proportionality constant $\xi$, such that the equation reads

$$
\tau(E)=\xi\left[\int_{R_{\min }(E)}^{\infty} p(R) \mathrm{d} R\right]^{-\frac{1}{d}}
$$

Assuming the size distribution follows a power law, $p(R)=c R^{\alpha}$ with $\alpha<-1$, a result for the scaling of $\tau(E)$ independent of the prefactor $\xi$ can be obtained in the limit of large field strengths: if $\lambda E / e_{t} \gg 1$, it follows that $R \ll \lambda$. In this case, the small-obstacle approximation of equation (3.47) (page 129 ) holds and $R_{\min }(E) \approx e_{t} / E$. Substituting this into equation 3.62 yields

$$
\tau(E)=\xi\left[\int_{R_{\min }(E)}^{\infty} c R^{\alpha} \mathrm{d} R\right]^{-\frac{1}{d}}=\xi\left(-\frac{c}{\alpha+1} R_{\min }(E)^{\alpha+1}\right)^{-1 / d}=\tilde{\xi} E^{(\alpha+1) / d}
$$

In the last step, all factors were, again, summarized in a proportionality constant $\tilde{\xi}>0$. The result implies that, for large field strengths $E$, a power law for $p(R)$ with an exponent 
$\alpha$ leads to a power law for $\tau(E)$ with an exponent $\beta=(\alpha+1) / d$ :

$$
p(R)=R^{\alpha} \quad \stackrel{\beta=(\alpha+1) / d}{\longleftrightarrow} \quad \tau(E)=E^{\beta} .
$$

So far, the main focus was on predicting an observable dynamic effect from structural data. One can also ask the converse question: is it possible to learn something about the size distribution of heterogeneities from the dynamical response of the tissue? Indeed, equation $(3.63)$ can also be read backwards: given the measured activation time as a function of $\tau(E), p(R)$ could be calculated by differentiation with respect to $E$. Since $\tau(E)$ is measured in experiments only at discrete field strengths $E$ and with a certain degree of inaccuracy, differentiating $\tau(E)$ is impractical. However, the integral of $p(R)$ within certain bins of radii can be determined directly from measured $\tau(E)$ values by subtracting equation (3.63) for two different field strengths.

$$
\int_{R_{\min }\left(E_{i+1}\right)}^{R_{\min }\left(E_{i}\right)} p(R) \mathrm{d} R=\xi^{d}\left(\tau\left(E_{i+1}\right)^{-d}-\tau\left(E_{i}\right)^{-d}\right)
$$

$E_{i+1}>E_{i}$ are the field strengths for which $\tau$ was measured. Because of the monotonicity of $R_{\min }$, this implies that $R_{\min }\left(E_{i+1}\right)<R_{\min }\left(E_{i}\right)$.

\subsubsection{Blood vessel size distributions}

Size distributions $p(R)$ were obtained separately for atria and ventricles. Contrast agent (MicroFil, Flow Tech Inc., USA) was injected into the coronary arteries and the sample was scanned at the $\mu \mathrm{CT}$ Facility for Imaging and Preclinical Research/Cornell University by Mark L. Riccio. The resulting voxel data was analyzed by Daniel Hornung using a custom-made segmentation algorithm which is outlined in the Supplementary Material of reference [201] and described in detail in reference [182]. Briefly, a skeleton of the vascular tree approximating the center lines of the arteries was obtained by applying an intensity threshold and using a skeletonization algorithm. Then, the radius of the vessels at each point on the skeleton was estimated by fitting a plateau function to the intensity profile of the $\mu \mathrm{CT}$ data in a plane perpendicular to the center line. Histograms of the detected radii $R_{i}, i=1 \ldots N_{R}$ for exemplary ventricular and atrial preparations are shown in figure $3.39 \mathrm{a}$ ) and b). Because of the rapid decrease of size frequencies towards larger vessel sizes, logarithmically spaced bin edges $\hat{R}_{0}>\hat{R}_{1}>\hat{R}_{2}>\ldots$ (shown as vertical dashed lines in figure 3.39 are chosen to produce an approximation of the PDF $p(R)$ :

$$
p_{j}=\frac{1}{N_{R}\left(\hat{R}_{j-1}-\hat{R}_{j}\right)} \sum_{\substack{i \\ \hat{R}_{j} \leq R_{i} \leq \hat{R}_{j-1}}} 1 \quad \forall j>1
$$

The $p_{j}$ can be interpreted as an approximation to $p(R)$ at the geometric center $\sqrt{\hat{R}_{j} \hat{R}_{j-1}}$ of the bin (e.g., for fitting purposes). These approximations are shown in figure 3.39 r) and d). They show, in the limited range of sizes where reliable segmentation was possible, a behavior compatible with a power law.

In addition, the $p_{j}$ are correspond directly to the integrals of equations (3.63) and (3.66) 

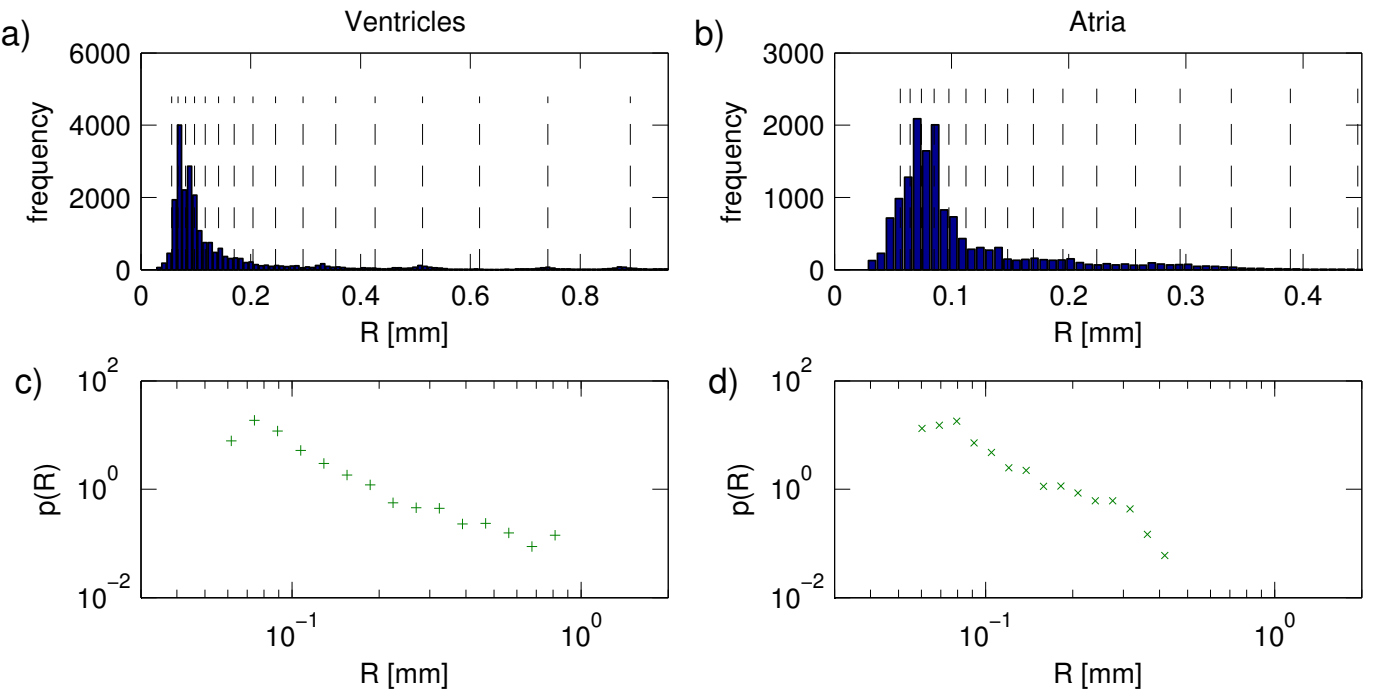

Figure 3.39: Size distributions of blood vessels. a), b) Histograms of ventricular and atrial detected blood vessel radii in canine hearts. Prominent features are the rapid decrease of radius counts towards larger vessel sizes. The decrease below approximately $70 \mu \mathrm{m}$ is due to the resolution limit of the $\mu \mathrm{CT}$ data $(50 \mu \mathrm{m})$. Vertical dashed lines indicate logarithmic bins. c), d) Log-log plots of PDFs for the radius distributions shown in a) and $\mathrm{b}$ ), respectively, calculated using the indicated bins. The values of $p(R)$ for each bin are plotted at the $x$-coordinate corresponding to the geometric center of the bin.

via the relations

$$
\begin{gathered}
\int_{R_{\min }\left(E_{i}\right)}^{\infty} p(R) \mathrm{d} R=\sum_{k=1}^{i} p_{k}\left(\hat{R}_{k-1}-\hat{R}_{k}\right) \\
\int_{R_{\min }\left(E_{i}\right)}^{R_{\min }\left(E_{i-1}\right)} p(R) \mathrm{d} R=p_{i}\left(\hat{R}_{i-1}-\hat{R}_{i}\right),
\end{gathered}
$$

provided that $E_{i}$ and $\hat{R}_{i}$ are related by equation 3.59 or equation 3.60), i.e. $\hat{R}_{i}=$ $R_{\min }\left(E_{i}\right)$ or $E_{i}=E_{\text {req }}\left(\hat{R}_{i}\right)$. Thus, the bin edges for $p(R)$ determine the points for which a predicted $\tau(E)$ can be calculated, and conversely, the values of $E$ for which $\tau(E)$ is measured determine intervals for which the size distribution of heterogeneieties can be estimated from optical mapping data.

\subsubsection{Activation times}

Activation times were obtained according to the procedure described in section 2.3.4. Typical activation patterns observed in an isolated canine atrium are shown in figure 3.40. The activation time dependence resulting from multiple measurements in a ventricular and an atrial tissue preparation can be found in figure 3.41. In particular for the atrial preparation in figure $3.41 \mathrm{~b}$ ) and $\mathrm{d}$ ), it is evident that there is a large variability of the activation times at low field strengths. In this regime, only very few heterogeneities or tissue boundaries are recruited as wave emitting sites, such that the activation time is very sensitive to changes in the number of wave sources. Consequently, the slightest change 


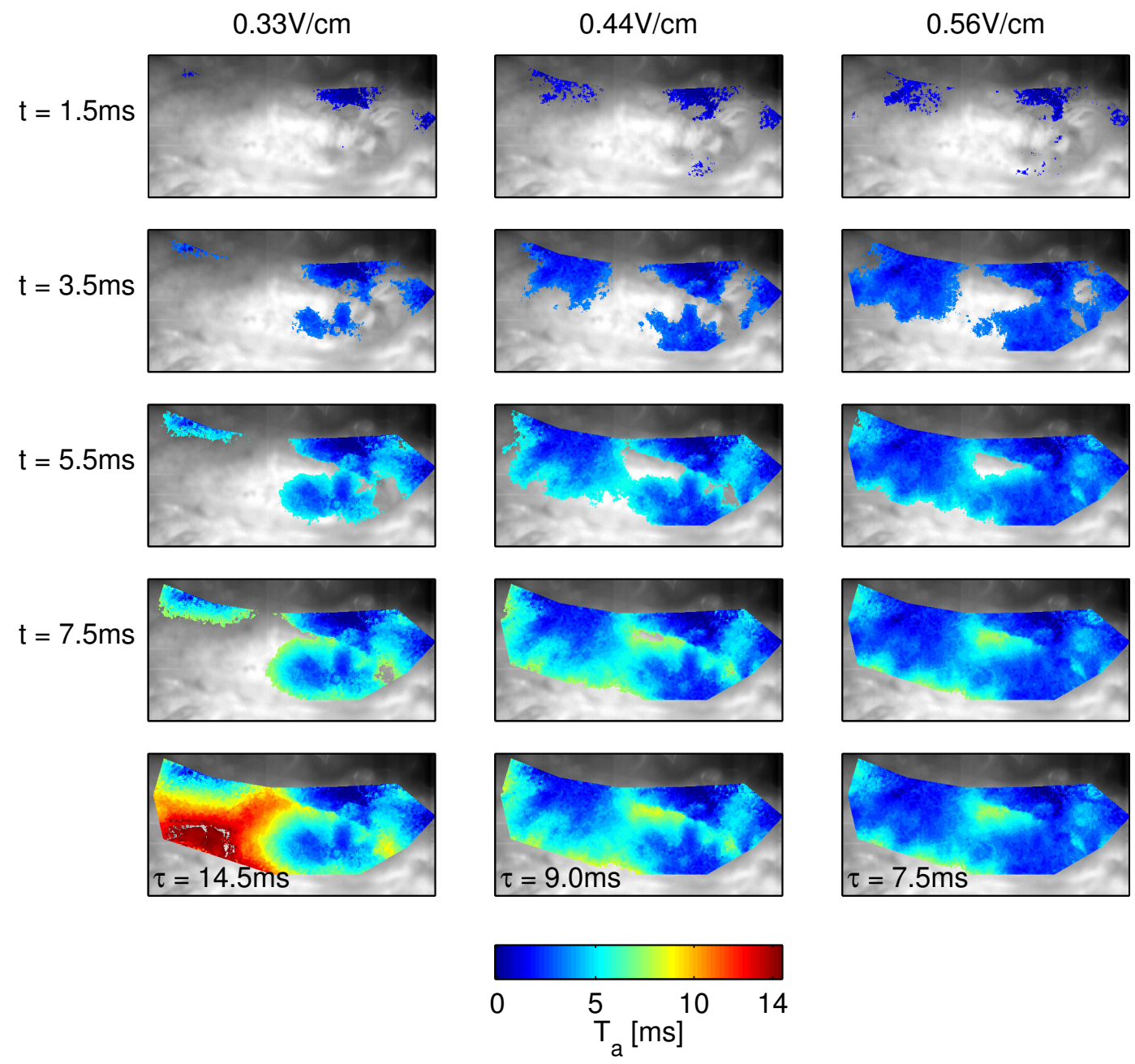

Figure 3.40: Tissue activation for different field strengths. Activation patterns of the same tissue preparation (canine dog, atrium) for three different field strengths (see column headings). The color code is identical for all cases, the total activation time $\tau$ is denoted in the bottom row. Activation maps show both faster activation and increased number of breakthrough sites for higher field strengths, the latter corresponding to minima in the activation map. 
a)

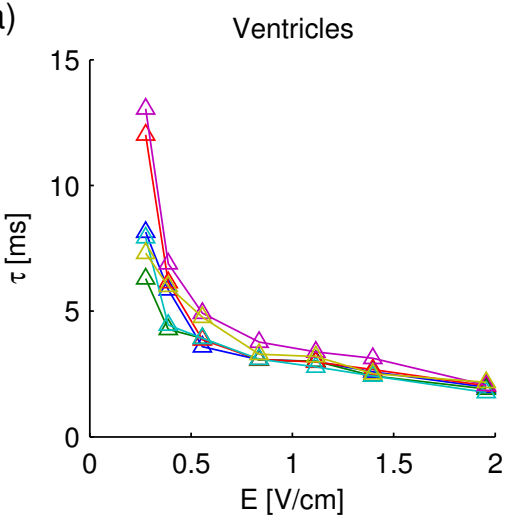

C)

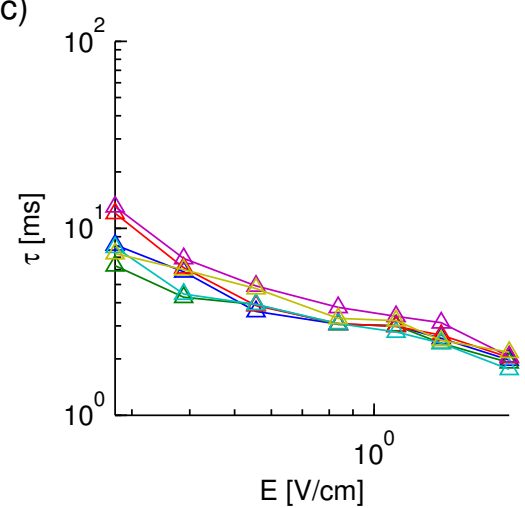

b)

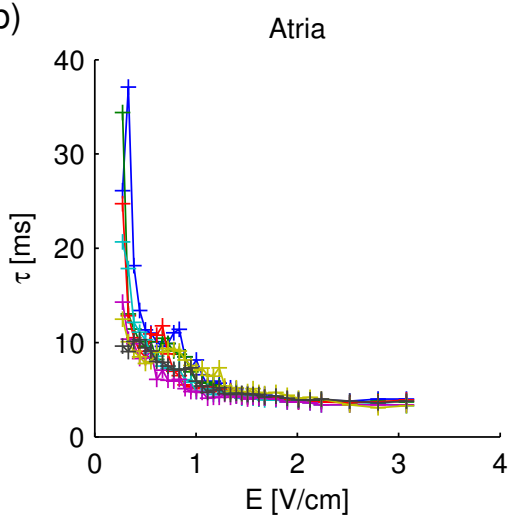

d)

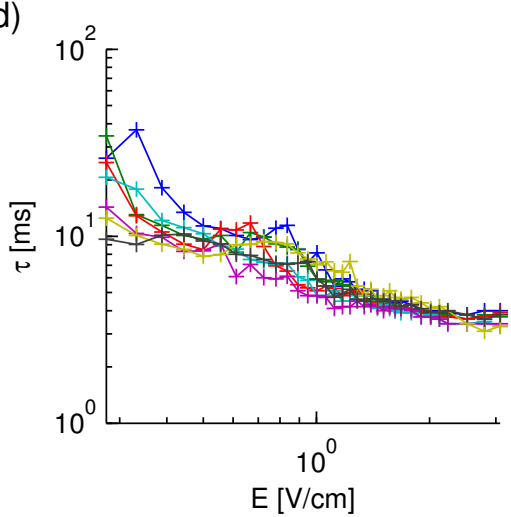

Figure 3.41: Observed activation times. Multiple measurements of the activation time $\tau(E)$ in a single preparation of $\mathbf{a}$ ) ventricular tissue and $\mathbf{b}$ ) atrial tissue of canine hearts. c), d) Log-log plots of the same data as in a) and b), respectively. 
a)

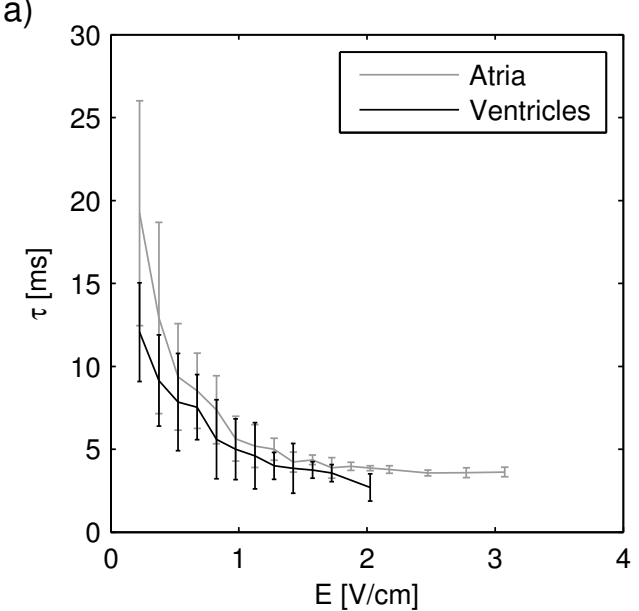

b)

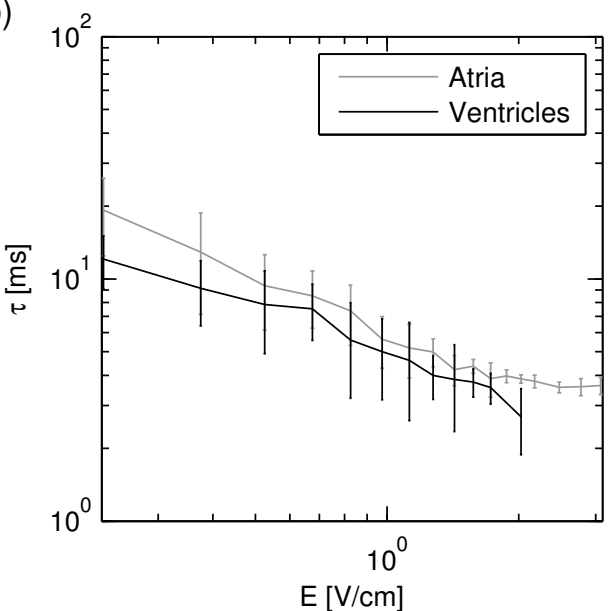

Figure 3.42: Averaged activation times. Activation times binned and averaged from all atrial ( $N=3,17$ measurements) and ventricular $(N=5,22$ measurements) preparations of canine hearts. a) Activation time $\tau$ versus field strength $E$. At high field strengths, atrial activation times start to saturate at about $3 \mathrm{~ms}$ due to the limited camera time resolution. b) Log-log plot of the same data. Error bars indicate standard deviations across different measurements.

of physiological and/or dynamical conditions in the time between two measurements with the same field strength can drastically change the activation time. Even if the behavior is less volatile at low field strengths, as for the ventricular prep in figure 3.41 ) and c), it can be expected that the activation time will vary greatly across different samples. To produce an accurate picture of the general behavior of the activation time, all measurements are averaged in predefined bins. As discussed in section 3.3.3, the positions of the bins determine, at the same time, for which radii the PDF integrals of $p(R)$ can be calculated. One has to keep in mind that averaging activation times from different preparations involves another systematic error: for very low source densities (i.e., low field strengths), the activation time for a specific prep is not only a function of the source density, although the central model equation (3.56) suggests that this be the case. In the extreme situation where only a single wave emitting site is created by the electric-field pulse, the measured time from the pulse to complete activation depends on the maximum distance from the source of any point in the field of view of the camera. Even the activation time as defined in equation (2.103), although being independent of the time of the pulse, still depends on the dimensions of the observed part of the tissue. Relics of this dependence disappear only gradually towards higher field strengths, when there is a large number of wave sources not only in the whole tissue, but in the field of view, and the homogeneity assumption 2 (page 145 is fulfilled more accurately. As this kind of systematic error depends on the concrete spatial arrangement of wave sources in a given tissue sample and its magnitude depends on field strength, there is no simple normalization procedure to correct for it. Therefore, the averaging procedure is carried out with the original, unmodified activation times, even from different tissue preparations (see figures B.1 and B.1 in the appendix for all individual data sets). The resulting mean curves are shown in figure 3.42 . The error bars at low field strengths therefore not only represent a measurement error, but also include the aforementioned systematic error stemming from the choice of the field of view and natural 


\begin{tabular}{rcc} 
& $\hat{R}_{i}$ & $E_{i}$ \\
\hline \hline atria & $10^{0.97-i \cdot 0.06} \mathrm{~mm}$ & $i \cdot 0.15 \mathrm{~V} / \mathrm{cm}$ \\
ventricles & $10^{0.99-i \cdot 0.08} \mathrm{~mm}$ & $i \cdot 0.15 \mathrm{~V} / \mathrm{cm}$
\end{tabular}

Table 3.3: Summary of binning parameters. Bin edges used for calculating $p(R)$ as introduced in section 3.3 .3 and for averaging activation times as discussed in section 3.3 .4 .

variability in wave source arrangement.

\subsubsection{Linking structure and function}

In total, $\mu \mathrm{CT}$ data from three ventricular and five atrial tissue preparations was available. The size distributions $p(R)$ (or rather the $p_{j}$ as introduced in section 3.3.3) are plotted in figure $3.43 \mathrm{a}$ ) and figure $3.44 \mathrm{a}$ ). The binning parameters used to obtain an approximation of $p(R)$ from the list of detected vessel radii can be found in table 3.3 . The size distributions were analyzed separately by DANIEL Hornung (see Supplementary Material of [201] and [182]) to have an average scaling exponent of $\alpha=-2.75 \pm 0.30$ for the ventricles and $\alpha=2.74 \pm 0.05$ for the atria. This average scaling behavior is indicated by black lines in figure 3.43 a) and figure 3.44 a). All size distributions are converted separately into wave source densities and activation times using equations (3.61) and (3.56) in conjunction with equation (3.68) ${ }^{18}$ For equation (3.61),$N_{s}^{\max }$ was chosen arbitrarily to produce wave source densities on the same order of magnitude as those calculated from the activation time measurements. Note, however, that this does not change the scaling behavior of the prediction. Otherwise, parameters were kept fixed at physiologically sensible values: $\lambda=1 \mathrm{~mm}$ [233] and $e_{t}=15 \mathrm{mV}$. Technically, a conduction velocity $v=37 \mathrm{~cm} / \mathrm{s}$ was used to convert source densities $\rho$ into activation times $\tau$ and vice versa. However, the conduction velocity is not critical to the overall link between $p(R)$ and $\tau(E)$, as any change can always be compensated by an appropriately chosen $N_{s}^{\max }$, as indicated by the joint proportionality constant $\xi$ in equation (3.63). For the atria as a thin, sheet-like medium, $d=2$ was used, as opposed to $d=3$ for the thick ventricular walls.

The independent predictions of $\rho(E)$ and $\tau(E)$ are then averaged and the standard deviation of their values is used to produce an error estimate at each field strength. The resulting mean predictions are plotted as green diamonds with corresponding error bars in figure 3.43k) and d) (for the ventricles) and figure 3.44 ) and d) (for the atria). Fitting a power law to each of the individual predictions leads to a mean predicted scaling exponent $\beta=-0.74 \pm 0.25$ for the ventricles and $\beta=-0.81 \pm 0.07$ for the atria.

Mean measured activation times and data calculated from them using equation (3.56) are shown as blue squares throughout figures 3.43 and 3.44 . In order to not only obtain an overall scaling exponent for the observed activation time but also a measure for its variability, scaling exponents were determined for each measured activation time curve separately (cf. tables B.1 and B.1 in the appendix). Their mean values are $\beta=-0.73 \pm 0.17$ for the ventricles and $\beta=-0.81 \pm 0.24$ for the atria. Therefore, the prediction of activation times from size distributions yield a scaling behavior that is in excellent agreement with the

\footnotetext{
${ }^{18}$ One bin containing all sizes of blood vessels beyond the upper limit of the plotting range in figures 3.44 ) and 3.43 a) was included in the prediction. This corresponds to blood that are large enough to be activated already at the lowest predicted field strengths.
} 
a)

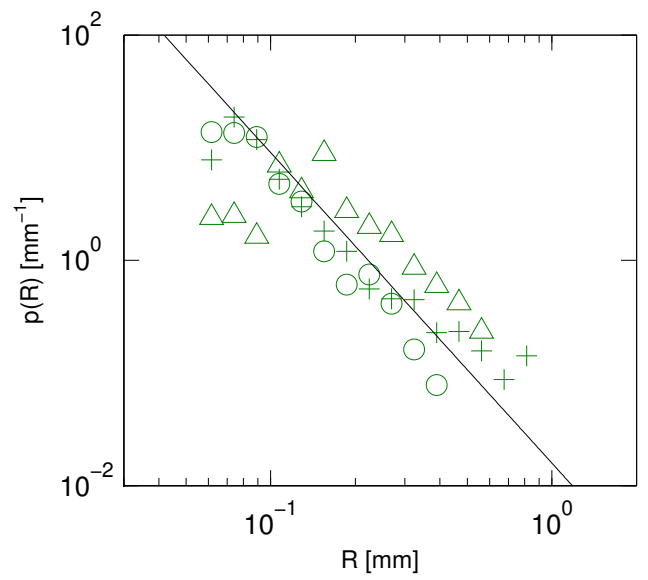

c)

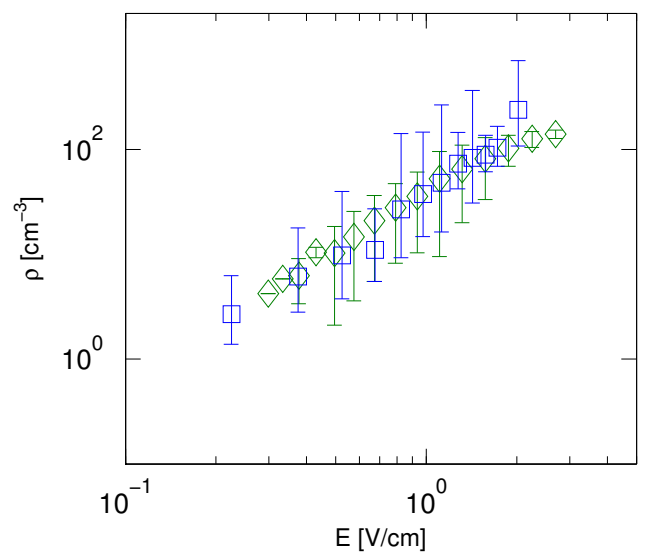

b)

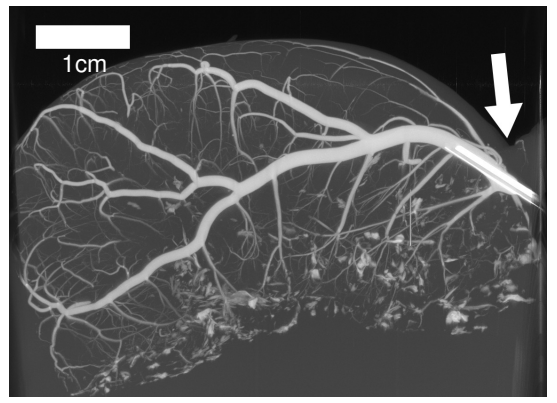

d)

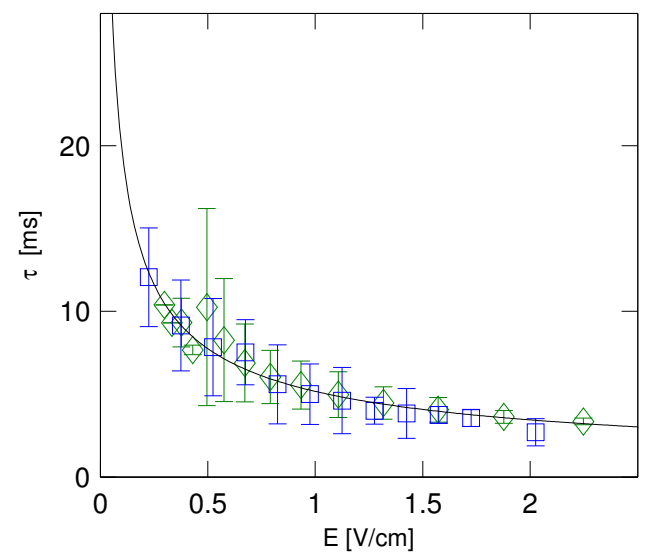

Figure 3.43: Structure vs. function in canine ventricles. a) Size distributions $p(R)$ of blood vessels for three canine ventricular preparations, obtained as described in section 3.3.3. Symbols $+, \circ, \Delta$ indicate different preparations. The black line indicates the mean scaling behavior $p(R) \propto R^{-2.75 \pm 0.30}$ of all preparations. b) Projection of examplary $\mu \mathrm{CT}$ data. The arrow indicates contrast agent injection site. c) Average of wave source density predictions $\rho(E)$ from the three size distributions using equation (3.61) (green diamonds) and a calculation from the average ventricular activation time $\tau(E)$ shown in d) using equation (3.56) (blue squares). d) Average measured activation time $\tau(E)$ in all ventricular preparations (blue squares) and average of activation time predictions from the three size distributions by virtue of equation (3.63) (green diamonds). The black line indicates the power law $\tau \propto E^{-0.58 \pm 0.10}$ obtained from a direct conversion of the exponents as suggested by equation (3.65). Figure adapted from figure 3 in reference [201]. 
a)

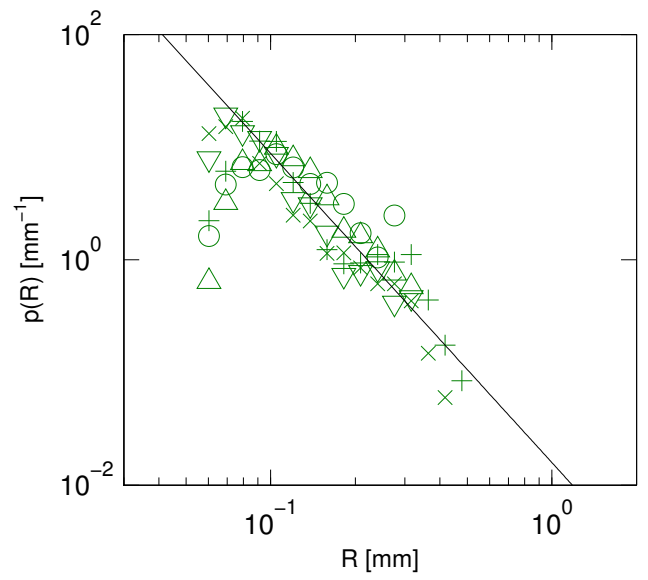

c)

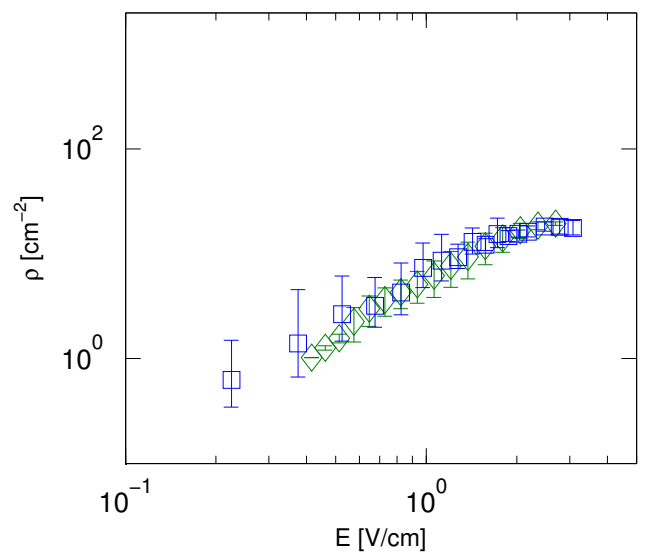

b)

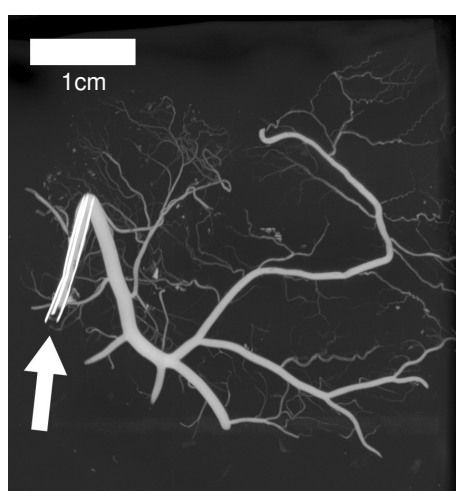

d)

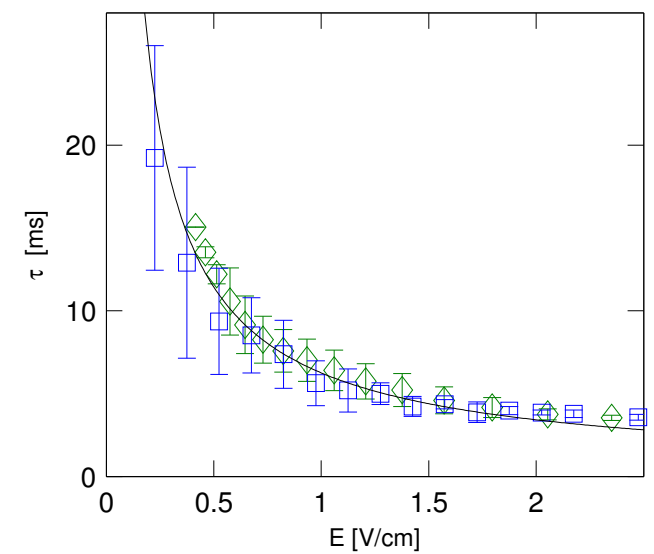

Figure 3.44: Structure vs. function in canine atria. a) Size distributions $p(R)$ of blood vessels for five canine atrial preparations, obtained as described in section 3.3.3. Symbols $+, \circ, \Delta, \times, \nabla$ indicate different preparations. The black line indicates the mean scaling behavior $p(R) \propto R^{-2.74 \pm 0.05}$ of all preparations. b) Projection of examplary $\mu \mathrm{CT}$ data. b) Projection of examplary $\mu \mathrm{CT}$ data. The arrow indicates contrast agent injection site. c) Average of wave source density predictions $\rho(E)$ from the five size distributions using equation (3.61) (green diamonds) and a calculation from the average atrial activation time $\tau(E)$ shown in d) using equation (3.56) (blue squares). d) Average measured activation time $\tau(E)$ in all atrial preparations (blue squares) and average of activation time predictions from the three size distributions by virtue of equation (3.63) (green diamonds). The black line indicates the power law $\tau \propto E^{-0.87 \pm 0.03}$ obtained from a direct conversion of the exponents as suggested by equation (3.65). Figure adapted from figure 3 in reference [201]. 
observed data. In addition, figures 3.43 and 3.44 show that the use of physiological values for the parameters of the model $\left(e_{t}, \lambda\right)$ leads to variations of the activation time in the observed field strength range and that there is good quantitative agreement between the observed dynamical behavior and that expected on the basis of the structural information. Direct conversion of the structural scaling exponents to those for the activation time as suggested by equation (3.65) yields $\beta=-0.58 \pm 0.10$ for the ventricles and $\beta=-0.87 \pm 0.03$ for the atria. Although these exponents are still compatible with the observed ones within error bars, the predictions, in particular for the ventricles, are clearly further away from the observed values. This could simply be a result of the relatively few and different ventricular structures (cf. figure 3.43 )) that were available. However, it could also be evidence for the superiority of the numerical prediction of activation times using equation (3.63) instead of working with scaling exponent averages and an approximate conversion.

Using equation (3.66) together with equation 3.69 , the source densities $\rho(E)$ calculated from the activation time data can further be converted into expected size distributions of the heterogeneities involved. The proportionality constant of equation (3.66) was calculated by imposing $\int p(R) \mathrm{d} R=1$ (which is merely an arbitrary normalization because there can also be heterogeneities with a critical field strength outside the observed activation time range). The result of this calculation is shown in figure 3.45 . As the average activation time curve for the atria is not monotonic, no meaningful size distribution can be calculated for the corresponding radius intervals, as the model assumes that higher field strengths can only recruit additional wave sources, not fewer. These points are omitted in figure $3.45 \mathrm{~b}$ ). Nevertheless, the calculation shows that the size distribution of heterogeneities that could be responsible for the observed activation times is remarkably similar to the detected blood vessel size distributions.

\subsubsection{Universality of activation time scaling}

In section 3.3.1, it was motivated that the blood vessel size scaling is a result of certain physiological constraints. In the range of sizes reliably detected by $\mu \mathrm{CT}$ measurements, these constraints led to a power law PDF for blood vessel radii. Via the described wave source recruitment mechanisms, this scaling behavior is transferred to a characteristic field-strength dependence of optically measured activation times. At this point, the question arises how universal this scaling behavior is. Does it extend to other species? And, even if this might not be surprising, how does the power-law behavior depend on the pulse shape of the applied electric-field? For the in-vitro investigations in canine hearts shown above, monophasic rectangular pulses were used throughout, because this waveform was thought to be most directly connected to the theory for wave emission from heterogeneities. However, in modern ICDs, biphasic pulses are used, because these have been shown to result in considerably higher success rates for the same energy [234]. Given that the adjustable wave source density observed in the previous sections is thought to be an important prerequisite for the efficacy of LEAP, the question of universality of the activation time scaling is also important with respect to the clinical applicability of this low-energy technique.

For this reason, activation maps were obtained in isolated ventricular preparations of pig hearts. The hearts were excised from euthanized pigs after in-vivo experiments investigat- 
a)

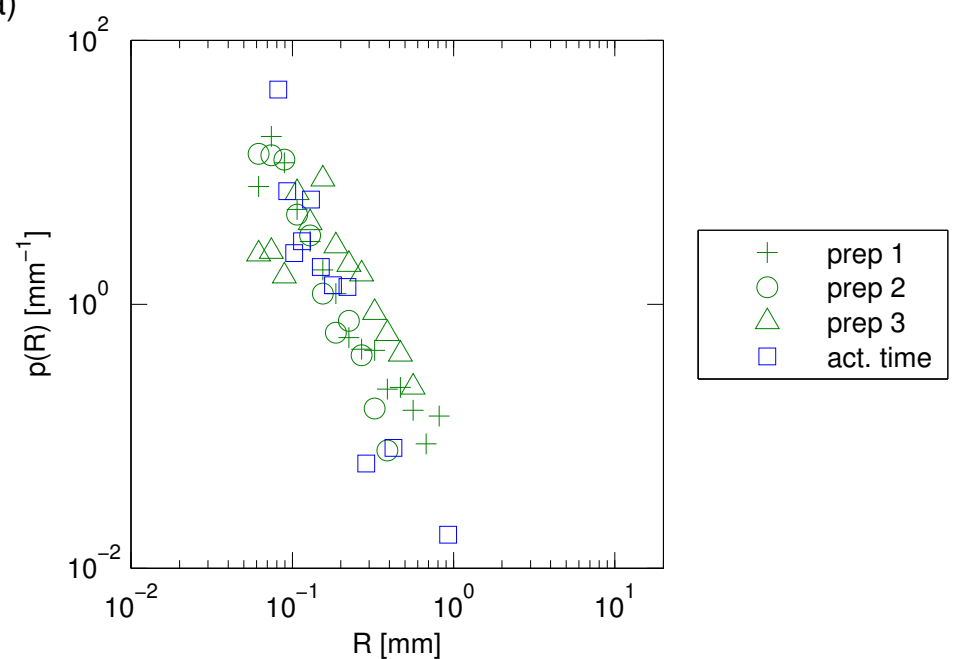

b)

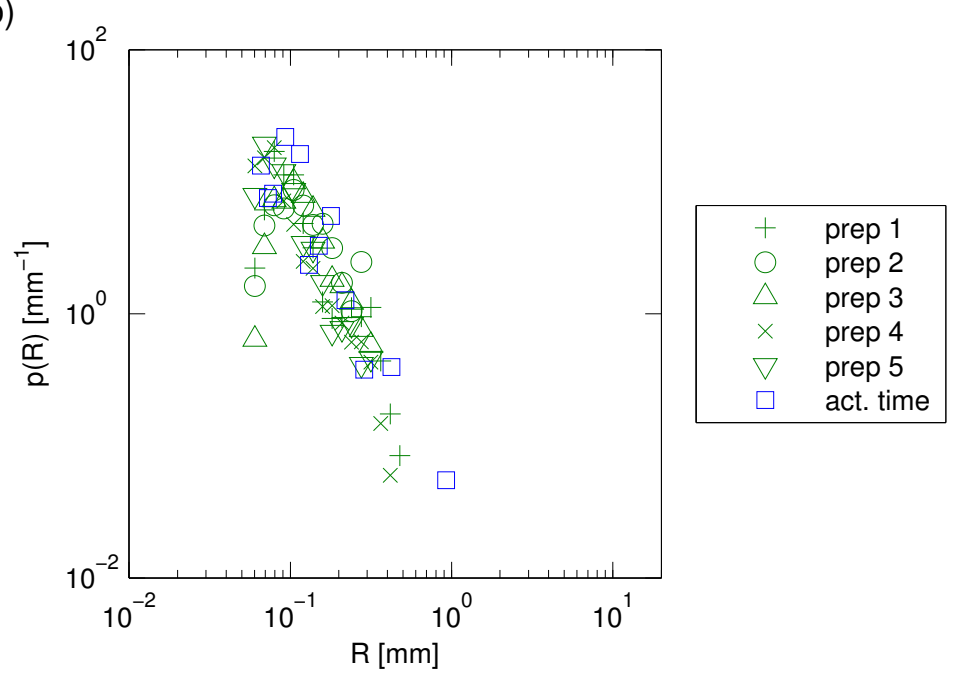

Figure 3.45: Size distribution estimation from canine activation times. a) Size distributions for all three ventricular preparations (green symbols) and size distribution calculated from averaged ventricular activation time data (blue squares). b) Size distributions for all five atrial preparations (green symbols) and size distribution calculated from averaged atrial activation time data (blue squares). 
ing the application of LEAP to ventricular fibrillation in healthy animals which are carried out in collaboration with the cardiology department of the University Hospital Göttingen. The optical mapping setup at Göttingen is functionally identical to that one used for the canine heart studies. For these particular experiments, the custom-made electrodes used to deliver the electric field-pulses in the dog experiments were physically transferred to Göttingen, to increase the comparability of the experiments by using the exact same field configuration. For these experiments, the averaging procedure for the activation times of different trials is more straight forward (no binning required), as all measurements were carried out using the same, automated voltage-stepping protocol controlled by an improved LabView program. The tissue response was recorded ten times for each voltage and activation maps were averaged. Additionally, to compute the activation time, the mask (cf. figure 2.13) was restricted to those locations in the recorded video which showed a fractional change in optical signal intensity of $1 \%$ or more. Two different wave forms were used: biphasic rectangular pulses and biphasic truncated exponential pulses (see figure $2.12 \mathrm{~b}$ ) and c), respectively). The latter resemble those applied by capacitor-discharge based modern defibrillators and by the experimental defibrillator device used in the corresponding in-vivo experiments. The pulse duration used throughout is $T_{\text {pulse }}=10 \mathrm{~ms}$, the exponential decay rate was set to $\tau_{\text {decr }}=4.95 \mathrm{~ms}$, which roughly corresponds to the time scale observed in the in-vivo experiments.

Figure 3.46a) shows the measured activation times for both wave forms, plotted against the peak voltage of the pulse (see figure B.3, page 181, for individual measurements). The log-log plot of figure $3.46 \mathrm{~b}$ ) indicates that the activation times accurately follow a power law over a wide range of values. The small error bars even at activation times around $20 \mathrm{~ms}$ indicate the superiority of the newly developed automated experimental protocol based on its increased reproducibility and better statistics due to a larger number of measurements. However, even here, large variability is observed below a critical field strength, which is accompanied by a clear deviation from the power law behavior. As before, this indicates that other wave source recruitment mechanisms might become important in this field strength range. The dashed lines represent power laws with almost the same exponent (see legend), which demonstrates that the pulse shape has negligible influence on the observed power laws, apart from an overall shift of the curve towards higher voltages. This shift results from the reduced effective pulse amplitude for the exponential case compared to the rectangular wave form, since the amplitude variations on a time scale comparable to the membrane time constant will be averaged out. This effect can be included by the same procedure as in section 3.2.13, converting the peak voltage to a mean voltage for each half of the biphasic pulse. The collapse of the two curves in figure 3.46;) indicates that, indeed, only the mean voltage (or, equivalently, the total injected current) determines the activation time, and therefore the wave source density. Hence, the time scale for the depolarization of the tissue seems to be on the same order as the duration of one polarity of the stimulus ( $5 \mathrm{~ms})$.

\subsubsection{Brief summary}

In section 3.3 , the wave source recruitment mechanisms underlying low-energy electricfield based control of cardiac tissue was investigated. The hypothesis outlined in sec- 
a)

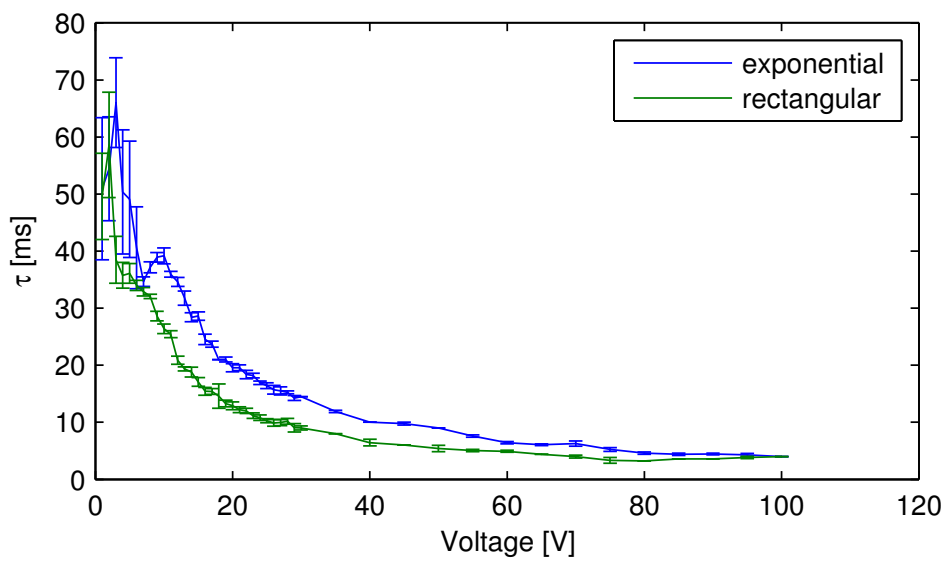

b)

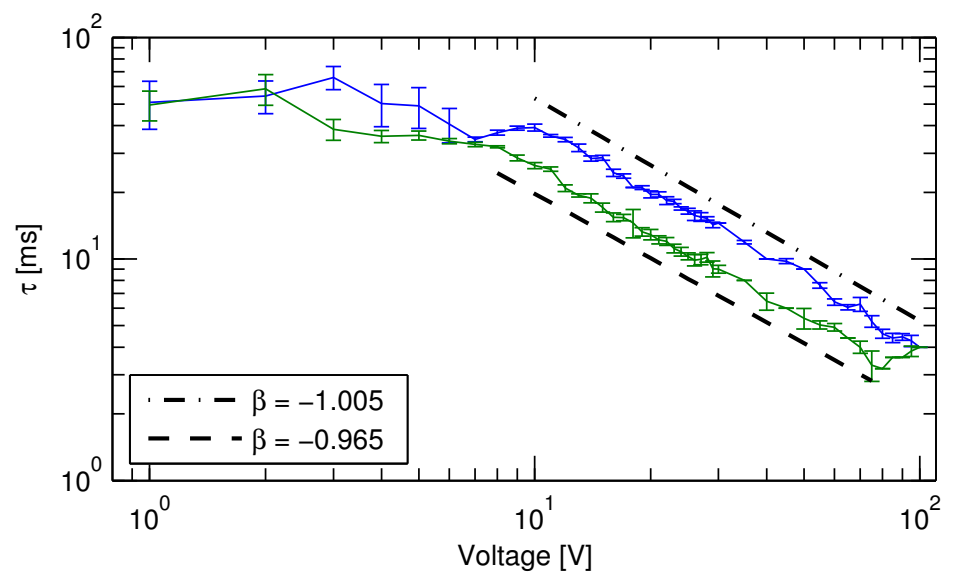

c)

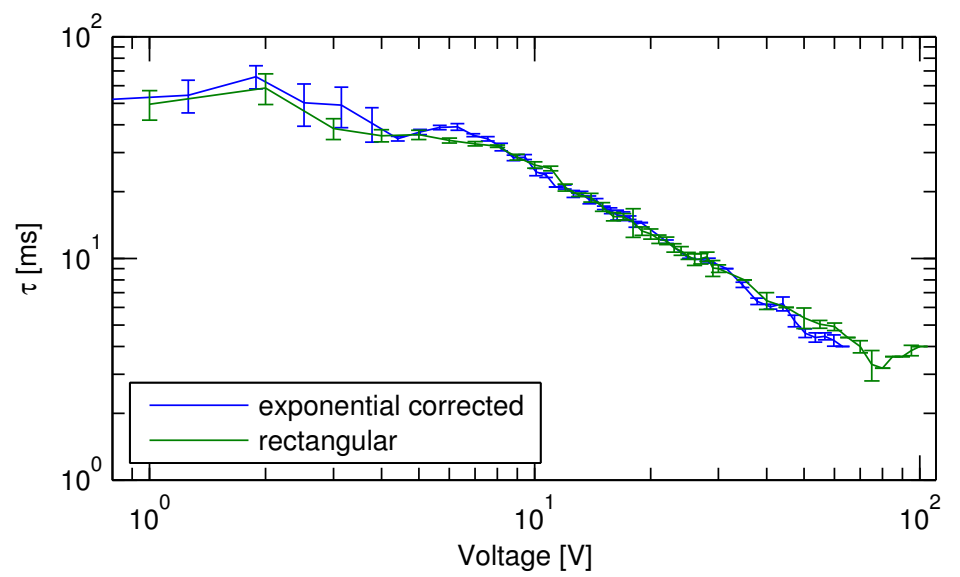

Figure 3.46: Activation time scaling in pig ventricular preparations. a) Average of multiple activation time measurements in two ventricular wedge preparations of excised pig hearts, using biphasic electric-field pulses with rectangular (green, $T_{\text {pulse }}=10 \mathrm{~ms}$ ) and truncated exponential (blue, $T_{\text {pulse }}=10 \mathrm{~ms}, \tau_{\text {decr }}=4.95 \mathrm{~ms}$ ) shape. b) The same data in a log-log plot. Dashed lines indicate power laws obtained by least-squares fitting (with offsets for better readability). c) Correction of the voltage scale for the truncated exponential pulse shape according to equation (3.53) yields almost identical activation times for both cases. See figure B.3 for plots of all individual activation time curves. 
tion 3.3.1 was that the dominant source of heterogeneity underlying wave emission in the field strength range relevant for LEAP is the tree of cardiac vessels. To test this hypothesis, a model based on the idea of homogeneously distributed wave sources was constructed in section 3.3.2, which links the size distribution of cardiac vessels to the dynamics following an electric-field stimulation by utilizing the theoretically predicted, radius-dependent threshold field strength necessary for successful recruitment. In sections 3.3 .3 and 3.3.4, structural and dynamical data for multiple preparations of canine ventricles and atria was presented, which were obtained from $\mu \mathrm{CT}$ measurements and optical mapping experiments, respectively. In section 3.3.5, it was shown that these experimental size distributions $p(R)$ and activation times $\tau(E)$ can be linked successfully using the above model in the form of equations 3.59 and $(3.62)$. Alternatively, the scaling exponents of the two kinds of data were related to each other using equation 3.65, eliminating the need for assumptions about the correct physiological values for $\lambda$ and the excitation threshold. Both observations provide significant experimental evidence that the cardiovasculature is the dominant kind of heterogeneity for the field strength range in which activation times were consistent across preparations. For lower field strengths, preparation-specific heterogeneities become relevant (cf. section 3.2 [218]), leading to a greater variability in the observed activation times. Finally, in section 3.3.6, activation time data obtained in optical mapping experiments on pig ventricular preparations was presented. Although no size distributions of the cardiovasculature were available yet to quantitatively check the relation between structure and dynamics for these preparations, the activation time data suggests that its power-law behavior is a universal feature across species and does not depend critically on the pulse shape used to stimulate the tissue in terms of the injected electric current. For a detailed summary and a discussion of the results, see section 4 . 


\section{Chapter 4}

\section{Conclusion}

The work presented in this thesis was centered around the complexity of the heart, which becomes manifest in both its structure and its dynamics. The function of the heart was examined from different perspectives: The subject of section 3.1 was the characterization of dynamical complexity in the heart viewed as a heterogeneous excitable medium, whereas in sections 3.2 and 3.3 the relevance of a specific kind of structural heterogeneity, namely tissue boundaries, for electric-field based control strategies was investigated. In this section, the results of all three studies shall be summarized (section 4.1) and discussed (section 4.2), pointing out their respective limitations and possible directions of future research. Finally, the findings will be put into context by considering their contributions to the long-term vision of a specific diagnosis of cardiac arrhythmias and subsequent modeldriven, tailor-made therapies.

\subsection{Summary}

In the beginning of section 3.1, a fundamental understanding of the structure of the Lyapunov spectrum for typical attractor types in excitable media was established. It was shown that Lyapunov stability analysis can successfully be applied to the Barkley model and the smooth Fenton-Karma model, the latter of which implements the most important characteristics which are specific to cardiac tissue. In both cases, well-defined leading Lyapunov spectra are obtained, which become independent of the discretization of the extended system for sufficient resolution, indicating low-dimensional attractors in the infinite-dimensional phase space of the original PDEs. Further investigations in the Barkley model showed that periodic and quasi-period orbits can be reliably identified from the structure of the Lyapunov spectrum and bifurcation points can be detected in the same way as in low-dimensional systems. In addition, the excitable nature and the spatial topology of the system leave the following marks in the Lyapunov spectrum: Firstly, as the degrees of freedom of the extended system do not only form an abstract phase space but can be identified with certain locations in physical space, the same is true for the Lyapunov vectors. In agreement with the excitable nature of the dynamics, Lyapunov vectors for the leading exponents are concentrated at wave fronts and backs, with characteristic oscillations for higher-index exponents, whose values for plane waves were explained by the action of diffusion with an analogy to systems of coupled oscillators. For spiral waves, these oscillations could be identified as the meandering modes. Secondly, 
the dimensionality of the neutrally stable subspace is tightly connected to the symmetries (and therefore to the topology) of the system and the wave pattern. In particular, the preservation of these symmetries for multiple rotating waves in the same system opens a new way of defining the entity of a spiral wave and its domain of influence, independent of any definition of a phase necessary to identify these object via phase singularities. In fact, it was found that, at least in the Barkley model, the upper Lyapunov spectrum of multiple spiral waves is identical to that of multiple systems containing a single spiral wave, which indicates a mutual decoupling of the parts of the medium controlled by different spirals. Furthermore, the convergence of the leading Lyapunov spectrum for increasing numerical resolution provides an independent measure for the faithfulness of the numerical approximation.

Utilizing the knowledge about these fundamental properties of the Lyapunov spectrum in homogeneous media, the effect of attracting heterogeneities in excitability was studied. The Lyapunov spectra obtained for spiral waves interacting with discrete heterogeneities of excitability reveal that there is an interplay of effects determined by the size and strength of heterogeneity. Opposing tendencies of stabilization (symmetry modes) and destabilization (meandering modes) are present for different perturbation modes, in the extreme case leading to a heterogeneity-induced meandering bifurcation, which is consistent with an experimental finding by FAST and PERTSOv, who observed drift along the border of an inhomogeneity [235]. Similar behavior was observed for randomly distributed and arbitrarily shaped heterogeneities, with additional destabilizing effects on lower Lyapunov spectrum below the meandering modes. Meandering was only observed for the scenario of an abrupt heterogeneity in excitability.

Finally, the influence of discrete heterogeneities in excitability was examined during a state of spatio-temporal chaos in the Bär-Eiswirth model with the help of the Lyapunov dimension, which can be regarded as a measure for dynamical complexity. It was found that, although reduced excitability leads to increased complexity of the chaotic activity in a homogeneous medium, this effect is absent for patches of low excitability up to a certain size, indicated by a constant Lyapunov dimension. As for stable spiral waves, the size of the heterogeneities was found to be a critical factor: For a resonant size of heterogeneous regions, the chaotic activity was even found to be stabilized, for strong heterogeneities eventually leading to a highly organized state of multiple pinned spiral waves.

The investigations in section 3.2 were focused on the influence of tissue geometry on the depolarization pattern induced by electric fields. Using analytical methods and an extension of the phase-field method (section 2.1.5), a low field strength, steady-state approximation to the cardiac mono-domain equations, originally developed by A. PUMIR and V. KRINSKY for circular obstacles to electrical conduction [127, was generalized towards boundaries of arbitrary shape. The results [218], supported by cell-culture experiments, reveal a strong correlation of the sensitivity of the tissue with anatomical features of the substrate, based on an interplay of different mechanisms governing the response of cardiac tissue to electric-field stimulation: curvature effects, interaction of depolarized and hyperpolarized regions, and the scale of anatomical features with respect to the electrotonic space constant $\lambda$.

Relative to the electric-field induced depolarization next to a straight boundary, elec- 
trotonic effects lead to an enhancement of the maximum tissue depolarization towards negative curvatures and a decline towards positive curvatures. This is qualitatively in line with a series of cell culture experiments revealing an increased sensitivity of negativecurvature boundaries. Theoretically, the relevant quantity determining the strength of this curvature effect in the vicinity of curvature zero was determined to be the normalized mean curvature $\kappa^{*}$ (in both two and three dimensions). In the natural, normalized units of the problem, this difference to the depolarization of a straight boundary was shown to be just $-\kappa^{*}$. For larger deviations from a straight boundary, the global shape of the tissue becomes important: while for parabolic boundaries or protrusions, the maximum depolarization caused by an electric field is monotonic in the curvature, there is a maximum effect strength for a specific value of (negative) curvature for circular boundaries, caused by the interaction of the "pure" curvature effect and the spatial vicinity of depolarized and hyperpolarized boundaries. For small tissue domains of arbitrary shape (on a length scale much shorter than $\lambda$ ), this interaction was shown to result in a maximum depolarization proportional to the length of the domain in the direction of the electric field. An analytical argument was presented which makes it possible to transfer the results for oneor two-dimensional tissue domains to higher dimensions.

In numerical simulations based on the Fenton-Karma model, two restrictions of the theory (infinitesimal field strengths and infinite pulse duration) were determined not to be essential for its applicability to realistic scenarios of electric-field stimulation of cardiac tissue with a complex geometry. Qualitatively, the higher sensitivity of negative-curvature boundaries, and therefore its response to lower electric fields, could be confirmed in those simulations.

In a systematic study for circular boundaries, it was shown that there are quantitative differences in the field strength necessary for wave induction, which, for an experimentally relevant pulse duration, depend on the pulse shape. However, the existence of an optimal radius for circular tissue regions was found, though for slightly different values of the curvature, to be independent of the pulse shape. The differences in critical field strength between different stimulation wave forms could be explained with a shape-dependent time scale for the electric-field induced depolarization next to a tissue boundary. This time scale was found to be in agreement with the time scale on which the membrane potential approaches the steady-state solution in the low field strength approximation. Broadly speaking, high- $\left|\kappa^{*}\right|$ boundaries were found to depolarize on smaller time scales, leading to only small differences in the critical field strength between biphasic and monophasic pulses. At the same time, the short time scales result in an advantage of truncated exponential wave forms in terms of the average field strength necessary for wave induction, because the effect of the initial high field strength implied by the wave form is more pronounced. In contrast, longer time scales for small- $\left|\kappa^{*}\right|$ boundaries lead to a larger difference between biphasic and monophasic pulses, while simultaneously canceling out the advantageous effect of the exponential wave form.

In the experimental investigation presented in section 3.3 , the recruitment mechanism for intramural wave sources underlying LEAP was studied. The hypothesis was that the cardiovascular tree provides the heterogeneities in conductance giving rise to multiple pacing sites for LEAP, which act as control sites for spatio-temporal chaos during fibril- 
lation. To test this hypothesis, isolated cardiac tissue preparations of canine atria and ventricles were artificially perfused and observed in-vitro using the technique of optical voltage mapping.

The theoretical wave source density was calculated from size distributions of the coronary vessels, which were obtained from $\mu \mathrm{CT}$ scans of the tissue preparations perfused with contrast agent. Based on the size statistics, in accordance with the results of section 3.2 , the asymptotic theory for circular heterogeneities developed by A. PUMIR and V. KRINSKY [127] was utilized to theoretically determine the fraction of (cylindrical) blood vessels activated at a certain electric field strength. A simplistic model was constructed connecting a specific wave source density in the bulk of the cardiac muscle to an observed activation time. In the in-vitro experiments, this proxy quantity was directly measured following the stimulation of quiescent tissue with short electric-field pulses.

Both the size distributions and the activation times as a function of the field strength were found to follow power laws, whose exponents could be connected analytically to each other, separately for atria and ventricles, with good quantitative agreement. Even better consistency could be reached by using the above model to predict activation times directly from the measured size distributions. The inverse relationship was used to construct hypothetical size distributions of circular heterogeneities which could be responsible for the observed activation times. Again, good quantitative agreement with the real size distributions of the cardiovasculature was found. These results [201] provide a strong empirical basis for the relevance of the cardiovascular tree as wave nucleation sites during LEAP in the considered field strength range.

Using the same experimental technique, but an improved and computer-controlled protocol, activation times were also measured in perfused ventricular preparations of pig hearts. Although size distributions of the coronary vessels were not available yet, the optical mapping experiments indicate that the power law behavior of the activation times is a universal feature across species and different pulse shapes, which suggests that LEAP efficacy should not depend fundamentally upon species or the technical implementation details of the defibrillator. Quantitatively, it was found that for pulse durations of $10 \mathrm{~ms}$, the total injected current alone (or, equivalently, the mean electric field strength) determines the density of recruited wave sources, independent of the pulse shape.

\subsection{Discussion and Outlook}

The aim of section 3.1 was to establish Lyapunov stability analysis as a new tool for the characterization of the dynamics in models of cardiac tissue or, more generally, excitable media. As outlined in the introduction, the study of the stability of activation patterns is an attempt to conceptually understand how undesired spatio-temporal activation patterns arise and to quantify the complexity of such dynamics. The work presented in the first subsections of section 3.1 can be viewed as a methodological proof of principle in this context. It shows that the acquisition of Lyapunov spectra in numerical models of excitable media with a vast number of degrees of freedom is feasible and leads to meaningful results despite the infinite-dimensional phase space of the underlying PDE. Its main advantage over the linear stability analysis of fixed points is that it can be applied to arbitrary trajectories and does not require any considerable algorithmic effort. Although 
the computational demand increases with the number of perturbation modes integrated along with the system dynamics, by construction, reaction-diffusion systems are easily linearized, as the Jacobian of the (relatively low-dimensional) local dynamics can usually be calculated analytically, and diffusion acts in the same way as in the original system.

To systematically gain an understanding of the meaning of Lyapunov spectra for these systems, relatively simple scenarios in the Barkley and (smooth) Fenton-Karma model were considered in the beginning of section 3.1 , in particular to make the transition from existing results of the linear stability analysis of fixed points [106, 107, 108, and response functions [109, 81]. Future studies could be extended to other models of cardiac tissue, including spiral waves with non-circular cores and instabilities leading to breakup [104]. Moreover, deviations from the scenario of multiple independent spiral waves presented in section 3.1.3 could reveal valuable information on the mechanisms governing the interaction between spiral waves in more realistic models of cardiac tissue. These could turn out to be important for strategies aiming at the termination of individual spiral waves, depending on whether they can be viewed as independent waves or have to be considered "entangled" in some way.

From a methodological point of view, one important aspect of the method is that destabilizing effects can already be detected before the modes actually become unstable, something that would not be possible by simply observing the dynamics: For example, in figure 3.12 (page 99), for stable spiral waves, the rate with which the meandering modes are destabilized indicates, whether there is a danger of arriving at a bifurcation (in this case, the nature of the bifurcation could even be identified from the spatial structure of the corresponding Lyapunov vectors). Similarly, during spatio-temporal chaos, the subtle stabilizing effect of heterogeneities in excitability was already detectable via the Lyapunov dimension for small heterogeneities, but visible effects like spiral wave pinning or reduced number fluctuations of phase singularities were only observed for specific sizes of heterogeneities (cf. figure 3.19, page 110). In the future, this predictive power of Lyapunov stability analysis could help to identify the critical directions in the usually large parameter space of detailed, physiological, cardiac models which make the dynamics more vulnerable to instabilities. Similarly, it could facilitate the search for possible countermeasures suppressing a developing instability or reducing the complexity of a turbulent state.

The results on the influence of heterogeneities presented in sections 3.1.5, 3.1.6 and 3.1.7 point to a whole new class of interesting but difficult questions regarding the proarrhythmic or antiarrhythmic effect of local changes in the properties of cardiac tissue. Similar to the effect of increased $\epsilon$ in the Bär-Eiswirth model (in a homogeneous medium), it is a generally accepted fact that reduced excitability in cardiac tissue is proarrhythmic [236]. The results presented here suggest that it is, in general, not possible to predict the effect of a local parameter change from a known behavior in a homogeneous medium. Thus, if excitability is reduced in a spatially heterogeneous manner in the heart, the effects might differ greatly from those observed for global changes of physiological conditions or expected on the basis of mean tissue properties. Furthermore, the results show that the effects could depend in a non-trivial manner on the length scale and the strength of the heterogeneity. As meandering for a single spiral wave was only observed for the step-wise heterogeneity considered in section 3.1 .5 but not for the random, smooth heterogeneities imposed in section 3.1.6, the steepness in the change of properties towards the heteroge- 
neous region apparently is another determining factor. Heterogeneity-induced complexity changes during spiral wave or chaotic activity could be of particular relevance for the termination of tachycardia or fibrillation in diseased hearts, when the natural heterogeneity is increased, either facilitating or impeding low-energy termination efforts. An investigation of such effects should be carried out by simultaneously considering the results of section 3.3 and the effect the additional heterogeneity might have on wave source recruitment during electric-field stimulation.

One should bear in mind that spiral waves in the ventricles are organized around threedimensional filaments that evolve in a medium of irregular shape, which adds considerable complexity to the problem. However, following the mathematical line of thoughts outlined in section 2.2.6, it should in principle be possible to apply Lyapunov stability analysis to numerical simulations of cardiac activity on realistic geometries. In this way, the complexity of fibrillation and its influence on controllability by low-energy methods could be tested in a realistic scenario. Another possible application of Lyapunov analysis for realistic simulations could be based on the analogy of plane waves to the synchronization of coupled oscillators: The creation of a reentry pattern underlying tachycardia requires the break of the plane wave. This process could be viewed as a destabilization of synchronization between pathways in the heart which, under normal conditions, are activated in parallel. In view of the recent popularity of covariant Lyapunov vectors, another possible application of Lyapunov stability analysis is during spatio-temporal chaos. While in section 3.1. most of the Lyapunov vectors (except for the meandering modes) were located in the degenerated subspace of the largest Lyapunov exponent, which is automatically covariant, general covariant Lyapunov vectors have been shown to allow for an independent definition of an effective physical dimension in other extended systems exhibiting spatio-temporal chaos [117] and their spatial structure was used to identify relevant length scales and locations in the system [115]. In view of the latter, it is therefore possible that a more detailed inspection of the Lyapunov spectrum and the spatial structure of covariant Lyapunov vectors in spatio-temporally chaotic excitable media (as considered in section 3.1.7) could reveal the mechanism underlying the strong size dependence of the effect of heterogeneities. Additionally, they could contain information about the optimal number and location of control sites necessary to suppress chaos.

One way to noninvasively create such control sites in the tissue is via virtual electrodes in response to electric fields. The creation of these secondary sources at tissue boundaries was the subject of section 3.2. A particular sensitivity to electric fields was predicted for negative-curvature boundaries, which correspond to convex tissue regions and therefore outer boundaries of the tissue. The most obvious consequence of the theoretical predictions is that, assuming the electric field is appropriately oriented, the activations that are observed for the lowest field strength, should come from certain locations on the endocardial surface, which has a particularly complex anatomical structure (see introduction). Depending on the concrete spatial arrangement and their attachment to the endocardial surface, trabeculae and papillary muscles can be caricatured as protrusions from the endocardial surface or as cylindrical tissue domains. The non-monotonic shape of the green curve in figure 3.27 (page 127) singles out a particular size from these anatomical features, which is optimal in terms of electric-field sensitivity. The importance of structures that 
are linked to the bulk of cardiac tissue in distant places for the activation dynamics has already been shown for the Purkinje system, which was reported to have an increased excitability compared to the bulk of tissue due to it being a one-dimensional geometric structure [237. The results therefore suggest that there are even more structures in the heart that, only due to their geometry, can be excited very easily. Optical mapping studies of the complex endocardial surface could verify this spatially heterogeneous response to electric fields.

With respect to low-energy control of cardiac activity, the results indicate that further significant energy reduction may be achieved by control strategies that aim at inducing wave emission from the above-mentioned structures. However, being located on the endocardium, they provide direct access to a very specific tissue region near the inner surface of the heart, only. Simulations including ongoing activity in the tissue and experiments will have to determine whether control sites on the endocardium could provide an effective way of controlling simpler arrhythmias than fibrillation, e.g., polymorphic or fast ventricular tachycardias, thereby filling the gap in between ATP and LEAP. Additional effects going beyond the scope of the framework presented here can be expected, as dynamical properties such as the action potential duration can be influenced by the presence of boundaries as well [238.

While most of the results presented in section 3.2 are independent of the actual membrane kinetics, because the theory is based on a linearization around the resting state, the simulations for finite pulse duration and different pulse shapes in section 3.2 .13 indeed do depend on the specific model. The Fenton-Karma model was chosen because it has been shown to produce results comparable to more detailed, ionic, models. However, quantitatively, this comparability has only been shown for reentry patterns and restitution properties [84, i.e. tissue properties which are defined for propagating action potentials. It is therefore unclear, whether the subthreshold dynamics, which is important for the critical electric field strength determined in section 3.2.13, is realistic, too. Indeed, one degeneracy of the model is that the membrane dynamics is completely passive below the excitation threshold $u_{c}$, except for the slow inward current, which activates slowly towards $u_{c}^{\text {si }}$. This absolute passivity leads to the seemingly plausible and large underestimation by the asymptotic theory of the electric field necessary to activate circular boundaries of all curvatures in figure 3.35 (page 139). If the sodium channels responsible for the fast inward current were modeled in a stochastic manner, the force driving the membrane potential back to its resting value would decrease towards the excitation threshold. This could lead to a narrowing of the gap between the critical field strength determined from the numerical simulation and that predicted by the analytical theory.

Most of the results presented in section 3.2 are based on theory and numerical simulations. However, it should be emphasized that there is growing experimental support for the theoretical framework in several aspects: The original theory, developed by A. PUMIR and V. KRINSKY to describe the effect of circular obstacles in the tissue [127, was not only successfully employed in section 3.3 of this thesis to make experimentally relevant predictions for low-energy stimulation of cardiac tissue, but was also tested in a more direct way using cardiac cell cultures: In her $\mathrm{PhD}$ thesis, C. RICHTER measured critical electric field strengths for the activation of artificial circular obstacles as wave emitting sites in cardiomyocyte cell cultures of neonatal rats [239], thereby confirming the quantitative 
predictions of the theory (combined with results from reference [240]). The results of the generalized theory presented in this thesis contain two more predictions which are in line with experimental results: Firstly, the high sensitivity of negative-curvature boundaries was qualitatively confirmed by the cell culture experiments presented in section 3.2.5. Further cell culture studies with carefully-prepared artificial boundaries of known curvature could take this verification to a quantitative level or indicate directions of improvement. Secondly, the reduced time scale for depolarization next to high-curvature circular boundaries found in section 3.2 .13 is consistent with a recent study by A. KONDRATYEV et al., who found a considerably shorter time scale for the rise of the membrane potential in cell cultures of neonatal rat myocytes, if depolarized and hyperpolarized straight boundaries are in close proximity [221]. Thus, a qualitatively similar mechanism seems to be in effect for curved boundaries.

When drawing conclusions from the results in section 3.2 about the absolute sensitivity of certain types of boundaries to electric fields, one should bear in mind that several approximations were necessary to derive the central equation (3.16) (page 116) on which all theoretical predictions are based. One assumption is that the outer potential at the boundary is a simple gradient leading to a constant electric field vector. In section 2.1.3. it was argued that one way to justify such a fixed potential is by assuming very large extracellular and/or outer conductivities. This could be a good approximation for cell cultures, where a monolayer of cardiomyocytes is usually covered by a well-conducting layer of culture medium. In real tissue, however, the conductivities in the extracellular space are usually on the same order of magnitude as the intracellular conductivities [32], which renders the assumption of a decoupled extracellular potential unrealistic. A possible extension of the theory is therefore to account for the "feedback" of the dynamics on the extracellular potential. If this should not be possible analytically, only full bi-domain simulations (including a third, passive, outer domain) can determine the effect of this approximation. Likewise, the influence of tissue anisotropy (see sections 1.3 and 2.1.4) on the results presented in section 3.2 could be studied in such simulations: While the results are not expected to change qualitatively for the case of equal anisotropy ratio (cf. section 2.1.4), it is known from an extension of the original theory for circular obstacles that an unequal anisotropy ratio can lead to the appearance of additional zones of depolarization and hyperpolarization further away from the boundary [219, 128]. Once the approximation used here has been validated in bi-domain simulations, the extended phasefield method developed in section 2.1.5 for arbitrary boundary conditions could prove to be a valuable tool for the efficient numerical simulation of cardiac tissue in the future, by eliminating the need for full bi-domain simulations when the effect of an electric stimulation is known to be implementable via modified boundary conditions.

In addition to the above-mentioned systematic deficiency of the theory, it should also be emphasized that the electric field assumed in equation (3.16) implicitly contains the conductivity ratio $\frac{\sigma_{o}}{\sigma_{e}}$ of the outer conductivity and the extracellular conductivity (see, e.g., equation (3.13). Therefore, the electrical interface configuration can also influence the absolute effect strength of the electric field. If the outer domain is identical to the extracellular domain (i.e., when the cellular substrate is continuous and only the intracellular space ends at the boundary), the ratio is equal to one and the theory applies unchanged. However, if the extracellular space is connected to a well-conducting open space (e.g., to 
a bath) at the boundary, this will enhance the effect of the electric field. Again, this simplified rule is only valid under the assumption of a fixed electric field strength in the outer medium. In general, a well-conducting outer medium will have a smaller potential gradient if the total voltage across the whole setup (tissue and outer medium) is determined by two electrodes at fixed potentials, diminishing or even overcompensating the above effect. Even for internal boundaries, interface conditions might be altered for anatomical or physiological reasons, such as endothelial cells covering blood vessels or a different tissue composition in papillary muscles and trabeculae (see sections 2.1.3 and 2.2.3 in reference [32]).

Despite these complications, it was shown in section 3.3 that the theory for virtual electrodes near tissue boundaries can be used to predict the global response of cardiac tissue to electric fields from anatomical data. The knowledge that blood vessels are the relevant heterogeneities responsible for intramural activation of the tissue during LEAP can aid the modeling and optimization of this low-energy control strategy. Furthermore, the large number of wave sources which can be adjusted by choosing an appropriate electric field strength provides a plausible explanation for the efficacy of LEAP, by enabling the creation of a control site density which is just sufficient to terminate a given arrhythmia. The results of section 3.2 could help to include shape-dependent boundary activation into the prediction and thereby provide a deterministic explanation for some of the large variability of activation times in the very low field strength range seen in figures $3.41,3.43$, 3.44 and 3.46. However, to build a theoretical model for the efficacy of LEAP, which predicts the outcome of a termination attempt on the basis of the chosen pacing parameters, it is necessary to gain a more detailed understanding of how the activity initiated at blood vessels and other heterogeneities interacts with the spatio-temporally chaotic activity of the muscle during fibrillation.

One of the mechanisms which could play an important role in this respect is that of unpinning: As seen in section 3.1, the pinning force of a heterogeneity increases with decreasing excitability. Circular obstacles can be viewed as the extreme case of a completely inexcitable region, whose tendency to attract spiral or scroll waves was recently quantified by ZEMlin and PerTsov [79]. These anchored spiral waves present a particular challenge for termination by high-frequency pacing (ATP) from a location far away from the anchoring site [241], as drift cannot be as easily induced as for freely rotating spirals [41]. It has been shown in several experimental and theoretical studies that inducing activity directly at the pinning location via electric-field stimulation can lead to unpinning of the wave [127, 128, 129, 130] and that the capabilities of this control strategy indeed exceed those of ATP [131, 132]. Along with the results presented in section 3.3, reference [201] makes out a good case for the importance of this mechanism during LEAP: a number of successful LEAP terminations of fibrillation using frequencies below the dominant frequency of the arrhythmia are a strong indication that the wave sources are located close the cores of the vortices underlying fibrillation (pacing from a distance should not be able to reach the spiral core in this case [41]). Hence, the cylindrical obstacles to electrical conduction represented by the cardiovasculature could be particularly effective in controlling fibrillation, because they are, at the same time, the heterogeneities to which scroll waves in the heart can pin. For geometrical reasons, pinning seems much less likely for other intrinsic 
heterogeneities such as intercellular clefts between the sheets of cardiac tissue. Based on this assumption, in a study with A. BEHREND, a model for the application of LEAP to a pinned spiral wave was constructed as a first step towards assessing the influence of the pacing frequency on the efficacy of LEAP [242]. However, one should not forget that wave emission is not the only mechanism by which heterogeneities can respond to electric field pulses. It has been proposed that effects such as the prolongation of APD or the repolarization due to virtual electrodes might also play a significant role in the defibrillating effect of an electric shock [100]. These investigations have to be reevaluated at the field strengths relevant for LEAP.

Due to the considerable energy reduction of LEAP compared to standard defibrillation reported in reference [201], it is a promising candidate for a painless defibrillation technique in future ICDs. However, since these devices are implanted in patients with diseased hearts who are at risk of developing serious arrhythmias, the investigation of the mechanisms underlying LEAP success have to be extended to altered physiological conditions, such as post-myocardial-infarction models with scar tissue or fibrosis. In the context of virtual electrodes, these additional heterogeneities could alter the activation patterns due to electric-field stimulation and, depending on their number and sizes, disturb the scaling behavior observed in healthy tissue in this thesis. On the other hand, they also influence the development and evolution of arrhythmias in the tissue, as indicated by the results presented in section 3.1, for example by attracting spiral waves or reducing the complexity of fibrillatory activity. Thus, there is no fundamental reason why the same favorable line of thoughts as indicated above for the pinning and unpinning of vortices should not apply to these heterogeneities, but, of course, this has to be verified experimentally. With respect to the model for wave source recruitment presented in section 3.3 , estimating the tissue response for other kinds of heterogeneities or in other species (cf. figure 3.46) from structural data will be a good test for the general theoretical framework.

While the quantitative agreement of predictions and measurements in section 3.3 seems convincing, one should bear in mind the systematic errors introduced by the simplistic model to link size distributions and activation times, most prominently the reinterpretation of size distributions as homogeneously distributed obstacles, the ignoring of blood vessel orientation and overlapping activated regions, and the utilization of the unmodified asymptotic theory for virtual electrode formation. However, even if some of the errors are considerable, they might not necessarily lead to an immediate invalidation of the results: For example, a misestimation of the absolute field strength necessary to excite blood vessels of the measured sizes (due to wrong physiological parameters used in the model, specific interface conditions at blood vessel boundaries, etc) would leave the conclusion untouched, if one assumes that the scaling behavior of blood vessel radii extends to smaller sizes as well. Size statistics of smaller blood vessels and high-resolution, three-dimensional numerical simulations incorporating blood vessels of the full range of sizes could help to unravel the effect of the different theoretical approximations. In addition, experiments employing novel imaging techniques could provide a way to directly observe wave sources and localize them in the three-dimensional myocardium [243].

From the data of pig ventricular activation times shown in figure 3.46 , it is evident that the automation of the experimental protocol alone can lead to considerable improvements in the reliability of activation time measurements. In retrospect, the very robust power- 
law behavior observed there can also serve as a justification for assuming a power-law behavior in the relatively sparse data of the canine hearts in the first place (compared with the pig hearts). Blood vessel size statistics for the pig hearts, as soon as they become available, will thus allow for more precise and definite statements about the compatibility of size distributions and observed activation times than was possible in the canine studies. Other possible technical improvements could be inspired by recent publications on a more reliable mathematical detection of power laws in experimental data [244, 245] and on a more accurate detection of surface activation times using optical mapping [246].

One aspect that deserves more attention in future studies is that of time scales: While the numerical results obtained in section 3.2 .13 show that the time scale for depolarization decreases substantially towards smaller heterogeneities, the independence of the activation time from the pulse shape indicated by figure $3.46 \mathrm{c}$ ) conveys a different impression: The fact that only the mean field strength determines the wave source density is a strong hint at a time scale on the same order of magnitude as the pulse duration across the whole range of voltages. It should be noted that the shape-dependent time scales in section 3.2 .13 were not only inferred from the difference in the critical field strength between different pulse shapes, but could also be extracted from the low field strength, steady-state approximation. Therefore, at least for the Fenton-Karma model, the time scales seem to remain correct when going from infinitesimal depolarizations to a scenario where the membrane potential crosses the excitation threshold. From this perspective, the experimental results of section 3.3.6 suggest that after the initial rise of the membrane potential (i.e. after leaving the linear approximation), when the membrane potential approaches the excitation threshold, additional ionic mechanisms not included in the Fenton-Karma model might have a dominating influence on the time scale. This deficiency could be related to the ionic passivity of the model below the excitation threshold mentioned above.

\subsection{Concluding remarks}

In this thesis, the dynamical and structural complexity of the heart was investigated from different theoretical and experimental perspectives. The results presented in the three subsections of section 3 have in common that they are based on reduced modeling approaches tailored to the respective problem: In section 3.1. homogeneous and heterogeneous excitable media on two-dimensional, rectangular geometries were considered to study the stability and assess the complexity of generic activation patterns corresponding to healthy or pathological cardiac dynamics. Section 3.2 was based on a mono-domain description of cardiac tissue combined with a boundary condition which incorporates the approximate bi-domain effect of a fixed electric field at the boundary. Tissue domains of idealized geometries were assumed to study the effect of boundary shape during electric-field stimulation. Finally, in section 3.3 , the cardiac muscle was modeled as continuum containing a homogeneous distribution of differently-sized heterogeneities which may emit waves of constant radial velocity upon recruitment by LEAP. The fact that such conceptually and methodologically different descriptions are used to characterize the same system already shows that none of the modeling approaches can possibly be regarded as complete, not to mention the aspects of the cardiac muscle which have been completely disregarded in this thesis: the molecular basis of the trans-membrane currents underlying cardiac electrophys- 
iology; the fast fibers of the electrical conduction system of the heart, most importantly for the ventricles: Purkinje fibers; the microscopic and macroscopic implications of contraction; the fluid dynamics of blood flow; and many more. However, reducing the model to the necessary ingredients for each problem allows for an easier determination of the mechanisms and prerequisites necessary to produce an observed effect. The hope is that the knowledge gained about these mechanisms in simple models within this thesis will aid their future identification in more realistic settings.

As outlined in the introduction, another benefit of a top-down approach to a complex system is that generic descriptions are very likely to be applicable to a broad class of systems. For example, the results presented in section 3.1 hold for excitable media in general and thus are valid for (and could also be tested in) other manifestations of this type of system, such as the chemical Belousov-Zhabotinsky reaction. Likewise, the effect of electric-field stimulation near boundaries studied in section 3.2 could also be relevant for other biological bi-domains, as the described curvature effects are solely based on the different extracellular and intracellular boundary conditions and the electrically excitable nature of the system. Both facts apply to any substrate composed of cells which are capable of action potentials. Possible applications include nerve fibers and neuronal tissue, which may have a complex shape and are stimulated with electric fields, e.g., during transcranial stimulation of the brain [247]. Although the model for wave source recruitment developed in section 3.3 is relatively specific, even here, generalizations are conceivable: Analogously to the results shown in figure 3.45, deducing the parameter distribution of wave sources from the activation times for other kinds of heterogneities should be possible, as long as a relation similar to equation $(3.60)$ is known that determines the minimum field strength (depending on the aforementioned parameter) necessary to recruit the heterogeneity as a wave source.

Returning to the concrete results of this thesis, the immediate purpose of the investigations was to refine the picture we have today of cardiac dynamics and the role of structural heterogeneity. Due to its complexity, the heart is a fascinating system to study for a physicist. Naturally, a deeper understanding of this vital organ is not purely academic but is particularly useful if it can aid the optimization or development of new therapeutic options. Each of the results presented in section 3 has the potential to contribute to a different aspect of future anti-arrhythmic therapies: Ultimately, the quantitative stability analysis investigated in section 3.1 could improve the risk assessment for the onset of arrhythmias and suggest a specific pharmaceutical intervention for known medical conditions based on indicators which have been determined using numerical models of cardiac tissue. For acute arrhythmias, similar indicators could be used to define and quantify the complexity of undesired activity. Also, Lyapunov spectra might be obtained directly from experimental data [248], although the high dimensionality of the system might impede such efforts. Once the dynamics of an acute arrhythmia has been characterized, suitable parameters for a tailor-made electrical intervention have to be chosen, which terminate the arrhythmia effectively but as gently as possible. These parameters include field strengths and time points of electric-field pulses. While on-demand simulations of an electrical shock to predict its effect and determine its optimal parameters will not be possible within the 
foreseeable future in an automated device such as an ICD due to high computational demands, only limited information and computational power are needed to determine the locations of induced wave sources for arbitrary field strengths from preprocessed anatomical data using the findings of sections 3.2 and 3.3 . The results presented in those sections, combined with the findings in reference [201] on the efficacy of LEAP, suggest that the field strengths leading to wave induction at the considered structural heterogeneities are exactly those suitable for the low-energy termination of arrhythmias. Probably, the information about the complexity of the dynamics and the spatial arrangement of wave sources is not sufficient to predict the outcome of the anti-arrhythmic intervention, but similar reduced models have to be obtained for the effect of the induced waves on the arrythmia in order to enable an accurate real-time assessment of the consequences. As an example in this direction, the success rate of low-energy pacing for different pacing frequencies can be determined with the help of a concept, again, from nonlinear dynamics, namely iterative maps, as demonstrated in reference [242. In this way, the results obtained within this thesis on the interaction of structural and dynamical complexity in the heart can open the way towards the vision of a patient-specific, model-based, low-energy treatment of cardiac arrhythmias. 


\section{Appendix A}

\section{Modeling details}

The parameters used for the Barkley (B), Bär-Eiswirth (BE) and Fenton-Karma (FK) model can be found in tables A.1 and A.2.

\begin{tabular}{c|c|c|c|c|c}
\hline \hline parameter set & B1 & B2 & B3 & B4 & BE1 \\
\hline$\epsilon$ & 0.02 & 0.02 & 0.02 & 0.02 & $*$ \\
$a$ & 0.9 & 0.9 & $*$ & 0.95 & 0.84 \\
$b$ & 0.08 & 0.06 & 0.05 & $*$ & $\begin{array}{c}0.07 \\
\checkmark\end{array}$ \\
Bär-Eiswirth modification & & & & & \begin{tabular}{c}
0 \\
\hline
\end{tabular}
\end{tabular}

Table A.1: Parameter sets for the Barkley (B) and Bär-Eiswirth (BE) model.

\begin{tabular}{c|c|c|c|c}
\hline \hline parameter set & FK1 & FK2 & FK3 & FK4 \\
\hline$k$ & 10 & 10 & 10 & 10.4222 \\
$k_{2}$ & - & 500 & 500 & - \\
$\bar{g}_{\mathrm{fi}}$ & 8.7 & $1 / 0.4050$ & 2.35 & 5.6093115 \\
$\tau_{0}$ & 12.5 & 8.3 & 8.3 & 8.36 \\
$\tau_{r}$ & 25 & 60 & 60 & 62.41 \\
$\tau_{\mathrm{si}}$ & 22.22 & 45 & 45 & 63.4124 \\
$\tau_{v 1}^{-}$ & 333 & 1000 & 100 & 40.4284 \\
$\tau_{v 2}^{-}$ & 40 & 3 & 3 & 36.5891 \\
$\tau_{v}^{+}$ & 10 & 3.33 & 3.33 & 10.8037 \\
$\tau_{w}^{-}$ & 65 & 9 & 9 & 132.373 \\
$\tau_{w}^{+}$ & 1000 & 97 & 97 & 663.715 \\
$u_{c}$ & 0.13 & 0.13 & 0.13 & 0.143713 \\
$u_{c}^{\text {si }}$ & 0.85 & 0.98 & 0.98 & 0.443994 \\
$u_{v}$ & 0.025 & 0.055 & 0.055 & 0.0420205 \\
\hline
\end{tabular}

Table A.2: Parameter sets for the Fenton-Karma (FK) model. If $k_{2}$ is specified, the continuous modification of the Fenton-Karma model introduced in section 2.1.6 is used, otherwise parameters refer to the original model as published in reference [84]. Parameter set FK1 is identical to GP parameters in reference [249]. Parameter set FK4 is the result of a fitting procedure of the model to restitution curves measured in rabbits by J. SchröDeR-Schetelig [224]. $\tau_{d}=C_{m} / \bar{g}_{\mathrm{fi}}$ with $C_{m}=1 \mu \mathrm{F} / \mathrm{cm}^{2}$ for all parameter sets.

The following equations denote the Jacobian matrices of the Barkley (B), Bär-Eiswirth 
(BE) and smooth Fenton-Karma (FK) model in this thesis. For the formalism of calculating Lyapunov exponents and vectors, whose application to RDEs is described in section 2.2.6. the first row of the Jacobian corresponds to $\mathrm{J}_{f}$ and the further rows to $\mathrm{J}_{H_{1}}$, $\mathrm{J}_{H_{2}}$ and so on.

$$
\begin{aligned}
\left.\mathrm{J}^{\mathrm{B}}\right|_{(u, v)} & =\left(\begin{array}{cc}
\left.\frac{1}{\epsilon}\left((1-2 u)\left(u-\frac{v+b}{a}\right)+u(1-u)\right)\right) & -\frac{1}{a \epsilon} u(1-u) \\
1 & -1
\end{array}\right) \\
\left.\mathrm{J}^{\mathrm{BE}}\right|_{(u, v)} & =\left(\begin{array}{cc}
\frac{1}{\epsilon}\left((1-2 u)\left(u-\frac{v+b}{a}\right)+u(1-u)\right) \\
\gamma(u) & -\frac{1}{a \epsilon} u(1-u) \\
-1
\end{array}\right),
\end{aligned}
$$

where

$$
\gamma(u)= \begin{cases}0, & u<\frac{1}{3} \\ -6.75(u-1)(3 u-1) & \frac{1}{3} \leq u \leq 1 \\ 0 & u \geq 1\end{cases}
$$

The Jacobian $J^{\mathrm{FK}}$ for the smooth Fenton-Karma model has been split up into its rows $\mathrm{J}_{1,\{1,2,3\}}^{\mathrm{FK}}, \mathrm{J}_{2,\{1,2,3\}}^{\mathrm{FK}}$ and $\mathrm{J}_{3,\{1,2,3\}}^{\mathrm{FK}}$ for better readability. As explained in section 2.1.6, within this thesis, the smooth approximation for the step function is $\Phi(x)=\Theta^{*}(x)=\frac{1}{2}(1+$ $\left.\tanh \left(k_{2} x\right)\right)$ resulting in $\Phi^{\prime}(x)=\frac{k_{2}}{2}\left(1-\tanh ^{2}\left(k_{2} x\right)\right)$.

$$
\begin{aligned}
& \left.\mathrm{J}_{1,\{1,2,3\}}^{\mathrm{FK}}\right|_{(u, v, w)}=\left[\begin{array}{c}
\frac{v}{\tau_{d}} \Phi^{\prime}\left(u-u_{c}\right)(1-u)\left(u-u_{c}\right)-\frac{v}{\tau_{d}} \Phi\left(u-u_{c}\right)\left(2 u-u_{c}-1\right) \\
-\frac{1}{\tau_{0}} \Phi\left(u_{c}-u\right)+\frac{u}{\tau_{0}} \Phi^{\prime}\left(u_{c}-u\right)-\frac{1}{\tau_{r}} \Phi^{\prime}\left(u-u_{c}\right) \\
\quad+\frac{w k}{2 \tau_{s i}}\left(1-\tanh ^{2}\left[k\left(u-u_{c}^{s i}\right)\right]\right), \\
\frac{1}{\tau_{d}} \Phi\left(u-u_{c}\right)(1-u)\left(u-u_{c}\right), \\
\frac{1}{2 \tau_{s i}}\left(1+\tanh \left[k\left(u-u_{c}^{s i}\right)\right]\right)
\end{array}\right] \\
& \left.J_{2,\{1,2,3\}}^{\mathrm{FK}}\right|_{(u, v, w)}=\left[\begin{array}{l}
\Phi^{\prime}\left(u_{c}-u\right)(1-v)\left(\frac{\Phi\left(u-u_{v}\right)}{\tau_{v 1}^{-}}+\frac{\Phi\left(u_{v}-u\right)}{\tau_{v 2}^{+}}\right) \\
+\Phi\left(u_{c}-u\right)(1-v)\left(\frac{\Phi^{\prime}\left(u-u_{v}\right)}{\tau_{v 1}^{-}}+\frac{\Phi^{\prime}\left(u_{v}-u\right)}{\tau_{v 2}^{+}}\right) \\
-\Phi^{\prime}\left(u-u_{c}\right) \frac{v}{\tau_{v}^{+}} \\
-\Phi\left(u_{c}-u\right)\left(\frac{\Phi\left(u-u_{v}\right)}{\tau_{v 1}^{-}}+\frac{\Phi\left(u_{v}-u\right)}{\tau_{v 2}^{+}}\right)-\Phi\left(u-u_{c}\right) \frac{1}{\tau_{v}^{+}} \\
0
\end{array}\right] \\
& \left.\mathrm{J}_{3,\{1,2,3\}}^{\mathrm{FK}}\right|_{(u, v, w)}=\left[\begin{array}{l}
-\Phi^{\prime}\left(u_{c}-u\right) \frac{1-w}{\tau_{w}^{-}}-\Phi^{\prime}\left(u-u_{c}\right) \frac{w}{\tau_{w}^{+}}, \\
0, \\
-\Phi\left(u_{c}-u\right) \frac{1}{\tau_{w}^{-}}-\Phi\left(u-u_{c}\right) \frac{1}{\tau_{w}^{+}}
\end{array}\right]
\end{aligned}
$$




\section{Appendix B}

\section{Supplementary data}

\begin{tabular}{cccc} 
Preparation & scaling exponent & Preparation & scaling exponent \\
\hline \hline$\# 1$ & $-0.7596 \pm 0.09$ & $\# 4$ & $-0.5441 \pm 0.05$ \\
$\# 1$ & $-0.7796 \pm 0.06$ & $\# 4$ & $-0.8050 \pm 0.12$ \\
$\# 2$ & $-0.3470 \pm 0.10$ & $\# 4$ & $-0.6658 \pm 0.07$ \\
$\# 2$ & $-0.9703 \pm 0.10$ & $\# 4$ & $-0.8288 \pm 0.09$ \\
$\# 3$ & $-0.9166 \pm 0.06$ & $\# 4$ & $-0.6345 \pm 0.03$ \\
$\# 3$ & $-0.5364 \pm 0.08$ & $\# 5$ & $-0.9839 \pm 0.07$ \\
$\# 3$ & $-0.9304 \pm 0.06$ & $\# 5$ & $-0.5293 \pm 0.09$ \\
$\# 3$ & $-0.6776 \pm 0.08$ & $\# 5$ & $-0.8614 \pm 0.10$ \\
$\# 3$ & $-0.8976 \pm 0.07$ & $\# 5$ & $-0.6705 \pm 0.09$ \\
$\# 3$ & $-0.5459 \pm 0.07$ & $\# 5$ & $-0.7938 \pm 0.08$ \\
$\# 4$ & $-0.6706 \pm 0.08$ & $\# 5$ & $-0.6405 \pm 0.10$
\end{tabular}

Table B.1: Scaling exponents for canine ventricular activation times $\tau(E)$.

\begin{tabular}{cccc} 
Preparation & scaling exponent & Preparation & scaling exponent \\
\hline \hline$\# 1$ & $-0.8035 \pm 0.06$ & $\# 2$ & $-1.1246 \pm 0.10$ \\
$\# 1$ & $-1.3799 \pm 0.09$ & $\# 3$ & $-0.8923 \pm 0.05$ \\
$\# 1$ & $-0.4462 \pm 0.11$ & $\# 3$ & $-0.7641 \pm 0.05$ \\
$\# 1$ & $-0.9767 \pm 0.10$ & $\# 3$ & $-0.7053 \pm 0.05$ \\
$\# 2$ & $-0.7745 \pm 0.06$ & $\# 3$ & $-0.7516 \pm 0.03$ \\
$\# 2$ & $-0.8091 \pm 0.05$ & $\# 3$ & $-0.5762 \pm 0.03$ \\
$\# 2$ & $-0.9509 \pm 0.08$ & $\# 3$ & $-0.5561 \pm 0.04$ \\
$\# 2$ & $-0.8043 \pm 0.08$ & $\# 3$ & $-0.5279 \pm 0.03$ \\
$\# 2$ & $-1.0111 \pm 0.08$ & &
\end{tabular}

Table B.2: Scaling exponents for canine atrial activation times $\tau(E)$. 


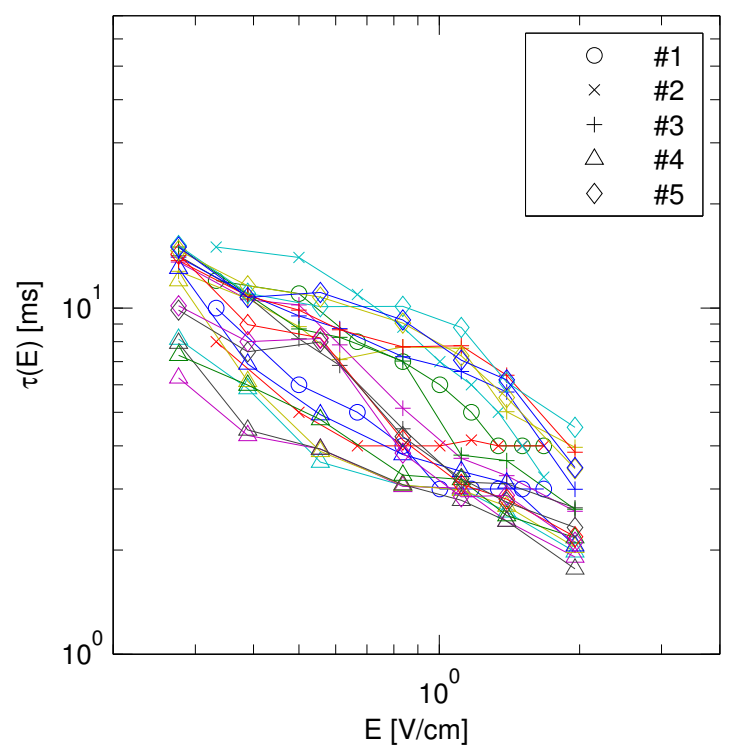

Figure B.1: Activation time observations $\tau(E)$ : canine ventricles.

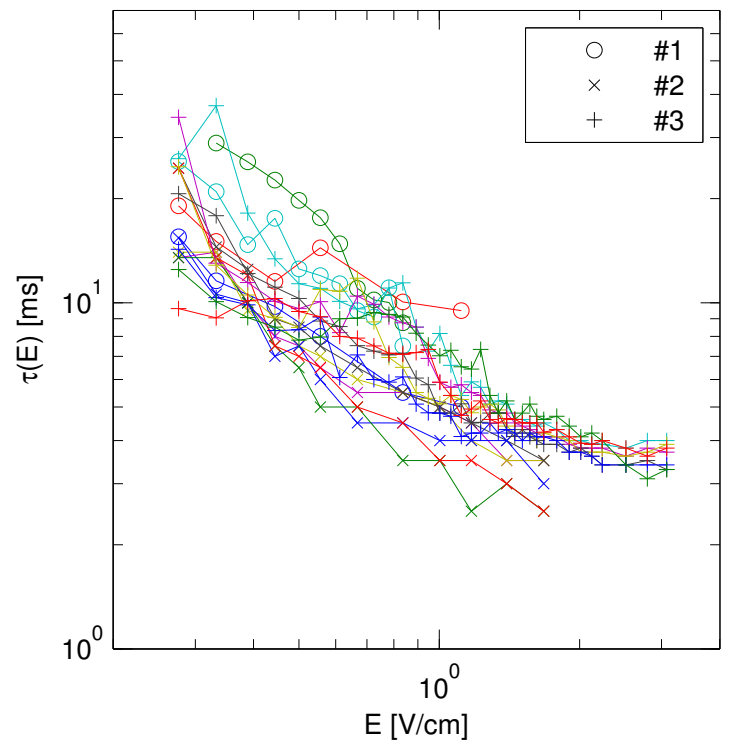

Figure B.2: Activation time observations $\tau(E)$ : canine atria. 
a)

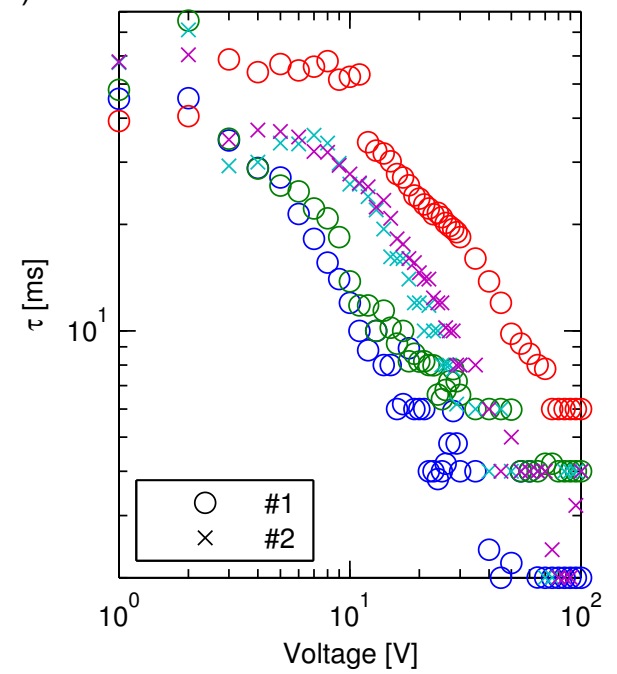

b)

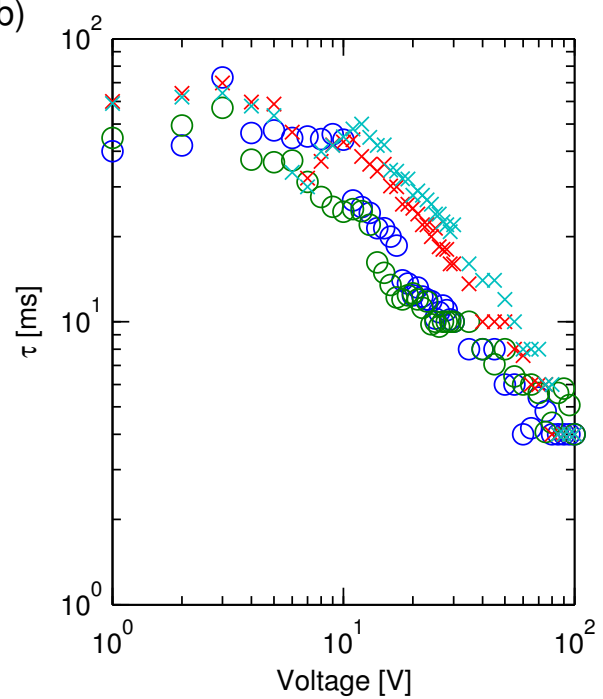

Figure B.3: Activation time observations in pig ventricular wedge preparations. a) Activation times observed for biphasic, rectangular electric-field pulses in two different preparations (marked by different symbols). b) Observations in the same preparations for truncated exponential (biphasic) pulses. 


\section{Appendix C}

\section{MediaSim - an open framework for simulating extended systems}

MediaSim is a simulation framework written entirely in $\mathrm{C}++$ [250], designed mainly for (but not restricted to) solving PDEs of the excitable-medium type using finite differences. It has a strictly object-oriented structure, is plugin-based for effortless extensibility and provides convenience functions for parallelizing operations on large arrays. The framework can be used either directly from a $\mathrm{C}++$ program, or through extensive MATLAB [187] and Python 251] interfaces.

MediaSim's plugins can be subdivided into five categories: Simulations, States, Initializers, Updaters and Analysers. Each category provides a superclass defining the interface for interaction with other components of the framework. Simulations coordinate the actions and relationships of all other components, States provide a container for the data Initializers, Updaters and Analysers work with. Using operator splitting techniques, the numerical algorithms for solving different terms of the PDE can be implemented in separate Updaters, which simplifies their optimization and enhances reusability of code for different numerical models.

Plugin implementations, i.e. classes derived from the above superclasses, can be fully customized without restrictions on external libraries or custom parallelization techniques while they can at the same time make use of convenience functions and objects provided by MediaSim. To facilitate interaction with numerical libraries, all array classes can be accessed as plain $\mathrm{C}$ arrays. As full-blown objects, they provide natural multi-index access similar to MATLAB or Python syntax, including subarray extraction and assignment realized through overloaded operators. All data objects offer conversion functions to MATLAB and NumPy [252] arrays. Components of the framework, including data objects, are serializable.

Apart from various local and global fixed-time and event-triggered stimulation terms, currently implemented Updaters include:

Local reaction models:

Barkley [72], Bär-Eiswirth [73], Bueno-Orovi et al. Minimal model [163], Fenton-Karma [84], Karma [101], Wang-Sobie [153] 
Diffusion operators:

Isotropic or anisotropic diffusion on rectangular Cartesian grids with no-flux or periodic boundary conditions (up to 3D), isotropic diffusion on polar grids with no-flux boundary conditions (2D), anisotropic diffusion on arbitrary geometries using the phase field method with no-flux boundary conditions [134] or special Neumann boundary conditions [218] (up to 3D), bi-domain model for isotropic or anisotropic spatial coupling [160]. 


\section{Index}

action potential duration

activation

activation map

anisotropy ratio

antitachycardia pacing

apparent velocity

apparent wave source

arrhythmia

atrial fibrillation

Bär-Eiswirth model

Barkley model

bi-domain equations

bottom-up approach

cable theory

cardiac arrest

conduction velocity

core conductor

Courant-Friedrichs-Lewy criterion

defibrillation

defibrillation threshold

Dirichlet boundary condition

dynamical heterogeneity

electrocardiogram

electrotonic constant

excitable medium

excitable system

excitation threshold

excitation-contraction coupling

Fenton-Karma model

smooth
46 fiber orientation

19

12 filament 49

75 finite differences 57

34 finite-element method 35

22 finite-volume method 35

75 forward Euler 58

75

21

21

45

43

30

42

30

21

47

30

64

forward-time centered-space

58

18

17

gating

29

gating variables

101

Gaussian random fields

17

Goldman-Hodgkin-Katz equation

55

Gram-Schmidt orthogonalization

implantable cardioverter-defibrillator 22

information dimension

106

initial value problem

57

inverted action potential

73

ion channels

17

144

ion homeostasis

16

23

latency

75

67 Lyapunov dimension

low-energy anti-fibrillation pacing

26

106

21

Lyapunov exponent

24

15

116

Lyapunov stability analysis

24

12

12

12

18

43

Lyapunov vectors

backward

55

covariant

56

forward

55

Gram-Schmidt 55

maximum principle

39

\section{3}

MediaSim

69

46 membrane voltage 
message-passing interface

mirco-computed tomography

mono-domain equation

motion artifact

Nernst equation

Neumann boundary condition

no-flux boundary condition

normal sinus rhythm

optical mapping

ordinary differential equation

partial differential equation

periodic boundary condition

phase singularities

phase-field method

potential

extracellular

intracellular

membrane

outside

potentiometric dye

probability density function

QR-decomposition

reaction-diffusion equation

refractory period
70 refractory tail

12

19 reversal potential

17

32 scroll wave

21

72 secondary sources

17 sheet

34 spiral tip

33 spiral wave

21 stencil

five-point

25

nine-point

62

31 stiff system

63

62 Stokes shift

47 structural heterogeneity

21

26 sudden cardiac death

tachycardia

top-down approach

43

16

topological charge

upstroke velocity

71 ventricular fibrillation

147 virtual electrodes

53 voltage-sensitive dye

57 von Neumann stability analysis

12 Wang-Sobie model 


\section{List of Figures}

1.1 Spatio-temporal patterns in different excitable media . . . . . . . . . . . . . 13

1.2 Anatomy of the heart . . . . . . . . . . . . . . . . . 14

1.3 Electrical conduction system of the heart $\ldots \ldots \ldots$. . . . . . . . . 16

1.4 Cardiac action potential . . . . . . . . . . . . . . . . . . 18

1.5 The tree of cardiac vessels $\ldots \ldots \ldots \ldots$. . . . . . . . . . . . . . . 20

1.6 Endocardial structure . . . . . . . . . . . . . . . . . . . . . . 20

2.1 Equivalent circuit diagram for the cell membrane . . . . . . . . . . . . 28

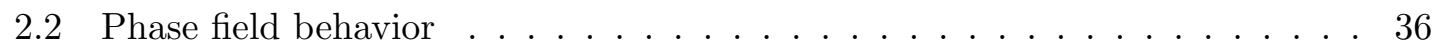

$2.3 \quad$ Volume integration at the phase-field interface $\ldots \ldots \ldots . \ldots . . \ldots 37$

2.4 Barkley model dynamics . . . . . . . . . . . . . . . . . . . . . 44

2.5 Barkley model: local phase space . . . . . . . . . . . . . . . . . 44

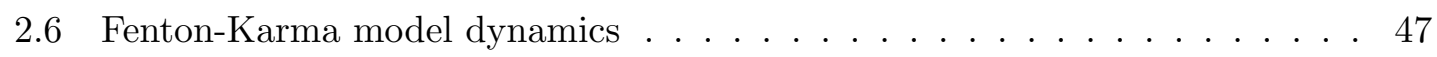

$2.7 \quad$ Spatial phase distribution $\ldots \ldots \ldots \ldots$. . . . . . . . . . . . . . . 48

2.8 Lyapunov stability analysis extracts growth rates of perturbations . . . . . 50

2.9 Numerical grid points in a one-dimensional domain . . . . . . . . . . . . 57

2.10 Optical mapping setup . . . . . . . . . . . . . . . . . . . . 72

2.11 Voltage-dependent excitation and emission spectra of di-4-ANEPPS . . . 73

2.12 Pulse shapes . . . . . . . . . . . . . . . . . . . . . . . . . 74

2.13 Signal processing of optical mapping data $\ldots \ldots \ldots$. . . . . . . . . . . . . . . . . . . 76

2.14 Activation map . . . . . . . . . . . . . . . . . . . . . . . 77

3.1 Example perturbations of a plane wave . . . . . . . . . . . . . . . 81

3.2 Wave front modulation for a plane wave . . . . . . . . . . . . . . . . 82

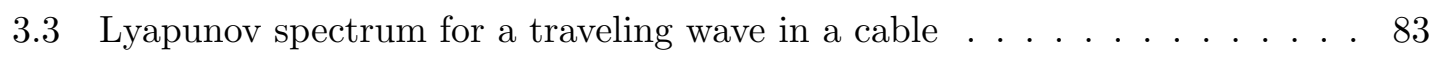

3.4 Lyapunov vectors of a rigidly rotating spiral wave . . . . . . . . . . . . . . . 88

$3.5 \quad$ Numerical artifacts in the Lyapunov spectrum (Barkley model) . . . . . . . 89

3.6 Numerical artifacts in the Lyapunov spectrum (Fenton-Karma model) . . . 90

3.7 Lyapunov exponents of multiple stable spirals . . . . . . . . . . . . . . . 93

3.8 Single-spiral perturbations in a system of multiple spirals . . . . . . . . . . . . . . 94

3.9 Bifurcation detection . . . . . . . . . . . . . . . . . . . . . . 95

3.10 Meandering modes for a rigidly rotating spiral . . . . . . . . . . . . . . 96

3.11 Lyapunov spectrum for different homogeneous excitation thresholds . . . . 98

3.12 Symmetry break by heterogeneity . . . . . . . . . . . . . . . . . . . . 99

3.13 Lyapunov vectors of a spiral wave interacting with a circular heterogeneity 101

3.14 Construction of random excitation threshold . . . . . . . . . . . . . . . 102 
3.15 Symmetry break by random heterogeneity . . . . . . . . . . . . . . . . . . 104

3.16 Lyapunov vectors of a spiral wave interacting with random heterogeneity . 105

3.17 Spatio-temporal chaos in the Bär-Eiswrith model . . . . . . . . . . . . . . 107

3.18 Spatio-temporal chaos in a heterogeneous system . . . . . . . . . . . . . 109

3.19 Complexity of chaos in a heterogeneous system . . . . . . . . . . . . 110

3.20 Spatio-temporal chaos in a heterogeneous system . . . . . . . . . . . . . 111

3.21 Spatio-temporal chaos in a heterogeneous system . . . . . . . . . . . . . . 112

3.22 Boundary effects in two-dimensional cardiac cell culture experiments . . . . 120

3.23 Increased susceptibility of convex boundaries . . . . . . . . . . . . . 121

3.24 Depolarization in a one-dimensional tissue domain . . . . . . . . . . . . . 122

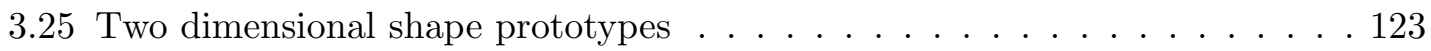

3.26 Membrane potential changes at circular boundaries . . . . . . . . . . . 125

3.27 Maximum depolarization for circular boundaries . . . . . . . . . . . . . . 127

3.28 Maximum depolarization for semi-circular protuberances . . . . . . . . . . 130

3.29 Maximum depolarization for parabolic boundaries . . . . . . . . . . . . . 131

3.30 Maximum depolarization for three-dimensional boundaries . . . . . . . . . 132

3.31 Summary: Depolarization for diffferent boundary types . . . . . . . . . . 133

3.32 Electric-field stimulation in a two-dimensional simulation . . . . . . . . . . 134

3.33 Electric-field stimulation in a three-dimensional simulation . . . . . . . . . . 136

3.34 Critical field strength for biphasic rectangular pulses: small curvatures . . . 138

3.35 Electric-field pulses of finite duration and different shapes at circular bound-

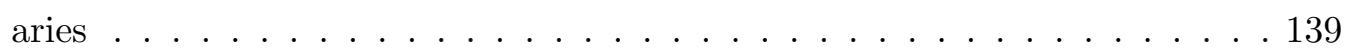

3.36 Time scale of depolarization at circular boundaries . . . . . . . . . . . . 140

3.37 Critical field strength for biphasic rectangular pulses: large curvatures . . . 142

3.38 Wave source recruitment model . . . . . . . . . . . . . . . . . . 148

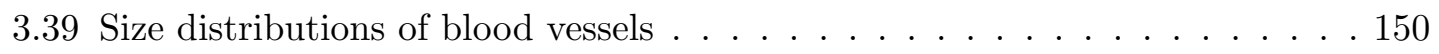

3.40 Tissue activation for different field strengths . . . . . . . . . . . . . . . . 151

3.41 Observed activation times . . . . . . . . . . . . . . . . . . 152

3.42 Averaged activation times . . . . . . . . . . . . . . . 153

3.43 Structure vs. function in canine ventricles . . . . . . . . . . . . . . 155

3.44 Structure vs. function in canine atria . . . . . . . . . . . . . . . . . 156

3.45 Size distribution estimation from canine activation times . . . . . . . . . . . . 158

3.46 Activation time scaling in pig ventricular preparations . . . . . . . . . . . 160

B.1 Activation time observations $\tau(E)$ : canine ventricles $\ldots \ldots \ldots$. . . . 180

B.2 Activation time observations $\tau(E)$ : canine atria . . . . . . . . . . . . . . 180

B.3 Activation time observations in pig ventricular wedge preparations . . . . . 181 


\section{List of Tables}

3.1 Lyapunov spectrum of a plane wave for complete coupling . . . . . . . . . . 85

3.2 Lyapunov spectrum of a plane wave for single-variable coupling . . . . . . . 86

3.3 Summary of binning parameters $\ldots \ldots \ldots \ldots \ldots$. . . . . . . . . 154

A.1 Parameter sets for the Barkley (B) and Bär-Eiswirth (BE) model . . . . . . 177

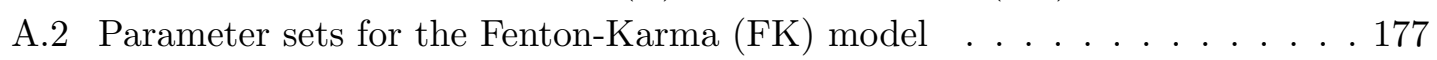

B.1 Scaling exponents for canine ventricular activation times $\tau(E)$. . . . . . 179

B.2 $\quad$ Scaling exponents for canine atrial activation times $\tau(E) \ldots \ldots$. . . . . 179 


\section{Bibliography}

[1] Dill, K. A. \& MacCallum, J. L. The protein-folding problem, 50 years on. Science 338, 1042-6 (2012).

[2] Kandt, C., Ash, W. L. \& Tieleman, D. P. Setting up and running molecular dynamics simulations of membrane proteins. Methods (San Diego, CA) 41, 475-88 (2007).

[3] Isin, B., Estiu, G., Wiest, O. \& Oltvai, Z. N. Identifying ligand binding conformations of the $\beta 2$-adrenergic receptor by using its agonists as computational probes. PLoS One 7, e50186 (2012).

[4] Guydosh, N. R. \& Block, S. M. Direct observation of the binding state of the kinesin head to the microtubule. Nature 461, 125-8 (2009).

[5] Prochniewicz, E., Guhathakurta, P. \& Thomas, D. D. The structural dynamics of actin during active interaction with myosin depends on the isoform of the essential light chain. Biochemistry 52, 1622-30 (2013).

[6] Diambra, L. \& Malta, C. P. Modeling the emergence of circadian rhythms in a clock neuron network. PLoS One 7, e33912 (2012).

[7] Mondragón-Palomino, O., Danino, T., Selimkhanov, J., Tsimring, L. \& Hasty, J. Entrainment of a population of synthetic genetic oscillators. Science 333, 1315-9 (2011).

[8] Palsson, E. \& Othmer, H. G. A model for individual and collective cell movement in dictyostelium discoideum. Proceedings of the National Academy of Sciences of the United States of America 97, 10448-53 (2000).

[9] Giuggioli, L., Pérez-Becker, S. \& Sanders, D. P. Encounter times in overlapping domains: Application to epidemic spread in a population of territorial animals. Physical Review Letters 110, 058103 (2013).

[10] van Oudenhove, L., Cerdá, X. \& Bernstein, C. An evolutionary dynamics model adapted to eusocial insects. PLoS One 8, e55159 (2013).

[11] Winfree, A. T. Biological rhythms and the behavior of populations of coupled oscillators. Journal of Theoretical Biology 16, 15-42 (1967).

[12] Walker, T. J. Acoustic synchrony: Two mechanisms in the snowy tree cricket. Science 166, 891-4 (1969).

[13] Jahnke, W., Skaggs, W. \& Winfree, A. Chemical vortex dynamics in the BelousovZhabotinsky reaction and in the 2-variable oregonator model. Journal of Physical Chemistry 93, 740-9 (1989).

[14] Winfree, A. Spiral waves of chemical activity. Science 175, 634-6 (1972). 
[15] Jakubith, S., Rotermund, H., Engel, W., Oertzen, A. V. \& Ertl, G. Spatiotemporal concentration patterns in a surface reaction: Propagating and standing waves, rotating spirals, and turbulence. Physical Review Letters 65, 3013-6 (1990).

[16] Tyson, J. J., Alexander, K. A., Manoranjan, V. \& Murray, J. Spiral waves of cyclic AMP in a model of slime mold aggregation. Physica D 34, 193-207 (1989).

[17] Huang, X. et al. Spiral waves in disinhibited mammalian neocortex. Journal of Neuroscience 24, 9897-902 (2004).

[18] Benninghoff, A. \& Drenckhahn, D. Anatomie, Band 2 (Elsevier GmbH, Urban \& Fischer Verlag, Munich, Germany, 2004), 16th edn.

[19] User: ZooFari. File: Heart diagram-en.svg URL http://commons.wikimedia. org/wiki/File:Heart_diagram-en.svg. Published under CC BY-SA 3.0: http://creativecommons.org/licenses/by-sa/3.0/deed.en; modified image may be reused under the same conditions.

[20] Schmidt, R. F., Lang, F. \& Thews, G. Physiologie des Menschen (Springer Medizin Verlag, Heidelberg, Germany, 2005), 29th edn.

[21] User: J. Heuser. File: Heart diagram-en.svg URL http://commons.wikimedia. org/wiki/File:RLS_12blauLeg.png. Published under Creative Commons Attribution 2.5 Generic License: http://creativecommons.org/licenses/by/2.5/; based on original work by Patrick J. Lynch, medical illustrator and C. Carl Jaffe, MD, cardiologist.

[22] Goldman, D. E. Potential, impedance, and rectification in membranes. Journal of General Physiology 27, 37-60 (1943).

[23] Hodgkin, A. L. \& Katz, B. The effect of sodium ions on the electrical activity of giant axon of the squid. Journal of Physiology 108, 37-77 (1949).

[24] Pickard, W. F. Generalizations of the Goldman-Hodgkin-Katz equation. Mathematical Biosciences 30, 99-111 (1976).

[25] Grant, A. O. Cardiac ion channels. Circulation: Arrhythmia and Electrophysiology 2, 185-94 (2009).

[26] Bers, D. M. Cardiac excitation-contraction coupling. Nature 415, 198-205 (2002).

[27] Bers, D. M. Calcium cycling and signaling in cardiac myocytes. Annual Review of Physiology 70, 23-49 (2008).

[28] Rohr, S. Role of gap junctions in the propagation of the cardiac action potential. Cardiovascular Research 62, 309-22 (2004).

[29] Sperelakis, N. An electric field mechanism for transmission of excitation between myocardial cells. Circulation Research 91, 985-7 (2002).

[30] Starmer, C. F. The role of intrinsic and induced vulnerability in electrically induced cardiac arrhythmias. Journal of Cardiovascular Electrophysiology 17, 1369-70 (2006).

[31] Kleber, A. G. The shape of the electrical action-potential upstroke: A new aspect from optical measurements on the surface of the heart. Circulation Research 97, 204-6 (2005). 
[32] Clayton, R. H. et al. Models of cardiac tissue electrophysiology: Progress, challenges and open questions. Progress in Biophysics and Molecular Biology 104, 22-48 (2011).

[33] Valderrábano, M. Influence of anisotropic conduction properties in the propagation of the cardiac action potential. Progress in Biophysics and Molecular Biology 94, 144-68 (2007).

[34] Antzelevitch, C. \& Fish, J. Electrical heterogeneity within the ventricular wall. Basic Research in Cardiology 96, 517-27 (2001).

[35] Szentadrassy, N. et al. Apico-basal inhomogeneity in distribution of ion channels in canine and human ventricular myocardium. Cardiovascular Research 65, 851-60 (2005).

[36] Kakkar, R. \& Lee, R. T. Intramyocardial fibroblast myocyte communication. Circulation Research 106, 47-57 (2010).

[37] Kohl, P., Camelliti, P., Burton, F. L. \& Smith, G. L. Electrical coupling of fibroblasts and myocytes: relevance for cardiac propagation. Journal of Electrocardiology 38, $45-50(2005)$.

[38] Winfree, A. Electrical instability in cardiac muscle: Phase singularities and rotors. Journal of Theoretical Biology 138, 353-405 (1989).

[39] Davidenko, J., Pertsov, A., Salomonsz, R., Baxter, W. \& Jalife, J. Stationary and drifting spiral waves of excitation in isolated cardiac muscle. Nature 355, 349-51 (1992).

[40] Pertsov, A., Davidenko, J., Salomonsz, R., Baxter, W. \& Jalife, J. Spiral waves of excitation underlie reentrant activity in isolated cardiac muscle. Circulation Research 72, 631-50 (1993).

[41] Krinsky, V. \& Agladze, K. Interaction of rotating waves in an active chemical medium. Physica D 8, 50-6 (1983).

[42] Gray, R., Pertsov, A. \& Jalife, J. Spatial and temporal organization during cardiac fibrillation. Nature 392, 75-8 (1998).

[43] Witkowski, F. et al. Spatiotemporal evolution of ventricular fibrillation. Nature 392, 78-82 (1998).

[44] Turakhia, M. \& Tseng, Z. H. Sudden cardiac death: Epidemiology, mechanisms, and therapy. Current Problems in Cardiology 32, 501-46 (2007).

[45] Wolf, P. A., Abbott, R. D. \& Kannel, W. B. Atrial fibrillation as an independent risk factor for stroke: The Framingham study. Stroke 22, 983-8 (1991).

[46] Ruan, Y., Liu, N. \& Priori, S. G. Sodium channel mutations and arrhythmias. Nature Reviews: Cardiology 6, 337-48 (2009).

[47] Thomas, N. L., Maxwell, C., Mukherjee, S. \& Williams, A. J. Ryanodine receptor mutations in arrhythmia: The continuing mystery of channel dysfunction. FEBS Letters 584, 2153-60 (2010).

[48] Lehnart, S. E. \& Lederer, W. J. An antidote for calcium leak: Targeting molecular arrhythmia mechanisms. Journal of Molecular and Cellular Cardiology 48, 279-82 (2010). 
[49] Schwartz, P. J., Billman, G. E. \& Stone, H. L. Autonomic mechanisms in ventricular fibrillation induced by myocardial ischemia during exercise in dogs with healed myocardial infarction. An experimental preparation for sudden cardiac death. Circulation 69, 790-800 (1984).

[50] Belevych, A. E. et al. Shortened Ca2+ signaling refractoriness underlies cellular arrhythmogenesis in a postinfarction model of sudden cardiac death. Circulation Research 110, 569-77 (2012).

[51] Wiggers, C. \& Wegria, R. Ventricular fibrillation due to single, localized induction and condenser shocks applied during the vulnerable phase of ventricular systole. American Journal of Physiology 128, 500-5 (1940).

[52] Oupadia, P. \& Ramaswamy, K. Images in clinical medicine. "R-on-T" phenomenon. The New England Journal of Medicine 338, 1812 (1998).

[53] McLeod, A. A. \& Jokhi, P. P. Pacemaker induced ventricular fibrillation in coronary care units. BMJ 328, 1249-50 (2004).

[54] Ng, G. A. Treating patients with ventricular ectopic beats. Heart 92, 1707-12 (2006).

[55] Wu, T. J. et al. Characteristics of wave fronts during ventricular fibrillation in human hearts with dilated cardiomyopathy: Role of increased fibrosis in the generation of reentry. Journal of the American College of Cardiology 32, 187-96 (1998).

[56] Spach, M. S. \& Boineau, J. P. Microfibrosis produces electrical load variations due to loss of side-to-side cell connections: A major mechanism of structural heart disease arrhythmias. Pacing and Clinical Electrophysiology 20, 397-413 (1997).

[57] Spach, M. S. Mounting evidence that fibrosis generates a major mechanism for atrial fibrillation. Circulation Research 101, 743-5 (2007).

[58] Tilz, R. R., Fedele, L., Satomi, K., Kuck, K. H. \& Antz, M. Idiopathic ventricular fibrillation. Herz 32, 233-9 (2007).

[59] Singla, S., Karam, P., Deshmukh, A. J., Mehta, J. \& Paydak, H. Review of contemporary antiarrhythmic drug therapy for maintenance of sinus rhythm in atrial fibrillation. Journal of Cardiovascular Pharmacology and Therapeutics 17, 12-20 (2012).

[60] Piccini, J. P. et al. Antiarrhythmic drug therapy for sustained ventricular arrhythmias complicating acute myocardial infarction. Critical Care Medicine 39, 78-83 (2011).

[61] Chowdhury, P., Lewis, W. R., Schweikert, R. A. \& Cummings, J. E. Ablation of atrial fibrillation: What can we tell our patients? Cleveland Clinic Journal of Medicine 76, 543-50 (2009).

[62] Terasawa, T. et al. Comparative effectiveness of radiofrequency catheter ablation for atrial fibrillation. Annals of Internal Medicine 151, 191-202 (2009).

[63] Tung, R., Boyle, N. G. \& Shivkumar, K. Catheter ablation of ventricular tachycardia. Circulation 122, e389-91 (2010).

[64] Rosman, J., Hoffmeister, P., Reynolds, M. \& Peralta, A. Possible proarrhythmia with dronedarone. Journal of Cardiovascular Electrophysiology 24, 103-4 (2013). 
[65] Roden, D. M. Mechanisms and management of proarrhythmia. The American Journal of Cardiology 82, 49I-57I (1998).

[66] Robinson, T. \& Kalman, J. M. Proarrhythmia following prior pulmonary vein isolation: What is the mechanism? Journal of Cardiovascular Electrophysiology 23, 884-6 (2011).

[67] Hilleman, D. E. \& Bauman, A. L. Role of antiarrhythmic therapy in patients at risk for sudden cardiac death: An evidence-based review. Pharmacotherapy 21, 556-75 (2001).

[68] Schwab, J. O. Antitachycardia pacing to terminate ventricular tachyarrhythmia: New insights into how to reduce painful implantable cardioverter defibrillator shocks. Europace 14, 1381-2 (2012).

[69] Tung, L., Tovar, O., Neunlist, M., Jain, S. K. \& O'Neill, R. J. Effects of strong electrical shock on cardiac muscle tissue. Annals of the New York Academy of Sciences 720, 160-75 (1994).

[70] Walcott, G., Killingsworth, C. \& Ideker, R. Do clinically relevant transthoracic defibrillation energies cause myocardial damage and dysfunction? Resuscitation 59, 59-70 (2003).

[71] Matchett, M. et al. The implantable cardioverter defibrillator: Its history, current psychological impact and future. Expert Review of Medical Devices 6, 43-50 (2009).

[72] Barkley, D., Kness, M. \& Tuckerman, L. Spiral-wave dynamics in a simple model of excitable media: The transition from simple to compound rotation. Physical Review A 42, 2489-92 (1990).

[73] Bär, M. \& Eiswirth, M. Turbulence due to spiral breakup in a continuous excitable medium. Physical Review E 48, R1635-7 (1993).

[74] Bub, G., Shrier, A. \& Glass, L. Spiral wave generation in heterogeneous excitable media. Physical Review Letters 88, 058101 (2002).

[75] Bub, G., Shrier, A. \& Glass, L. Global organization of dynamics in oscillatory heterogeneous excitable media. Physical Review Letters 94, 028105 (2005).

[76] Steinberg, B., Glass, L., Shrier, A. \& Bub, G. The role of heterogeneities and intercellular coupling in wave propagation in cardiac tissue. Philosophical Transactions of the Royal Society A: Mathematical, Physical and Engineering Sciences 364, 1299-1311 (2006).

[77] Sampson, K. J. \& Henriquez, C. S. Interplay of ionic and structural heterogeneity on functional action potential duration gradients: Implications for arrhythmogenesis. Chaos 12, 819-28 (2002).

[78] Zou, X., Levine, H. \& Kessler, D. Interaction between a drifting spiral and defects. Physical Review E 47, R800-3 (1993).

[79] Zemlin, C. W. \& Pertsov, A. M. Anchoring of drifting spiral and scroll waves to impermeable inclusions in excitable media. Physical Review Letters 109, 038303 (2012).

[80] Lim, Z. Y., Maskara, B., Aguel, F., Emokpae, R. \& Tung, L. Spiral wave attachment to millimeter-sized obstacles. Circulation 114, 2113-21 (2006). 
[81] Biktasheva, I. V., Holden, A. V. \& Biktashev, V. N. Localization of response functions of spiral waves in the FitzHugh-Nagumo system. International Journal of Bifurcation and Chaos 16, 1547-55 (2006).

[82] Sridhar, S., Sinha, S. \& Panfilov, A. V. Anomalous drift of spiral waves in heterogeneous excitable media. Physical Review E 82, 051908 (2010).

[83] Sadeghi, P. \& Rotermund, H. H. Gradient induced spiral drift in heterogeneous excitable media. Chaos 21, 013125 (2011).

[84] Fenton, F. H. \& Karma, A. Vortex dynamics in three-dimensional continuous myocardium with fiber rotation: Filament instability and fibrillation. Chaos 8, 20-47 (1998).

[85] Rappel, W.-J. Filament instability and rotational tissue anisotropy: A numerical study using detailed cardiac models. Chaos 11, 71-80 (2001).

[86] Dierckx, H., Bernus, O. \& Verschelde, H. A geometric theory for scroll wave filaments in anisotropic excitable media. Physica D 238, 941-50 (2009).

[87] Plonsey, R. \& Barr, R. C. Effect of microscopic and macroscopic discontinuities on the response of cardiac tissue to defibrillating (stimulating) currents. Medical $\mathbb{E}$ Biological Engineering \& Computing 24, 130-6 (1986).

[88] Plonsey, R. \& Barr, R. C. Inclusion of junction elements in a linear cardiac model through secondary sources: Application to defibrillation. Medical $\& 3$ Biological Engineering $\&$ Computing 24, 137-44 (1986).

[89] Krassowska, W., Pilkington, T. C. \& Ideker, R. E. Periodic conductivity as a mechanism for cardiac stimulation and defibrillation. IEEE Transactions on Biomedical Engineering 34, 555-60 (1987).

[90] Plonsey, R., Barr, R. C. \& Witkowski, F. X. One-dimensional model of cardiac defibrillation. Medical \& Biological Engineering \& Computing 29, 465-9 (1991).

[91] Gillis, A. M., Fast, V. G., Rohr, S. \& Kléber, A. G. Spatial changes in transmembrane potential during extracellular electrical shocks in cultured monolayers of neonatal rat ventricular myocytes. Circulation Research 79, 676-90 (1996).

[92] Sobie, E., Susil, R. \& Tung, L. A generalized activating function for predicting virtual electrodes in cardiac tissue. Biophysical Journal 73, 1410-23 (1997).

[93] Fishler, M. \& Vepa, K. Spatiotemporal effects of syncytial heterogeneities on cardiac far-field excitations during monophasic and biphasic shocks. Journal of Cardiovascular Electrophysiology 9, 1310-24 (1998).

[94] Fishler, M. Syncytial heterogeneity as a mechanism underlying cardiac far-field stimulation during defibrillation-level shocks. Journal of Cardiovascular Electrophysiology 9, 384-94 (1998).

[95] Trayanova, N., Skouibine, K. \& Moore, P. Virtual electrode effects in defibrillation. Progress in Biophysics and Molecular Biology 69, 387-403 (1998).

[96] Basser, P. J. \& Roth, B. J. New currents in electrical stimulation of excitable tissues. Annual Review of Biomedical Engineering 2, 377-97 (2000). 
[97] Trayanova, N., Gray, R., Bourn, D. \& Eason, J. Virtual electrode-induced positive and negative graded responses: New insights into fibrillation induction and defibrillation. Journal of Cardiovascular Electrophysiology 14, 756-63 (2003).

[98] Efimov, I. \& Ripplinger, C. M. Virtual electrode hypothesis of defibrilation. Heart Rhythm 3, 1100-2 (2006).

[99] Trayanova, N. Concepts of ventricular defibrillation. Philosophical Transactions of the Royal Society A: Mathematical, Physical and Engineering Sciences 359, 1327-37 (2001).

[100] Dosdall, D. J., Fast, V. G. \& Ideker, R. E. Mechanisms of defibrillation. Annual Review of Biomedical Engineering 12, 233-58 (2010).

[101] Karma, A. Spiral breakup in model equations of action potential propagation in cardiac tissue. Physical Review Letters 71, 1103-6 (1993).

[102] Qu, Z., Xie, F., Garfinkel, A. \& Weiss, J. N. Origins of spiral wave meander and breakup in a two-dimensional cardiac tissue model. Annals of Biomedical Engineering 28, 755-71 (2000).

[103] Panfilov, A. V. Spiral breakup in an array of coupled cells: The role of the intercellular conductance. Physical Review Letters 88, 118101 (2002).

[104] Fenton, F. H., Cherry, E. M., Hastings, H. \& Evans, S. Multiple mechanisms of spiral wave breakup in a model of cardiac electrical activity. Chaos 12, 852-92 (2002).

[105] Otani, N. F. et al. Characterization of multiple spiral wave dynamics as a stochastic predator-prey system. Physical Review E 78, 021913 (2008).

[106] Barkley, D. Linear stability analysis of rotating spiral waves in excitable media. Physical Review Letters 68, 2090-3 (1992).

[107] Barkley, D. Euclidean symmetry and the dynamics of rotating spiral waves. Physical Review Letters 72, 164-7 (1994).

[108] Allexandre, D. \& Otani, N. F. Preventing alternans-induced spiral wave breakup in cardiac tissue: An ion-channel-based approach. Physical Review E 70, 061903 (2004).

[109] Biktasheva, I. V., Barkley, D., Biktashev, V. N. \& Foulkes, A. J. Computation of the drift velocity of spiral waves using response functions. Physical Review E 81, 066202 (2010).

[110] Lyapunov, A. M. The general problem of the stability of motion (1892). PhD Thesis.

[111] Oseledec, V. I. A multiplicative ergodic theorem. Ljapunov characteristic numbers for dynamical systems. Transaction of the Moscow Mathematical Society 19, 197231 (1968).

[112] Benettin, G., Galgani, L., Giorgilli, A. \& Strelcyn, J.-M. Lyapunov characteristic exponents for smooth dynamical systems and for hamiltonian systems; a method for computing all of them. Part 1: Theory. Meccanica 15, 9-20 (1980).

[113] Benettin, G., Galgani, L., Giorgilli, A. \& Strelcyn, J.-M. Lyapunov characteristic exponents for smooth dynamical systems and for hamiltonian systems; a method for computing all of them. Part 2: Numerical application. Meccanica 15, 21-30 (1980). 
[114] Ginelli, F. et al. Characterizing dynamics with covariant Lyapunov vectors. Physical Review Letters 99, 130601 (2007).

[115] Szendro, I. G., Pazo, D., Rodriguez, M. A. \& Lopez, J. M. Spatiotemporal structure of Lyapunov vectors in chaotic coupled-map lattices. Physical Review E 76, 025202 (2007).

[116] Pazo, D., Szendro, I. G., Lopez, J. M. \& Rodriguez, M. A. Structure of characteristic Lyapunov vectors in spatiotemporal chaos. Physical Review E 78, 016209 (2008).

[117] Yang, H., Takeuchi, K. A., Ginelli, F., Chate, H. \& Radons, G. Hyperbolicity and the effective dimension of spatially extended dissipative systems. Physical Review Letters 102, 074102 (2009).

[118] Kuptsov, P. V. \& Parlitz, U. Theory and computation of covariant Lyapunov vectors. Journal of Nonlinear Science 22, 727-62 (2012).

[119] Hoover, W. \& Hoover, C. G. Local Gram-Schmidt and covariant Lyapunov vectors and exponents for three harmonic oscillator problems. Communications in Nonlinear Science and Numerical Simulation 17, 1043-54 (2012).

[120] Qu, Z., Weiss, J. N. \& Garfinkel, A. From local to global spatiotemporal chaos in a cardiac tissue model. Physical Review E 61, 727-32 (2000).

[121] Strain, M. \& Greenside, H. Size-dependent transition to high-dimensional chaotic dynamics in a two-dimensional excitable medium. Physical Review Letters 80, 23069 (1998).

[122] Trayanova, N., Skouibine, K. \& Aguel, F. The role of cardiac tissue structure in defibrillation. Chaos 8, 221-33 (1998).

[123] Trayanova, N. \& Skouibine, K. Modeling defibrillation: Effects of fiber curvature. Journal of Electrocardiology 31, 23-9 (1998).

[124] Hooks, D. et al. Cardiac microstructure: Implications for electrical, propagation and defibrillation in the heart. Circulation Research 91, 331-8 (2002).

[125] Entcheva, E. et al. Virtual electrode effects in transvenous defibrillation-modulation by structure and interface: Evidence from bidomain simulations and optical mapping. Journal of Cardiovascular Electrophysiology 9, 949-61 (1998).

[126] Entcheva, E., Trayanova, N. \& Claydon, F. Patterns of and mechanisms for shockinduced polarization in the heart: A bidomain analysis. IEEE Transactions on Biomedical Engineering 46, 260-70 (1999).

[127] Pumir, A. \& Krinsky, V. I. Unpinning of a rotating wave in cardiac muscle by an electric field. Journal of Theoretical Biology 199, 311-9 (1999).

[128] Takagi, S. et al. Unpinning and removal of a rotating wave in cardiac muscle. Physical Review Letters 93, 058101 (2004).

[129] Ripplinger, C. M., Krinsky, V. I., Nikolski, V. P. \& Efimov, I. R. Mechanisms of unpinning and termination of ventricular tachycardia. American Journal of Physiology: Heart and Circulatory Physiology 291, H184-92 (2006).

[130] Pumir, A. et al. Wave emission from heterogeneities opens a way to controlling chaos in the heart. Physical Review Letters 99, 208101 (2007). 
[131] Bittihn, P. et al. Far field pacing supersedes anti-tachycardia pacing in a generic model of excitable media. New Journal of Physics 10, 103012 (2008).

[132] Bittihn, P. et al. Phase-resolved analysis of the susceptibility of pinned spiral waves to far-field pacing in a two-dimensional model of excitable media. Philosophical Transactions of the Royal Society A: Mathematical, Physical and Engineering Sciences 368, 2221-36 (2010).

[133] Hörning, M., Isomura, A., Jia, Z., Entcheva, E. \& Yoshikawa, K. Utilizing the eikonal relationship in strategies for reentrant wave termination in excitable media. Physical Review E 81, 056202 (2010).

[134] Fenton, F. H., Cherry, E. M., Karma, A. \& Rappel, W. Modeling wave propagation in realistic heart geometries using the phase-field method. Chaos 15, 013502 (2005).

[135] Fenton, F. H. et al. Termination of atrial fibrillation using pulsed low-energy far-field stimulation. Circulation 120, 467-4 (2009).

[136] Li, W. et al. Low-energy multistage atrial defibrillation therapy terminates atrial fibrillation with less energy than a single shock. Circulation: Arrhythmia and Electrophysiology 4, 917-25 (2011).

[137] Ambrosi, C. M., Ripplinger, C. M., Efimov, I. R. \& Fedorov, V. V. Termination of sustained atrial flutter and fibrillation using low-voltage multiple-shock therapy. Heart Rhythm 8, 101-8 (2011).

[138] Allessie, M., Kirchhof, C., Scheffer, G., Chorro, F. \& Brugada, J. Regional control of atrial fibrillation by rapid pacing in conscious dogs. Circulation 84, 1689-97 (1991).

[139] Hodgkin, A. L. \& Huxley, A. F. A quantitative description of membrane current and its application to conduction and excitation in nerve. Journal of Physiology 117, 500-44 (1952).

[140] Hodgkin, A. L., Huxley, A. F. \& Katz, B. Measurement of current-voltage relations in the membrane of the giant axon of Loligo. Journal of Physiology 116, 424-48 (1952).

[141] Hodgkin, A. L. \& Huxley, A. F. The dual effect of membrane potential on sodium conductance in the giant axon of Loligo. Journal of Physiology 116, 497-506 (1952).

[142] Hodgkin, A. L. \& Huxley, A. F. Currents carried by sodium and potassium ions through the membrane of the giant axon of Loligo. Journal of Physiology 116, 449-72 (1952).

[143] Hodgkin, A. L. \& Huxley, A. F. The components of membrane conductance in the giant axon of Loligo. Journal of Physiology 116, 473-96 (1952).

[144] Cole, K. Dynamic electrical characteristics of the squid axon membrane. Archives des Sciences Physiologiques 3, 253-8 (1949).

[145] Marmont, G. Studies on the axon membrane: I. A new method. Journal of Cellular Physiology 34, 351-82 (1949).

[146] Noble, D. A modification of the Hodgkin-Huxley equations applicable to Purkinje fibre action and pace-maker potentials. Journal of Physiology 160, 317-52 (1962).

[147] Noble, D. Applications of Hodgkin-Huxley equations to excitable tissues. Physiological Reviews 46, 1-50 (1966). 
[148] Zhao, Y. et al. Patch clamp technique: Review of the current state of the art and potential contributions from nanoengineering. Proceedings of the Institution of Mechanical Engineers, Part N: Journal of Nanoengineering and Nanosystems 222, $1-11(2009)$.

[149] Tammaro, P., Shimomura, K. \& Proks, P. Xenopus oocytes as a heterologous expression system for studying ion channels with the patch-clamp technique. Methods in Molecular Biology (Clifton, NJ) 491, 127-39 (2008).

[150] Heimburg, T. \& Jackson, A. D. On soliton propagation in biomembranes and nerves. Proceedings of the National Academy of Sciences of the United States of America 102, 9790-5 (2005).

[151] Heimburg, T. \& Jackson, A. D. On the action potential as a propagating density pulse and the role of anesthetics. Biophysical Reviews and Letters 02, 57-78 (2007).

[152] Heimburg, T. The capacitance and electromechanical coupling of lipid membranes close to transitions: The effect of electrostriction. Biophysical Journal 103, 918-29 (2012).

[153] Wang, L. J. \& Sobie, E. A. Mathematical model of the neonatal mouse ventricular action potential. American Journal of Physiology: Heart and Circulatory Physiology 294, H2565-75 (2008).

[154] Fox, R. F. Stochastic versions of the Hodgkin-Huxley equations. Biophysical Journal 72, 2068-74 (1997).

[155] Goldwyn, J. H., Imennov, N. S., Famulare, M. \& Shea-Brown, E. Stochastic differential equation models for ion channel noise in Hodgkin-Huxley neurons. Physical Review E 83, 041908 (2011).

[156] Clark, J. \& Plonsey, R. A mathematical evaluation of the core conductor model. Biophysical Journal 6, 95-112 (1966).

[157] Spach, M. S. \& Barr, R. C. Origin of epicardial ST-T wave potentials in the intact dog. Circulation Research 39, 475-87 (1976).

[158] Miller, W. T. \& Geselowitz, D. B. Simulation studies of the electrocardiogram. I. The normal heart. Circulation Research 43, 301-15 (1978).

[159] Spach, M. S., Miller, W. T., Miller-Jones, E., Warren, R. B. \& Barr, R. C. Extracellular potentials related to intracellular action potentials during impulse conduction in anisotropic canine cardiac muscle. Circulation Research 45, 188-204 (1979).

[160] Roth, B. \& Wikswo, J. A bidomain model for the extracellular potential and magnetic field of cardiac tissue. IEEE Transactions on Biomedical Engineering 33, 467-9 (1986).

[161] Roth, B. Action potential propagation in a thick strand of cardiac muscle. Circulation Research 68, 162-73 (1991).

[162] Bueno-Orovio, A., Pérez-García, V. M. \& Fenton, F. H. Spectral methods for partial differential equations in irregular domains: The spectral smoothed boundary method. SIAM Journal on Scientific Computing 28, 886-900 (2006).

[163] Bueno-Orovio, A., Cherry, E. M. \& Fenton, F. H. Minimal model for human ventricular action potentials in tissue. Journal of Theoretical Biology 253, 544-60 (2008). 
[164] Li, X., Lowengrub, J., Rätz, A. \& Voigt, A. Solving PDEs in complex geometries: A diffuse domain approach. Communications in Mathematical Sciences 7, 81-107 (2009).

[165] Baudoin, F. Diffusion semi-group lectures (Wordpress.com, 2012). URL http://fabricebaudoin.wordpress.com/2012/03/28/ lecture-3-the-heat-equation-associated-to-a-diffusion-operator/. Lecture 3: The heat equation associated to a diffusion operator.

[166] Fenton, F. H. \& Cherry, E. M. Models of cardiac cell. Scholarpedia 3, 1868 (2008). URL http://www.scholarpedia.org/article/Models_of_cardiac_cell.

[167] Barkley, D. A model for fast computer simulation of waves in excitable media. Physica D 49, 61-70 (1991).

[168] FitzHugh, R. Impulses and physiological states in theoretical models of nerve membrane. Biophysical Journal 1, 445-66 (1961).

[169] Nagumo, J., Arimoto, S. \& Yoshizawa, S. An active pulse transmission line simulating nerve axon. Proceedings of the IRE 50, 2061-70 (1962).

[170] Iyer, A. \& Gray, R. An experimentalist's approach to accurate localization of phase singularities during reentry. Annals of Biomedical Engineering 29, 47-59 (2001).

[171] Bray, M., Lin, S.-F., Aliev, R., Roth, B. \& Wikswo, J. Experimental and theoretical analysis of phase singularity dynamics in cardiac tissue. Journal of Cardiovascular Electrophysiology 12, 716-22 (2001).

[172] Clayton, R., Zhuchkova, E. \& Panfilov, A. Phase singularities and filaments: Simplifying complexity in computational models of ventricular fibrillation. Progress in Biophysics and Molecular Biology 90, 378-98 (2006).

[173] Mermin, N. Topological theory of defects in ordered media. Reviews of Modern Physics 51, 591-648 (1979).

[174] Ruelle, D. Analycity properties of the characteristic exponents of random matrix products. Advances in Mathematics 32, 68-80 (1979).

[175] Ruelle, D. Ergodic theory of differentiable dynamical systems. Publications Mathématiques de l'IHÉS 50, 27-58 (1979).

[176] Raghunathan, M. S. A proof of Oseledec's multiplicative ergodic theorem. Israel Journal of Mathematics 32, 356-62 (1979).

[177] Eckmann, J. \& Ruelle, D. Ergodic theory of chaos and strange attractors. Reviews of Modern Physics 57, 617-56 (1985).

[178] Wolfe, C. L. \& Samelson, R. M. An efficient method for recovering Lyapunov vectors from singular vectors. Tellus Series A: Dynamic Meteorology and Oceanography 59, 355-66 (2007).

[179] Strikwerda, J. Finite difference schemes and partial differential equations (Society for Industrial and Applied Mathematics (SIAM), Philadelphia, PA, USA, 2004), 2nd edn. URL http://books.google.de/books?id=SH8R_flZBGIC.

[180] Press, W. H., Teukolsky, S. A., Vetterling, W. T. \& Flannery, B. P. Numerical recipes in C: The art of scientific computing (Cambridge University Press, New York, NY, USA, 1992), 2nd edn. 
[181] Cherry, E. M., Greenside, H. S. \& Henriquez, C. S. Efficient simulation of threedimensional anisotropic cardiac tissue using an adaptive mesh refinement method. Chaos 13, 853-65 (2003).

[182] Hornung, D. Cardiac arrhythmia termination on the vascular and organ scale (University of Göttingen, 2013). PhD Thesis. In preparation.

[183] Behrend, A. Interaction of pinned spiral waves with a pulsed electric field in heterogeneous excitable media (University of Göttingen, 2011). Diploma Thesis.

[184] Linke, P. Segmentation of a human whole heart scan and simulation of the cardiac electrical conduction system (University of Göttingen, 2012). Bachelor's Thesis.

[185] Mayer, A. Spatio-temporal dynamics in a numerical model for ventricular tissue of neonatal mice (University of Göttingen, 2012). Bachelor's Thesis.

[186] tom Wörden, H. Simulation of the electrical activity of heart tissue using the bidomain model (University of Göttingen, 2012). Bachelor's Thesis.

[187] MATLAB (The MathWorks Inc., Natick, Massachusetts, USA). URL http://www . mathworks.com/

[188] Cohen, L. B., Keynes, R. D. \& Hille, B. Light scattering and birefringence changes during nerve activity. Nature 218, 438-41 (1968).

[189] Tasaki, I., Watanabe, A., Sandlin, R. \& Carnay, L. Changes in fluorescence, turbidity, and birefringence associated with nerve excitation. Proceedings of the National Academy of Sciences of the United States of America 61, 883-8 (1968).

[190] Salzberg, B. M., Davila, H. V. \& Cohen, L. B. Optical recording of impulses in individual neurones of an invertebrate central nervous system. Nature 246, 508-9 (1973).

[191] Salama, G. \& Morad, M. Merocyanine 540 as an optical probe of transmembrane electrical activity in the heart. Science 191, 485-7 (1976).

[192] Salama, G. Optical probes of membrane potential in heart muscle. Journal of Physiology 292, 267-95 (1979).

[193] Loew, L. M. Design and characterization of electrochromic membrane probes. Journal of Biochemical and Biophysical Methods 6, 243-60 (1982).

[194] Fluhler, E., Burnham, V. G. \& Loew, L. M. Spectra, membrane binding, and potentiometric responses of new charge shift probes. Biochemistry 24, 5749-55 (1985).

[195] Montana, V. A naphthyl analog of the aminostyryl pyridinium class of potentiometric membrane dyes shows consistent sensitivity in a variety of tissue, cell, and model membrane preparations. Journal of Membrane Biology 130, 1-10 (1992).

[196] Salama, G. et al. Properties of new, long-wavelength, voltage-sensitive dyes in the heart. Journal of Membrane Biology 208, 125-40 (2005).

[197] Matiukas, A. New near-infrared optical probes of cardiac electrical activity. American Journal of Physiology: Heart and Circulatory Physiology 290, H2633-43 (2006).

[198] Efimov, I. R. Optical imaging of the heart. Circulation Research 95, 21-33 (2004). 
[199] Efimov, I. \& Salama, G. The future of optical mapping is bright: RE: Review on: "Optical imaging of voltage and calcium in cardiac cells and tissues" by Herron, Lee, and Jalife. Circulation Research 110, e70-1 (2012).

[200] Herron, T. J., Lee, P. \& Jalife, J. Optical imaging of voltage and calcium in cardiac cells \& tissues. Circulation Research 110, 609-23 (2012).

[201] Luther, S. et al. Low-energy control of electrical turbulence in the heart. Nature 475, 235-9 (2011).

[202] Fedorov, V. V. et al. Application of blebbistatin as an excitation-contraction uncoupler for electrophysiologic study of rat and rabbit hearts. Heart Rhythm 4, 619-26 (2007).

[203] Bachtel, A. D. et al. A novel approach to dual excitation ratiometric optical mapping of cardiac action potentials with di-4-ANEPPS using pulsed led excitation. IEEE Transactions on Biomedical Engineering 58, 2120-6 (2011).

[204] Morley, G. et al. Characterization of conduction in the ventricles of normal and heterozygous cx43 knockout mice using optical mapping. Journal of Cardiovascular Electrophysiology 10, 1361-75 (1999).

[205] Nygren, A. et al. Voltage-sensitive dye mapping of activation and conduction in adult mouse hearts. Annals of Biomedical Engineering 28, 958-67 (2000).

[206] Mourad, A. \& Nash, M. P. Method for quantifiying conduction velocity during ventricular fibrillation. Physical Review E 75, 011914 (2007).

[207] Petitprez, S. et al. SAP97 and dystrophin macromolecular complexes determine two pools of cardiac sodium channels Nav1.5 in cardiomyocytes. Circulation Research 108, 294-304 (2010).

[208] Zemlin, C., Mironov, S. \& Pertsov, A. Near-threshold field stimulation: Intramural versus surface activation. Cardiovascular Research 69, 98-106 (2006).

[209] Karma, A. Physics of cardiac arrhythmogenesis. Annual Review of Condensed Matter Physics 4, 313-37 (2013).

[210] Bochi, J. \& Viana, M. The Lyapunov exponents of generic volume-preserving and symplectic maps. Annals of Mathematics 161, 1423-85 (2005).

[211] Pugh, C., Shub, M. \& Starkov, A. Stable ergodicity. Bulletin of the American Mathematical Society 41, 1-41 (2004).

[212] Heagy, J., Carroll, T. \& Pecora, L. Synchronous chaos in coupled oscillator systems. Physical Review E 50, 1874-85 (1994).

[213] Panfilov, A. V. \& Keener, J. Re-entry in three-dimensional FitzHugh-Nagumo medium with rotational anisotropy. Physica D 84, 545-52 (1995).

[214] Panfilov, A. V. \& Vasiev, B. Vortex initiation in a heterogeneous excitable medium. Physica D 49, 107-13 (1991).

[215] ten Tusscher, K. H. W. J. \& Panfilov, A. V. Reentry in heterogeneous cardiac tissue described by the Luo-Rudy ventricular action potential model. American Journal of Physiology: Heart and Circulatory Physiology 284, H542-8 (2003). 
[216] Kaplan, J. L. \& Yorke, J. A. Chaotic behavior of multidimensional difference equations. In Peitgen, H. O. \& Walther, H. O. (eds.) Functional differential equations and approximations of fixed points, Lecture notes in Mathematics, 730, 204 (Springer, Berlin, Germany, 1979).

[217] Rényi, A. On the dimension and entropy of probability distributions. Acta Mathematica Academiae Scientiarum Hungarica 10, 193-215 (1959).

[218] Bittihn, P., Hörning, M. \& Luther, S. Negative curvature boundaries as wave emitting sites for the control of biological excitable media. Physical Review Letters 109, 118106 (2012).

[219] Takagi, S. et al. A physical approach to remove anatomical reentries: A bidomain study. Journal of Theoretical Biology 230, 489-97 (2004).

[220] Bittihn, P. Control of spiral wave activity in excitable media (University of Göttingen, 2009). Diploma Thesis.

[221] Kondratyev, A. A. et al. Virtual sources and sinks during extracellular field shocks in cardiac cell cultures: Effects of source-sink interactions between adjacent tissue boundaries. Circulation: Arrhythmia and Electrophysiology 5, 391-9 (2012).

[222] Stegun, I. \& Abramowitz, M. (eds.) Handbook of mathematical functions with formulas, graphs, and mathematical tables (National Bureau of Standards, Applied Mathematics Series 55, 1964). URL http://people.math.sfu.ca/ cbm/aands/. Tenth printing, December 1972.

[223] Fast, V. \& Kleber, A. Role of wavefront curvature in propagation of cardiac impulse. Cardiovascular Research 33, 258-71 (1997).

[224] Schröder-Schetelig, J. Experimentelle und theoretische Charakterisierung der Erregungsausbreitung im Kaninchenherzen anhand von Restitutionskurven (University of Göttingen, 2012). Diploma Thesis.

[225] Zhou, Y., Kassab, G. S. \& Molloi, S. On the design of the coronary arterial tree: A generalization of murray's law. Physics in Medicine and Biology 44, 2929-45 (1999).

[226] Kassab, G. S. Scaling laws of vascular trees: Of form and function. American Journal of Physiology: Heart and Circulatory Physiology 290, H894-903 (2006).

[227] Huo, Y. \& Kassab, G. S. A scaling law of vascular volume. Biophysical Journal 96, $347-53$ (2009).

[228] Santamore, W. P. \& Bove, A. A. Why are arteries the size they are? Journal of Applied Physiology 104, 1259 (2008).

[229] Hyatt, C., Mironov, S., Vetter, F., Zemlin, C. \& Pertsov, A. Optical action potential upstroke morphology reveals near-surface transmural propagation direction. Circulation Research 97, 277-84 (2005).

[230] Jenkins, M. Imaging the embryonic heart with optical coherence tomography (Case Western Reserve University, 2008). PhD Thesis.

[231] Mitrea, B. G., Caldwell, B. J. \& Pertsov, A. M. Imaging electrical excitation inside the myocardial wall. Biomedical Optics Express 2, 620-33 (2011). 
[232] Provost, J., Gambhir, A., Vest, J., Garan, H. \& Konofagou, E. E. A clinical feasibility study of atrial and ventricular electromechanical wave imaging. Heart Rhythm 10, 856-62 (2013).

[233] Veterkis, R., Grigaliunas, A. \& Mutskus, K. Comparative study of the space constant of electrotonic decay in the endocardium and epicardium of the rabbit right atrium. Biofizika 46, 310-4 (2001).

[234] Koster, R. et al. A randomized trial comparing monophasic and biphasic waveform shocks for external cardioversion of atrial fibrillation. American Heart Journal 147, 828.e1-7 (2004).

[235] Fast, V. G. \& Pertsov, A. M. Drift of vortex in the myocardium. Biofizika 35, 478-82 (1990).

[236] Nattel, S. Experimental evidence for proarrhythmic mechanisms of antiarrhythmic drugs. Cardiovascular Research 37, 567-77 (1998).

[237] Boyle, P. M., Deo, M., Plank, G. \& Vigmond, E. J. Purkinje-mediated effects in the response of quiescent ventricles to defibrillation shocks. Annals of Biomedical Engineering 38, 456-68 (2010).

[238] Cherry, E. M. \& Fenton, F. H. Effects of boundaries and geometry on the spatial distribution of action potential duration in cardiac tissue. Journal of Theoretical Biology 285, 164-76 (2011).

[239] Richter, C. Cardiomyocyten im Chaos - Makroskopische Untersuchungen kardialer Arrhytmien in-vitro unter dem Einfluss elektrischer Pulsfolgen und Parameteränderungen (University of Rostock, 2011). PhD Thesis.

[240] Fast, V., Rohr, S., Gillis, A. \& Kleber, A. Activation of cardiac tissue by extracellular electrical shocks: Formation of 'secondary sources' at intercellular clefts in monolayers of cultured myocytes. Circulation Research 82, 375-85 (1998).

[241] Isomura, A., Hörning, M., Agladze, K. \& Yoshikawa, K. Eliminating spiral waves pinned to an anatomical obstacle in cardiac myocytes by high-frequency stimuli. Physical Review E 78, 066216 (2008).

[242] Behrend, A., Bittihn, P. \& Luther, S. Predicting unpinning success rates for a pinned spiral in an excitable medium. Computing in Cardiology 345-8 (2010).

[243] Khait, V. D., Bernus, O., Mironov, S. F. \& Pertsov, A. M. Method for the threedimensional localization of intramyocardial excitation centers using optical imaging. Journal of Biomedical Optics 11, 34007 (2006).

[244] Clauset, A., Shalizi, C. R. \& Newman, M. E. J. Power-law distributions in empirical data. SIAM Review 51, 661-703 (2009).

[245] Virkar, Y. \& Clauset, A. Power-law distributions in binned empirical data. arXiv physics.data-an (2012). 33 pages, 9 figures, 3 tables, 2 appendices, $1208.3524 v 1$.

[246] Walton, R. D. et al. Extracting surface activation time from the optically recorded action potential in three-dimensional myocardium. Biophysical Journal 102, 30-8 (2012).

[247] Chaieb, L., Antal, A. \& Paulus, W. Transcranial alternating current stimulation in the low $\mathrm{kHz}$ range increases motor cortex excitability. Restorative Neurology and Neuroscience 29, 167-75 (2011). 
[248] Yang, H., Radons, G. \& Kantz, H. Covariant Lyapunov vectors from reconstructed dynamics: The geometry behind true and spurious Lyapunov exponents. Physical Review Letters 109, 244101 (2012).

[249] Fenton, F. \& Karma, A. Erratum: "Vortex dynamics in three-dimensional continuous myocardium with fiber rotation: Filament instability and fibrillation" [Chaos 8 , 20-47 (1998)]. Chaos 8, 879 (1998).

[250] Stroustrup, B. Principles and practice using C++ (Addison-Wesley, 2009).

[251] van Rossum, G. \& Drake, F. L. (eds.) Python reference manual (Python Labs, Virginia, USA). URL http://www.python.org.

[252] Numpy and Scipy. URL http://docs.scipy.org/doc// 


\section{Acknowledgments}

At this point, I would like to thank the various people and organizations which have supported me during the work for this thesis.

I would like to start by thanking my supervisor, Professor Dr. Stefan Luther, for giving me the opportunity to get involved into so many different and interesting projects with scientific problems from theoretical, experimental, basic and applied research, not all of which are covered by this thesis. Thank you for creating a research group with a unique and stimulating atmosphere, for giving me the freedom to take independent decisions on the scientific route to follow and for letting me present my work on a number of scientific conferences, which brought me into contact with the international cardiac and nonlinear dynamics community.

Moreover, I would like to express my gratitude to the other two members of my Thesis Committee, Professor Dr. Ulrich Parlitz and Professor Dr. Eberhard Bodenschatz, for their scientific and personal guidance not only during Thesis Committee meetings. I would also like to thank Professor Dr. Jörg Enderlein for his interest in my work and for agreeing to review this thesis.

Many thanks go to Professor Flavio H. Fenton for letting me visit his former place of work, Cornell University, and for many useful discussions on the theory and numerics of cardiac models, which significantly improved my understanding.

None of the work for this thesis would have been possible without the lab, IT and administrative infrastructure and the continuous support of the staff members maintaining it, first of all Dr. Denny Fliegner, Gerhard Nolte, Marion Kunze and Angela Meister. Thank you!

Science is team work. For all kinds of theoretical, numerical and technical problems, the members of the Biomedical Physics Group have been an invaluable source of inspiration and help. Special thanks go to my fellow "senior PhD student" (and now PhD) Amgad Squires, who introduced me to the technique of optical mapping and the corresponding data analysis concepts. Discussing scientific problems and developing new strategies was always productive and stimulating, even during phases of disappointment. Many thanks also to Daniel Hornung for joining the development of MediaSim and thereby contributing to the success it has become in the numerical work carried out in the Biomedical Physics Group. 
Furthermore, I would like to thank my collaborator Marcel Hörning for getting so interested in the theoretically-predicted boundary curvature effects that he agreed to immediately test its relevance in cell culture experiments.

I am grateful for the financial support by the International Max Planck Research School for the Physics of Biological and Complex systems, not only through a PhD stipend, but also by covering the costs for two conference participations and a one-month stay at Cornell University. I also acknowledge support by the Max Planck Society (through a PhD stipend), the DZHK (Deutsches Zentrum für Herz-Kreislauf-Forschung-German Centre for Cardiovascular Research) and by the BMBF (German Ministry of Education and Research). The research presented in this thesis has also received funding from the European Community's Seventh Framework Program FP7/2007-2013 under Grant Agreement No. HEALTH-F2-2009-241526, EUTrigTreat.

Last but not least, I am grateful to all my friends for the diversion outside work, particularly in form of making music, which was necessary to tackle scientific problems with a fresh view and regained energy. Many thanks to my family for their unconditional support in all possible situations, especially to my wife Katharina for her patience in times of increased work load and to my son Julius for bringing a whole new perspective to my life. 